WCH-412

Rev. 0

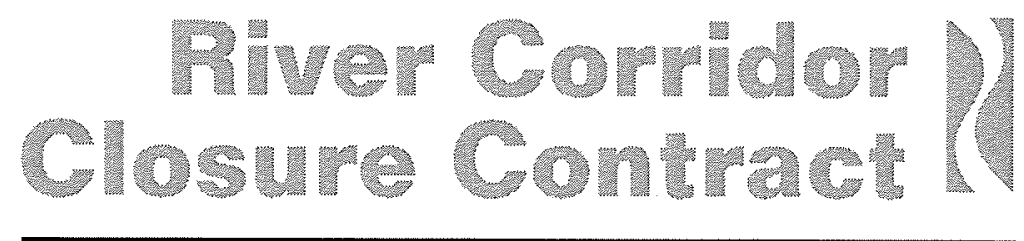

\title{
324 Building Baseline Radiological Characterization
}

\section{June 2010}

For Public Release

Washing con Closure Han?ord 
TRADEMARK DISCLAIMER

Reference herein to any specific commercial product, process, or service by trade name, trademark, manufacturer, or otherwise, does not necessarily constitute or imply its endorsement, recommendation, or favoring by the United States Government or any agency thereof or its contractors or subcontractors.

This report has been reproduced from the best available copy.

Printed in the United States of America 


\section{STANDARD APPROVAL PAGE}

Title:

324 Building Baseline Radiological Characterization

Author Name: R. J. Reeder, Radiological Control

J. C. Cooper, Radiological Control

Approval: $\quad$ G. A. Simiele, Radiological Control Manager
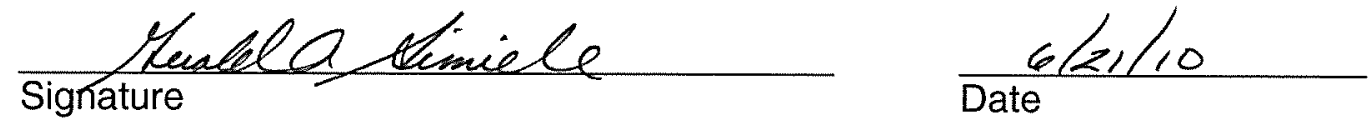

The approval signatures on this page indicate that this document has been authorized for information release to the public through appropriate channels. No other forms or signatures are required to document this information release. 
WCH-412

Rev. 0

\section{River Corridor Closure Contract}

\section{Building Baseline Radiological Characterization}

June 2010

Authors:

R. J. Reeder

J. C. Cooper

For Public Release 
. 


\section{EXECUTIVE SUMMARY}

This report documents the analysis of radiological data collected as part of the characterization study performed in 1998. The study was performed to create a baseline of the radiological conditions in the 324 Building.

A total of 85 technical [100 square centimeter $\left(\mathrm{cm}^{2}\right)$ ] smears were collected from the Room 147 hoods, the Shielded Materials Facility (SMF), and the Radiochemical Engineering Cells (REC). Exposure rate readings (window open and window closed) were taken at a distance of 2.5 centimeters $(\mathrm{cm})$ and $30 \mathrm{~cm}$ from the surface of each smear. Gross beta-gamma and alpha counts of each smear were also performed. The smear samples were analyzed by gamma energy analysis (GEA). Alpha energy analysis (AEA) and strontium-90 $\left({ }^{90} \mathrm{Sr}\right)$ analysis were also performed on selected smears. GEA results for one or more samples reported the presence of manganese-54 $\left({ }^{54} \mathrm{Mn}\right)$, cobalt-60 $\left({ }^{60} \mathrm{Co}\right)$, silver-108m $\left({ }^{108 \mathrm{~m}} \mathrm{Ag}\right)$, antimony-125 $\left({ }^{125} \mathrm{Sb}\right)$, cesium-134 $\left({ }^{134} \mathrm{Cs}\right)$, cesium-137 $\left({ }^{137} \mathrm{Cs}\right)$, europium-154 $\left({ }^{154} \mathrm{Eu}\right)$, europium-155 $\left({ }^{155} \mathrm{Eu}\right)$, and americium-241 $\left({ }^{241} \mathrm{Am}\right)$. AEA results reported the presence of plutonium-239/240 $\left({ }^{239 / 240} \mathrm{Pu}\right)$, plutonium-238 $\left({ }^{238} \mathrm{Pu}\right){ }^{241} \mathrm{Am}$, curium-243/244 $\left({ }^{243 / 244} \mathrm{Cm}\right)$, curium-242 $\left({ }^{242} \mathrm{Cm}\right)$, and americium-243 $\left({ }^{243} \mathrm{Am}\right)$.

Tables 5 through 9 present a summary by location of the estimated maximum removable and total contamination levels in the Room 147 hoods, the SMF, and the REC. The smear sample survey data and laboratory analytical results are presented in tabular form by sample in Appendix A. The Appendix A tables combine survey data documented in radiological survey reports found in Appendix $B$ and laboratory analytical results reported in the 324 Building Physical and Radiological Characterization Study (Berk, Hill, and Landsman 1998), supplemented by the laboratory analytical results found in Appendix C. 


\section{TABLE OF CONTENTS}

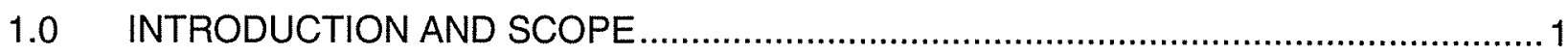

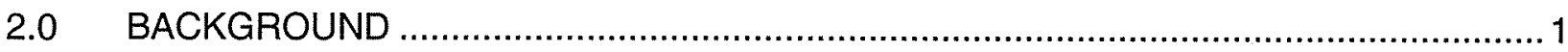

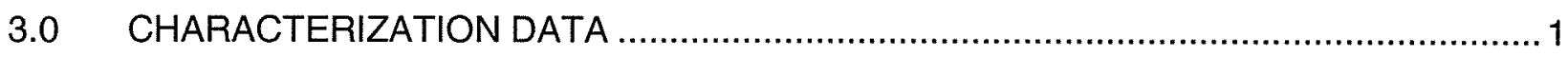

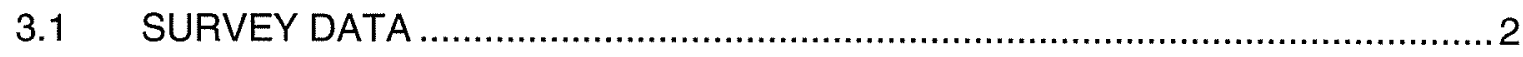

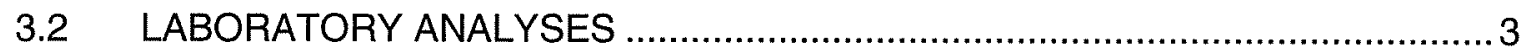

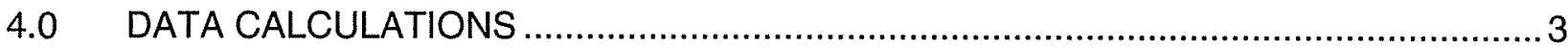

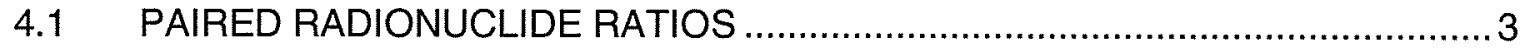

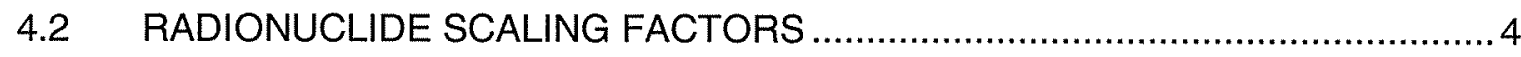

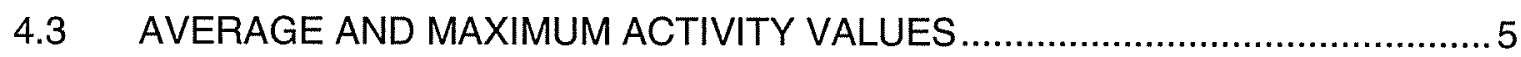

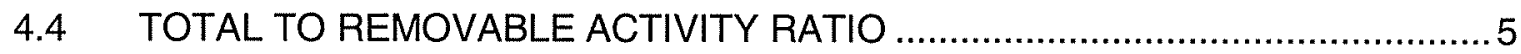

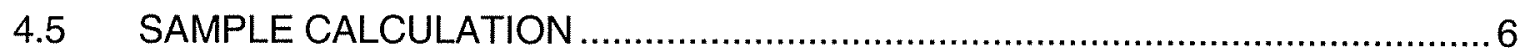

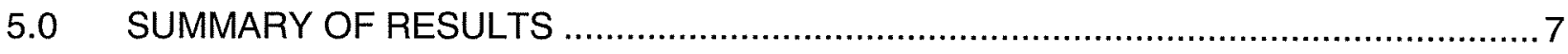




\section{APPENDICES}

A BASELINE RADIOLOGICAL CHARACTERIZATION DATA (TABULATED) .................

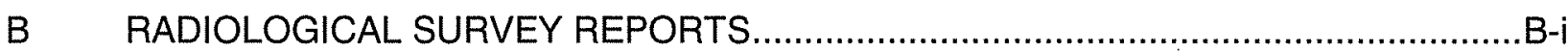

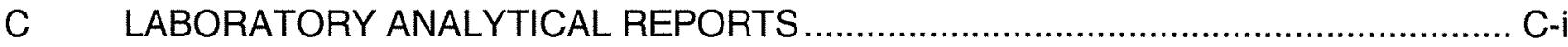

D TOTAL TO REMOVABLE ACTIVITY RATIO CALCULATION .................................. D-i

\section{TABLES}

Table 1. Summary of Smear Samples Collected and Analyses Performed .............................2

Table 2. Qualitative Summary of Measured Smear Results ................................................ 4

Table 3. Radionuclide Scaling Factors ........................................................................ 5

Table 4. C-Cell Sample 18a Measured and Calculated Activities............................................6

Table 5. Estimated Maximum Removable and Total Contamination - Room 147 Hoods.......... 7

Table 6. Estimated Maximum Removable Contamination Levels - SMF ............................... 8

Table 7. Estimated Maximum Total Contamination Levels - SMF ..................................... 9

Table 8. Estimated Maximum Removable Contamination Levels - REC ..............................10

Table 9. Estimated Maximum Total Contamination Levels - REC ..................................... 11 


\section{ACRONYMS AND ABBREVIATIONS}

\begin{tabular}{|c|c|}
\hline AEA & alpha energy analysis \\
\hline $\mathrm{Ag}$ & silver (e.g., ${ }^{108 \mathrm{~m}} \mathrm{Ag}$ ) \\
\hline $\mathrm{Am}$ & americium (e.g., $\left.{ }^{241} \mathrm{Am}\right)$ \\
\hline $\mathrm{Ba}$ & barium (e.g., $\left.{ }^{137 \mathrm{~m}} \mathrm{Ba}\right)$ \\
\hline $\mathrm{Cm}$ & curium (e.g. $\left.{ }^{243} \mathrm{Cm}\right)$ \\
\hline $\mathrm{cm}$ & centimeter \\
\hline $\mathrm{cm}^{2}$ & square centimeter \\
\hline Co & cobalt (e.g., $\left.{ }^{60} \mathrm{Co}\right)$ \\
\hline Cs & cesium (e.g., $\left.{ }^{137} \mathrm{Cs}\right)$ \\
\hline $\mathrm{CsCl}$ & cesium chloride \\
\hline dpm & disintegrations per minute \\
\hline Eu & europium (e.g., $\left.{ }^{155} \mathrm{Eu}\right)$ \\
\hline g & grams \\
\hline GEA & gamma energy analysis \\
\hline $\mathrm{HCl}$ & hydrochloric acid \\
\hline $\mathrm{HNO}_{3}$ & nitric acid \\
\hline M & molar \\
\hline $\mathrm{Mn}$ & manganese (e.g. $\left.{ }^{54} \mathrm{Mn}\right)$ \\
\hline $\mathrm{nCi}$ & nanocuries \\
\hline $\mathrm{Pu}$ & plutonium (e.g., $\left.{ }^{239} \mathrm{Pu}\right)$ \\
\hline REC & Radiochemical Engineering Cells \\
\hline $\mathrm{Se}$ & selenium (e.g., $\left.{ }^{79} \mathrm{Se}\right)$ \\
\hline $\mathrm{Sb}$ & antimony (e.g. $\left.{ }^{125} \mathrm{Sb}\right)$ \\
\hline $\mathrm{Sr}$ & strontium (e.g., $\left.{ }^{90} \mathrm{Sr}\right)$ \\
\hline SMF & Shielded Materials Facility \\
\hline TC & technetium (e.g., ${ }^{99} \mathrm{Tc}$ ) \\
\hline WCH & Washington Closure Hanford \\
\hline Y & yttrium (e.g. ${ }^{90} \mathrm{Y}$ ) \\
\hline
\end{tabular}




\section{METRIC CONVERSION CHART}

\begin{tabular}{|c|c|c|c|c|c|}
\hline \multicolumn{3}{|c|}{ Into Metric Units } & \multicolumn{3}{|c|}{ Out of Metric Units } \\
\hline If You Know & Multiply By & To Get & & Multiply By & To Get \\
\hline Length & & & Length & & \\
\hline inches & 25.4 & millimeters & millimeters & 0.039 & inches \\
\hline inches & 2.54 & centimeters & centimeters & 0.394 & inches \\
\hline feet & 0.305 & meters & meters & 3.281 & feet \\
\hline yards & 0.914 & meters & meters & 1.094 & yards \\
\hline miles & 1.609 & kilometers & kilometers & 0.621 & miles \\
\hline Area & & & Area & & \\
\hline sq. inches & 6.452 & sq. centimeters & sq. centimeters & 0.155 & sq. inches \\
\hline sq. feet & 0.093 & sq. meters & sq. meters & 10.76 & sq. feet \\
\hline sq. yards & 0.836 & sq. meters & sq. meters & 1.196 & sq. yards \\
\hline sq. miles & 2.6 & sq. kilometers & sq. kilometers & 0.4 & sq. miles \\
\hline acres & 0.405 & hectares & hectares & 2.47 & acres \\
\hline Mass (weight) & & & Mass (weight) & & \\
\hline ounces & 28.35 & grams & grams & 0.035 & ounces \\
\hline pounds & 0.454 & kilograms & kilograms & 2.205 & pounds \\
\hline ton & 0.907 & metric ton & metric ton & 1.102 & ton \\
\hline Volume & & & Volume & & \\
\hline teaspoons & 5 & milliliters & milliliters & 0.033 & $\begin{array}{l}\text { fluid } \\
\text { ounces }\end{array}$ \\
\hline tablespoons & 15 & milliliters & liters & 2.1 & pints \\
\hline fluid ounces & 30 & milliliters & liters & 1.057 & quarts \\
\hline cups & 0.24 & liters & liters & 0.264 & gallons \\
\hline pints & 0.47 & liters & cubic meters & 35.315 & cubic feet \\
\hline quarts & 0.95 & liters & cubic meters & 1.308 & $\begin{array}{l}\text { cubic } \\
\text { yards }\end{array}$ \\
\hline gallons & 3.8 & liters & & & \\
\hline cubic feet & 0.028 & cubic meters & & & \\
\hline cubic yards & 0.765 & cubic meters & & & \\
\hline Temperature & & & Temperature & & \\
\hline Fahrenheit & $\begin{array}{l}\text { subtract } 32 \text {, } \\
\text { then multiply } \\
\text { by } 5 / 9\end{array}$ & Celsius & Celsius & $\begin{array}{l}\text { multiply by } 9 / 5 \text {, } \\
\text { then add } 32\end{array}$ & Fahrenheit \\
\hline Radioactivity & & & Radioactivity & & \\
\hline picocuries & 37 & millibecquerel & millibecquerels & 0.027 & picocuries \\
\hline
\end{tabular}




\subsection{INTRODUCTION AND SCOPE}

This report documents the analysis of radiological data collected as part of the 1998 characterization study performed of the 324 Building. Radiological data were collected from the Room 147 hoods, the Shielded Materials Facility (SMF), and the Radiochemical Engineering Cells (REC). Data collected as part of the study from the SMF and the REC, specifically B-Cell and D-Cell, were analyzed and reported previously ${ }^{1}$, but are included here for completeness.

\subsection{BACKGROUND}

The characterization study performed in 1998 created a baseline of the radiological conditions in the 324 Building. The study, documented as Appendix D to the 324/327 Buildings Stabilization/ Deactivation Project Management Plan (Bechtel Hanford 1998) ${ }^{2}$, was conducted in two parts. First, radiological survey reports, chemical and radiological analytical reports, and historical records such as occurrence reports and program records were reviewed for data or conditions that may have affected the radiological status of the building. The information was assessed to determine which building areas required further characterization. The areas identified were the Room 147 hoods, the SMF, and the REC. Second, a sampling plan was developed to collect data from the building areas requiring further characterization. The sampling plan involved the collection of smear samples, performance of radiological field readings of the smears, and laboratory analysis of the smear samples.

\subsection{CHARACTERIZATION DATA}

A total of 85 technical [100 square centimeter $\left.\left(\mathrm{cm}^{2}\right)\right]$ smear samples were collected. The smear samples collected and laboratory analyses performed are summarized in Table 1. The smear sample data are presented in tabular form in Appendix A. The Appendix A tables combine survey data documented in radiological survey reports found in Appendix B and laboratory analytical results reported in the 324 Building Physical and Radiological Characterization Study (Berk, Hill, and Landsman 1998), supplemented by the laboratory analytical results found in Appendix C.

The 1998 characterization study data analyzed and reported previously are:

- SMF data: Radiological Characterization of the Shielded Material Facility (Mantooth 2003).

- REC B-Cell data: Data Analysis and Radiological Scaling Factor for the B-Cell Waste Stream, (Hill and Hobart 2000).

- REC D-Cell data: Radiological Characterization of the HLV Waste Treatment Skid in the 324 Facility (Hill 2001).

${ }^{2}$ The 1998 characterization study was removed with Revision 3 to the document. References to the 1998 characterization study are also found identifying it by the document number "HNF-3434." However, a search of archived records did not reveal a document by that number having ever been issued. 
Table 1. Summary of Smear Samples Collected and Analyses Performed

\begin{tabular}{|c|c|c|c|c|c|}
\hline \multirow{2}{*}{$\begin{array}{c}\text { Room or } \\
\text { Location }\end{array}$} & $\begin{array}{c}\text { Sample Location } \\
\text { Description }\end{array}$ & $\begin{array}{c}\text { Samples } \\
\text { Collected }\end{array}$ & \multicolumn{3}{|c|}{ Samples Analyzed } \\
\cline { 3 - 6 } & GEA & ${ }^{\text {(a) }} \mathbf{S r}^{(\mathbf{b})}$ & AEA $^{\text {(c) }}$ \\
\hline Room 147 & Hood \#1 & 2 & 2 & 1 & 2 \\
\cline { 2 - 6 } & Hood \#2 & 2 & 2 & 1 & 2 \\
\cline { 2 - 6 } & Hood \#3 & 2 & 2 & 1 & 2 \\
\hline \multirow{4}{*}{ SMF } & Airlock & 1 & 1 & 0 & 0 \\
\cline { 2 - 6 } & East Cell & 2 & 2 & 1 & 0 \\
\cline { 2 - 6 } & South Cell & 12 & 12 & 2 & 0 \\
\cline { 2 - 6 } & Compartment 1 & 4 & 4 & 1 & 0 \\
\cline { 2 - 6 } & Compartment 2 & 4 & 4 & 1 & 0 \\
\cline { 2 - 6 } & Compartment 3 & 4 & 4 & 1 & 0 \\
\cline { 2 - 6 } & Compartment 4 & 4 & 4 & 1 & 0 \\
\hline \multirow{4}{*}{ REC } & Airlock & 10 & 10 & 4 & 4 \\
\cline { 2 - 6 } & A-Cell & 6 & 6 & 2 & 2 \\
\cline { 2 - 6 } & B-Cell & 12 & 12 & 6 & 6 \\
\cline { 2 - 6 } & C-Cell & 10 & 10 & 5 & 6 \\
\cline { 2 - 6 } & D-Cell & 10 & 10 & 10 & 10 \\
\cline { 2 - 6 } & & & & & \\
\hline
\end{tabular}

(a) Gamma energy analysis

(b) Strontium-90 analysis

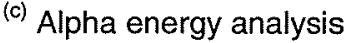

The data collected as part of the 1998 characterization study that were reported previously, but are also incorporated in Appendix A for completeness, include:

- SMF data: Mantooth (2003), Table 3-1, Summary of Analytical Results.

- REC B-Cell data: Hill and Hobart (2000), Table 7, Summary of Radiochemical Analysis of BCell Rack Metal Samples; and Table 9, Summary of Radiochemical Analysis of B-Cell Smears of Removable Contamination.

- REC D-Cell data: Hill (2001), Table 1, D-Cell Removable Contamination Characterization Data.

\subsection{SURVEY DATA}

Exposure rate readings (window open and window closed) were taken at a distance of 2.5 centimeters $(\mathrm{cm})$ and $30 \mathrm{~cm}$ from the surface of each smear. The window closed readings are reported in Appendix $A$ as gamma (penetrating radiation) exposure rates in units of milliroentgen per hour ( $\mathrm{mR} / \mathrm{hr})$. Beta correction factors were used to convert the window open exposure rate readings to absorbed (non-penetrating radiation) dose rates, which are reported in units of millirads per hour ( $\mathrm{mrad} / \mathrm{hr}$ ). The beta correction factors used are documented on the individual radiological survey reports in Appendix B.

Gross beta-gamma and alpha counts of each smear were also performed and, in some cases, performed multiple times (e.g, initial count plus 24-hour and/or 72-hour counts). Where more 
than one count was performed, the most recent values are reported in Appendix A (e.g., 24hour count results used in lieu of initial count results). The readings are reported in units of disintegrations per minute per $100 \mathrm{~cm}^{2}\left(\mathrm{dpm} / 100 \mathrm{~cm}^{2}\right)$.

\subsection{LABORATORY ANALYSES}

The smear samples were analyzed by gamma energy analysis (GEA). Alpha energy analysis (AEA) and strontium-90 $\left({ }^{90} \mathrm{Sr}\right)$ analysis were also performed on selected samples. GEA results for one or more samples reported the presence of manganese-54 $\left({ }^{54} \mathrm{Mn}\right)$, cobalt- $60\left({ }^{60} \mathrm{Co}\right)$, silver-108m ( $\left.{ }^{108 \mathrm{~m}} \mathrm{Ag}\right)$, antimony-125 $\left({ }^{125} \mathrm{Sb}\right)$, cesium-134 $\left({ }^{134} \mathrm{Cs}\right)$, cesium-137 $\left({ }^{137} \mathrm{Cs}\right)$, europium$154\left({ }^{154} \mathrm{Eu}\right)$, europium-155 $\left({ }^{155} \mathrm{Eu}\right)$, and americium-241 $\left({ }^{241} \mathrm{Am}\right)$. AEA results reported the presence of plutonium-239/240 $\left({ }^{239 / 240} \mathrm{Pu}\right)$, plutonium-238 $\left({ }^{238} \mathrm{Pu}\right){ }^{241} \mathrm{Am}$, curium-243/244 $\left({ }^{243 / 244} \mathrm{Cm}\right)$, curium-242 $\left({ }^{242} \mathrm{Cm}\right)$, and americium-243 $\left({ }^{243} \mathrm{Am}\right)$. The measured isotopic activities in Appendix $A$ are reported in units of microcuries per $100 \mathrm{~cm}^{2}\left(\mu \mathrm{Ci} / 100 \mathrm{~cm}^{2}\right)$.

Table 2 qualitatively summarizes the laboratory analytical results showing the radionuclides detected (i.e., activity reported above the detection limit) in one or more samples at each sample location.

\subsection{DATA CALCULATIONS}

The purpose of the baseline radiological characterization of the 324 Building was to obtain statistically reliable and defensible data to support waste classification in preparation for building dismantlement and demolition. Since direct measurement of all alpha-, beta-, and gammaemitting radionuclides in the waste was not economical or practical, indirect methods were used to complete the radiological profile. See Hill and Hobart (2000). The treatment of Appendix A data is described below. Neither the data nor the ratios and scaling factors used to complete the radiological profile were decay corrected.

\subsection{PAIRED RADIONUCLIDE RATIOS}

AEA results reported several radionuclides in pairs, i.e., ${ }^{239} \mathrm{Pu} /{ }^{240} \mathrm{Pu},{ }^{238} \mathrm{Pu} /{ }^{241} \mathrm{Am}$, and ${ }^{243} \mathrm{Cm} /{ }^{244} \mathrm{Cm}$. These results were separated into individual radionuclide activity values using assumptions applied in previous analyses of 324 Building radiological data. The assumptions and their sources are given below.

- $\quad{ }^{239} \mathrm{Pu} /{ }^{240} \mathrm{Pu}: 50.5 \%$ of total activity is ${ }^{239} \mathrm{Pu}$ and $49.5 \%$ is ${ }^{240} \mathrm{Pu}$. Source: Radiological Profile Methodology for Remote-Handled Transuranic Mixed Waste from 324 Facility B-Cell (Hill, et. al., 2000), pg. C-3. Assumption based on a ratio from commercial fuel sheared during the Nuclear Waste Vitrification Project.

- $\quad{ }^{238} \mathrm{Pu} /{ }^{241} \mathrm{Am}: 18.5 \%$ of total activity is ${ }^{238} \mathrm{Pu}$ and $81.5 \%$ is ${ }^{241} \mathrm{Am}$. Source: Hill, R. L., et. al. (2000), pgs. 22, 23. Assumption based relative isotopic concentrations on waste manifests of B-Cell dispersible material disposed of in 1996. 
- ${ }^{243} \mathrm{Cm} /{ }^{244} \mathrm{Cm}: 1.43 \%$ of total activity is ${ }^{243} \mathrm{Cm}$ and $98.6 \%$ is ${ }^{244} \mathrm{Cm}$. Source: Hill and Hobart (2000), pg. 34. Assumption based an estimated ${ }^{243} \mathrm{Cm}$ to ${ }^{244} \mathrm{Cm}$ ratio of $1: 70$ using process knowledge of the fuel that was used as a source of material added to B-Cell during its operational history.

Table 2. Qualitative Summary of Measured Smear Results

\begin{tabular}{|c|c|c|c|c|c|c|c|c|c|c|c|}
\hline \multirow{2}{*}{$\begin{array}{l}\text { Sample } \\
\text { Location }\end{array}$} & \multicolumn{6}{|c|}{ Gamma Energy Analysis } & \multirow{2}{*}{$\begin{array}{l}{ }^{90} \mathrm{Sr} \\
\text { Analysis }\end{array}$} & \multicolumn{4}{|c|}{ Alpha Energy Analysis } \\
\hline & ${ }^{54} \mathrm{Mn}$ & ${ }^{60} \mathrm{Co}$ & ${ }^{134} \mathrm{Cs}$ & ${ }^{137} \mathrm{Cs}$ & ${ }^{154} \mathrm{Eu}$ & ${ }^{241} \mathrm{Am}$ & & $\begin{array}{l}{ }^{239} \mathrm{Pu} / \\
{ }^{240} \mathrm{Pu}\end{array}$ & $\begin{array}{l}{ }^{238} \mathrm{Pu} / \\
{ }^{241} \mathrm{Am}\end{array}$ & $\begin{array}{l}{ }^{243} \mathrm{Cm} / \\
{ }^{24} \mathrm{Cm}\end{array}$ & ${ }^{242} \mathrm{Cm}$ \\
\hline \multicolumn{12}{|c|}{ Room 147 Hoods $^{a}$} \\
\hline Hood \#1 & & & & $\checkmark$ & & & $\checkmark$ & & & & \\
\hline Hood \#2 & & $\checkmark$ & & $\checkmark$ & & & $\checkmark$ & & $\checkmark$ & & \\
\hline Hood \#3 & $\checkmark$ & $\checkmark$ & & $\checkmark$ & & & $\checkmark$ & & & & \\
\hline \multicolumn{12}{|c|}{ SMF $^{a}$} \\
\hline Airlock & & & & & & & --- & -.. & -.. & --. & --- \\
\hline East Cell & & $\checkmark$ & $\checkmark$ & $\checkmark$ & & & $\checkmark$ & $\cdots$ & $-\cdots$ & $-\cdots$ & $\cdots$ \\
\hline South Cell & $\checkmark$ & $\checkmark$ & $\checkmark$ & $\checkmark$ & & & $\checkmark$ & $-\cdots$ & 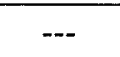 & $\cdots$ & $\cdots$ \\
\hline Comp. \#1 & & & & $\checkmark$ & & & $\checkmark$ & --- & $-\cdots$ & $\cdots$ & $-\cdots$ \\
\hline Comp. \#2 & & $\checkmark$ & $\checkmark$ & $\checkmark$ & & & $\checkmark$ & $-\cdots$ & $\cdots$ & $\overline{---}$ & $-\cdots$ \\
\hline Comp. \#3 & $\checkmark$ & $\checkmark$ & $\checkmark$ & $\checkmark$ & & & $\checkmark$ & --- & -- & $-\cdots$ & $\cdots$ \\
\hline Comp. \#4 & $\checkmark$ & $\checkmark$ & $\checkmark$ & $\checkmark$ & & & $\checkmark$ & --- & $-\cdots$ & --- & -- \\
\hline \multicolumn{12}{|c|}{ REC $^{a}$} \\
\hline Airlock $^{b}$ & & $\checkmark$ & $\checkmark$ & $\checkmark$ & $\checkmark$ & & $\checkmark$ & $\checkmark$ & $\checkmark$ & $\checkmark$ & $\checkmark$ \\
\hline A-Cell & & $\checkmark$ & & $\checkmark$ & & & $\checkmark$ & 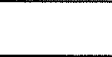 & $\checkmark$ & $\checkmark$ & \\
\hline B-Cell & & $\checkmark$ & $\checkmark$ & $\checkmark$ & $\checkmark$ & $\checkmark$ & $\checkmark$ & $\checkmark$ & $\checkmark$ & $\checkmark$ & \\
\hline C-Cell ${ }^{c}$ & & $\checkmark$ & $\checkmark$ & $\checkmark$ & $\checkmark$ & $\checkmark$ & $\checkmark$ & $\checkmark$ & $\checkmark$ & $\checkmark$ & \\
\hline D-Cell ${ }^{d}$ & & $\checkmark$ & $\checkmark$ & $\checkmark$ & $\checkmark$ & $\checkmark$ & $\checkmark$ & $\checkmark$ & $\checkmark$ & $\checkmark$ & $\checkmark$ \\
\hline
\end{tabular}

${ }^{a}$ Check mark indicates radionuclide detected, i.e., reported activity above detection limit; blank cell indicates reported activity below detection limit; and dashed line indicates no analyses performed.

${ }^{\mathrm{b}}$ Detectable levels of ${ }^{243} \mathrm{Am}$ identified in three samples.

${ }^{c}$ Detectable levels of ${ }^{155} \mathrm{Eu}$ also identified in five samples.

${ }^{d}$ Detectable levels of ${ }^{108 \mathrm{~m}} \mathrm{Ag}$ also identified in one sample.

\subsection{RADIONUCLIDE SCALING FACTORS}

Isotopic activity values for ${ }^{241} \mathrm{Pu},{ }^{242} \mathrm{Pu}$, selenium-79 $\left({ }^{79} \mathrm{Se}\right)$, technetium-99 $\left({ }^{99} \mathrm{Tc}\right)$, and ${ }^{90} \mathrm{Sr}$ were calculated based on previously determined scaling factors used in Hill and Hobart (2000). The scaling factors and their surrogate radionuclides are given in Table 3 . The basis for inclusion of the radionuclides in Table 3 is given in Hill and Hobart (2000).

The individual bases for the radionuclide scaling factors are as follows:

- ${ }^{241} \mathrm{Pu},{ }^{242} \mathrm{Pu}$ : The isotopic composition of the light water reactor fuel assemblies used in the Nuclear Waste Vitrification Project source term was assumed to bound the work 
done throughout the work life-time of B-Cell and was therefore used as the plutonium composition for waste classification purposes (Hill and Hobart 2000, pg. 41).

- ${ }^{79} \mathrm{Se},{ }^{99} \mathrm{Tc}$ : A ratioing method using ORIGEN data was used for these mobile radionuclides that could not be entirely dismissed in earlier B-Cell waste characterization efforts (Hill and Hobart 2000, pg. 40).

- ${ }^{90} \mathrm{Sr}$ : The relationship between ${ }^{90} \mathrm{Sr}$ and ${ }^{137} \mathrm{Cs}$ was established based on the analysis of smears of removable contamination collected in B-Cell obtained under controlled conditions (Hill and Hobart 2000, pg. 52). This relationship does not hold for the SMF where cesium chloride (CsCl) was processed and encapsulated (Mantooth 2003, pg. 1). Accordingly, this scaling factor was not applied to SMF data.

Table 3. Radionuclide Scaling Factors

\begin{tabular}{|c|c|c|}
\hline Radionuclide & $\begin{array}{c}\text { Scaling } \\
\text { Factor }\end{array}$ & $\begin{array}{c}\text { Surrogate } \\
\text { Radionuclide }\end{array}$ \\
\hline${ }^{241} \mathrm{Pu}$ & $2.47 \mathrm{E}+01$ & ${ }^{239 / 240} \mathrm{Pu}$ \\
\hline${ }^{242} \mathrm{Pu}$ & $8.28 \mathrm{E}-04$ & ${ }^{239 / 240} \mathrm{Pu}$ \\
\hline${ }^{79} \mathrm{Se}$ & $2.36 \mathrm{E}-04$ & ${ }^{241} \mathrm{Am}$ \\
\hline${ }^{99} \mathrm{Tc}$ & $7.83 \mathrm{E}-03$ & ${ }^{241} \mathrm{Am}$ \\
\hline${ }^{90} \mathrm{Sr}$ & $6.00 \mathrm{E}-01$ & ${ }^{137} \mathrm{Cs}$ \\
\hline
\end{tabular}

\subsection{AVERAGE AND MAXIMUM ACTIVITY VALUES}

Average and maximum removable activity values were calculated by sample location for each radionuclide. Samples with reported activity below the detection limit, i.e., less-than values, were not used in the statistical calculations. Average and maximum total activity values were calculated by multiplying the removable activity values by a factor of 30 . This factor is based on the total to removable activity ratio developed as described below.

\subsection{TOTAL TO REMOVABLE ACTIVITY RATIO}

The total to removable activity ratio of 30:1 was developed using data reported in Hill and Hobart (2000). Fixed radioactive contamination on the surface of metal coupon samples collected from B-Cell process equipment was removed by etching the surface of the coupon with diluted Aqua Regia [ 3 molar $(\mathrm{M})$ nitric acid $\left(\mathrm{HNO}_{3}\right)$ and $3 \mathrm{M}$ hydrochloric $(\mathrm{HCl})$ mixture]. The resulting solutions were analyzed by GEA, AEA, beta counting $\left({ }^{90} \mathrm{Sr}\right)$, proportional counting (gross alpha), inductively coupled plasma mass spectrometry $\left({ }^{243} \mathrm{Cm},{ }^{244} \mathrm{Cm}\right.$ ), and laser kinetic phosphorescence (total uranium). Results above detection levels were reported for ${ }^{90} \mathrm{Sr},{ }^{125} \mathrm{Sb}$, ${ }^{137} \mathrm{Cs},{ }^{154} \mathrm{Eu},{ }^{238} \mathrm{Pu},{ }^{239 / 240} \mathrm{Pu},{ }^{241} \mathrm{Am},{ }^{243} \mathrm{Cm}$, and ${ }^{244} \mathrm{Cm}$.

Hill and Hobart (2000), Table 7, reported the results in units of nanocuries per gram (nCi/g). The results were converted from $\mathrm{nCi} / \mathrm{g}$ to $\mathrm{nCi} / 100 \mathrm{~cm}^{2}$ using the effective etched surface area $\left(\mathrm{cm}^{2}\right)$, and the corresponding etched area mass $(\mathrm{g})$ of each sample. Now in compatible units, the fixed contamination results were compared to the analytical results of the removable contamination smear samples collected from B-Cell. The removable contamination results are 
reported in Hill and Hobart (2000), Table 9, in units of $\mathrm{nCi} / 100 \mathrm{~cm}^{2}$. The total to removable activity ratio calculations are found in Appendix $D$.

\subsection{SAMPLE CALCULATION}

The manipulation of the laboratory analytical results to arrive at the calculated activities reported in Appendix A is illustrated in Table 4. Sample 18a, collected from REC C-Cell, is used as the example. Three types of analyses were performed on the sample, from which 8 results were reported above the corresponding detection limit. The measured activities were used in conjunction with the ratios and scaling factors described in Sections 4.1 and 4.2 to complete the radiological profile of the sample.

The three columns on the left-hand side of Table 4 show the types of analyses performed on the smear sample, the radionuclides detected, and their measured activities. The three columns on the right-hand side of the table list the inferred radionuclides, the ratio or scaling factor equation used to calculate their activities, and their calculated activities.

For the sample used in the example, ${ }^{90} \mathrm{Sr}$ analysis was not performed. Since directly measured ${ }^{90} \mathrm{Sr}$ data were not available, an activity for ${ }^{90} \mathrm{Sr}$ was calculated using the scaling factor based on ${ }^{137} \mathrm{Cs}$. GEA analysis was performed and reported a measured activity for ${ }^{241} \mathrm{Am}$. Since directly measured data for ${ }^{241} \mathrm{Am}$ were available, the ratio based on the measured ${ }^{238} \mathrm{Pu} /{ }^{241} \mathrm{Am}$ activity was not used.

Table 4. C-Cell Sample 18a Measured and Calculated Activities

\begin{tabular}{|c|c|c|c|c|c|}
\hline Analysis & $\begin{array}{c}\text { Surrogate } \\
\text { Radionuclide }\end{array}$ & $\begin{array}{c}\text { Measured } \\
\text { Activity } \\
\left(\mu \mathrm{Ci} / 100 \mathrm{~cm}^{2}\right)\end{array}$ & $\begin{array}{c}\text { Inferred } \\
\text { Radionuclide }\end{array}$ & $\begin{array}{c}\text { Ratio/Scaling Factor } \\
\text { Equation }\end{array}$ & $\begin{array}{c}\text { Calculated } \\
\text { Activity } \\
\left(\mu \mathrm{Ci} / 100 \mathrm{~cm}^{2}\right)\end{array}$ \\
\hline \multirow[t]{5}{*}{ GEA } & ${ }^{134} \mathrm{Cs}$ & $4.09 \mathrm{E}-03$ & $\cdots$ & -- & --. \\
\hline & ${ }^{137} \mathrm{Cs}$ & $1.10 E+00$ & ${ }^{90} \mathrm{Sr}$ & $6.00 \mathrm{E}-01 \times{ }^{137} \mathrm{Cs}$ & 6.60E-01 \\
\hline & ${ }^{154} \mathrm{Eu}$ & $2.25 \mathrm{E}-03$ & $\cdots$ & $-\cdots$ & --- \\
\hline & \multirow[t]{2}{*}{${ }^{241} \mathrm{Am}$} & $1.51 \mathrm{E}-03$ & ${ }^{79} \mathrm{Se}$ & $2.36 \mathrm{E}-04 \mathrm{x}^{241} \mathrm{Am}$ & $3.56 \mathrm{E}-07$ \\
\hline & & & ${ }^{99} \mathrm{Tc}$ & $7.83 \mathrm{E}-03 \mathrm{x}^{241} \mathrm{Am}$ & 1.18E-05 \\
\hline \multirow[t]{7}{*}{ AEA } & ${ }^{238} \mathrm{Pu} /{ }^{241} \mathrm{Am}$ & 3.27E-03 & ${ }^{238} \mathrm{Pu}$ & $1.85 \mathrm{E}-01 \mathrm{x}^{238} \mathrm{Pu} /{ }^{241} \mathrm{Am}$ & $6.05 E-04$ \\
\hline & \multirow[t]{4}{*}{${ }^{239} \mathrm{Pu} /{ }^{240} \mathrm{Pu}$} & \multirow[t]{4}{*}{$5.69 \mathrm{E}-04$} & ${ }^{239} \mathrm{Pu}$ & $5.05 \mathrm{E}-01 \times{ }^{239} \mathrm{Pu} /{ }^{240} \mathrm{Pu}$ & 2.87E-04 \\
\hline & & & ${ }^{240} \mathrm{Pu}$ & 4.95E-01 $x^{239} \mathrm{Pu} /{ }^{240} \mathrm{Pu}$ & $2.82 E-04$ \\
\hline & & & ${ }^{241} \mathrm{Pu}$ & $2.47 E+01 \times{ }^{239} \mathrm{Pu} /{ }^{240} \mathrm{Pu}$ & $1.41 \mathrm{E}-02$ \\
\hline & & & ${ }^{242} \mathrm{Pu}$ & $8.28 \mathrm{E}-04 \mathrm{x}^{239} \mathrm{Pu} /{ }^{240} \mathrm{Pu}$ & $4.71 E-07$ \\
\hline & \multirow[t]{2}{*}{${ }^{243} \mathrm{Cm} /{ }^{244} \mathrm{Cm}$} & \multirow[t]{2}{*}{$1.15 \mathrm{E}-02$} & ${ }^{243} \mathrm{Cm}$ & 1.43E-02 $\mathrm{x}^{243} \mathrm{Cm} /{ }^{244} \mathrm{Cm}$ & $1.64 \mathrm{E}-04$ \\
\hline & & & ${ }^{244} \mathrm{Cm}$ & $9.86 \mathrm{E}-01 \mathrm{x}^{243} \mathrm{Cm} /{ }^{244} \mathrm{Cm}$ & 1.13E-02 \\
\hline \multirow{2}{*}{$\begin{array}{l}\text { Gross } \\
\text { Alpha }\end{array}$} & \multirow[t]{2}{*}{ Alpha } & \multirow[t]{2}{*}{$1.53 E-02$} & Beta/Gamma & ${ }^{90} \mathrm{Sr}+{ }^{134} \mathrm{Cs}+{ }^{137} \mathrm{Cs}+{ }^{154} \mathrm{Eu}$ & $1.77 \mathrm{E}+00$ \\
\hline & & & Total Activity & Alpha + Beta/Gamma & $1.78 E+00$ \\
\hline
\end{tabular}




\subsection{SUMMARY OF RESULTS}

Tables 5 through 9 present a summary by location of the estimated maximum removable and total contamination levels in the Room 147 hoods, the SMF, and the REC. The results by individual sample are found in Appendix A.

Table 5. Estimated Maximum Removable and Total Contamination - Room 147 Hoods

\begin{tabular}{|c|c|c|c|c|c|c|}
\hline \multirow{2}{*}{ Radionuclide } & \multicolumn{3}{|c|}{ Removable $\left(\mu \mathrm{Ci} / 100 \mathrm{~cm}^{2}\right)$} & \multicolumn{3}{|c|}{ Total $\left(\mu \mathrm{Ci} / 100 \mathrm{~cm}^{2}\right)$} \\
\hline & Hood \#1 & Hood \#2 & Hood \#3 & Hood \#1 & Hood \#2 & Hood \#3 \\
\hline${ }^{54} \mathrm{Mn}$ & ND & ND & $1.05 \mathrm{E}-05$ & ND & ND & $3.15 \mathrm{E}-04$ \\
\hline${ }^{60} \mathrm{Co}$ & ND & $9.11 E-04$ & 5.97E-04 & ND & 2.73E-02 & $1.79 \mathrm{E}-02$ \\
\hline${ }^{79} \mathrm{Se}$ & $\cdots$ & 1.79E-09 & $\cdots$ & --- & 5.37E-08 & $\cdots$ \\
\hline${ }^{90} \mathrm{Sr}^{\mathrm{a}}$ & 8.46E-03 & 1.16E-02 & $1.44 \mathrm{E}-03$ & 2.54E-01 & $3.47 \mathrm{E}-01$ & 4.32E-02 \\
\hline${ }^{99} \mathrm{Tc}$ & --- & $5.94 E-08$ & --- & -- & $1.78 \mathrm{E}-06$ & -- \\
\hline${ }^{108 m} \mathrm{Ag}$ & $-\cdots$ & --- & $\cdots$ & $-\cdots$ & -- & $-\cdots$ \\
\hline${ }^{125} \mathrm{Sb}$ & ND & ND & ND & ND & ND & ND \\
\hline${ }^{134} \mathrm{Cs}$ & ND & ND & ND & ND & ND & ND \\
\hline${ }^{137} \mathrm{Cs}^{\mathrm{b}}$ & $1.41 E-02$ & $1.93 \mathrm{E}-02$ & 3.61E-03 & $4.23 E-01$ & 5.79E-01 & $1.08 \mathrm{E}-01$ \\
\hline${ }^{154} \mathrm{Eu}$ & ND & ND & ND & ND & ND & ND \\
\hline${ }^{155} \mathrm{Eu}$ & ND & ND & ND & ND & ND & ND \\
\hline${ }^{238} \mathrm{Pu}$ & --- & 1.72E-06 & $-\cdots$ & --- & 5.17E-05 & -..- \\
\hline${ }^{239} \mathrm{Pu}$ & $\cdots$ & $\cdots$ & -- & --- & -- & --- \\
\hline${ }^{240} \mathrm{Pu}$ & $-\cdots$ & --- & -- & $-\cdots$ & $\cdots$ & --- \\
\hline${ }^{241} \mathrm{Pu}$ & $-\cdots$ & $\cdots$ & $\cdots$ & --- & $\cdots$ & --- \\
\hline${ }^{242} \mathrm{Pu}$ & $\cdots$ & -- & --- & $\cdots$ & $\cdots$ & --- \\
\hline${ }^{241} \mathrm{Am}$ & -- & 7.59E-06 & -- & -- & $2.28 \mathrm{E}-04$ & -- \\
\hline${ }^{242} \mathrm{Cm}$ & ND & ND & ND & ND & ND & ND \\
\hline${ }^{243} \mathrm{Cm}$ & --- & $\cdots$ & $\cdots$ & -- & --- & --- \\
\hline${ }^{244} \mathrm{Cm}$ & $\cdots$ & $\cdots$ & $\cdots$ & -- & $\cdots$ & $\cdots$ \\
\hline Alpha & ND & $1.49 \mathrm{E}-05$ & ND & ND & 4.47E-04 & ND \\
\hline Beta-Gamma & 2.26E-02 & 3.10E-02 & 4.45E-03 & $6.77 \mathrm{E}-01$ & 9.31E-01 & 1.33E-01 \\
\hline Total Activity $^{c}$ & 2.26E-02 & 3.11E-02 & 4.45E-03 & $6.77 \mathrm{E}-01$ & $9.32 E-01$ & 1.33E-01 \\
\hline
\end{tabular}

$\mathrm{ND}=$ not detected

--- $=$ not reported

${ }^{a}$ assumed to include yttrium- $90\left({ }^{90} \mathrm{Y}\right)$ in secular equilibrium

${ }^{b}$ assumed to include barium-137m $\left({ }^{137 m} \mathrm{Ba}\right)$ in secular equilibrium

' alpha + beta-gamma activity 
Table 6. Estimated Maximum Removable Contamination Levels - SMF

\begin{tabular}{|c|c|c|c|c|c|c|c|}
\hline \multirow[b]{2}{*}{ Radionuclide } & \multicolumn{7}{|c|}{ Removable Contamination $\left(\mu \mathrm{Ci} / 100 \mathrm{~cm}^{2}\right)$} \\
\hline & Airlock & East Cell & $\begin{array}{l}\text { South } \\
\text { Cell }\end{array}$ & $\begin{array}{c}\text { Comp. } \\
\# 1\end{array}$ & $\begin{array}{c}\text { Comp. } \\
\# 2\end{array}$ & $\begin{array}{c}\text { Comp. } \\
\# 3\end{array}$ & $\begin{array}{c}\text { Comp. } \\
\text { \#4 }\end{array}$ \\
\hline${ }^{54} \mathrm{Mn}$ & ND & ND & 1.93E-01 & ND & ND & $3.59 \mathrm{E}-04$ & $9.22 E-04$ \\
\hline${ }^{60} \mathrm{Co}$ & ND & $1.81 E-05$ & $1.34 \mathrm{E}+01$ & ND & $1.49 \mathrm{E}-03$ & $9.58 E-03$ & $3.21 E-02$ \\
\hline${ }^{79} \mathrm{Se}$ & --- & --- & -.- & --- & $\ldots$ & --- & $-\cdots$ \\
\hline${ }^{90} \mathrm{Sr}^{\mathrm{a}}$ & $-\cdots$ & $4.59 \mathrm{E}-02$ & $8.34 \mathrm{E}+00$ & $4.81 E+00$ & 1.30E-03 & 1.09E-03 & 4.90E-03 \\
\hline${ }^{99} \mathrm{Tc}$ & -.. & -.- & $-\ldots$ & --- & $\ldots$ & --- & $\ldots$ \\
\hline${ }^{108 m} \mathrm{Ag}$ & --- & -.. & $-\cdots$ & --- & $-\cdots$ & --- & --- \\
\hline${ }^{125} \mathrm{Sb}$ & ND & ND & ND & ND & ND & ND & $2.47 E-03$ \\
\hline${ }^{134} \mathrm{Cs}$ & ND & 1.53E-05 & $2.21 E+00$ & ND & $2.11 E-04$ & 5.63E-04 & $3.48 E-03$ \\
\hline${ }^{137} \mathrm{Cs}^{\mathrm{b}}$ & ND & 1.53E-03 & $2.76 \mathrm{E}+02$ & $1.04 E+03$ & 1.49E-01 & $2.08 E-01$ & $2.19 \mathrm{E}-01$ \\
\hline${ }^{154} \mathrm{Eu}$ & ND & ND & ND & ND & ND & ND & ND \\
\hline${ }^{155} \mathrm{Eu}$ & ND & ND & ND & ND & ND & ND & ND \\
\hline${ }^{238} \mathrm{Pu}$ & 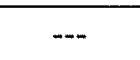 & $\cdots$ & $-\cdots$ & $-\cdots$ & --- & $\cdots$ & --- \\
\hline${ }^{239} \mathrm{Pu}$ & --- & -- & 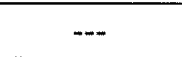 & -- & $-\cdots$ & $\cdots$ & --- \\
\hline${ }^{240} \mathrm{Pu}$ & --- & $-\cdots$ & $\ldots$ & -- & $\ldots$ & --- & --- \\
\hline${ }^{241} \mathrm{Pu}$ & $\cdots$ & $-\cdots$ & --- & $\cdots$ & -.. & --- & -.. \\
\hline${ }^{242} \mathrm{Pu}$ & $-\cdots$ & --- & --- & $-\cdots$ & --- & $\ldots$ & --- \\
\hline${ }^{241} \mathrm{Am}$ & --- & --- & --- & -- & $\cdots$ & -.. & $-\cdots$ \\
\hline${ }^{242} \mathrm{Cm}$ & --- & --- & --- & $-\cdots$ & 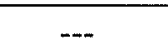 & $-\cdots$ & $-\cdots$ \\
\hline${ }^{243} \mathrm{Cm}$ & -- & $\ldots$ & --- & ... & -.- & --- & --- \\
\hline${ }^{244} \mathrm{Cm}$ & $-\cdots$ & $-\cdots$ & $\cdots$ & --- & $\cdots$ & -- & $-\cdots$ \\
\hline Alpha & ND & $1.03 E-05$ & 4.81E-03 & $4.06 \mathrm{E}-03$ & ND & ND & ND \\
\hline Beta-Gamma & ND & 4.72E-02 & $2.88 E+02$ & $1.04 E+03$ & $1.50 \mathrm{E}-01$ & $2.16 \mathrm{E}-01$ & $2.25 E-01$ \\
\hline Total Activity $^{c}$ & ND & 4.73E-02 & $2.88 E+02$ & $1.04 E+03$ & $1.50 \mathrm{E}-01$ & $2.16 \mathrm{E}-01$ & $2.25 E-01$ \\
\hline
\end{tabular}

$\mathrm{ND}=$ not detected

-.. $=$ not reported

${ }^{a}$ assumed to include ${ }^{90} \mathrm{Y}$ in secular equilibrium

${ }^{b}$ assumed to include ${ }^{137 m} \mathrm{Ba}$ in secular equilibrium

${ }^{\mathrm{C}}$ alpha + beta-gamma activity 
Table 7. Estimated Maximum Total Contamination Levels - SMF

\begin{tabular}{|c|c|c|c|c|c|c|c|}
\hline \multirow[b]{2}{*}{ Radionuclide } & \multicolumn{7}{|c|}{ Total Contamination $\left(\mu \mathrm{Ci} / 100 \mathrm{~cm}^{2}\right)$} \\
\hline & Airlock & East Cell & $\begin{array}{c}\text { South } \\
\text { Cell }\end{array}$ & $\begin{array}{c}\text { Comp. } \\
\# 1\end{array}$ & $\begin{array}{c}\text { Comp. } \\
\# 2\end{array}$ & $\begin{array}{c}\text { Comp. } \\
\# 3\end{array}$ & $\underset{\# 4}{\text { Comp. }}$ \\
\hline${ }^{54} \mathrm{Mn}$ & ND & ND & $5.79 \mathrm{E}+00$ & ND & ND & 1.08E-02 & 2.77E-02 \\
\hline${ }^{60} \mathrm{Co}$ & ND & $5.43 E-04$ & $4.02 \mathrm{E}+02$ & ND & 4.47E-02 & 2.87E-01 & $9.63 \mathrm{E}-01$ \\
\hline${ }^{79} \mathrm{Se}$ & $\cdots$ & -- & -- & -- & --- & $\cdots$ & --- \\
\hline${ }^{90} \mathrm{Sr}^{\mathrm{a}}$ & -- & $1.38 \mathrm{E}+00$ & $2.50 E+02$ & $1.44 E+02$ & $3.90 \mathrm{E}-02$ & 3.27E-02 & 1.47E-01 \\
\hline${ }^{99} \mathrm{Tc}$ & $-\cdots$ & --- & -- & $\ldots$ & --- & --- & $-\ldots$ \\
\hline${ }^{108 \mathrm{~m}} \mathrm{Ag}$ & $-\cdots$ & --- & --- & $\cdots$ & -- & --- & -- \\
\hline${ }^{125} \mathrm{Sb}$ & ND & ND & ND & ND & ND & ND & $7.41 E-02$ \\
\hline${ }^{134} \mathrm{Cs}$ & ND & $4.59 \mathrm{E}-04$ & $6.63 E+01$ & ND & 6.33E-03 & $1.69 \mathrm{E}-02$ & $1.04 \mathrm{E}-01$ \\
\hline${ }^{137} \mathrm{Cs}^{\mathrm{b}}$ & ND & 4.59E-02 & $8.28 \mathrm{E}+03$ & $3.12 E+04$ & $4.47 E+00$ & $6.24 \mathrm{E}+00$ & $6.57 E+00$ \\
\hline${ }^{154} \mathrm{Eu}$ & ND & ND & ND & ND & ND & ND & ND \\
\hline${ }^{155} \mathrm{Eu}$ & ND & ND & ND & ND & ND & ND & ND \\
\hline${ }^{238} \mathrm{Pu}$ & $-\cdots$ & --- & $\cdots$ & $-\cdots$ & -- & -- & $\cdots$ \\
\hline${ }^{239} \mathrm{Pu}$ & $-\cdots$ & --- & -- & $\cdots$ & --- & $-\ldots$ & $-\ldots$ \\
\hline${ }^{240} \mathrm{Pu}$ & --- & --- & $-\cdots$ & $\cdots$ & $-\cdots$ & $-\ldots$ & $-\cdots$ \\
\hline${ }^{241} \mathrm{Pu}$ & --- & -- & --- & $\cdots$ & $\ldots$ & $-\cdots$ & --- \\
\hline${ }^{242} \mathrm{Pu}$ & --- & -- & -- & --- & $\cdots$ & $\cdots$ & $-\cdots$ \\
\hline${ }^{241} \mathrm{Am}$ & -- & $\cdots$ & --- & $-\cdots$ & $-\cdots$ & $\cdots$ & $\cdots$ \\
\hline${ }^{242} \mathrm{Cm}$ & $-\cdots$ & -- & --- & $\cdots$ & --- & $-\cdots$ & $-\cdots$ \\
\hline${ }^{243} \mathrm{Cm}$ & $\cdots$ & --- & --- & -- & --- & $\cdots$ & 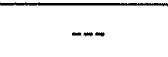 \\
\hline${ }^{244} \mathrm{Cm}$ & --- & $\ldots$ & -- & -- & $-\cdots$ & $\ldots$ & $-\cdots$ \\
\hline Alpha & ND & 3.09E-04 & 1.44E-01 & $1.22 \mathrm{E}-01$ & ND & ND & ND \\
\hline Beta-Gamma & ND & $1.42 \mathrm{E}+00$ & $8.64 E+03$ & $3.12 E+04$ & $4.51 E+00$ & $6.47 E+00$ & $6.76 E+00$ \\
\hline Total Activity $^{c}$ & ND & $1.42 \mathrm{E}+00$ & $8.64 E+03$ & $3.12 E+04$ & $4.51 E+00$ & $6.47 \mathrm{E}+00$ & $6.76 E+00$ \\
\hline
\end{tabular}

ND $=$ not detected

$---=$ not reported

${ }^{\mathrm{a}}$ assumed to include ${ }^{90} \mathrm{Y}$ in secular equilibrium

${ }^{b}$ assumed to include ${ }^{137 \mathrm{~m}} \mathrm{Ba}$ in secular equilibrium

${ }^{\mathrm{c}}$ alpha + beta-gamma activity 
Table 8. Estimated Maximum Removable Contamination Levels - REC

\begin{tabular}{|c|c|c|c|c|c|}
\hline \multirow{2}{*}{ Radionuclide } & \multicolumn{5}{|c|}{ Removable Contamination $\left(\mu \mathrm{Ci} / 100 \mathrm{~cm}^{2}\right)$} \\
\hline & A-Cell & B-Cell & C-Cell & D-Cell & Airlock \\
\hline${ }^{54} \mathrm{Mn}$ & ND & ND & ND & ND & ND \\
\hline${ }^{60} \mathrm{Co}$ & 1.25E-04 & $6.50 E-02$ & 8.49E-04 & $1.31 E+00$ & $5.19 \mathrm{E}-03$ \\
\hline${ }^{79} \mathrm{Se}$ & $3.29 E-08$ & $1.53 E-04$ & 8.66E-07 & $1.79 E-04$ & 6.37E-06 \\
\hline${ }^{90} \mathrm{Sr}^{\mathrm{a}}$ & 1.47E-01 & $1.97 \mathrm{E}+02$ & $6.60 \mathrm{E}-01$ & $2.50 E+02$ & $1.55 \mathrm{E}+01$ \\
\hline${ }^{99} \mathrm{Tc}$ & 1.09E-06 & $5.08 \mathrm{E}-03$ & 2.87E-05 & $5.93 \mathrm{E}-03$ & $2.11 \mathrm{E}-04$ \\
\hline${ }^{108 \mathrm{~m}} \mathrm{Ag}$ & $\ldots$ & --- & --- & $1.59 \mathrm{E}-02$ & -- \\
\hline${ }^{125} \mathrm{Sb}$ & ND & ND & ND & ND & ND \\
\hline${ }^{134} \mathrm{Cs}$ & ND & $1.09 \mathrm{E}-01$ & $5.55 \mathrm{E}-03$ & $2.17 E-01$ & $1.46 \mathrm{E}-03$ \\
\hline${ }^{137} \mathrm{Cs}^{\mathrm{b}}$ & $3.46 \mathrm{E}-01$ & $3.29 \mathrm{E}+02$ & $1.10 \mathrm{E}+00$ & $1.25 E+03$ & $3.76 \mathrm{E}+01$ \\
\hline${ }^{154} \mathrm{Eu}$ & ND & 5.25E-01 & $6.98 \mathrm{E}-03$ & $6.99 \mathrm{E}+00$ & $1.72 \mathrm{E}-02$ \\
\hline${ }^{155} \mathrm{Eu}$ & ND & ND & 2.10E-03 & ND & ND \\
\hline${ }^{238} \mathrm{Pu}$ & $3.16 \mathrm{E}-05$ & $4.70 \mathrm{E}-02$ & $1.70 \mathrm{E}-03$ & $1.72 \mathrm{E}-01$ & $6.12 E-03$ \\
\hline${ }^{239} \mathrm{Pu}$ & ND & $9.04 \mathrm{E}-03$ & 3.61E-04 & 7.02E-02 & 2.19E-03 \\
\hline${ }^{240} \mathrm{Pu}$ & ND & 8.86E-03 & 3.53E-04 & $6.88 E-02$ & 2.15E-03 \\
\hline${ }^{241} \mathrm{Pu}$ & ND & 4.42E-01 & 1.76E-02 & $3.43 E+00$ & 1.07E-01 \\
\hline${ }^{242} \mathrm{Pu}$ & ND & $1.48 E-05$ & 5.91E-07 & 1.15E-04 & 3.59E-06 \\
\hline${ }^{241} \mathrm{Am}$ & 1.39E-04 & $6.49 \mathrm{E}-01$ & 3.67E-03 & 7.57E-01 & $2.70 \mathrm{E}-02$ \\
\hline${ }^{242} \mathrm{Cm}$ & ND & ND & ND & $5.75 \mathrm{E}-03$ & 9.01E-05 \\
\hline${ }^{243} \mathrm{Cm}$ & $1.25 \mathrm{E}-06$ & $1.18 \mathrm{E}-03$ & 4.15E-04 & 2.20E-02 & $1.40 E-04$ \\
\hline${ }^{244} \mathrm{Cm}$ & $8.60 \mathrm{E}-05$ & 8.11E-02 & 2.86E-02 & $1.52 E+00$ & 9.64E-03 \\
\hline Alpha & $1.48 E-03$ & $1.16 \mathrm{E}+00$ & 7.90E-02 & $2.28 E+00$ & 5.36E-02 \\
\hline Beta-Gamma & 4.32E-01 & $5.27 E+02$ & $1.77 \mathrm{E}+00$ & $1.51 E+03$ & $5.05 E+01$ \\
\hline Total Activity $^{c}$ & 4.32E-01 & $5.27 \mathrm{E}+02$ & $1.78 \mathrm{E}+00$ & $1.51 E+03$ & $5.06 \mathrm{E}+01$ \\
\hline
\end{tabular}

ND $=$ not detected

$\cdots=$ not reported

${ }^{a}$ assumed to include ${ }^{90} Y$ in secular equilibrium

b assumed to include ${ }^{137 \mathrm{~m}} \mathrm{Ba}$ in secular equilibrium

c alpha + beta-gamma activity 
Table 9. Estimated Maximum Total Contamination Levels - REC

\begin{tabular}{|c|c|c|c|c|c|}
\hline \multirow{2}{*}{ Radionuclide } & \multicolumn{5}{|c|}{ Total Contamination $\left(\mu \mathrm{Ci} / 100 \mathrm{~cm}^{2}\right)$} \\
\hline & A-Cell & B-Cell & C-Cell & D-Cell & Airlock \\
\hline${ }^{54} \mathrm{Mn}$ & ND & ND & ND & ND & ND \\
\hline${ }^{60} \mathrm{Co}$ & $3.75 E-03$ & $1.95 E+00$ & $2.55 \mathrm{E}-02$ & $3.93 E+01$ & $1.56 \mathrm{E}-01$ \\
\hline${ }^{79} \mathrm{Se}$ & $9.87 \mathrm{E}-07$ & $4.59 \mathrm{E}-03$ & $2.60 \mathrm{E}-05$ & $5.36 \mathrm{E}-03$ & $1.91 \mathrm{E}-04$ \\
\hline${ }^{90} \mathrm{Sr}^{\mathrm{a}}$ & $4.41 \mathrm{E}+00$ & $5.92 E+03$ & $1.98 \mathrm{E}+01$ & $7.50 E+03$ & $4.64 \mathrm{E}+02$ \\
\hline${ }^{99} \mathrm{Tc}$ & 3.27E-05 & 1.52E-01 & $8.62 E-04$ & 1.78E-01 & $6.34 \mathrm{E}-03$ \\
\hline${ }^{108 \mathrm{~m}} \mathrm{Ag}$ & --- & $\cdots$ & -- & 4.77E-01 & --- \\
\hline${ }^{125} \mathrm{Sb}$ & ND & ND & ND & ND & ND \\
\hline${ }^{134} \mathrm{Cs}$ & ND & $3.27 E+00$ & 1.67E-01 & $6.51 E+00$ & $4.38 \mathrm{E}-02$ \\
\hline${ }^{137} \mathrm{Cs}^{\mathrm{b}}$ & $1.04 \mathrm{E}+01$ & $9.87 E+03$ & $3.30 \mathrm{E}+01$ & $3.75 E+04$ & $1.13 \mathrm{E}+03$ \\
\hline${ }^{154} \mathrm{Eu}$ & ND & $1.58 \mathrm{E}+01$ & $2.09 \mathrm{E}-01$ & $2.10 \mathrm{E}+02$ & 5.16E-01 \\
\hline${ }^{155} \mathrm{Eu}$ & ND & ND & $6.30 \mathrm{E}-02$ & ND & ND \\
\hline${ }^{238} \mathrm{Pu}$ & $9.49 E-04$ & $1.41 E+00$ & $5.11 \mathrm{E}-02$ & $5.16 \mathrm{E}+00$ & $1.84 \mathrm{E}-01$ \\
\hline${ }^{239} \mathrm{Pu}$ & ND & $2.71 E-01$ & $1.08 \mathrm{E}-02$ & $2.11 E+00$ & $6.58 \mathrm{E}-02$ \\
\hline${ }^{240} \mathrm{Pu}$ & ND & $2.66 \mathrm{E}-01$ & $1.06 \mathrm{E}-02$ & $2.06 \mathrm{E}+00$ & $6.44 \mathrm{E}-02$ \\
\hline${ }^{241} \mathrm{Pu}$ & ND & $1.33 E+01$ & $5.29 \mathrm{E}-01$ & $1.03 E+02$ & $3.22 \mathrm{E}+00$ \\
\hline${ }^{242} \mathrm{Pu}$ & ND & $4.45 \mathrm{E}-04$ & 1.77E-05 & $3.45 E-03$ & $1.08 \mathrm{E}-04$ \\
\hline${ }^{241} \mathrm{Am}$ & 4.18E-03 & $1.95 E+01$ & 1.10E-01 & $2.27 E+01$ & 8.09E-01 \\
\hline${ }^{242} \mathrm{Cm}$ & ND & ND & ND & 1.73E-01 & 2.70E-03 \\
\hline${ }^{243} \mathrm{Cm}$ & 3.74E-05 & 3.53E-02 & 1.24E-02 & 6.61E-01 & $4.20 E-03$ \\
\hline${ }^{244} \mathrm{Cm}$ & 2.58E-03 & $2.43 E+00$ & 8.58E-01 & $4.55 E+01$ & 2.89E-01 \\
\hline Alpha & 4.44E-02 & $3.48 \mathrm{E}+01$ & $2.37 E+00$ & $6.84 E+01$ & $1.61 E+00$ \\
\hline Beta-Gamma & $1.29 \mathrm{E}+01$ & $1.58 \mathrm{E}+04$ & $5.30 E+01$ & $4.52 E+04$ & $1.52 E+03$ \\
\hline Total Activity ${ }^{c}$ & $1.30 E+01$ & $1.58 \mathrm{E}+04$ & $5.34 \mathrm{E}+01$ & $4.53 E+04$ & $1.52 E+03$ \\
\hline
\end{tabular}

ND $=$ not detected

-.- $=$ not reported

${ }^{a}$ assumed to include ${ }^{90} \mathrm{Y}$ in secular equilibrium

${ }^{b}$ assumed to include ${ }^{137 \mathrm{~m}} \mathrm{Ba}$ in secular equilibrium

${ }^{c}$ alpha + beta-gamma activity 


\section{REFERENCES}

Bechtel Hanford, (1998), "324/327 Buildings Stabilization/Deactivation Project Management Plan," HNF-IP-1289, Rev. 2, Bechtel Hanford, Richland, Washington.

Berk, R. W., Hill, R. L., and Landsman, S. D., (1998), "324 Building Physical and Radiological Characterization Study", Rev. A, IDMS Accession Number D199021302, pp. 182 to 319, B\&W Hanford Company, Richland, WA

Hill, R. L., (2001), "Radiological Characterization of the HLV Waste Treatment Skid in the 324 Facility," HNF-7699, Rev. 0, Fluor Hanford, Richland, Washington.

Hill, R. L., et. al., (2000), "Radiological Profile Methodology for Remote-Handled Transuranic Mixed Waste from 324 Facility B-Cell," HNF-6572, Rev. 1, Fluor Hanford, Richland, Washington.

Hill, R. L, and Hobart, R. L., (2000), "Data Analysis and Radionuclide Scaling Factor for the BCell Waste Stream," HNF-4904, Rev. 0-A, Fluor Hanford, Richland, Washington.

Mantooth, D. S., (2003), "Radiological Characterization of the Shielded Material Facility (SMF), CP-14484, Rev. 0, Fluor Hanford, Richland, Washington. 


\section{APPENDIX A}

\section{BASELINE RADIOLOGICAL CHARACTERIZATION DATA}

(TABULATED) 


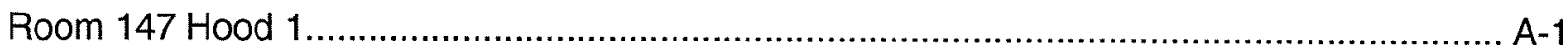

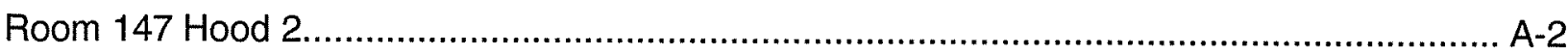

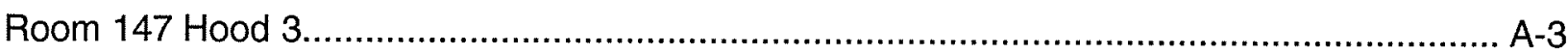

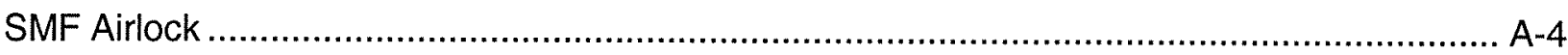

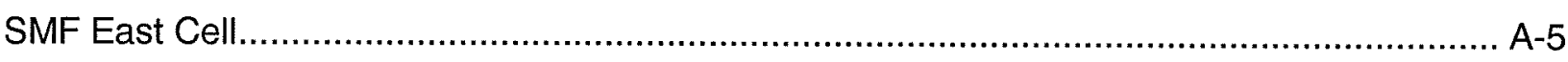

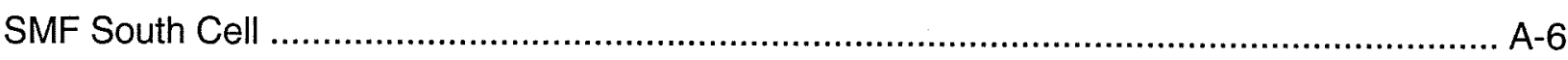

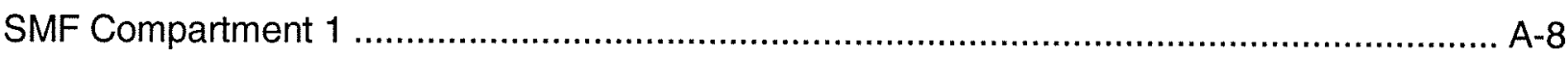

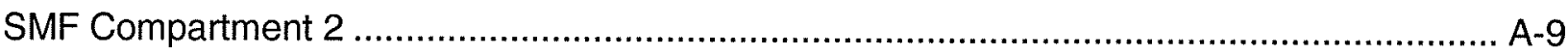

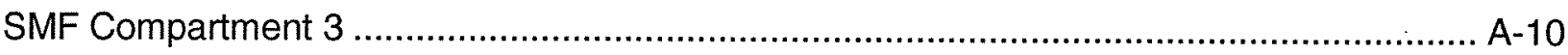

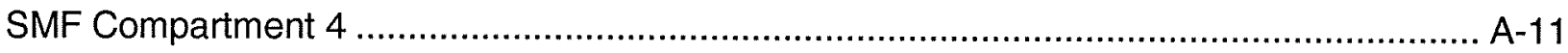

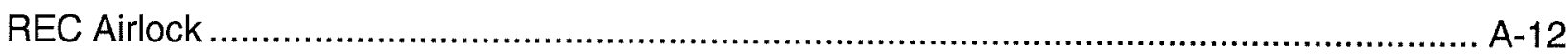

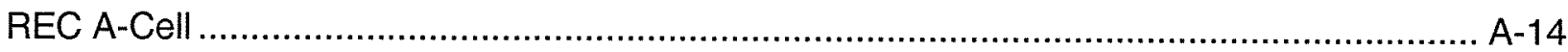

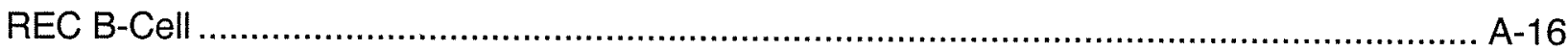

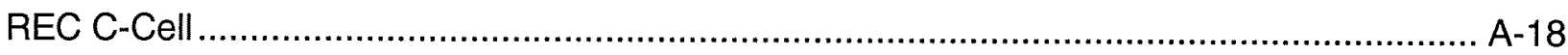

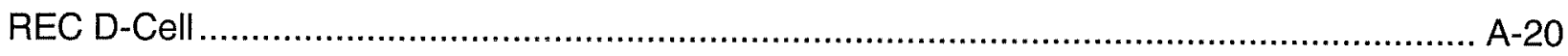




\section{Rm 147 Hood 1}

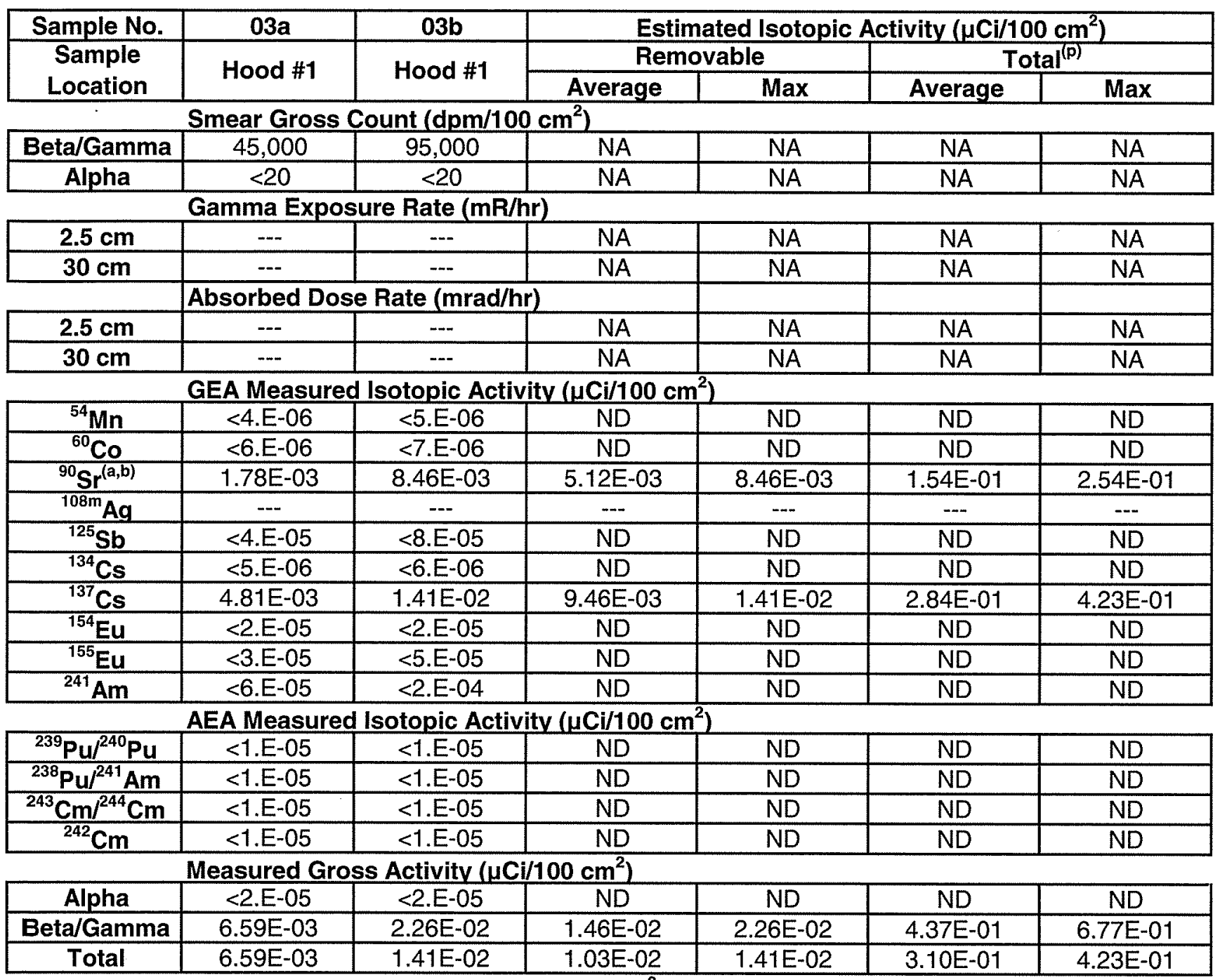

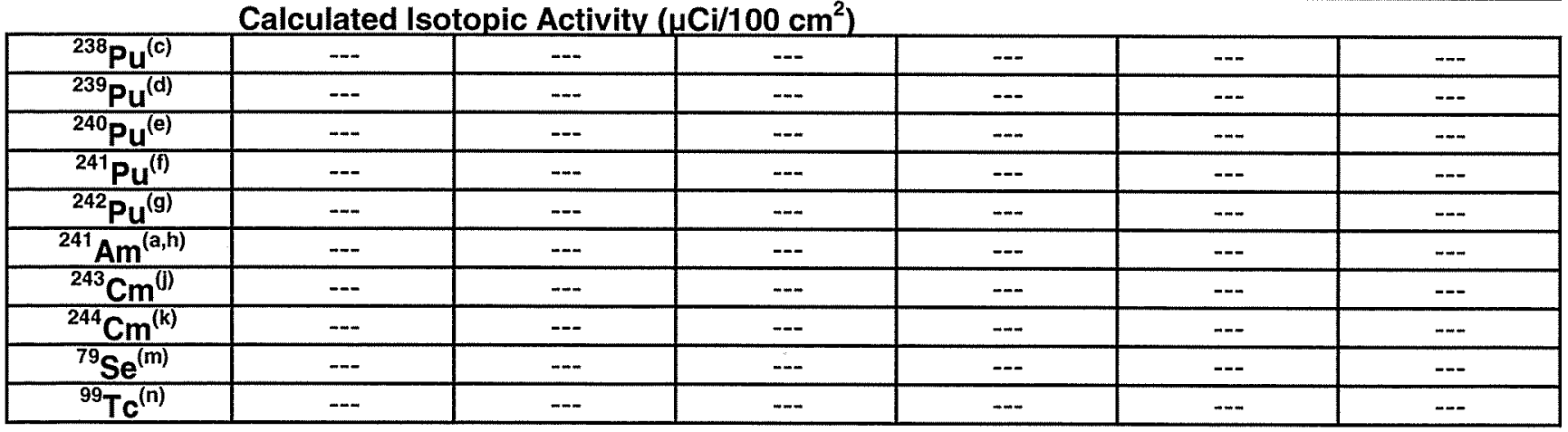

Assumptions:

(a) measured activity used, if available

(b) ${ }^{90} \mathrm{Sr}={ }^{137} \mathrm{Cs} \times 6.00 \mathrm{E}-01$

(c) ${ }^{238} \mathrm{Pu}={ }^{238} \mathrm{Pu} /{ }^{241} \mathrm{Am} \times 1.85 \mathrm{E}-01$
(d) ${ }^{239} \mathrm{Pu}={ }^{239 / 240} \mathrm{Pu} \times 5.05 \mathrm{E}-01$
(e) ${ }^{240} \mathrm{Pu}={ }^{239 / 240} \mathrm{Pu} \times 4.95 \mathrm{E}-01$
(f) ${ }^{241} \mathrm{Pu}={ }^{239 / 240} \mathrm{Pu} \times 2.47 \mathrm{E}+01$
(g) ${ }^{242} \mathrm{Pu}={ }^{239 / 240} \mathrm{Pu} \times 8.28 \mathrm{E}-04$

(h) ${ }^{241} \mathrm{Am}={ }^{238} \mathrm{Pu} /{ }^{241} \mathrm{Am} \times 8.15 \mathrm{E}-01$

(j) ${ }^{243} \mathrm{Cm}={ }^{243 / 244} \mathrm{Cm} \times 1.43 \mathrm{E}-02$

(k) ${ }^{244} \mathrm{Cm}={ }^{243 / 244} \mathrm{Cm} \times 9.86 \mathrm{E}-01$

(m) ${ }^{79} \mathrm{Se}={ }^{241} \mathrm{Am} \times 2.36 \mathrm{E}-04$

(n) ${ }^{99} \mathrm{Tc}={ }^{241} \mathrm{Am} \times 7.83 \mathrm{E}-03$

(p) total $=$ removable $\times 3.00 \mathrm{E}+01$ 


\section{Rm 147 Hood 2}

\begin{tabular}{|c|c|c|c|c|c|c|}
\hline \multirow{3}{*}{$\begin{array}{c}\text { Sample No. } \\
\text { Sample } \\
\text { Location }\end{array}$} & $05 \mathbf{a}$ & 05b & \multicolumn{4}{|c|}{ Estimated Isotopic Activity $\left(\mu \mathrm{Ci} / 100 \mathrm{~cm}^{2}\right)$} \\
\hline & \multirow{2}{*}{ Hood \#2 } & \multirow{2}{*}{ Hood \#2 } & \multicolumn{2}{|c|}{ Removable } & \multicolumn{2}{|c|}{ Total $^{(\mathrm{p})}$} \\
\hline & & & Average & Max & Average & $\operatorname{Max}$ \\
\hline \multicolumn{7}{|c|}{ Smear Gross Count (dpm/100 cm $\left.{ }^{2}\right)$} \\
\hline Beta/Gamma & 15,000 & 85,000 & NA & NA & NA & $\mathrm{NA}$ \\
\hline Alpha & 35 & $<20$ & $\mathrm{NA}$ & $\mathrm{NA}$ & NA & NA \\
\hline \multicolumn{7}{|c|}{ Gamma Exposure Rate (mR/hr) } \\
\hline $2.5 \mathrm{~cm}$ & -- & --- & $\mathrm{NA}$ & NA & $\mathrm{NA}$ & $\mathrm{NA}$ \\
\hline $30 \mathrm{~cm}$ & --- & $-\cdots$ & $\mathrm{NA}$ & $\mathrm{NA}$ & $\mathrm{NA}$ & $\mathrm{NA}$ \\
\hline \multicolumn{7}{|c|}{ Absorbed Dose Rate (mrad/hr) } \\
\hline $2.5 \mathrm{~cm}$ & --- & $-\cdots$ & NA & NA & NA & $\mathrm{NA}$ \\
\hline $30 \mathrm{~cm}$ & $\cdots$ & --- & $\mathrm{NA}$ & $\mathrm{NA}$ & NA & NA \\
\hline \multicolumn{7}{|c|}{ GEA Measured Isotopic Activity $\left(\mu \mathrm{Ci} / 100 \mathrm{~cm}^{2}\right)$} \\
\hline${ }^{54} \mathrm{Mn}$ & $<1 . \mathrm{E}-05$ & $<7 . \mathrm{E}-06$ & ND & ND & ND & ND \\
\hline${ }^{60} \mathrm{Co}$ & $9.11 \mathrm{E}-04$ & $1.61 \mathrm{E}-04$ & $5.36 \mathrm{E}-04$ & $9.11 E-04$ & $1.61 \mathrm{E}-02$ & $2.73 \mathrm{E}-02$ \\
\hline${ }^{90} \mathrm{Sr}^{(\mathrm{a}, \mathrm{b})}$ & $7.23 E-04$ & $1.16 \mathrm{E}-02$ & $6.15 \mathrm{E}-03$ & $1.16 \mathrm{E}-02$ & $1.85 \mathrm{E}-01$ & $3.47 \mathrm{E}-01$ \\
\hline${ }^{108 m} \mathrm{Ag}$ & --- & $-\cdots$ & $\cdots$ & -- & $-\cdots$ & --- \\
\hline${ }^{125} \mathrm{Sb}$ & $<4 . E-05$ & $<8 . E-05$ & ND & $\mathrm{ND}$ & ND & ND \\
\hline${ }^{134} \mathrm{Cs}$ & $<2 . E-05$ & $<8 . E-06$ & ND & ND & ND & ND \\
\hline${ }^{137} \mathrm{Cs}$ & $2.20 \mathrm{E}-03$ & $1.93 \mathrm{E}-02$ & $1.08 \mathrm{E}-02$ & $1.93 \mathrm{E}-02$ & $3.23 E-01$ & $5.79 E-01$ \\
\hline${ }^{154} \mathrm{Eu}$ & $<3 . E-05$ & $<3 . E-05$ & $\mathrm{ND}$ & ND & ND & ND \\
\hline${ }^{155} \mathrm{Eu}$ & $<2 . E-05$ & $<3 . E-05$ & $\mathrm{ND}$ & ND & ND & ND \\
\hline${ }^{241} \mathrm{Am}$ & $<2 . E-05$ & $<2 . E-05$ & ND & ND & ND & ND \\
\hline \multicolumn{7}{|c|}{ AEA Measured Isotopic Activity $\left(\mu \mathrm{Ci} / 100 \mathrm{~cm}^{2}\right)$} \\
\hline${ }^{239} \mathrm{Pu} /{ }^{240} \mathrm{Pu}$ & $<3 . \mathrm{E}-05$ & $<8 . \mathrm{E}-06$ & ND & ND & ND & ND \\
\hline${ }^{238} \mathrm{Pu} /{ }^{241} \mathrm{Am}$ & $<3 . \mathrm{E}-05$ & 9.31E-06 & $9.31 \mathrm{E}-06$ & 9.31E-06 & $2.79 \mathrm{E}-04$ & $2.79 \mathrm{E}-04$ \\
\hline${ }^{243} \mathrm{Cm} /{ }^{244} \mathrm{Cm}$ & $<3$. E-05 & $<4 . E-06$ & $\mathrm{ND}$ & $\mathrm{ND}$ & ND & ND \\
\hline${ }^{242} \mathrm{Cm}$ & $<3 . E-05$ & $<2 . E-06$ & ND & ND & ND & ND \\
\hline \multicolumn{7}{|c|}{ Measured Gross Activity $\left(\mu \mathrm{Ci} / 100 \mathrm{~cm}^{2}\right)$} \\
\hline Alpha & $<3 . \mathrm{E}-05$ & $1.49 \mathrm{E}-05$ & $1.49 E-05$ & $1.49 \mathrm{E}-05$ & 4.47E-04 & 4.47E-04 \\
\hline Beta/Gamma & 3.83E-03 & $3.10 \mathrm{E}-02$ & $1.74 \mathrm{E}-02$ & $3.10 \mathrm{E}-02$ & $5.23 \mathrm{E}-01$ & $9.31 \mathrm{E}-01$ \\
\hline Total & 3.83E-03 & $3.11 \mathrm{E}-02$ & $1.74 \mathrm{E}-02$ & $3.11 E-02$ & $5.23 E-01$ & $9.32 \mathrm{E}-01$ \\
\hline \multicolumn{7}{|c|}{ Calculated Isotopic Activity $\left(\mu \mathrm{Ci} / 100 \mathrm{~cm}^{2}\right)$} \\
\hline${ }^{238} \mathrm{Pu}^{(\mathrm{c})}$ & $-\cdots$ & $1.72 \mathrm{E}-06$ & $1.72 E-06$ & $1.72 \mathrm{E}-06$ & $5.17 \mathrm{E}-05$ & 5.17E-05 \\
\hline${ }^{239} \mathrm{Pu}^{(\mathrm{d})}$ & $\cdots$ & $\cdots$ & $\cdots$ & $\cdots$ & $\cdots$ & -- \\
\hline${ }^{240} \mathrm{Pu}^{(\mathrm{e})}$ & $\cdots$ & --- & -- & --- & $\cdots$ & $\cdots$ \\
\hline${ }^{241} \mathrm{Pu}^{(\mathrm{f})}$ & -- & $\cdots$ & $\cdots$ & $\cdots$ & -- & -- \\
\hline${ }^{242} \mathrm{Pu}^{(\mathrm{g})}$ & $\cdots$ & -- & -- & --- & $\cdots$ & $-\cdots$ \\
\hline${ }^{241} \mathrm{Am}^{(\mathrm{a}, \mathrm{h})}$ & $\cdots$ & $7.59 \mathrm{E}-06$ & $7.59 \mathrm{E}-06$ & 7.59E-06 & $2.28 \mathrm{E}-04$ & $2.28 \mathrm{E}-04$ \\
\hline${ }^{243} \mathrm{Cm}^{(0)}$ & -- & --- & $\cdots$ & --- & $--\cdot$ & --- \\
\hline${ }^{244} \mathrm{Cm}^{(\mathrm{k})}$ & $\cdots$ & $-\cdots$ & -- & $-\cdots$ & -- & $\cdots$ \\
\hline${ }^{79} \mathrm{Se}^{(\mathrm{m})}$ & -- & 1.79E-09 & 1.79E-09 & 1.79E-09 & 5.37E-08 & 5.37E-08 \\
\hline${ }^{99} \mathrm{Tc}^{(n)}$ & $\cdots$ & $5.94 E-08$ & $5.94 \mathrm{E}-08$ & $5.94 \mathrm{E}-08$ & $1.78 \mathrm{E}-06$ & $1.78 \mathrm{E}-06$ \\
\hline
\end{tabular}

Assumptions: (a) measured activity used, if available

(b) ${ }^{90} \mathrm{Sr}={ }^{137} \mathrm{Cs} \times 6.00 \mathrm{E}-01$

(c) ${ }^{238} \mathrm{Pu}={ }^{238} \mathrm{Pu} /{ }^{241} \mathrm{Am} \times 1.85 \mathrm{E}-01$

(d) ${ }^{239} \mathrm{Pu}={ }^{239 / 240} \mathrm{Pu} \times 5.05 \mathrm{E}-01$

(e) ${ }^{240} \mathrm{Pu}={ }^{239 / 240} \mathrm{Pu} \times 4.95 \mathrm{E}-01$

(f) ${ }^{241} \mathrm{Pu}={ }^{239 / 240} \mathrm{Pu} \times 2.47 \mathrm{E}+01$

(g) ${ }^{242} \mathrm{Pu}={ }^{239 / 240} \mathrm{Pu} \times 8.28 \mathrm{E}-04$ (h) ${ }^{241} \mathrm{Am}={ }^{238} \mathrm{Pu} /{ }^{241} \mathrm{Am} \times 8.15 \mathrm{E}-01$

(j) ${ }^{243} \mathrm{Cm}={ }^{243 / 244} \mathrm{Cm} \times 1.43 \mathrm{E}-02$

(k) ${ }^{244} \mathrm{Cm}={ }^{243 / 244} \mathrm{Cm} \times 9.86 \mathrm{E}-01$

(m) ${ }^{79} \mathrm{Se}={ }^{241} \mathrm{Am} \times 2.36 \mathrm{E}-04$

(n) ${ }^{99} \mathrm{Tc}={ }^{241} \mathrm{Am} \times 7.83 \mathrm{E}-03$

(p) total $=$ removable $\times 3.00 E+01$ 


\section{Rm 147 Hood 3}

\begin{tabular}{|c|c|c|c|c|c|}
\hline Sample No. & $04 a$ & $04 b$ & \multicolumn{3}{|c|}{ Estimated Isotopic Activity $\left(\mu \mathrm{Ci} / 100 \mathrm{~cm}^{2}\right)$} \\
\hline $\begin{array}{c}\text { Sample } \\
\text { Location }\end{array}$ & Hood \#3 & \multirow{2}{*}{ Hood \#3 } & \multicolumn{2}{|c|}{ Removable } & \multicolumn{2}{|c|}{ Total ${ }^{(p)}$} \\
\cline { 4 - 6 } & & Average & Max & Average & Max \\
\hline
\end{tabular}

\begin{tabular}{|c|c|c|c|c|c|c|}
\hline \multicolumn{7}{|c|}{ Smear Gross Count (dpm/100 $\left.\mathrm{cm}^{2}\right)$} \\
\hline Beta/Gamma & 15,000 & 12,500 & NA & NA & NA & NA \\
\hline Alpha & $<20$ & $<20$ & NA & NA & $\mathrm{NA}$ & NA \\
\hline \multicolumn{7}{|c|}{ Gamma Exposure Rate (mR/hr) } \\
\hline $2.5 \mathrm{~cm}$ & --- & $-\cdots$ & NA & NA & $\mathrm{NA}$ & $\overline{\mathrm{NA}}$ \\
\hline $30 \mathrm{~cm}$ & --- & $\cdots$ & NA & NA & NA & NA \\
\hline \multicolumn{7}{|c|}{ Absorbed Dose Rate (mrad/hr) } \\
\hline $2.5 \mathrm{~cm}$ & $-\cdots$ & $\cdots$ & NA & NA & NA & $\overline{\mathrm{NA}}$ \\
\hline $30 \mathrm{~cm}$ & $-\cdots$ & --- & $\overline{N A}$ & NA & NA & NA \\
\hline
\end{tabular}

\begin{tabular}{|c|c|c|c|c|c|c|}
\hline \multicolumn{7}{|c|}{ GEA Measured Isotopic Activity $\left(\mu \mathrm{Ci} / 100 \mathrm{~cm}^{2}\right)$} \\
\hline${ }^{54} \mathrm{Mn}$ & $<3 . E-06$ & $1.05 \mathrm{E}-05$ & $1.05 \mathrm{E}-05$ & $1.05 E-05$ & $3.15 \mathrm{E}-04$ & $3.15 E-04$ \\
\hline${ }^{60} \mathrm{Co}$ & $<4 . E-06$ & 5.97E-04 & 5.97E-04 & $5.97 E-04$ & $1.79 \mathrm{E}-02$ & $1.79 E-02$ \\
\hline${ }^{90} \mathrm{Sr}^{(\mathrm{a}, \mathrm{b})}$ & $2.58 \mathrm{E}-04$ & $1.44 \mathrm{E}-03$ & $8.49 E-04$ & 1.44E-03 & $2.55 \mathrm{E}-02$ & $4.32 E-02$ \\
\hline${ }^{108 m} \mathrm{Aq}$ & --- & --- & --- & $\cdots$ & -- & -- \\
\hline${ }^{125} \mathrm{Sb}$ & $<3 . E-05$ & $<3 . E-05$ & ND & ND & ND & ND \\
\hline${ }^{134} \mathrm{Cs}$ & $<4 . E-06$ & $<7 . \mathrm{E}-06$ & ND & ND & ND & ND \\
\hline${ }^{137} \mathrm{Cs}$ & $3.61 \mathrm{E}-03$ & $2.40 \mathrm{E}-03$ & $3.01 \mathrm{E}-03$ & $3.61 \mathrm{E}-03$ & $9.02 E-02$ & $1.08 \mathrm{E}-01$ \\
\hline${ }^{154} \mathrm{Eu}$ & $<9 . E-06$ & $<2 . E-05$ & ND & ND & ND & ND \\
\hline${ }^{155} \mathrm{Eu}$ & $<3 . E-05$ & $<3 . E-05$ & ND & ND & ND & ND \\
\hline${ }^{241} \mathrm{Am}$ & $<2 . E-04$ & $<2 . E-04$ & ND & ND & ND & ND \\
\hline
\end{tabular}

\begin{tabular}{|c|c|c|c|c|c|c|}
\hline${ }^{239} \mathrm{Pu} /{ }^{240} \mathrm{Pu}$ & $<3 . E-06$ & $<2 . E-05$ & $\mathrm{ND}$ & ND & ND & ND \\
\hline${ }^{238} \mathrm{Pu} /{ }^{241} \mathrm{Am}$ & $<3 . E-06$ & $<2 . E-05$ & ND & $\overline{N D}$ & ND & ND \\
\hline$\left.{ }^{243} \mathrm{Cm}\right|^{244} \mathrm{Cm}$ & $<3 . E-06$ & $<2 . E-05$ & ND & ND & ND & ND \\
\hline${ }^{242} \mathrm{Cm}$ & $<3 . E-06$ & $<2 . E-05$ & $\overline{N D}$ & $\mathrm{ND}$ & $\overline{N D}$ & $\mathrm{ND}$ \\
\hline
\end{tabular}

Measured Gross Activity $\left(\mu \mathrm{Ci} / 100 \mathrm{~cm}^{2}\right)$

\begin{tabular}{|c|c|c|c|c|c|c|}
\hline Alpha & $<2 . \mathrm{E}-05$ & $<2 . \mathrm{E}-05$ & $\mathrm{ND}$ & $\mathrm{ND}$ & $\mathrm{ND}$ & $\mathrm{ND}$ \\
\hline Beta/Gamma & $3.87 \mathrm{E}-03$ & $4.45 \mathrm{E}-03$ & $4.16 \mathrm{E}-03$ & $4.45 \mathrm{E}-03$ & $1.25 \mathrm{E}-01$ & $1.33 \mathrm{E}-01$ \\
\hline Total & $3.87 \mathrm{E}-03$ & $3.01 \mathrm{E}-03$ & $3.44 \mathrm{E}-03$ & $3.87 \mathrm{E}-03$ & $1.03 \mathrm{E}-01$ & $1.16 \mathrm{E}-01$ \\
\hline
\end{tabular}

\begin{tabular}{|c|c|c|c|c|c|c|}
\hline & \multicolumn{6}{|c|}{ 次 } \\
\hline${ }^{238} \mathrm{Pu}^{(\mathrm{c})}$ & $\cdots$ & $\cdots$ & $-\ldots$ & -- & $\cdots$ & $\cdots$ \\
\hline${ }^{239} \mathrm{Pu}^{\text {(d) }}$ & -- & $-\cdots$ & $\cdots$ & $\cdots$ & -- & $\ldots$ \\
\hline${ }^{240} \mathrm{Pu}^{(\mathrm{e})}$ & -- & --- & -- & $\ldots$ & -- & -- \\
\hline${ }^{241} \mathrm{Pu}^{(\mathrm{f})}$ & $\cdots$ & $\cdots$ & $\cdots$ & $\cdots$ & $\cdots$ & $\cdots$ \\
\hline${ }^{242} \mathrm{Pu}^{(\mathrm{g})}$ & -- & --- & --- & $\cdots$ & --- & $-\cdots$ \\
\hline $241 \mathrm{Am}^{(\mathrm{a}, \mathrm{h})}$ & $\cdots$ & $-\cdots$ & -- & -- & $\cdots$ & $\cdots$ \\
\hline${ }^{243} \mathrm{Cm}^{(\mathrm{i})}$ & - & $\cdots$ & $\cdots$ & $\cdots$ & $-\cdots$ & --- \\
\hline${ }^{244} \mathrm{Cm}^{(\mathrm{k})}$ & -- & --- & -- & --- & $\cdots$ & $\overline{---}$ \\
\hline${ }^{79} \mathrm{Se}^{(\mathrm{m})}$ & $\cdots$ & $\cdots$ & -- & -- & $\cdots$ & $\ldots$ \\
\hline${ }^{99} \mathrm{Tc}^{(n)}$ & -- & --- & --- & $\ldots$ & -- & -- \\
\hline
\end{tabular}

Assumptions:

(a) measured activity used, if available

(b) ${ }^{90} \mathrm{Sr}={ }^{137} \mathrm{Cs} \times 6.00 \mathrm{E}-01$

(c) ${ }^{238} \mathrm{Pu}={ }^{238} \mathrm{Pu} /{ }^{241} \mathrm{Am} \times 1.85 \mathrm{E}-01$
(d) ${ }^{239} \mathrm{Pu}={ }^{239 / 240} \mathrm{Pu} \times 5.05 \mathrm{E}-01$
(e) ${ }^{240} \mathrm{Pu}={ }^{239 / 240} \mathrm{Pu} \times 4.95 \mathrm{E}-01$
(f) ${ }^{241} \mathrm{Pu}={ }^{239 / 240} \mathrm{Pu} \times 2.47 \mathrm{E}+01$
(g) ${ }^{242} \mathrm{Pu}={ }^{239 / 240} \mathrm{Pu} \times 8.28 \mathrm{E}-04$

(h) ${ }^{241} \mathrm{Am}={ }^{238} \mathrm{Pu} /{ }^{241} \mathrm{Am} \times 8.15 \mathrm{E}-01$

(j) ${ }^{243} \mathrm{Cm}={ }^{243 / 244} \mathrm{Cm} \times 1.43 \mathrm{E}-02$

(k) ${ }^{244} \mathrm{Cm}={ }^{243 / 244} \mathrm{Cm} \times 9.86 \mathrm{E}-01$

(m) ${ }^{79} \mathrm{Se}={ }^{241} \mathrm{Am} \times 2.36 \mathrm{E}-04$

(n) ${ }^{99} \mathrm{Tc}={ }^{241} \mathrm{Am} \times 7.83 \mathrm{E}-03$

(p) total $=$ removable $\times 3.00 \mathrm{E}+01$ 


\section{SMF Airlock}

\begin{tabular}{|c|c|c|c|c|c|}
\hline \multirow{3}{*}{$\begin{array}{c}\text { Sample No. } \\
\text { Sample } \\
\text { Location }\end{array}$} & \multirow{3}{*}{$\begin{array}{c}38 b \\
\text { Ctr of Floor }\end{array}$} & \multicolumn{4}{|c|}{ Estimated Isotopic Activity $\left(\mu \mathrm{Ci} / 100 \mathrm{~cm}^{2}\right)^{(p)}$} \\
\hline & & \multicolumn{2}{|c|}{ Removable } & \multicolumn{2}{|c|}{ Total } \\
\hline & & Average & Max & Average & $\operatorname{Max}$ \\
\hline \multicolumn{6}{|c|}{ Smear Gross Count $\left(\mathrm{dpm} / 100 \mathrm{~cm}^{2}\right)$} \\
\hline Beta/Gamma & 2,000 & NA & $\mathrm{NA}$ & NA & $\mathrm{NA}$ \\
\hline Alpha & $<20$ & NA & NA & NA & NA \\
\hline \multicolumn{6}{|c|}{ Gamma Exposure Rate (mR/hr) } \\
\hline $2.5 \mathrm{~cm}$ & $<0.5$ & NA & NA & $\mathrm{NA}$ & $\mathrm{NA}$ \\
\hline $30 \mathrm{~cm}$ & $<0.5$ & NA & NA & $\mathrm{NA}$ & NA \\
\hline \multicolumn{6}{|c|}{ Absorbed Dose Rate (mrad/hr) } \\
\hline $2.5 \mathrm{~cm}$ & $<0.5$ & NA & NA & NA & $\overline{\mathrm{NA}}$ \\
\hline $30 \mathrm{~cm}$ & $<0.5$ & NA & $\mathrm{NA}$ & NA & NA \\
\hline \multicolumn{6}{|c|}{ GEA Measured Isotopic Activity $\left(\mu \mathrm{Ci} / 100 \mathrm{~cm}^{2}\right)$} \\
\hline${ }^{54} \mathrm{Mn}$ & $<3 . E-06$ & ND & ND & ND & ND \\
\hline${ }^{60} \mathrm{Co}$ & $<5 . E-06$ & ND & ND & ND & ND \\
\hline${ }^{90} \mathrm{Sr}^{(a)}$ & $-\cdots$ & -- & --- & $\cdots$ & $\cdots$ \\
\hline${ }^{108 m} \mathrm{Ag}$ & $-\cdots$ & --- & --- & --- & $-\cdots$ \\
\hline${ }^{125} \mathrm{Sb}$ & $<8 . E-06$ & ND & $\mathrm{ND}$ & ND & ND \\
\hline${ }^{134} \mathrm{Cs}$ & $<4 . \mathrm{E}-06$ & ND & ND & ND & ND \\
\hline${ }^{137} \mathrm{Cs}$ & $<4 . E-06$ & ND & ND & ND & ND \\
\hline${ }^{154} \mathrm{Eu}$ & $<9 . E-06$ & ND & ND & ND & ND \\
\hline${ }^{155} \mathrm{Eu}$ & $<2 . E-05$ & ND & ND & ND & ND \\
\hline${ }^{241} \mathrm{Am}$ & $<6 . E-05$ & ND & ND & ND & ND \\
\hline \multicolumn{6}{|c|}{ AEA Measured Isotopic Activity $\left(\mu \mathrm{Ci} / 100 \mathrm{~cm}^{2}\right)$} \\
\hline${ }^{239} \mathrm{Pu} /{ }^{240} \mathrm{Pu}$ & -- & -- & $-\cdots$ & $\cdots$ & $\overline{---}$ \\
\hline$\left.{ }^{238} \mathrm{Pu}\right|^{241} \mathrm{Am}$ & $\cdots$ & $-\cdots$ & $\cdots$ & $\cdots$ & $\overline{---}$ \\
\hline${ }^{243} \mathrm{Cm} /{ }^{244} \mathrm{Cm}$ & -- & $-\cdots$ & $\cdots$ & -- & $-\cdots$ \\
\hline${ }^{242} \mathrm{Cm}$ & --- & --- & $-\cdots$ & $-\cdots$ & $-\cdots$ \\
\hline \multicolumn{6}{|c|}{ Measured Gross Activity $\left(\mu \mathrm{Ci} / 100 \mathrm{~cm}^{2}\right)$} \\
\hline Alpha & $<2 . E-05$ & ND & ND & ND & ND \\
\hline Beta/Gamma & $\mathrm{ND}$ & ND & ND & ND & ND \\
\hline Total & ND & ND & ND & ND & ND \\
\hline \multicolumn{6}{|c|}{ Calculated Isotopic Activity $\left(\mu \mathrm{Ci} / 100 \mathrm{~cm}^{2}\right)$} \\
\hline${ }^{238} \mathrm{Pu}^{(\mathrm{c})}$ & $\ldots$ & -- & -- & $-\ldots$ & -- \\
\hline${ }^{239} \mathrm{Pu}^{\text {(d) }}$ & $\cdots$ & $-\cdots$ & $\cdots$ & --- & $\ldots$ \\
\hline${ }^{240} \mathrm{Pu}^{(\mathrm{e})}$ & $\cdots$ & $\cdots$ & $\cdots$ & $\cdots$ & $\cdots$ \\
\hline${ }^{241} \mathrm{Pu}^{(\mathrm{f})}$ & $\cdots$ & $-\cdots$ & $\overline{---}$ & $\cdots$ & --- \\
\hline${ }^{242} \mathrm{Pu}^{(\mathrm{g})}$ & -- & $-\cdots$ & $\ldots$ & $-\cdots$ & $\ldots$ \\
\hline${ }^{241} \mathrm{Am}^{(\mathrm{a}, \mathrm{h})}$ & $\overline{-\cdots}$ & $\overline{-\cdots}$ & $-\cdots$ & -- & $\overline{---}$ \\
\hline${ }^{243} \mathrm{Cm}^{(\mathrm{j})}$ & $-\cdots$ & $-\cdots$ & $\cdots$ & $\overline{---}$ & $-\cdots$ \\
\hline${ }^{244} \mathrm{Cm}^{(\mathrm{k})}$ & $\overline{-\cdots}$ & $\cdots$ & $\cdots$ & $-\cdots$ & $\cdots$ \\
\hline${ }^{79} \mathrm{Se}^{(\mathrm{m})}$ & $\cdots$ & $-\cdots$ & $\cdots$ & -- & $-\cdots$ \\
\hline${ }^{99} \mathrm{Tc}^{(\mathrm{n})}$ & --- & $\cdots$ & $\cdots$ & $\cdots$ & $\cdots$ \\
\hline
\end{tabular}

Assumptions: (a) measured activity used, if available

(c) ${ }^{238} \mathrm{Pu}={ }^{238} \mathrm{Pu} /{ }^{241} \mathrm{Am} \times 1.85 \mathrm{E}-01$
(d) ${ }^{239} \mathrm{Pu}={ }^{239 / 240} \mathrm{Pu} \times 5.05 \mathrm{E}-01$
(e) ${ }^{240} \mathrm{Pu}={ }^{239 / 240} \mathrm{Pu} \times 4.95 \mathrm{E}-01$
(f) ${ }^{241} \mathrm{Pu}={ }^{239 / 240} \mathrm{Pu} \times 2.47 \mathrm{E}+01$
(g) ${ }^{242} \mathrm{Pu}={ }^{239 / 240} \mathrm{Pu} \times 8.28 \mathrm{E}-04$

(h) ${ }^{241} \mathrm{Am}={ }^{238} \mathrm{Pu} /{ }^{241} \mathrm{Am} \times 8.15 \mathrm{E}-01$

(j) ${ }^{243} \mathrm{Cm}={ }^{243 / 244} \mathrm{Cm} \times 1.43 \mathrm{E}-02$

(k) ${ }^{244} \mathrm{Cm}={ }^{243 / 244} \mathrm{Cm} \times 9.86 \mathrm{E}-01$

(m) ${ }^{79} \mathrm{Se}={ }^{241} \mathrm{Am} \times 2.36 \mathrm{E}-04$

(n) ${ }^{99} \mathrm{Tc}={ }^{241} \mathrm{Am} \times 7.83 \mathrm{E}-03$

(p) total $=$ removable $\times 3.00 \mathrm{E}+01$ 


\section{SMF East Cell}

\begin{tabular}{|c|c|c|c|c|c|c|}
\hline \multirow{3}{*}{$\begin{array}{c}\text { Sample No. } \\
\text { Sample } \\
\text { Location }\end{array}$} & \multirow{3}{*}{\begin{tabular}{c|}
$32 \mathrm{a}$ \\
Ctr of Floor \\
\end{tabular}} & \multirow{3}{*}{$\begin{array}{c}32 b \\
\text { Ctr of Floor }\end{array}$} & \multicolumn{4}{|c|}{ Estimated Isotopic Activity $\left(\mu \mathrm{Ci} / 100 \mathrm{~cm}^{2}\right)$} \\
\hline & & & \multicolumn{2}{|c|}{ Removable } & \multicolumn{2}{|c|}{ Total $^{(p)}$} \\
\hline & & & Average & $\operatorname{Max}$ & Average & Max \\
\hline \multicolumn{7}{|c|}{ Smear Gross Count (dpm/100 $\left.\mathrm{cm}^{2}\right)$} \\
\hline Beta/Gamma & 5,500 & 3,500 & NA & NA & NA & NA \\
\hline Alpha & $<20$ & $<20$ & NA & NA & NA & NA \\
\hline \multicolumn{7}{|c|}{ Gamma Exposure Rate (mR/hr) } \\
\hline $2.5 \mathrm{~cm}$ & $<0.5$ & $<0.5$ & NA & NA & NA & NA \\
\hline $30 \mathrm{~cm}$ & $<0.5$ & $<0.5$ & NA & NA & NA & $\overline{N A}$ \\
\hline & \multicolumn{3}{|c|}{ Absorbed Dose Rate (mrad/hr) } & & & \\
\hline $2.5 \mathrm{~cm}$ & $<0.5$ & $<0.5$ & NA & NA & $\mathrm{NA}$ & NA \\
\hline $30 \mathrm{~cm}$ & $<0.5$ & $<0.5$ & NA & NA & NA & NA \\
\hline \multicolumn{7}{|c|}{ GEA Measured Isotopic Activity $\left(\mu \mathrm{Ci} / 100 \mathrm{~cm}^{2}\right)$} \\
\hline${ }^{54} \mathrm{Mn}$ & $<5 . \mathrm{E}-06$ & $<4 . \mathrm{E}-06$ & ND & ND & ND & ND \\
\hline${ }^{60} \mathrm{Co}$ & $1.81 E-05$ & $6.40 \mathrm{E}-06$ & 1.23E-05 & $1.81 E-05$ & $3.68 \mathrm{E}-04$ & $5.43 \mathrm{E}-04$ \\
\hline${ }^{90} \mathrm{Sr}^{(\mathrm{a})}$ & $-\cdots$ & $4.59 \mathrm{E}-02$ & $4.59 \mathrm{E}-02$ & $4.59 E-02$ & $1.38 \mathrm{E}+00$ & $1.38 E+00$ \\
\hline${ }^{108 m} \mathrm{Ag}$ & $\cdots$ & $-\cdots$ & - & -- & $-\cdots$ & -- \\
\hline${ }^{125} \mathrm{Sb}$ & $<3 . E-05$ & $<3 . E-05$ & $\mathrm{ND}$ & ND & ND & ND \\
\hline${ }^{134} \mathrm{Cs}$ & $<8 . E-06$ & $1.53 E-05$ & 1.53E-05 & 1.53E-05 & $4.59 \mathrm{E}-04$ & $4.59 E-04$ \\
\hline${ }^{137} \mathrm{Cs}$ & 1.53E-03 & $1.32 \mathrm{E}-03$ & 1.43E-03 & $1.53 \mathrm{E}-03$ & $4.28 \mathrm{E}-02$ & $4.59 \mathrm{E}-02$ \\
\hline${ }^{154} \mathrm{Eu}$ & $<2 . E-05$ & $<9 . \mathrm{E}-06$ & ND & ND & ND & ND \\
\hline${ }^{155} \mathrm{Eu}$ & $<2 . E-05$ & $<2 . E-05$ & ND & ND & ND & ND \\
\hline${ }^{241} \mathrm{Am}$ & $<9 . E-06$ & $<4 . E-05$ & ND & ND & ND & ND \\
\hline
\end{tabular}

\begin{tabular}{|c|c|c|c|c|c|c|}
\hline${ }^{239} \mathrm{Pu} /{ }^{240} \mathrm{Pu}$ & --- & --- & 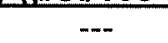 & -..- & --- & -.. \\
\hline${ }^{238} \mathrm{Pu} /{ }^{241} \mathrm{Am}$ & -.. & $\cdots$ & --- & --- & $\overline{---}$ & $\overline{---}$ \\
\hline${ }^{243} \mathrm{Cm} /{ }^{244} \mathrm{Cm}$ & -- & $\cdots$ & $\cdots$ & --- & -.. & $\ldots$ \\
\hline${ }^{242} \mathrm{Cm}$ & 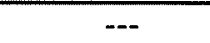 & --- & $\ldots$ & $\ldots$ & --- & --. \\
\hline \multicolumn{7}{|c|}{ Measured Gross Activity $\left(\mu \mathrm{Ci} / 100 \mathrm{~cm}^{2}\right)$} \\
\hline Alpha & $<2 . E-05$ & 1.03E-05 & $1.03 E-05$ & $1.03 \mathrm{E}-05$ & $3.09 \mathrm{E}-04$ & 3.09E-04 \\
\hline Beta/Gamma & $1.55 \mathrm{E}-03$ & $4.72 E-02$ & $2.44 \mathrm{E}-02$ & $4.72 \mathrm{E}-02$ & $7.32 \mathrm{E}-01$ & $1.42 E+00$ \\
\hline Total & $1.55 E-03$ & 4.73E-02 & $2.44 \mathrm{E}-02$ & $4.73 \mathrm{E}-02$ & $7.32 \mathrm{E}-01$ & $1.42 E+00$ \\
\hline
\end{tabular}

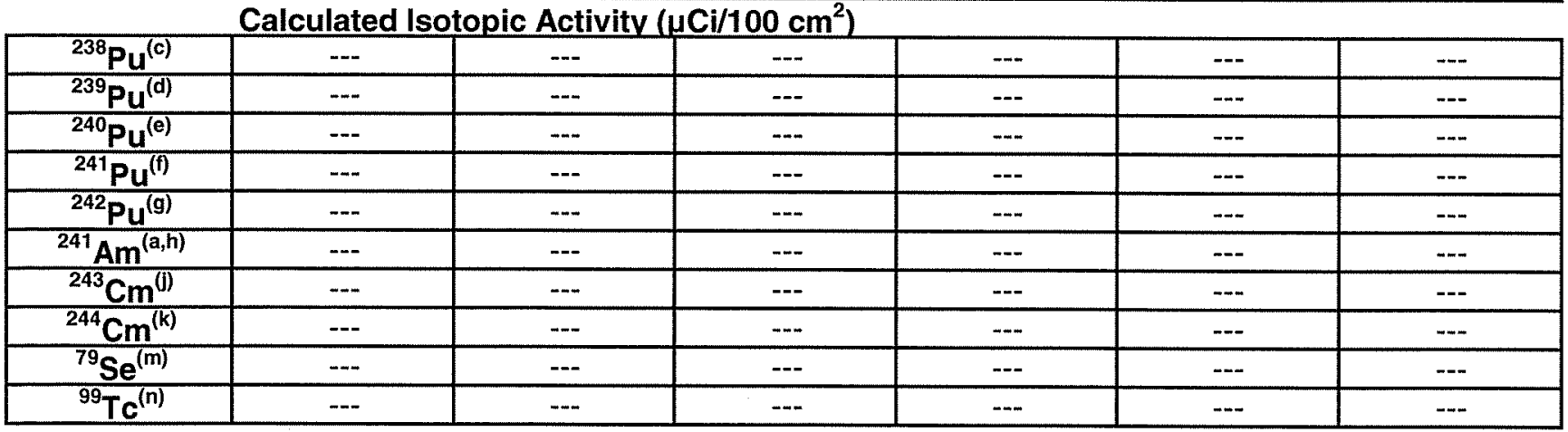

Assumptions:

(a) measured activity used, if available (c) ${ }^{238} \mathrm{Pu}={ }^{238} \mathrm{Pu} /{ }^{241} \mathrm{Am} \times 1.85 \mathrm{E}-01$
(d) ${ }^{239} \mathrm{Pu}={ }^{239 / 240} \mathrm{Pu} \times 5.05 \mathrm{E}-01$
(e) ${ }^{240} \mathrm{Pu}={ }^{239 / 240} \mathrm{Pu} \times 4.95 \mathrm{E}-01$
(f) ${ }^{241} \mathrm{Pu}={ }^{239 / 240} \mathrm{Pu} \times 2.47 \mathrm{E}+01$
(g) ${ }^{242} \mathrm{Pu}={ }^{239 / 240} \mathrm{Pu} \times 8.28 \mathrm{E}-04$

(h) ${ }^{241} \mathrm{Am}={ }^{238} \mathrm{Pu} /{ }^{241} \mathrm{Am} \times 8.15 \mathrm{E}-01$

(j) ${ }^{243} \mathrm{Cm}={ }^{243 / 244} \mathrm{Cm} \times 1.43 \mathrm{E}-02$

(k) ${ }^{244} \mathrm{Cm}={ }^{243 / 244} \mathrm{Cm} \times 9.86 \mathrm{E}-01$

(m) ${ }^{79} \mathrm{Se}={ }^{241} \mathrm{Am} \times 2.36 \mathrm{E}-04$

(n) ${ }^{99} \mathrm{Tc}={ }^{241} \mathrm{Am} \times 7.83 \mathrm{E}-03$

$(p)$ total $=$ removable $\times 3.00 E+01$ 


\section{SMF So Cell}

\begin{tabular}{|c|c|c|c|c|c|c|c|c|}
\hline Sample No. & $47 a$ & $47 b$ & $48 a$ & $48 b$ & $49 a$ & $49 b$ & $50 a$ & $50 \mathrm{~b}$ \\
\hline $\begin{array}{l}\text { Sample } \\
\text { Location }\end{array}$ & Station3 & Station 3 & Station 4 & Station 4 & Station 11 & Station 11 & Station 12 & Station 12 \\
\hline \multicolumn{9}{|c|}{ Smear Gross Count $\left(\mathrm{dpm} / 100 \mathrm{~cm}^{2}\right)$} \\
\hline Beta/Gamma & $-\cdots$ & $-\cdots$ & $\cdots$ & $\ldots$ & --- & $\overline{----}$ & --- & $\overline{---}$ \\
\hline Alpha & 700 & 700 & 350 & 350 & 350 & $<20$ & $<20$ & 350 \\
\hline \multicolumn{9}{|c|}{ Gamma Exposure Rate (mR/hr) } \\
\hline $2.5 \mathrm{~cm}$ & 40.5 & 42 & 6 & 3.5 & 3.8 & 1.5 & 5.3 & 9 \\
\hline $30 \mathrm{~cm}$ & 2 & 2.0 & 1.2 & 0.8 & 0.8 & 1 & 1 & 1.2 \\
\hline \multicolumn{9}{|c|}{ Absorbed Dose Rate (mrad/hr) } \\
\hline $2.5 \mathrm{~cm}$ & 6,860 & 8,658 & 444 & 237 & 416 & 50 & 235 & 471 \\
\hline $30 \mathrm{~cm}$ & 104 & 131 & 9.6 & 10.4 & 11.9 & 5.2 & 8.5 & 12.6 \\
\hline \multicolumn{9}{|c|}{ GEA Measured Isotopic Activity $\left(\mu \mathrm{Ci} / 100 \mathrm{~cm}^{2}\right)$} \\
\hline${ }^{54} \mathrm{Mn}$ & $1.34 \mathrm{E}-01$ & $1.25 E-01$ & $4.93 \mathrm{E}-02$ & $1.86 \mathrm{E}-02$ & $2.60 \mathrm{E}-02$ & $<4 . \mathrm{E}-03$ & $7.20 E-03$ & $3.70 \mathrm{E}-02$ \\
\hline${ }^{60} \mathrm{Co}$ & $1.34 E+01$ & $9.75 E+00$ & $2.77 E+00$ & $7.77 E-01$ & $2.70 \mathrm{E}-01$ & 1.67E-01 & $8.08 \mathrm{E}-01$ & $3.64 \mathrm{E}+00$ \\
\hline${ }^{90} \mathrm{Sr}^{(\mathrm{a})}$ & $8.34 \mathrm{E}+00$ & -- & $-\cdots$ & -- & --- & $-\cdots$ & -- & -- \\
\hline${ }^{108 \mathrm{~m}} \mathrm{Ag}$ & $-\cdots$ & -- & --- & $\cdots$ & --- & -- & --- & --- \\
\hline${ }^{125} \mathrm{Sb}$ & $<4 . E-01$ & $<5 . E-01$ & $<4 . \mathrm{E}-02$ & $<4 . E-02$ & $<3 . E-02$ & $<2 . E-02$ & $<3 . E-02$ & $<4 . E-02$ \\
\hline${ }^{134} \mathrm{Cs}$ & $2.21 \mathrm{E}+00$ & $2.14 \mathrm{E}+00$ & $2.76 \mathrm{E}-01$ & $4.24 \mathrm{E}-01$ & $1.62 \mathrm{E}-02$ & $1.07 \mathrm{E}-02$ & $6.78 \mathrm{E}-02$ & $2.44 \mathrm{E}-01$ \\
\hline${ }^{137} \mathrm{Cs}$ & $1.98 \mathrm{E}+02$ & $2.76 \mathrm{E}+02$ & $6.90 \mathrm{E}+00$ & $8.66 \mathrm{E}+00$ & $4.94 E+00$ & $1.21 \mathrm{E}+00$ & $3.38 \mathrm{E}+00$ & $4.98 \mathrm{E}+00$ \\
\hline${ }^{154} \mathrm{Eu}$ & $<7 . E-02$ & $<6 . \mathrm{E}-02$ & $<2 . E-02$ & $<8 . E-03$ & $<5 . E-03$ & $<5 . E-03$ & $<8 . E-03$ & $<2 . E-02$ \\
\hline${ }^{155} \mathrm{Eu}$ & $<3 . E-01$ & $<3 . E-01$ & $<2 . \mathrm{E}-02$ & $<2 . E-02$ & $<2$.E-02 & $<7$. E-03 & $<2 . E-02$ & $<2 . E-02$ \\
\hline${ }^{241} \mathrm{Am}$ & $<3 . \mathrm{E}-01$ & $<4 . E-01$ & $<3 . E-02$ & $<3 . E-02$ & $<2 . E-02$ & $<9 . E-03$ & $<2 . E-02$ & $<3 . E-02$ \\
\hline
\end{tabular}

\begin{tabular}{|c|c|c|c|c|c|c|c|c|}
\hline${ }^{239} \mathrm{Pu} /{ }^{240} \mathrm{Pu}$ & --- & $\cdots$ & $\ldots-$ & - & 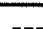 & 8 & $\pi$ & \\
\hline${ }^{238} \mathrm{Pu} /{ }^{241} \mathrm{Am}$ & $\cdots$ & --- & -- & -- &.-- & --- & --- & -- \\
\hline${ }^{243} \mathrm{Cm} /{ }^{244} \mathrm{Cm}$ & -- & $\cdots$ & --- & --- & $\cdots$ & -- & --- & $\cdots$ \\
\hline${ }^{242} \mathrm{Cm}$ & $\cdots$ & $\cdots$ & -- & -- & -- & $\cdots$ & $\cdots$ & $\cdots$ \\
\hline
\end{tabular}

Measured Gross Activity $\left(\mu \mathrm{Ci} / 100 \mathrm{~cm}^{2}\right)$

\begin{tabular}{|c|c|c|c|c|c|c|c|c|}
\hline Alpha & $2.14 \mathrm{E}-03$ & $-\cdots$ & $4.81 \mathrm{E}-03$ & -- & 1.25E-03 & $\cdots$ & 1.95E-03 & $--\cdot$ \\
\hline Beta/Gamma & $2.22 E+02$ & $2.88 \mathrm{E}+02$ & $1.00 \mathrm{E}+01$ & $9.88 E+00$ & $5.25 E+00$ & $1.39 E+00$ & $4.26 \mathrm{E}+00$ & $8.90 E+00$ \\
\hline Total & $2.22 E+02$ & $2.88 \mathrm{E}+02$ & $1.00 \mathrm{E}+01$ & $9.88 E+00$ & $5.25 \mathrm{E}+00$ & $1.39 E+00$ & $4.26 \mathrm{E}+00$ & $8.90 E+00$ \\
\hline \multicolumn{9}{|c|}{ Calculated Isotopic Activity $\left(\mu \mathrm{Ci} / 100 \mathrm{~cm}^{2}\right)$} \\
\hline${ }^{238} \mathrm{Pu}^{(c)}$ & -- & $\cdots$ & $-\cdots$ & -- & $\cdots$ & $\cdots$ & -- & --- \\
\hline${ }^{239} \mathrm{Pu}^{(\mathrm{d})}$ & $\cdots$ & -- & $\cdots$ & $-\cdots$ & -- & -- & --- & --- \\
\hline${ }^{240} \mathrm{Pu}^{(\mathrm{e})}$ & -- & $\cdots$ & -- & $-\cdots$ & $\cdots$ & $\cdots$ & $\cdots$ & $-\cdots$ \\
\hline${ }^{241} \mathrm{Pu}^{(t)}$ & $\cdots$ & -- & $\cdots$ & $\cdots$ & --- & $-\cdots$ & $-\cdots$ & --- \\
\hline${ }^{242} \mathrm{Pu}^{(\mathrm{g})}$ & -- & $\cdots$ & -- & $-\cdots$ & $\cdots$ & $\cdots$ & -- & $\cdots$ \\
\hline${ }^{241} \mathrm{Am}^{(\mathrm{a}, \mathrm{h})}$ & $\cdots$ & -- & $-\cdots$ & -- & -- & $-\cdots$ & $-\cdots$ & --- \\
\hline${ }^{243} \mathrm{Cm}^{(1)}$ & $-\cdots$ & -- & $\cdots$ & $\cdots$ & $\cdots$ & $-\cdots$ & -- & --- \\
\hline${ }^{244} \mathrm{Cm}^{(\mathrm{k})}$ & --- & -- & --- & --- & -- & --- & $-\cdots$ & -.- \\
\hline${ }^{79} \mathrm{Se}^{(\mathrm{m})}$ & $\cdots$ & --- & $\cdots$ & $\cdots$ & $\cdots$ & --- & $\cdots$ & --- \\
\hline${ }^{99} \mathrm{Tc}^{(\mathrm{n})}$ & -- & -- & --- & --- & $\cdots$ & $\cdots$ & $\cdots$ & $\cdots$ \\
\hline
\end{tabular}

Assumptions:

(a) measured activity used, if available

(c) ${ }^{238} \mathrm{Pu}={ }^{238} \mathrm{Pu} /{ }^{241} \mathrm{Am} \times 1.85 \mathrm{E}-01$
(d) ${ }^{239} \mathrm{Pu}={ }^{239 / 240} \mathrm{Pu} \times 5.05 \mathrm{E}-01$
(e) ${ }^{240} \mathrm{Pu}={ }^{239 / 240} \mathrm{Pu} \times 4.95 \mathrm{E}-01$
(f) ${ }^{241} \mathrm{Pu}={ }^{239 / 240} \mathrm{Pu} \times 2.47 \mathrm{E}+01$
(g) ${ }^{242} \mathrm{Pu}={ }^{239 / 240} \mathrm{Pu} \times 8.28 \mathrm{E}-04$

(h) ${ }^{241} \mathrm{Am}={ }^{238} \mathrm{Pu} /{ }^{241} \mathrm{Am} \times 8.15 \mathrm{E}-01$

(j) ${ }^{243} \mathrm{Cm}={ }^{243 / 244} \mathrm{Cm} \times 1.43 \mathrm{E}-02$

(k) ${ }^{244} \mathrm{Cm}={ }^{243 / 244} \mathrm{Cm} \times 9.86 \mathrm{E}-01$

(m) ${ }^{79} \mathrm{Se}={ }^{241} \mathrm{Am} \times 2.36 \mathrm{E}-04$

(n) ${ }^{99} \mathrm{Tc}={ }^{241} \mathrm{Am} \times 7.83 \mathrm{E}-03$

(p) total $=$ removable $\times 3.00 \mathrm{E}+01$ 


\section{SMF So Cell}

\begin{tabular}{|c|c|c|c|c|c|c|c|c|}
\hline \multirow{3}{*}{$\begin{array}{c}\text { Sample No. } \\
\text { Sample } \\
\text { Location }\end{array}$} & \multirow{3}{*}{ Station 10} & $51 b$ & $52 a$ & $52 b$ & \multicolumn{4}{|c|}{ Estimated Isotopic Activity $\left(\mu \mathrm{Ci} / 100 \mathrm{~cm}^{2}\right)^{(\mathrm{p})}$} \\
\hline & & \multirow{2}{*}{ Station 10} & \multirow{2}{*}{ Station 13} & \multirow{2}{*}{ Station 13} & \multicolumn{2}{|c|}{ Removable } & \multicolumn{2}{|c|}{ Total } \\
\hline & & & & & Average & Max & Average & Max \\
\hline \multicolumn{9}{|c|}{ Smear Gross Count $\left.(\mathrm{dpm} / 100 \mathrm{~cm})^{2}\right)$} \\
\hline Beta/Gamma & \begin{tabular}{|c|}
$-\cdot$ \\
\end{tabular} & \begin{tabular}{|l|}
--- \\
\end{tabular} & --- & --- & NA & NA & NA & NA \\
\hline Alpha & 350 & 350 & 350 & 1,400 & NA & NA & NA & NA \\
\hline \multicolumn{9}{|c|}{ Gamma Exposure Rate (mR/hr) } \\
\hline $2.5 \mathrm{~cm}$ & \begin{tabular}{|l|}
1.8 \\
\end{tabular} & 2.3 & 23 & 10.5 & NA & NA & NA & NA \\
\hline $30 \mathrm{~cm}$ & 1 & 0.7 & 1.5 & 1.2 & NA & $\mathrm{NA}$ & NA & $\mathrm{NA}$ \\
\hline \multicolumn{9}{|c|}{ Absorbed Dose Rate (mrad/hr) } \\
\hline $2.5 \mathrm{~cm}$ & 143 & 298 & 5,078 & 1,490 & NA & NA & NA & NA \\
\hline $30 \mathrm{~cm}$ & 8.5 & 10.6 & 87 & 27.6 & NA & $\mathrm{NA}$ & NA & NA \\
\hline \multicolumn{9}{|c|}{ GEA Measured Isotopic Activity $\left(\mu \mathrm{Ci} / 100 \mathrm{~cm}^{2}\right)$} \\
\hline${ }^{54} \mathrm{Mn}$ & $<2 . \mathrm{E}-03$ & $<2 . E-03$ & $1.20 \mathrm{E}-01$ & $1.93 \mathrm{E}-01$ & $7.89 E-02$ & $1.93 E-01$ & 2.37E+00 & $5.79 \mathrm{E}+00$ \\
\hline${ }^{60} \mathrm{Co}$ & $4.42 E-02$ & $3.14 \mathrm{E}-02$ & $1.89 \mathrm{E}+00$ & $2.53 E+00$ & $3.01 E+00$ & $1.34 E+01$ & $9.02 E+01$ & $4.02 E+02$ \\
\hline${ }^{90} \mathrm{Sr}^{(\mathrm{a})}$ & $-\cdots$ & $-\cdots$ & $2.32 E+00$ & --- & $5.33 E+00$ & $8.34 \mathrm{E}+00$ & $1.60 \mathrm{E}+02$ & $2.50 \mathrm{E}+02$ \\
\hline${ }^{108 \mathrm{~m}} \mathrm{Ag}$ & $\cdots$ & --- & $\cdots$ & --- & $-\cdots$ & $-\cdots$ & $-\cdots$ & --- \\
\hline${ }^{125} \mathrm{Sb}$ & $<2 . E-02$ & $<4 . E-02$ & $<4 . E-01$ & $<2 . E-01$ & ND & ND & ND & ND \\
\hline${ }^{134} \mathrm{Cs}$ & $5.33 E-03$ & 7.01E-03 & $1.52 \mathrm{E}-01$ & $1.15 \mathrm{E}-01$ & 4.72E-01 & $2.21 E+00$ & $1.42 E+01$ & $6.63 E+01$ \\
\hline${ }^{137} \mathrm{Cs}$ & $2.95 E+00$ & 9.77E+00 & $1.66 \mathrm{E}+02$ & $4.01 E+01$ & $6.02 E+01$ & $2.76 E+02$ & $1.81 E+03$ & $8.28 E+03$ \\
\hline${ }^{154} \mathrm{Eu}$ & $<4 . \mathrm{E}-03$ & $<4 . E-03$ & $<4 . E-02$ & $<3 . \mathrm{E}-02$ & ND & $\mathrm{ND}$ & $\mathrm{ND}$ & ND \\
\hline${ }^{155} \mathrm{Eu}$ & $<9 . E-03$ & $<2 . \mathrm{E}-02$ & $<3 . E-01$ & $<2 . E-01$ & ND & ND & ND & ND \\
\hline${ }^{241} \mathrm{Am}$ & $<2 . E-02$ & $<3 . E-02$ & $<4 . E-01$ & $<2 . E-01$ & $\mathrm{ND}$ & ND & ND & ND \\
\hline \multicolumn{9}{|c|}{ AEA Measured Isotopic Activity $\left(\mu \mathrm{Ci} / 100 \mathrm{~cm}^{2}\right)$} \\
\hline${ }^{239} \mathrm{Pu} /{ }^{240} \mathrm{Pu}$ & --- & $\cdots$ & $-\cdots$ & \begin{tabular}{|c|}
-- \\
\end{tabular} & $-\cdots$ & $-\cdots$ & $\cdots$ & $-\infty$ \\
\hline${ }^{238} \mathrm{Pu} /{ }^{241} \mathrm{Am}$ & $\cdots$ & -- & $-\cdots$ & --- & $-\cdots$ & $\cdots$ & $-\cdots$ & --- \\
\hline${ }^{243} \mathrm{Cm} /{ }^{244} \mathrm{Cm}$ & $-\cdots$ & $\cdots$ & --- & $\cdots$ & -- & $\overline{-\cdots}$ & $-\cdots$ & -- \\
\hline${ }^{242} \mathrm{Cm}$ & $-\cdots$ & $-\cdots$ & $-\cdots$ & $\cdots$ & $\cdots$ & $\cdots$ & $-\cdots$ & $\cdots$ \\
\hline \multicolumn{4}{|c|}{ Measured Gross Activity ( $\mu \mathrm{Ci} / 10 \mathrm{C}$} & $\cdots$ & $\cdots$ & $\cdots$ & --- & \\
\hline Alpha & $8.10 \mathrm{E}-04$ & --- & $3.03 E-03$ & $\cdots$ & 2.33E-03 & $4.81 E-03$ & 7.00E-02 & 1.44E-01 \\
\hline Beta/Gamma & $3.00 E+00$ & $9.81 \mathrm{E}+00$ & $1.70 \mathrm{E}+02$ & $4.29 E+01$ & $6.47 E+01$ & $2.88 E+02$ & 1.94E+03 & $8.64 E+03$ \\
\hline Total & $3.00 \mathrm{E}+00$ & $9.81 E+00$ & $1.70 \mathrm{E}+02$ & $4.29 E+01$ & $6.47 E+01$ & $2.88 E+02$ & $1.94 E+03$ & $8.64 \mathrm{E}+03$ \\
\hline \multicolumn{9}{|c|}{ Calculated Isotopic Activity $\left(\mu \mathrm{Ci} / 100 \mathrm{~cm}^{2}\right)$} \\
\hline${ }^{238} \mathrm{Pu}^{(\mathrm{c})}$ & \begin{tabular}{l|l}
-- \\
\end{tabular} & $-\cdots$ & $-\cdots$ & --- & $\cdots$ & -- & --- & $-\cdots$ \\
\hline${ }^{239} \mathrm{Pu}^{(\mathrm{d})}$ & $-\cdots$ & --- & $\cdots$ & $-\cdots$ & $\ldots$ & $\cdots$ & --- & $\overline{---}$ \\
\hline${ }^{240} \mathrm{Pu}^{(e)}$ & 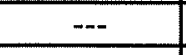 & $\cdots$ & $\cdots$ &.-- & $--\cdot$ & -- & $\cdots$ & $-\cdots$ \\
\hline${ }^{241} \mathrm{Pu}^{(\mathrm{f})}$ & $\cdots$ & --- & $\cdots$ & --- & $\cdots$ & $\cdots$ & $\cdots$ & -- \\
\hline${ }^{242} \mathrm{Pu}^{(\mathrm{g})}$ & $m$ & $\cdots$ & -- & $\cdots$ & $-\cdots$ & $\cdots$ & $-\cdots$ & $\cdots$ \\
\hline${ }^{241} \mathrm{Am}^{(\mathrm{a}, \mathrm{h})}$ & $-\cdots$ & $-\cdots$ & $\cdots$ & --- & $-\cdots$ & $\cdots$ & --- & $\cdots$ \\
\hline${ }^{243} \mathrm{Cm}^{(j)}$ & $\cdots$ & --- & -- & $\cdots$ & --- & $\cdots$ & -- & --- \\
\hline${ }^{244} \mathrm{Cm}^{(\mathrm{k})}$ & --- & -- & $\cdots$ & -- & $\cdots$ & -- & --- & $-\cdots$ \\
\hline${ }^{79} \mathrm{Se}^{(\mathrm{m})}$ & $\cdots$ & -- & $\cdots$ & --- & -- & $\cdots$ & -- & --- \\
\hline${ }^{99} \mathrm{Tc}^{(\mathrm{n})}$ & -- & $-\cdots$ & -- & $\cdots$ & $\cdots$ & --- & $\cdots$ & $\cdots$ \\
\hline
\end{tabular}

Assumptions:

(a) measured activity used, if available

(c) ${ }^{238} \mathrm{Pu}={ }^{238} \mathrm{Pu} /{ }^{241} \mathrm{Am} \times 1.85 \mathrm{E}-01$
(d) ${ }^{239} \mathrm{Pu}={ }^{239 / 240} \mathrm{Pu} \times 5.05 \mathrm{E}-01$
(e) ${ }^{240} \mathrm{Pu}={ }^{239 / 240} \mathrm{Pu} \times 4.95 \mathrm{E}-01$
(f) ${ }^{241} \mathrm{Pu}={ }^{239 / 240} \mathrm{Pu} \times 2.47 \mathrm{E}+01$
(g) ${ }^{242} \mathrm{Pu}={ }^{239 / 240} \mathrm{Pu} \times 8.28 \mathrm{E}-04$

(h) ${ }^{241} \mathrm{Am}={ }^{238} \mathrm{Pu} /{ }^{241} \mathrm{Am} \times 8.15 \mathrm{E}-01$

(j) ${ }^{243} \mathrm{Cm}={ }^{243 / 244} \mathrm{Cm} \times 1.43 \mathrm{E}-02$

(k) ${ }^{244} \mathrm{Cm}={ }^{243 / 244} \mathrm{Cm} \times 9.86 \mathrm{E}-01$

(m) ${ }^{79} \mathrm{Se}={ }^{241} \mathrm{Am} \times 2.36 \mathrm{E}-04$

(n) ${ }^{99} \mathrm{Tc}={ }^{241} \mathrm{Am} \times 7.83 \mathrm{E}-03$

(p) total $=$ removable $\times 3.00 E+01$ 


\section{SMF Comp 1}

\begin{tabular}{|c|c|c|c|c|c|c|c|}
\hline Sample No. & $60 \mathrm{a}$ & $60 \mathrm{~b}$ & $61 \mathrm{a}$ & $61 \mathrm{~b}$ & \multicolumn{3}{|c|}{ Estimated Isotopic Activity $\left(\mu \mathrm{Ci} / 100 \mathrm{~cm}^{2}\right)^{(\mathrm{p})}$} \\
\hline $\begin{array}{c}\text { Sample } \\
\text { Location }\end{array}$ & $\begin{array}{c}\text { Station 9 } \\
\text { Wall }\end{array}$ & $\begin{array}{c}\text { Station 9 } \\
\text { Wall }\end{array}$ & Station 8 & Station 8 & \multicolumn{2}{|c|}{ Removable } & \multicolumn{2}{|c|}{ Total } \\
\cline { 6 - 8 } & & & Average & Max & Average & Max \\
\hline
\end{tabular}

\begin{tabular}{|c|c|c|c|c|c|c|c|c|}
\hline \multicolumn{9}{|c|}{ Smear Gross Count (dpm/100 cm ${ }^{2}$ ) } \\
\hline Beta/Gamma & -- & $-\infty$ & -- & $\ldots$ & NA & NA & NA & NA \\
\hline Alpha & $<20$ & $<20$ & $<20$ & 350 & NA & $\mathrm{NA}$ & NA & $\overline{\mathrm{NA}}$ \\
\hline \multicolumn{9}{|c|}{ Gamma Exposure Rate (mR/hr) } \\
\hline $2.5 \mathrm{~cm}$ & 26 & 120 & 120 & 195 & NA & NA & NA & $\mathrm{NA}$ \\
\hline $30 \mathrm{~cm}$ & 2 & 4 & 5 & 7 & NA & NA & NA & NA \\
\hline \multicolumn{9}{|c|}{ Absorbed Dose Rate (mrad/hr) } \\
\hline $2.5 \mathrm{~cm}$ & 3,875 & 35,580 & 35,580 & 53,805 & NA & NA & NA & NA \\
\hline $30 \mathrm{~cm}$ & 56 & 352 & 471 & 646 & NA & NA & NA & NA \\
\hline \multicolumn{9}{|c|}{ GEA Measured Isotopic Activity $\left(\mathrm{uCi} / 100 \mathrm{~cm}^{2}\right)$} \\
\hline${ }^{54} \mathrm{Mn}$ & $<7 . \mathrm{E}-03$ & $<7 . E-02$ & $<6 . E-02$ & $<9 . E-02$ & ND & ND & ND & ND \\
\hline${ }^{60} \mathrm{Co}$ & $<4 . \mathrm{E}-03$ & $<2 . \mathrm{E}-02$ & $<1 . E-02$ & $<2 . E-02$ & ND & ND & ND & ND \\
\hline${ }^{90} \mathrm{Sr}^{(\mathrm{a})}$ & -- & $4.81 E+00$ & -- & $-\cdots$ & $4.81 E+00$ & $4.81 E+00$ & $1.44 \mathrm{E}+02$ & $1.44 E+02$ \\
\hline${ }^{108 m} \mathrm{Ag}$ & $-\cdots$ & $\cdots$ & $\cdots$ & -- & $\cdots$ & --- & -- & -- \\
\hline${ }^{125} \mathrm{Sb}$ & $<3 . E-01$ & $<7 . E-01$ & $<7 . E-01$ & $<9 . E-01$ & ND & ND & ND & ND \\
\hline${ }^{134} \mathrm{Cs}$ & $<1 . E-02$ & $<8 . E-02$ & $<7 . E-02$ & $<2 . E-01$ & ND & ND & ND & ND \\
\hline${ }^{137} \mathrm{Cs}$ & $7.66 \mathrm{E}+01$ & $7.73 E+02$ & $6.44 E+02$ & $1.04 \mathrm{E}+03$ & $6.33 \mathrm{E}+02$ & $1.04 E+03$ & $1.90 E+04$ & $3.12 E+04$ \\
\hline${ }^{154} \mathrm{Eu}$ & $<2 . E-02$ & $<1 . E-01$ & $<8 . E-02$ & $<2 . E-01$ & ND & ND & ND & ND \\
\hline${ }^{155} \mathrm{Eu}$ & $<2 . E-01$ & $<5 . E-01$ & $<5 . E-01$ & $<6$. E-01 & ND & ND & ND & ND \\
\hline${ }^{241} \mathrm{Am}$ & $<3 . E-01$ & $<8 . E-01$ & $<7 . \mathrm{E}-01$ & $<9 . \mathrm{E}-01$ & ND & ND & ND & ND \\
\hline
\end{tabular}

AEA Measured Isotopic Activity $\left(\mu \mathrm{Ci} / 100 \mathrm{~cm}^{2}\right)$

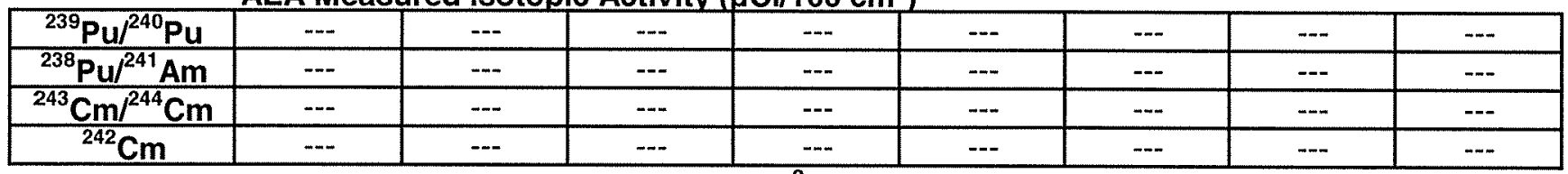

Measured Gross Activity $\left(\mu \mathrm{Ci} / 100 \mathrm{~cm}^{2}\right)$

\begin{tabular}{|c|c|c|c|c|c|c|c|c|}
\hline Alpha & --- & $1.25 \mathrm{E}-03$ & $4.06 \mathrm{E}-03$ & -- & $2.66 \mathrm{E}-03$ & $4.06 \mathrm{E}-03$ & $7.97 \mathrm{E}-02$ & $1.22 \mathrm{E}-01$ \\
\hline Beta/Gamma & $7.66 \mathrm{E}+01$ & $7.78 \mathrm{E}+02$ & $6.44 \mathrm{E}+02$ & $1.04 \mathrm{E}+03$ & $6.35 \mathrm{E}+02$ & $1.04 \mathrm{E}+03$ & $1.90 \mathrm{E}+04$ & $3.12 \mathrm{E}+04$ \\
\hline Total & $7.66 \mathrm{E}+01$ & $7.78 \mathrm{E}+02$ & $6.44 \mathrm{E}+02$ & $1.04 \mathrm{E}+03$ & $6.35 \mathrm{E}+02$ & $1.04 \mathrm{E}+03$ & $1.90 \mathrm{E}+04$ & $3.12 \mathrm{E}+04$ \\
\hline
\end{tabular}

\begin{tabular}{|c|c|c|c|c|c|c|c|c|}
\hline & & & & & & & & \\
\hline${ }^{238} \mathrm{Pu}^{(\mathrm{c})}$ & $\cdots$ & $\cdots$ & -- & $\cdots$ & -- & -- & $\cdots$ & --- \\
\hline${ }^{239} \mathrm{Pu}^{\text {(d) }}$ & -- & $\cdots$ & -- & $\cdots$ & -- & -- & --- & -- \\
\hline${ }^{240} \mathrm{Pu}^{(\mathrm{e})}$ & $\cdots$ & --- & -.- & --- & -- & $\cdots$ & $\cdots$ & $\cdots$ \\
\hline${ }^{241} \mathrm{Pu}^{(f)}$ & -- & $\cdots$ & $\cdots$ & $\cdots$ & $\cdots$ & $--\cdot$ & -- & -- \\
\hline${ }^{242} \mathrm{Pu}^{(\mathrm{g})}$ & $-\cdots$ & -- & $\cdots$ & --- & -- & $\cdots$ & $\cdots$ & $\cdots$ \\
\hline${ }^{241} \mathrm{Am}^{(\mathrm{a}, \mathrm{h})}$ & $\cdots$ & $\cdots$ & --- & -- & -- & --- & $\cdots$ & $\cdots$ \\
\hline${ }^{243} \mathrm{Cm}^{(0)}$ & --- & $\cdots$ & -- & -- & $\cdots$ & -- & $\cdots$ & -- \\
\hline${ }^{244} \mathrm{Cm}^{(\mathrm{k})}$ & $-\cdots$ & -- & $\ldots$ & -- & -- & -- & $\cdots$ & $\cdots$ \\
\hline${ }^{79} \mathrm{Se}^{(\mathrm{m})}$ & -- & $\cdots$ & -- & $\cdots$ & $\cdots$ & $\cdots$ & -- & $\cdots$ \\
\hline${ }^{99} \mathrm{Tc}^{(\mathrm{n})}$ & $-\cdots$ & - & $\ldots$ & -- & $\ldots$ & -- & $\ldots$ & $\ldots$ \\
\hline
\end{tabular}

Assumptions:

(a) measured activity used, if available

(c) ${ }^{238} \mathrm{Pu}={ }^{238} \mathrm{Pu} /{ }^{241} \mathrm{Am} \times 1.85 \mathrm{E}-01$
(d) ${ }^{239} \mathrm{Pu}={ }^{239 / 240} \mathrm{Pu} \times 5.05 \mathrm{E}-01$
(e) ${ }^{240} \mathrm{Pu}={ }^{239 / 240} \mathrm{Pu} \times 4.95 \mathrm{E}-01$
(f) ${ }^{241} \mathrm{Pu}={ }^{239 / 240} \mathrm{Pu} \times 2.47 \mathrm{E}+01$
(g) ${ }^{242} \mathrm{Pu}={ }^{239 / 240} \mathrm{Pu} \times 8.28 \mathrm{E}-04$

(h) ${ }^{241} \mathrm{Am}={ }^{238} \mathrm{Pu} /{ }^{241} \mathrm{Am} \times 8.15 \mathrm{E}-01$

(j) ${ }^{243} \mathrm{Cm}={ }^{243 / 244} \mathrm{Cm} \times 1.43 \mathrm{E}-02$

(k) ${ }^{244} \mathrm{Cm}={ }^{243 / 244} \mathrm{Cm} \times 9.86 \mathrm{E}-01$

(m) ${ }^{79} \mathrm{Se}={ }^{241} \mathrm{Am} \times 2.36 \mathrm{E}-04$

(n) ${ }^{99} \mathrm{Tc}={ }^{241} \mathrm{Am} \times 7.83 \mathrm{E}-03$

(p) total $=$ removable $\times 3.00 E+01$ 


\section{SMF Comp 2}

\begin{tabular}{|c|c|c|c|c|c|c|c|c|}
\hline \multirow{3}{*}{$\begin{array}{c}\text { Sample No. } \\
\text { Sample } \\
\text { Location }\end{array}$} & \multirow{3}{*}{$\frac{55 a}{\text { Floor }}$} & \multirow{3}{*}{$\begin{array}{c}55 b \\
\text { Floor }\end{array}$} & \multirow{3}{*}{$\frac{56 a}{E \text { Wall }}$} & \multirow{3}{*}{$\frac{56 b}{E \text { Wall }}$} & \multicolumn{4}{|c|}{ Estimated Isotopic Activity $\left(\mu \mathrm{Ci} / 100 \mathrm{~cm}^{2}\right)^{(p)}$} \\
\hline & & & & & \multicolumn{2}{|c|}{ Removable } & \multicolumn{2}{|c|}{ Total } \\
\hline & & & & & Average & Max & Average & Max \\
\hline \multicolumn{9}{|c|}{ Smear Gross Count (dpm/100 cm ${ }^{2}$ ) } \\
\hline Beta/Gamma & 200,000 & 225,000 & 50,000 & 60,000 & NA & NA & NA & NA \\
\hline Alpha & $<20$ & $<20$ & $<20$ & $<20$ & $\mathrm{NA}$ & NA & NA & $\mathrm{NA}$ \\
\hline \multicolumn{9}{|c|}{ Gamma Exposure Rate (mR/hr) } \\
\hline $2.5 \mathrm{~cm}$ & 1.2 & 1.2 & 1.5 & 1.0 & NA & $\mathrm{NA}$ & NA & NA \\
\hline $30 \mathrm{~cm}$ & 0.8 & 0.8 & 1.0 & 0.7 & $\mathrm{NA}$ & NA & NA & NA \\
\hline \multicolumn{9}{|c|}{ Absorbed Dose Rate (mrad/hr) } \\
\hline $2.5 \mathrm{~cm}$ & 4.8 & 5.1 & 3.0 & 3.4 & NA & NA & $\mathrm{NA}$ & NA \\
\hline $30 \mathrm{~cm}$ & 2.0 & 2.0 & 1.0 & 1.0 & NA & NA & $\overline{N A}$ & NA \\
\hline \multicolumn{9}{|c|}{ GEA Measured Isotopic Activity ( $\mu \mathrm{Ci} / 100 \mathrm{~cm}^{2}$ ) } \\
\hline${ }^{54} \mathrm{Mn}$ & $<8 . E-05$ & $<7 . E-05$ & $<7 . E-05$ & $<9 . E-05$ & ND & ND & ND & ND \\
\hline${ }^{60} \mathrm{Co}$ & $1.20 \mathrm{E}-03$ & 1.49E-03 & $1.23 \mathrm{E}-03$ & $1.36 \mathrm{E}-03$ & $1.32 \mathrm{E}-03$ & $1.49 \mathrm{E}-03$ & $3.96 \mathrm{E}-02$ & 4.47E-02 \\
\hline${ }^{90} \mathrm{Sr}^{(a, b)}$ & 1.30E-03 & --- & $\cdots$ & -- & $1.30 \mathrm{E}-03$ & $1.30 \mathrm{E}-03$ & $3.90 E-02$ & $3.90 E-02$ \\
\hline${ }^{108 m} \mathrm{Ag}$ & --- & $\cdots$ & --- & -- & $-\cdots$ & --- & -- & $-\cdots$ \\
\hline${ }^{125} \mathrm{Sb}$ & $<7 . E-04$ & $<7 . E-04$ & $<9 . E-04$ & $<8 . \mathrm{E}-04$ & ND & ND & ND & ND \\
\hline${ }^{134} \mathrm{Cs}$ & 1.36E-04 & $1.62 E-04$ & $1.83 E-04$ & $2.11 \mathrm{E}-04$ & 1.73E-04 & $2.11 E-04$ & $5.19 \mathrm{E}-03$ & $6.33 E-03$ \\
\hline${ }^{137} \mathrm{Cs}$ & $1.02 E-01$ & $9.35 \mathrm{E}-02$ & $1.49 E-01$ & $1.29 \mathrm{E}-01$ & $1.18 \mathrm{E}-01$ & $1.49 E-01$ & $3.55 E+00$ & $4.47 E+00$ \\
\hline${ }^{154} \mathrm{Eu}$ & $<2 . E-04$ & $<3 . E-04$ & $<2 . E-04$ & $<2 . E-04$ & ND & ND & ND & ND \\
\hline${ }^{155} \mathrm{Eu}$ & $<4 . E-04$ & $<4 . E-04$ & $<4 . E-04$ & $<4 . E-04$ & ND & ND & ND & ND \\
\hline${ }^{241} \mathrm{Am}$ & $<6 . \mathrm{E}-04$ & $<6 . \mathrm{E}-04$ & $<7 . E-04$ & $<6 . E-04$ & ND & ND & ND & ND \\
\hline \multicolumn{9}{|c|}{ AEA Measured Isotopic Activity $\left(\mu \mathrm{Ci} / 100 \mathrm{~cm}^{2}\right)$} \\
\hline${ }^{239} \mathrm{Pu} /{ }^{240} \mathrm{Pu}$ & $-\cdots$ & - & $\ldots$ & -- & --- & $\cdots$ & $-\cdots$ & $\cdots$ \\
\hline${ }^{238} \mathrm{Pu} /{ }^{241} \mathrm{Am}$ & $-\cdots$ & $-\cdots$ & $-\cdots$ & $-\cdots$ & $-\cdots$ & -- & 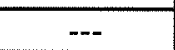 & $\cdots$ \\
\hline${ }^{243} \mathrm{Cm} /{ }^{244} \mathrm{Cm}$ & $-\cdots$ & $\cdots$ & $\cdots$ & $-\cdots$ & --- & --- & $\cdots$ & -- \\
\hline${ }^{242} \mathrm{Cm}$ & $\cdots$ & --- & -- & -- & -- & $-\cdots$ & $\cdots$ & $\cdots$ \\
\hline \multicolumn{9}{|c|}{ Measured Gross Activitv $\left(\mu \mathrm{Ci} / 100 \mathrm{~cm}^{2}\right)$} \\
\hline Alpha & $<5.00 \mathrm{E}-06$ & $\ldots$ & $<5.00 \mathrm{E}-06$ & -- & ND & ND & ND & ND \\
\hline Beta/Gamma & $1.05 \mathrm{E}-01$ & $9.52 E-02$ & $1.50 \mathrm{E}-01$ & $1.31 \mathrm{E}-01$ & $1.20 E-01$ & $1.50 E-01$ & $3.61 \mathrm{E}+00$ & $4.51 E+00$ \\
\hline Total & $1.05 \mathrm{E}-01$ & 9.52E-02 & $1.50 \mathrm{E}-01$ & $1.31 \mathrm{E}-01$ & $1.20 E-01$ & $1.50 \mathrm{E}-01$ & $3.61 \mathrm{E}+00$ & $4.51 E+00$ \\
\hline \multicolumn{9}{|c|}{ Calculated Isotopic Activity $\left(\mu \mathrm{Ci} / 100 \mathrm{~cm}^{2}\right)$} \\
\hline${ }^{238} \mathrm{Pu}^{(\mathrm{c})}$ & --- & --- & $\cdots$ & $\ldots$ & --- & $\cdots$ & $\cdots$ & $-\cdots$ \\
\hline${ }^{239} \mathrm{Pu}^{(\mathrm{d})}$ & $\cdots$ & $-\cdots$ & $\overline{-\cdots}$ & $\ldots$ & $-\cdots$ & --- & $\overline{---}$ & $\overline{---}$ \\
\hline${ }^{240} \mathrm{Pu}^{(\mathrm{e})}$ & $-\cdots$ & $-\cdots$ & $\overline{\cdots-}$ & $-\cdots$ & $\overline{-\cdots}$ & $\cdots$ & $\cdots$ & $-\cdots$ \\
\hline${ }^{241} \mathrm{Pu}^{(f)}$ & $\cdots$ & $-\cdots$ & $\cdots$ & $\cdots$ & $\cdots$ & -- & $\cdots$ & $\cdots$ \\
\hline${ }^{242} \mathrm{Pu}^{(\mathrm{g})}$ & $\cdots$ & $\cdots$ & $\cdots$ & $-\cdots$ & $\cdots$ & $\cdots$ & $-\cdots$ & $-\cdots$ \\
\hline${ }^{241} \mathrm{Am}^{(\mathrm{a}, \mathrm{h})}$ & $\cdots$ & $\cdots$ & $\cdots$ & -- & $-\cdots$ & $\cdots$ & $\cdots$ & $\cdots$ \\
\hline${ }^{243} \mathrm{Cm}^{(\mathrm{i})}$ & $\cdots$ & $\cdots$ & $-\cdots$ & $-\cdots$ & $\cdots$ & $\cdots$ & $-\cdots$ & -- \\
\hline${ }^{244} \mathrm{Cm}^{(\mathrm{k})}$ & $\cdots$ & $-\cdots$ & $\cdots$ & --- & $-\cdots$ & $\cdots$ & $\cdots$ & $\cdots$ \\
\hline${ }^{79} \mathrm{Se}^{(\mathrm{m})}$ & $-\cdots$ & $\cdots$ & $-\cdots$ & $\cdots$ & $\ldots$ & -- & -- & $\cdots$ \\
\hline${ }^{99} T c^{(n)}$ & $-\cdots$ & $-\cdots$ & $-\cdots$ & --- & $\cdots$ & $\cdots$ & $\cdots$ & --- \\
\hline
\end{tabular}

\section{Assumptions:}

(a) measured activity used, if available

(c) ${ }^{238} \mathrm{Pu}={ }^{238} \mathrm{Pu} /{ }^{241} \mathrm{Am} \times 1.85 \mathrm{E}-01$
(d) ${ }^{239} \mathrm{Pu}={ }^{239 / 240} \mathrm{Pu} \times 5.05 \mathrm{E}-01$
(e) ${ }^{240} \mathrm{Pu}={ }^{239 / 240} \mathrm{Pu} \times 4.95 \mathrm{E}-01$
(f) ${ }^{241} \mathrm{Pu}={ }^{239 / 240} \mathrm{Pu} \times 2.47 \mathrm{E}+01$
(g) ${ }^{242} \mathrm{Pu}={ }^{239 / 240} \mathrm{Pu} \times 8.28 \mathrm{E}-04$

(h) ${ }^{241} \mathrm{Am}={ }^{238} \mathrm{Pu} /{ }^{241} \mathrm{Am} \times 8.15 \mathrm{E}-01$

(j) ${ }^{243} \mathrm{Cm}={ }^{243 / 244} \mathrm{Cm} \times 1.43 \mathrm{E}-02$

(k) ${ }^{244} \mathrm{Cm}={ }^{243 / 244} \mathrm{Cm} \times 9.86 \mathrm{E}-01$

(m) ${ }^{79} \mathrm{Se}={ }^{241} \mathrm{Am} \times 2.36 \mathrm{E}-04$

(n) ${ }^{99} \mathrm{Tc}={ }^{241} \mathrm{Am} \times 7.83 \mathrm{E}-03$

(p) total $=$ removable $\times 3.00 \mathrm{E}+01$ 


\section{SMF Comp 3}

\begin{tabular}{|c|c|c|c|c|c|c|c|c|}
\hline \multirow{3}{*}{$\begin{array}{c}\text { Sample No. } \\
\text { Sample } \\
\text { Location }\end{array}$} & \multirow{3}{*}{$\begin{array}{c}57 a \\
\text { Table } \\
\end{array}$} & \multirow{3}{*}{$\frac{57 \mathrm{~b}}{\text { Table }}$} & \multirow{3}{*}{$\begin{array}{c}58 a \\
\text { Floor }\end{array}$} & \multirow{3}{*}{ Floor } & \multicolumn{4}{|c|}{ Estimated Isotopic Activity $\left(\mu \mathrm{Ci} / 100 \mathrm{~cm}^{2}\right)^{(\mathrm{p})}$} \\
\hline & & & & & \multicolumn{2}{|c|}{ Removable } & \multicolumn{2}{|c|}{ Total } \\
\hline & & & & & Average & Max & Average & $\operatorname{Max}$ \\
\hline \multicolumn{9}{|c|}{ Smear Gross Count $\left(\mathrm{dpm} / 100 \mathrm{~cm}^{2}\right)$} \\
\hline Beta/Gamma & 90,000 & 250,000 & 100,000 & 150,000 & NA & NA & NA & NA \\
\hline Alpha & $<20$ & $<20$ & $<20$ & $<20$ & NA & NA & NA & NA \\
\hline \multicolumn{9}{|c|}{ Gamma Exposure Rate (mR/hr) } \\
\hline $2.5 \mathrm{~cm}$ & 1.2 & 1.0 & 1.2 & 1.4 & NA & NA & NA & NA \\
\hline $30 \mathrm{~cm}$ & 0.7 & 0.7 & 0.8 & 0.9 & NA & NA & NA & NA \\
\hline \multicolumn{9}{|c|}{ Absorbed Dose Rate (mrad/hr) } \\
\hline $2.5 \mathrm{~cm}$ & 3.3 & 4.3 & 2.7 & 4.1 & NA & NA & NA & $\overline{N A}$ \\
\hline $30 \mathrm{~cm}$ & 1.9 & 2.2 & 1.4 & 3.6 & NA & NA & $\mathrm{NA}$ & NA \\
\hline \multicolumn{9}{|c|}{ GEA Measured Isotopic Activity $\left(\mu \mathrm{Ci} / 100 \mathrm{~cm}^{2}\right)$} \\
\hline${ }^{54} \mathrm{Mn}$ & $<1 . E-04$ & $3.59 \mathrm{E}-04$ & 1.77E-04 & $<2 . E-04$ & $2.68 \mathrm{E}-04$ & $3.59 E-04$ & $8.04 \mathrm{E}-03$ & $1.08 \mathrm{E}-02$ \\
\hline${ }^{60} \mathrm{Co}$ & $5.35 \mathrm{E}-03$ & $9.58 \mathrm{E}-03$ & $1.73 \mathrm{E}-03$ & $5.46 \mathrm{E}-03$ & $5.53 E-03$ & $9.58 \mathrm{E}-03$ & $1.66 \mathrm{E}-01$ & $2.87 E-01$ \\
\hline${ }^{90} \mathrm{Sr}^{(\mathrm{a})}$ & $1.09 E-03$ & --- & $-\cdots$ & $-\cdots$ & 1.09E-03 & 1.09E-03 & $3.27 E-02$ & $3.27 E-02$ \\
\hline${ }^{108 m} \mathrm{Ag}$ & $\cdots$ & $-\cdots$ & --- & --- & $-\cdots$ & $-\cdots$ & -- & $-\cdots$ \\
\hline${ }^{125} \mathrm{Sb}$ & $<5 . \mathrm{E}-04$ & $<1 . E-03$ & $<7$. E-04 & $<1 . \mathrm{E}-03$ & ND & ND & ND & ND \\
\hline${ }^{134} \mathrm{Cs}$ & $2.77 E-04$ & $5.63 \mathrm{E}-04$ & $1.50 \mathrm{E}-04$ & $3.64 \mathrm{E}-04$ & $3.39 E-04$ & $5.63 E-04$ & $1.02 E-02$ & $1.69 E-02$ \\
\hline${ }^{137} \mathrm{Cs}$ & $4.42 \mathrm{E}-02$ & $2.05 \mathrm{E}-01$ & $9.16 \mathrm{E}-02$ & $2.08 \mathrm{E}-01$ & $1.37 E-01$ & $2.08 \mathrm{E}-01$ & $4.12 E+00$ & $6.24 \mathrm{E}+00$ \\
\hline${ }^{154} \mathrm{Eu}$ & $<2 . E-04$ & $<2 . E-04$ & $<2 . E-04$ & $<3 . E-04$ & ND & ND & ND & ND \\
\hline${ }^{155} \mathrm{Eu}$ & $<3 . E-04$ & $<5 . E-04$ & $<4 . E-04$ & $<5 . E-04$ & ND & ND & $\mathrm{ND}$ & ND \\
\hline${ }^{241} \mathrm{Am}$ & $<4 . E-04$ & $<8 . E-04$ & $<6 . E-04$ & $<8 . E-04$ & ND & ND & ND & ND \\
\hline \multicolumn{9}{|c|}{ AEA Measured Isotopic Activity $\left(\mu \mathrm{Ci} / 100 \mathrm{~cm}^{2}\right)$} \\
\hline${ }^{239} \mathrm{Pu} /{ }^{240} \mathrm{Pu}$ & --- & -- & $\ldots$ & $-\cdots$ & $-\cdots$ & --- & $\cdots$ & $\cdots$ \\
\hline${ }^{238} \mathrm{Pu} /{ }^{241} \mathrm{Am}$ & $-\cdots$ & --- & $\cdots$ & $\cdots$ & $-\cdots$ & $-\cdots$ & $\cdots$ & $-\cdots$ \\
\hline${ }^{243} \mathrm{Cm} /{ }^{244} \mathrm{Cm}$ & $\cdots$ & $-\cdots$ & $-\cdots$ & -- & $-\cdots$ & $-\cdots$ & $-\cdots$ & $\cdots$ \\
\hline${ }^{242} \mathrm{Cm}$ & --- & $\cdots$ & $\cdots$ & $-\cdots$ & $-\cdots$ & -- & $-\cdots$ & --- \\
\hline \multicolumn{9}{|c|}{ Measured Gross Activity $\left(\mu \mathrm{Ci} / 100 \mathrm{~cm}^{2}\right)$} \\
\hline Alpha & $<5.00 e-06$ & $\cdots$ & $<5.00 \mathrm{E}-06$ & -- & ND & ND & ND & ND \\
\hline Beta/Gamma & $5.09 \mathrm{E}-02$ & $2.16 \mathrm{E}-01$ & 9.37E-02 & $2.14 \mathrm{E}-01$ & $1.43 \mathrm{E}-01$ & $2.16 \mathrm{E}-01$ & $4.30 \mathrm{E}+00$ & $6.47 \mathrm{E}+00$ \\
\hline Total & $5.09 \mathrm{E}-02$ & $2.16 E-01$ & 9.37E-02 & $2.14 \mathrm{E}-01$ & $1.43 E-01$ & $2.16 \mathrm{E}-01$ & $4.30 \mathrm{E}+00$ & $6.47 E+00$ \\
\hline \multicolumn{9}{|c|}{ Calculated Isotopic Activity $\left(\mu \mathrm{Ci} / 100 \mathrm{~cm}^{2}\right)$} \\
\hline${ }^{238} \mathrm{Pu}^{\text {(c) }}$ & -- & -- & -- & $\cdots$ & --- & --- & $-\cdots$ & $-\cdots$ \\
\hline${ }^{239} \mathrm{Pu}^{(\mathrm{d})}$ & $-\cdots$ & $-\cdots$ & $-\cdots$ & $-\cdots$ & $\overline{-\cdots}$ & $-\cdots$ & $\cdots$ & $\cdots$ \\
\hline${ }^{240} \mathrm{Pu}^{(\mathrm{e})}$ & -- & $\cdots$ & $-\cdots$ & -- & $-\cdots$ & $\cdots$ & $\cdots$ & -- \\
\hline${ }^{241} \mathrm{Pu}^{(\mathrm{f})}$ & $-\cdots$ & --- & $-\cdots$ & $\cdots$ & $\cdots$ & $-\cdots$ & $\cdots$ & $\cdots$ \\
\hline${ }^{242} \mathrm{Pu}^{(\mathrm{g})}$ & $\cdots$ & $\cdots$ & $-\cdots$ & --- & $\cdots$ & $\cdots$ & $\cdots$ & -- \\
\hline${ }^{241} \mathrm{Am}^{(\mathrm{a}, \mathrm{h})}$ & -- & $\cdots$ & $\cdots$ & $\cdots$ & $\cdots$ & -- & $-\cdots$ & $-\cdots$ \\
\hline${ }^{243} \mathrm{Cm}^{(0)}$ & $-\cdots$ & $-\cdots$ & -- & $\cdots$ & $\cdots$ & $-\cdots$ & $\cdots$ & $\cdots$ \\
\hline${ }^{244} \mathrm{Cm}^{(\mathrm{k})}$ & $\cdots$ & -- & $\cdots$ & $\cdots$ & --- & $\cdots$ & -- & $\cdots$ \\
\hline${ }^{79} \mathrm{Se}^{(\mathrm{m})}$ & $\cdots$ & $-\cdots$ & $\cdots$ & $\cdots$ & $\cdots$ & -- & $\cdots$ & $\cdots$ \\
\hline${ }^{99} T c^{(n)}$ & $\cdots$ & $\cdots$ & --- & -- & --- & $-\cdots$ & $-\cdots$ & $\cdots$ \\
\hline
\end{tabular}

Assumptions:

(a) measured activity used, if available

(c) ${ }^{238} \mathrm{Pu}={ }^{238} \mathrm{Pu} /{ }^{21} \mathrm{Am} \times 1.85 \mathrm{E}-01$
(d) ${ }^{239} \mathrm{Pu}={ }^{239 / 240} \mathrm{Pu} \times 5.05 \mathrm{E}-01$
(e) ${ }^{240} \mathrm{Pu}={ }^{239 / 240} \mathrm{Pu} \times 4.95 \mathrm{E}-01$
(f) ${ }^{241} \mathrm{Pu}={ }^{239 / 240} \mathrm{Pu} \times 2.47 \mathrm{E}+01$
(g) ${ }^{242} \mathrm{Pu}={ }^{239 / 240} \mathrm{Pu} \times$ 8.28E-04

(h) ${ }^{241} \mathrm{Am}={ }^{238} \mathrm{Pu} /{ }^{241} \mathrm{Am} \times 8.15 \mathrm{E}-01$

(j) ${ }^{243} \mathrm{Cm}={ }^{243 / 244} \mathrm{Cm} \times 1.43 \mathrm{E}-02$

(k) ${ }^{244} \mathrm{Cm}={ }^{243 / 244} \mathrm{Cm} \times 9.86 \mathrm{E}-01$

(m) ${ }^{79} \mathrm{Se}={ }^{241} \mathrm{Am} \times 2.36 \mathrm{E}-04$

(n) ${ }^{99} \mathrm{Tc}={ }^{241} \mathrm{Am} \times 7.83 \mathrm{E}-03$

$(p)$ total $=$ removable $\times 3.00 \mathrm{E}+01$ 


\section{SMF Comp 4}

\begin{tabular}{|c|c|c|c|c|c|c|c|c|}
\hline \multirow{3}{*}{$\begin{array}{c}\text { Sample No. } \\
\text { Sample } \\
\text { Location }\end{array}$} & \multirow{3}{*}{$\begin{array}{c}53 a \\
\text { E Wall }\end{array}$} & \multirow{3}{*}{$\begin{array}{c}53 b \\
\text { E Wall }\end{array}$} & \multirow{3}{*}{$\begin{array}{l}54 a \\
\text { Floor }\end{array}$} & \multirow{3}{*}{$\frac{54 b}{\text { Floor }}$} & \multicolumn{4}{|c|}{ Estimated Isotopic Activity $\left(\mu \mathrm{Ci} / 100 \mathrm{~cm}^{2}\right)^{(p)}$} \\
\hline & & & & & \multicolumn{2}{|c|}{ Removable } & \multicolumn{2}{|c|}{ Total } \\
\hline & & & & & Average & Max & Average & Max \\
\hline \multicolumn{9}{|c|}{ Smear Gross Count $\left(\mathrm{dpm} / 100 \mathrm{~cm}^{2}\right)$} \\
\hline Beta/Gamma & 200,000 & 125,000 & 450,000 & 350,000 & NA & NA & NA & $\overline{N A}$ \\
\hline Alpha & $<20$ & $<20$ & $<20$ & $<20$ & NA & NA & NA & NA \\
\hline \multicolumn{9}{|c|}{ Gamma Exposure Rate (mR/hr) } \\
\hline $2.5 \mathrm{~cm}$ & 1.2 & 1.0 & 1.4 & 1.2 & NA & $\mathrm{NA}$ & $\mathrm{NA}$ & NA \\
\hline $30 \mathrm{~cm}$ & 0.8 & 0.7 & 0.9 & 0.8 & NA & $\mathrm{NA}$ & NA & NA \\
\hline \multicolumn{9}{|c|}{ Absorbed Dose Rate (mrad/hr) } \\
\hline $2.5 \mathrm{~cm}$ & 3.6 & 6.4 & 6.5 & 5.4 & NA & NA & NA & $\mathrm{NA}$ \\
\hline $30 \mathrm{~cm}$ & 2.0 & 2.2 & 1.5 & 2.0 & NA & $\mathrm{NA}$ & NA & NA \\
\hline \multicolumn{9}{|c|}{ GEA Measured Isotopic Activity $\left(\mu \mathrm{Ci} / 100 \mathrm{~cm}^{2}\right)$} \\
\hline${ }^{54} \mathrm{Mn}$ & 1.84E-04 & $1.56 \mathrm{E}-04$ & $9.22 E-04$ & $4.08 \mathrm{E}-04$ & $4.18 \mathrm{E}-04$ & $9.22 \mathrm{E}-04$ & $1.25 \mathrm{E}-02$ & 2.77E-02 \\
\hline${ }^{60} \mathrm{Co}$ & 8.39E-03 & $5.60 \mathrm{E}-03$ & $3.21 E-02$ & $2.41 \mathrm{E}-02$ & $1.75 \mathrm{E}-02$ & $3.21 \mathrm{E}-02$ & $5.26 \mathrm{E}-01$ & $9.63 E-01$ \\
\hline${ }^{90} \mathrm{Sr}^{(\mathrm{a})}$ & $4.90 \mathrm{E}-03$ & $-\cdots$ & --- & -- & $4.90 E-03$ & $4.90 \mathrm{E}-03$ & $1.47 \mathrm{E}-01$ & $1.47 \mathrm{E}-01$ \\
\hline${ }^{108 \mathrm{~m}} \mathrm{Ag}$ & --- & $\cdots$ & --- & --- & --- & $\ldots$ & $\ldots$ & $\ldots$ \\
\hline${ }^{125} \mathrm{Sb}$ & $<6 . E-04$ & $<2 . E-03$ & 2.47E-03 & $<9 . \mathrm{E}-04$ & $2.47 E-03$ & $2.47 \mathrm{E}-03$ & $7.41 E-02$ & $7.41 E-02$ \\
\hline${ }^{134} \mathrm{Cs}$ & $4.51 E-04$ & $6.10 \mathrm{E}-04$ & $3.48 \mathrm{E}-03$ & $2.30 \mathrm{E}-03$ & $1.71 E-03$ & $3.48 E-03$ & $5.13 \mathrm{E}-02$ & $1.04 \mathrm{E}-01$ \\
\hline${ }^{137} \mathrm{Cs}$ & $5.85 \mathrm{E}-02$ & $2.19 \mathrm{E}-01$ & $1.35 \mathrm{E}-01$ & $1.29 \mathrm{E}-01$ & $1.35 \mathrm{E}-01$ & $2.19 \mathrm{E}-01$ & $4.06 \mathrm{E}+00$ & $6.57 \mathrm{E}+00$ \\
\hline${ }^{154} \mathrm{Eu}$ & $<2 . E-04$ & $<2 . E-04$ & $<3 . E-04$ & $<3 . E-04$ & ND & ND & ND & ND \\
\hline${ }^{155} \mathrm{Eu}$ & $<3$. E-04 & $<5 . E-04$ & $<5 . E-04$ & $<5$. E-04 & ND & ND & ND & ND \\
\hline${ }^{241} \mathrm{Am}$ & $<5 . \mathrm{E}-04$ & $<8 . E-04$ & $<7 . \mathrm{E}-04$ & $<7 . E-04$ & ND & ND & ND & ND \\
\hline \multicolumn{9}{|c|}{ AEA Measured Isotopic Activity $\left(\mu \mathrm{Ci} / 100 \mathrm{~cm}^{2}\right)$} \\
\hline${ }^{239} \mathrm{Pu} /{ }^{240} \mathrm{Pu}$ & $\ldots$ & -- & -- & $\ldots$ & $-\cdots$ & $\ldots$ & $\ldots$ & -- \\
\hline${ }^{238} \mathrm{Pu} /{ }^{241} \mathrm{Am}$ & $-\cdots$ & $\cdots$ & $-\cdots$ & $-\cdots$ & $\cdots$ & $\cdots$ & $\cdots$ & $\cdots$ \\
\hline${ }^{243} \mathrm{Cm} /{ }^{244} \mathrm{Cm}$ & $-\cdots$ & $\cdots$ & $\cdots$ & $-\cdots$ & -- & -- & -- & $-\cdots$ \\
\hline${ }^{242} \mathrm{Cm}$ & $\cdots$ & --- & --- & $\cdots$ & $-\cdots$ & $\cdots$ & $-\cdots$ & $-\cdots$ \\
\hline \multicolumn{9}{|c|}{ Measured Gross Activity $\left(\mu \mathrm{Ci} / 100 \mathrm{~cm}^{2}\right)$} \\
\hline Alpha & $<5.00 \mathrm{E}-06$ & $\cdots$ & $<5.00 \mathrm{E}-06$ & --- & ND & $\mathrm{ND}$ & ND & ND \\
\hline Beta/Gamma & $7.24 \mathrm{E}-02$ & $2.25 \mathrm{E}-01$ & $1.74 \mathrm{E}-01$ & $1.56 E-01$ & $1.57 E-01$ & $2.25 \mathrm{E}-01$ & $4.71 E+00$ & $6.76 E+00$ \\
\hline Total & $7.24 \mathrm{E}-02$ & $2.25 \mathrm{E}-01$ & $1.74 \mathrm{E}-01$ & $1.56 \mathrm{E}-01$ & 1.57E-01 & $2.25 E-01$ & $4.71 E+00$ & $6.76 E+00$ \\
\hline \multicolumn{9}{|c|}{ Calculated Isotopic Activitv $\left(\mu \mathrm{Ci} / 100 \mathrm{~cm}^{2}\right)$} \\
\hline${ }^{238} \mathrm{Pu}^{(\mathrm{c})}$ & $\cdots$ & -- & -- & $\ldots$ & $-\cdots$ & $-\ldots$ & $-\ldots$ & -- \\
\hline${ }^{239} \mathrm{Pu}^{(\mathrm{d})}$ & --- & $-\cdots$ & $-\cdots$ & $-\cdots$ & $\cdots$ & $\ldots$ & $\ldots$ & $-\ldots$ \\
\hline${ }^{240} \mathrm{Pu}^{(\mathrm{e})}$ & $-\cdots$ & $-\cdots$ & $\cdots$ & $-\cdots$ & $-\cdots$ & $\overline{---}$ & --- & -- \\
\hline${ }^{241} \mathrm{Pu}^{(f)}$ & $\cdots$ & $\cdots$ & -- & $-\cdots$ & $\cdots$ & $\cdots$ & $\cdots$ & $\ldots$ \\
\hline${ }^{242} \mathrm{Pu}^{(\mathrm{g})}$ & $-\cdots$ & -- & $\ldots$ & $-\cdots$ & $\cdots$ & -- & --- & -- \\
\hline${ }^{241} \mathrm{Am}^{(\mathrm{a}, \mathrm{h})}$ & $\cdots$ & --- & -- & $\cdots$ & $-\cdots$ & $-\cdots$ & $\cdots$ & $\cdots$ \\
\hline${ }^{243} \mathrm{Cm}^{(1)}$ & -- & $-\cdots$ & $\cdots$ & -- & $\cdots$ & $\cdots$ & -- & $\cdots$ \\
\hline${ }^{244} \mathrm{Cm}^{(\mathrm{k})}$ & -- & --- & $-\cdots$ & $-\cdots$ & $\cdots$ & $-\cdots$ & $-\cdots$ & -- \\
\hline${ }^{79} \mathrm{Se}^{(\mathrm{m})}$ & $\cdots$ & $\cdots$ & $\cdots$ & $\cdots$ & -- & $\cdots$ & $\cdots$ & $\ldots$ \\
\hline${ }^{99} \mathrm{Tc}^{(\mathrm{n})}$ & -- & $-\cdots$ & $\cdots$ & 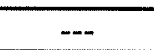 & $\cdots$ & -- & $-\ldots$ & -- \\
\hline
\end{tabular}

Assumptions: (a) measured activity used, if available

(c) ${ }^{238} \mathrm{Pu}={ }^{238} \mathrm{Pu} /{ }^{241} \mathrm{Am} \times 1.85 \mathrm{E}-01$
(d) ${ }^{239} \mathrm{Pu}={ }^{239 / 240} \mathrm{Pu} \times 5.05 \mathrm{E}-01$
(e) ${ }^{240} \mathrm{Pu}={ }^{239 / 240} \mathrm{Pu} \times 4.95 \mathrm{E}-01$
(f) ${ }^{241} \mathrm{Pu}={ }^{239 / 240} \mathrm{Pu} \times 2.47 \mathrm{E}+01$
(g) ${ }^{242} \mathrm{Pu}={ }^{239 / 240} \mathrm{Pu} \times 8.28 \mathrm{E}-04$

(h) ${ }^{241} \mathrm{Am}={ }^{238} \mathrm{Pu} /{ }^{241} \mathrm{Am} \times 8.15 \mathrm{E}-01$

(j) ${ }^{243} \mathrm{Cm}={ }^{243 / 244} \mathrm{Cm} \times 1.43 \mathrm{E}-02$

(k) ${ }^{244} \mathrm{Cm}={ }^{243 / 244} \mathrm{Cm} \times 9.86 \mathrm{E}-01$

(m) ${ }^{79} \mathrm{Se}={ }^{241} \mathrm{Am} \times 2.36 \mathrm{E}-04$

(n) ${ }^{99} \mathrm{Tc}={ }^{241} \mathrm{Am} \times 7.83 \mathrm{E}-03$

(p) total $=$ removable $\times 3.00 E+01$ 


\section{REC Airlock}

\begin{tabular}{|c|c|c|c|c|c|c|c|}
\hline Sample No. & $63 a$ & $63 b$ & $64 a$ & $64 b$ & $65 a$ & $65 b$ & $66 \mathbf{a}$ \\
\hline $\begin{array}{c}\text { Sample } \\
\text { Location }\end{array}$ & W Wall & W Wall & N wall door & $\mathrm{N}$ wall door & $\begin{array}{l}\text { Floor rt } \\
\text { side }\end{array}$ & $\begin{array}{l}\text { Floor rt } \\
\text { side }\end{array}$ & $\begin{array}{l}\text { Left side } \\
\text { door }\end{array}$ \\
\hline \multicolumn{8}{|c|}{ Smear Gross Count (dpm/100 cm $\left.{ }^{2}\right)$} \\
\hline Beta/Gamma & $-\cdots$ & --- & $-\cdots$ & $-\cdots$ & --- & $-\cdots$ & --- \\
\hline Alpha & 2,100 & 1,750 & 17,500 & 17,500 & 49,000 & 49,000 & 1,050 \\
\hline \multicolumn{8}{|c|}{ Gamma Exposure Rate (mR/hr) } \\
\hline $2.5 \mathrm{~cm}$ & 2.3 & 1.8 & 12.0 & 24.0 & 49.5 & 42.0 & 2.1 \\
\hline $30 \mathrm{~cm}$ & 0.7 & 0.7 & 1.0 & 1.1 & 1.5 & 1.5 & 0.8 \\
\hline \multicolumn{8}{|c|}{ Absorbed Dose Rate (mrad/hr) } \\
\hline $2.5 \mathrm{~cm}$ & 58 & 43 & 738 & 1,776 & 3,250 & 2,658 & 37 \\
\hline $30 \mathrm{~cm}$ & 3.1 & 3.1 & 16.0 & 21.8 & 48.0 & 42.0 & 2.6 \\
\hline \multicolumn{8}{|c|}{ GEA Measured Isotopic Activity $\left(\mu \mathrm{Ci} / 100 \mathrm{~cm}^{2}\right)$} \\
\hline${ }^{54} \mathrm{Mn}$ & $<6 . \mathrm{E}-4$ & $<6 . \mathrm{E}-04$ & $<9 . \mathrm{E}-04$ & $<9 . \mathrm{E}-04$ & $<2 . E-03$ & $<2 . \mathrm{E}-03$ & $<6 . E-04$ \\
\hline${ }^{60} \mathrm{Co}$ & $<8 . E-04$ & $<8 . \mathrm{E}-04$ & $<1 . E-03$ & $<1 . E-03$ & $2.25 \mathrm{E}-03$ & $<2 . \mathrm{E}-03$ & $<7 . E-04$ \\
\hline${ }^{90} \mathrm{Sr}^{(\mathrm{a}, \mathrm{b})}$ & $3.79 \mathrm{E}-01$ & $6.18 \mathrm{E}-01$ & $4.22 \mathrm{E}+00$ & $1.03 \mathrm{E}+01$ & $1.29 \mathrm{E}+01$ & $1.55 \mathrm{E}+01$ & $6.46 \mathrm{E}-01$ \\
\hline${ }^{108 \mathrm{~m}} \mathrm{Ag}$ & $\cdots$ & --- & $\cdots$ & $\cdots$ & --- & $\ldots$ & $\ldots$ \\
\hline${ }^{125} \mathrm{Sb}$ & $<7 . \mathrm{E}-03$ & $<8 . E-03$ & $<4 . E-02$ & $<5 . \mathrm{E}-02$ & $<7 . E-02$ & $<6 . \mathrm{E}-02$ & $<6 . \mathrm{E}-03$ \\
\hline${ }^{134} \mathrm{Cs}$ & $<2 . E-03$ & $<2 . \mathrm{E}-03$ & $<7 . E-03$ & $<9 . \mathrm{E}-03$ & $<2 . E-02$ & $<2 . E-02$ & $<2 . E-03$ \\
\hline${ }^{137} \mathrm{Cs}$ & $6.31 \mathrm{E}-01$ & $1.03 E+00$ & $9.34 E+00$ & $1.71 E+01$ & $3.76 \mathrm{E}+01$ & $2.58 \mathrm{E}+01$ & $4.42 E-01$ \\
\hline${ }^{154} \mathrm{Eu}$ & $<2 . E-03$ & $<2 . E-03$ & $5.54 \mathrm{E}-03$ & $<5 . E-03$ & $1.68 \mathrm{E}-02$ & $1.72 E-02$ & $<2 . E-03$ \\
\hline${ }^{155} \mathrm{Eu}$ & $<5$. E-03 & $<6 . E-03$ & $<3 . E-02$ & $<3 . E-02$ & $<5 . E-02$ & $<4 . E-02$ & $<5 . E-03$ \\
\hline${ }^{241} \mathrm{Am}$ & $<7 . \mathrm{E}-03$ & $<8 . E-03$ & $<3 . \mathrm{E}-02$ & $<3 . \mathrm{E}-02$ & $<5 . E-02$ & $<4 . E-02$ & $<6 . E-03$ \\
\hline \multicolumn{8}{|c|}{ AEA Measured Isotopic Activity $\left(\mu \mathrm{Ci} / 100 \mathrm{~cm}^{2}\right)$} \\
\hline${ }^{243} \mathrm{Am}$ & -- & $\cdots$ & $5.16 \mathrm{E}-05$ & -- & $5.83 \mathrm{E}-05$ & --- & $<2 . E-06$ \\
\hline${ }^{239} \mathrm{Pu} /{ }^{240} \mathrm{Pu}$ & $-\cdots$ & $-\cdots$ & $6.60 \mathrm{E}-04$ & $-\cdots$ & $4.34 \mathrm{E}-03$ & $\overline{---}$ & $4.91 E-04$ \\
\hline${ }^{238} \mathrm{Pu} /{ }^{241} \mathrm{Am}$ & $-\cdots$ & -- & $9.30 \mathrm{E}-03$ & $-\cdots$ & $3.31 E-02$ & $\cdots$ & $1.57 \mathrm{E}-03$ \\
\hline${ }^{243} \mathrm{Cm}^{244} \mathrm{Cm}$ & -- & $\cdots$ & $3.75 \mathrm{E}-03$ & $\cdots$ & $9.78 E-03$ & -- & $3.96 \mathrm{E}-04$ \\
\hline${ }^{242} \mathrm{Cm}$ & -- & $-\cdots$ & $4.09 \mathrm{E}-05$ & $-\cdots$ & $9.01 E-05$ & $-\cdots$ & $3.50 \mathrm{E}-06$ \\
\hline \multicolumn{8}{|c|}{ Measured Gross Activity $\left(\mu \mathrm{Ci} / 100 \mathrm{~cm}^{2}\right)$} \\
\hline Alpha & $4.54 \mathrm{E}-03$ & $\ldots$ & $1.38 \mathrm{E}-02$ & $1.35 \mathrm{E}-02$ & $4.74 \mathrm{E}-02$ & $5.36 \mathrm{E}-02$ & $2.46 \mathrm{E}-03$ \\
\hline Beta/Gamma & $1.01 E+00$ & $1.65 E+00$ & $1.36 \mathrm{E}+01$ & $2.74 E+01$ & $5.05 E+01$ & $4.13 E+01$ & $1.09 E+00$ \\
\hline Total & $1.01 E+00$ & $1.65 E+00$ & $1.36 \mathrm{E}+01$ & $2.74 \mathrm{E}+01$ & $5.06 E+01$ & $4.14 E+01$ & $1.09 E+00$ \\
\hline \multicolumn{8}{|c|}{ Calculated Isotopic Activity $\left(\mu \mathrm{Ci} / 100 \mathrm{~cm}^{2}\right)$} \\
\hline${ }^{238} \mathrm{Pu}^{(\mathrm{c})}$ & $\ldots$ & $-\cdots$ & $1.72 \mathrm{E}-03$ & $\overline{---}$ & $6.12 \mathrm{E}-03$ & --. & $2.90 \mathrm{E}-04$ \\
\hline${ }^{239} \mathrm{Pu}^{(\mathrm{d})}$ & $\cdots$ & $-\cdots$ & 3.33E-04 & $\cdots$ & $2.19 E-03$ & $\ldots$ & $2.48 \mathrm{E}-04$ \\
\hline${ }^{240} \mathrm{Pu}^{(\mathrm{e})}$ & -- & $-\cdots$ & $3.27 \mathrm{E}-04$ & $-\cdots$ & $2.15 \mathrm{E}-03$ & -- & $2.43 \mathrm{E}-04$ \\
\hline${ }^{241} \mathrm{Pu}^{(f)}$ & $\ldots$ & $\cdots$ & $1.63 \mathrm{E}-02$ & $\cdots$ & $1.07 \mathrm{E}-01$ & $\cdots$ & $1.21 \mathrm{E}-02$ \\
\hline${ }^{242} \mathrm{Pu}^{(\mathrm{g})}$ & --- & $\cdots$ & $5.46 \mathrm{E}-07$ & 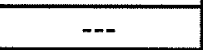 & $3.59 \mathrm{E}-06$ & $-\cdots$ & $4.07 \mathrm{E}-07$ \\
\hline${ }^{241} \mathrm{Am}^{(\mathrm{a}, \mathrm{h})}$ & $\cdots$ & --- & $7.58 \mathrm{E}-03$ & $-\cdots$ & $2.70 \mathrm{E}-02$ & $\cdots$ & $1.28 \mathrm{E}-03$ \\
\hline${ }^{243} \mathrm{Cm}^{(1)}$ & $-\cdots$ & $-\cdots$ & $5.36 \mathrm{E}-05$ & $\cdots$ & $1.40 \mathrm{E}-04$ & $\cdots$ & $5.66 \mathrm{E}-06$ \\
\hline${ }^{244} \mathrm{Cm}^{(\mathrm{k})}$ & --- & $\cdots$ & 3.70 E-03 & -- & $9.64 \mathrm{E}-03$ & $-\cdots$ & $3.90 \mathrm{E}-04$ \\
\hline${ }^{79} \mathrm{Se}^{(\mathrm{m})}$ & $\cdots$ & $\cdots$ & $1.79 \mathrm{E}-06$ & $-\cdots$ & $6.37 \mathrm{E}-06$ & $\ldots$ & $3.02 E-07$ \\
\hline${ }^{99} \mathrm{Tc}^{(\mathrm{n})}$ & --- & $\cdots$ & $5.93 \mathrm{E}-05$ & --- & $2.11 \mathrm{E}-04$ & --- & $1.00 \mathrm{E}-05$ \\
\hline
\end{tabular}

Assumptions: (a) measured activity used, if available

(b) ${ }^{90} \mathrm{Sr}={ }^{137} \mathrm{Cs} \times 6.00 \mathrm{E}-01$

(c) ${ }^{238} \mathrm{Pu}={ }^{238} \mathrm{Pu} /{ }^{241} \mathrm{Am} \times 1.85 \mathrm{E}-01$
(d) ${ }^{239} \mathrm{Pu}={ }^{239 / 240} \mathrm{Pu} \times 5.05 \mathrm{E}-01$
(e) ${ }^{240} \mathrm{Pu}={ }^{239 / 240} \mathrm{Pu} \times 4.95 \mathrm{E}-01$
(f) ${ }^{241} \mathrm{Pu}={ }^{239 / 240} \mathrm{Pu} \times 2.47 \mathrm{E}+01$
(g) ${ }^{242} \mathrm{Pu}={ }^{239 / 240} \mathrm{Pu} \times 8.28 \mathrm{E}-04$

(h) ${ }^{241} \mathrm{Am}={ }^{238} \mathrm{Pu} /{ }^{241} \mathrm{Am} \times 8.15 \mathrm{E}-01$

(j) ${ }^{243} \mathrm{Cm}={ }^{243 / 244} \mathrm{Cm} \times 1.43 \mathrm{E}-02$

(k) ${ }^{244} \mathrm{Cm}={ }^{243 / 244} \mathrm{Cm} \times 9.86 \mathrm{E}-01$

$(\mathrm{m}){ }^{79} \mathrm{Se}={ }^{241} \mathrm{Am} \times 2.36 \mathrm{E}-04$

(n) ${ }^{99} \mathrm{Tc}={ }^{241} \mathrm{Am} \times 7.83 \mathrm{E}-03$

(p) total $=$ removable $\times 3.00 \mathrm{E}+01$ 
REC Airlock

\begin{tabular}{|c|c|c|c|c|c|c|c|}
\hline Sample No. & $66 \mathrm{~b}$ & $67 \mathrm{a}$ & $67 \mathrm{~b}$ & \multicolumn{3}{|c|}{ Estimated Isotopic Activity $\left(\mu \mathrm{Ci} / 100 \mathrm{~cm}^{2}\right)^{(\mathrm{p})}$} \\
\hline $\begin{array}{c}\text { Sample } \\
\text { Location }\end{array}$ & $\begin{array}{c}\text { Left side } \\
\text { door }\end{array}$ & $\mathrm{s}$ Wall door & $\mathrm{s}$ Wall door & \multicolumn{2}{|c|}{ Removable } & \multicolumn{2}{|c|}{ Total } \\
\cline { 5 - 7 } & & Average & Max & Average & Max \\
\hline
\end{tabular}

\begin{tabular}{|c|c|c|c|c|c|c|c|}
\hline \multicolumn{8}{|c|}{ Smear Gross Count (dpm/100 $\left.\mathrm{cm}^{2}\right)$} \\
\hline Beta/Gamma & -- & -- & --- & $\mathrm{NA}$ & NA & NA & NA \\
\hline Alpha & 4,900 & 14,000 & 17,500 & NA & NA & NA & NA \\
\hline \multicolumn{8}{|c|}{ Gamma Exposure Rate (mR/hr) } \\
\hline $2.5 \mathrm{~cm}$ & 2.0 & 4.5 & 4.5 & NA & NA & NA & NA \\
\hline $30 \mathrm{~cm}$ & 0.7 & 0.9 & 0.9 & NA & NA & NA & NA \\
\hline \multicolumn{8}{|c|}{ Absorbed Dose Rate (mrad/hr) } \\
\hline $2.5 \mathrm{~cm}$ & 49 & 205 & 206 & NA & NA & $\overline{N A}$ & $\mathrm{NA}$ \\
\hline $30 \mathrm{~cm}$ & 3.1 & 6.3 & 5.1 & NA & NA & NA & NA \\
\hline \multicolumn{8}{|c|}{ GEA Measured Isotopic Activity $\left(\mu \mathrm{Ci} / 100 \mathrm{~cm}^{2}\right)$} \\
\hline${ }^{54} \mathrm{Mn}$ & $<6 . E-04$ & $<2 . E-03$ & $<1 . E-03$ & ND & ND & ND & ND \\
\hline${ }^{60} \mathrm{Co}$ & $<7 . E-04$ & $1.76 \mathrm{E}-03$ & $5.19 \mathrm{E}-03$ & $3.07 E-03$ & $5.19 E-03$ & $9.20 \mathrm{E}-02$ & $1.56 \mathrm{E}-01$ \\
\hline${ }^{90} \mathrm{Sr}^{(a, b)}$ & $4.09 E-01$ & $5.17 E+00$ & $2.02 E+00$ & $5.21 \mathrm{E}+00$ & $1.55 \mathrm{E}+01$ & $1.56 \mathrm{E}+02$ & $4.64 E+02$ \\
\hline${ }^{108 m} \mathrm{Ag}$ & $-\cdots$ & -- & $-\cdots$ & $-\cdots$ & -- & --- & $-\cdots$ \\
\hline${ }^{125} \mathrm{Sb}$ & $<7 . E-03$ & $<3 . E-02$ & $<3 . E-02$ & ND & ND & ND & ND \\
\hline${ }^{134} \mathrm{Cs}$ & $<2 . E-03$ & $1.46 \mathrm{E}-03$ & $<2 . E-03$ & $1.46 \mathrm{E}-03$ & $1.46 \mathrm{E}-03$ & $4.38 \mathrm{E}-02$ & $4.38 E-02$ \\
\hline${ }^{137} \mathrm{Cs}$ & $6.81 \mathrm{E}-01$ & $2.35 E+00$ & $3.36 \mathrm{E}+00$ & $9.83 E+00$ & $3.76 E+01$ & $2.95 E+02$ & $1.13 E+03$ \\
\hline${ }^{154} \mathrm{Eu}$ & $<2 . E-03$ & $<4 . E-03$ & $<5$. E-03 & $1.32 E-02$ & $1.72 E-02$ & $3.95 \mathrm{E}-01$ & $5.16 \mathrm{E}-01$ \\
\hline${ }^{155} \mathrm{Eu}$ & $<6 . \mathrm{E}-03$ & $<2 . \mathrm{E}-02$ & $<2 . E-02$ & ND & ND & ND & ND \\
\hline${ }^{241} \mathrm{Am}$ & $<7 . E-03$ & $<2 . E-02$ & $<2 . E-02$ & ND & ND & ND & ND \\
\hline
\end{tabular}

AEA Measured Isotopic Activity $\left(\mu \mathrm{Ci} / 100 \mathrm{~cm}^{2}\right)$

\begin{tabular}{|c|c|c|c|c|c|c|c|}
\hline${ }^{243} \mathrm{Am}$ & --- & $3.55 \mathrm{E}-05$ & -- & $4.85 \mathrm{E}-05$ & $5.83 \mathrm{E}-05$ & $1.45 \mathrm{E}-03$ & $1.75 \mathrm{E}-03$ \\
\hline${ }^{239} \mathrm{Pu} /{ }^{240} \mathrm{Pu}$ & --- & $8.11 \mathrm{E}-04$ & --- & $1.58 \mathrm{E}-03$ & $4.34 \mathrm{E}-03$ & $4.73 \mathrm{E}-02$ & $1.30 \mathrm{E}-01$ \\
\hline${ }^{238} \mathrm{Pu} /{ }^{241} \mathrm{Am}$ & --- & $4.64 \mathrm{E}-03$ & --- & $1.22 \mathrm{E}-02$ & $3.31 \mathrm{E}-02$ & $3.65 \mathrm{E}-01$ & $9.93 \mathrm{E}-01$ \\
\hline${ }^{243} \mathrm{Cm} /{ }^{244} \mathrm{Cm}$ & --- & $7.90 \mathrm{E}-03$ & --- & $5.46 \mathrm{E}-03$ & $9.78 \mathrm{E}-03$ & $1.64 \mathrm{E}-01$ & $2.93 \mathrm{E}-01$ \\
\hline${ }^{242} \mathrm{Cm}$ & $-\cdots$ & $1.60 \mathrm{E}-05$ & --- & $3.76 \mathrm{E}-05$ & $9.01 \mathrm{E}-05$ & $1.13 \mathrm{E}-03$ & $2.70 \mathrm{E}-03$ \\
\hline Alpha & $4.50 \mathrm{E}-03$ & $1.34 \mathrm{E}-02$ & $2.42 \mathrm{E}-02$ & $1.97 \mathrm{E}-02$ & $5.36 \mathrm{E}-02$ & $5.91 \mathrm{E}-01$ & $1.61 \mathrm{E}+00$ \\
\hline Beta/Gamma & $1.09 \mathrm{E}+00$ & $7.52 \mathrm{E}+00$ & $5.38 \mathrm{E}+00$ & $1.50 \mathrm{E}+01$ & $5.05 \mathrm{E}+01$ & $4.51 \mathrm{E}+02$ & $1.52 \mathrm{E}+03$ \\
\hline Total & $1.09 \mathrm{E}+00$ & $7.54 \mathrm{E}+00$ & $5.41 \mathrm{E}+00$ & $1.51 \mathrm{E}+01$ & $5.06 \mathrm{E}+01$ & $4.52 \mathrm{E}+02$ & $1.52 \mathrm{E}+03$ \\
\hline
\end{tabular}

\section{Calculated Isotopic Activity $\left(\mu \mathrm{Ci} / 100 \mathrm{~cm}^{2}\right)$}

\begin{tabular}{|c|c|c|c|c|c|c|c|}
\hline${ }^{238} \mathrm{Pu}^{(\mathrm{(c)}}$ & -- & $8.58 E-04$ & -- & $2.25 \mathrm{E}-03$ & $6.12 \mathrm{E}-03$ & $6.74 \mathrm{E}-02$ & $1.84 \mathrm{E}-01$ \\
\hline${ }^{239} \mathrm{Pu}^{\text {(d) }}$ & $\cdots$ & $4.10 E-04$ & $\cdots$ & $7.96 \mathrm{E}-04$ & $2.19 \mathrm{E}-03$ & $2.39 \mathrm{E}-02$ & $6.58 \mathrm{E}-02$ \\
\hline${ }^{240} \mathrm{Pu}^{(\mathrm{e})}$ & -- & $4.01 E-04$ & -- & $7.80 \mathrm{E}-04$ & $2.15 \mathrm{E}-03$ & $2.34 \mathrm{E}-02$ & $6.44 \mathrm{E}-02$ \\
\hline${ }^{241} \mathrm{Pu}^{(f)}$ & $\cdots$ & $2.00 E-02$ & $\cdots$ & $3.89 E-02$ & $1.07 \mathrm{E}-01$ & $1.17 E+00$ & $3.22 E+00$ \\
\hline${ }^{242} \mathrm{Pu}^{(\mathrm{g})}$ & -- & $6.72 \mathrm{E}-07$ & -- & $1.30 \mathrm{E}-06$ & $3.59 E-06$ & $3.91 E-05$ & $1.08 \mathrm{E}-04$ \\
\hline${ }^{241} \mathrm{Am}^{(\mathrm{a}, \mathrm{h})}$ & $\cdots$ & $3.78 \mathrm{E}-03$ & $-\cdots$ & $9.90 \mathrm{E}-03$ & $2.70 \mathrm{E}-02$ & 2.97E-01 & 8.09E-01 \\
\hline${ }^{243} \mathrm{Cm}^{(0)}$ & $\cdots$ & $1.13 \mathrm{E}-04$ & $\cdots$ & 7.80 E-05 & $1.40 \mathrm{E}-04$ & $2.34 \mathrm{E}-03$ & $4.20 E-03$ \\
\hline${ }^{244} \mathrm{Cm}^{(\mathrm{k})}$ & --- & $7.79 \mathrm{E}-03$ & - & $5.38 E-03$ & $9.64 \mathrm{E}-03$ & 1.61E-01 & $2.89 E-01$ \\
\hline${ }^{79} \mathrm{Se}^{(\mathrm{m})}$ & $\cdots$ & $8.92 E-07$ & $\cdots$ & $2.34 \mathrm{E}-06$ & $6.37 E-06$ & $7.01 \mathrm{E}-05$ & $1.91 \mathrm{E}-04$ \\
\hline${ }^{99} \mathrm{Tc}^{(\mathrm{n})}$ & --- & $2.96 \mathrm{E}-05$ & --- & $7.76 \mathrm{E}-05$ & $2.11 E-04$ & $2.33 \mathrm{E}-03$ & $6.34 \mathrm{E}-03$ \\
\hline
\end{tabular}

Assumptions: (a) measured activity used, if available

(b) ${ }^{90} \mathrm{Sr}={ }^{137} \mathrm{Cs} \times 6.00 \mathrm{E}-01$

(c) ${ }^{238} \mathrm{Pu}={ }^{238} \mathrm{Pu} /{ }^{241} \mathrm{Am} \times 1.85 \mathrm{E}-01$

(d) ${ }^{239} \mathrm{Pu}={ }^{239 / 240} \mathrm{Pu} \times 5.05 \mathrm{E}-01$

(e) ${ }^{240} \mathrm{Pu}={ }^{239 / 240} \mathrm{Pu} \times 4.95 \mathrm{E}-01$

(f) ${ }^{241} \mathrm{Pu}={ }^{239 / 240} \mathrm{Pu} \times 2.47 \mathrm{E}+01$

(g) ${ }^{242} \mathrm{Pu}={ }^{239 / 240} \mathrm{Pu} \times 8.28 \mathrm{E}-04$ (h) ${ }^{240} \mathrm{Pu}={ }^{239 / 240} \mathrm{Pu} \times 4.95 \mathrm{E}-01$

(i) ${ }^{241} \mathrm{Pu}={ }^{239 / 240} \mathrm{Pu} \times 2.47 \mathrm{E}+01$

(j) ${ }^{242} \mathrm{Pu}={ }^{239 / 240} \mathrm{Pu} \times 8.28 \mathrm{E}-04$

(k) ${ }^{243} \mathrm{Cm}={ }^{243 / 244} \mathrm{Cm} \times 1.43 \mathrm{E}-02$

(l) ${ }^{244} \mathrm{Cm}={ }^{243 / 244} \mathrm{Cm} \times 9.86 \mathrm{E}-01$

(p) total $=$ removable $\times 3.00 \mathrm{E}+01$ 


\section{REC A-Cell}

\begin{tabular}{|c|c|c|c|c|c|c|}
\hline Sample No. & $47 a$ & $47 \mathrm{~b}$ & $48 a$ & $48 b$ & $49 a$ & $49 b$ \\
\hline $\begin{array}{c}\text { Sample } \\
\text { Location }\end{array}$ & $\begin{array}{c}\text { Electropolish } \\
\text { Skid }\end{array}$ & $\begin{array}{c}\text { Electropolish } \\
\text { Skid }\end{array}$ & $\begin{array}{c}\text { FRG Storage } \\
\text { Rack }\end{array}$ & $\begin{array}{c}\text { FRG Storage } \\
\text { Rack }\end{array}$ & South Wall & South Wall \\
\hline \multicolumn{7}{|c|}{ Smear Gross Count (dpm/100 $\left.\mathrm{cm}^{2}\right)$} \\
\hline Beta/Gamma & --- & -- & -.. & $\cdots$ & 20,000 & 7,500 \\
\hline Alpha & $<20$ & $<20$ & $<20$ & $<20$ & $<20$ & $<20$ \\
\hline \multicolumn{7}{|c|}{ Gamma Exposure Rate (mR/hr) } \\
\hline $2.5 \mathrm{~cm}$ & 0.7 & 0.5 & $<0.5$ & $<0.5$ & $<0.5$ & $<0.5$ \\
\hline $30 \mathrm{~cm}$ & $<0.5$ & $<0.5$ & $<0.5$ & $<0.5$ & $<0.5$ & $<0.5$ \\
\hline \multicolumn{7}{|c|}{ Absorbed Dose Rate (mrad/hr) } \\
\hline $2.5 \mathrm{~cm}$ & 32 & 29 & 6.3 & 3.3 & $<0.5$ & $<0.5$ \\
\hline $30 \mathrm{~cm}$ & $<0.5$ & $<0.5$ & $<0.5$ & $<0.5$ & $<0.5$ & $<0.5$ \\
\hline
\end{tabular}

GEA Measured Isotopic Activitv $\left(\mu \mathrm{Ci} / 100 \mathbf{c m}^{2}\right)$
\begin{tabular}{|c|c|c|c|c|c|c|}
\hline${ }^{54} \mathrm{Mn}$ & $<1 . \mathrm{E}-04$ & $<1 . \mathrm{E}-04$ & $<6 . \mathrm{E}-05$ & $<3 . \mathrm{E}-05$ & $<4 . \mathrm{E}-05$ & $<4 . \mathrm{E}-05$ \\
\hline${ }^{60} \mathrm{Co}$ & $1.25 \mathrm{E}-04$ & $<2 . \mathrm{E}-04$ & $<9 . \mathrm{E}-05$ & $<4 . \mathrm{E}-05$ & $<6 . \mathrm{E}-05$ & $<8 . \mathrm{E}-05$ \\
\hline${ }^{90} \mathrm{Sr}{ }^{(\mathrm{a}, \mathrm{b})}$ & $8.55 \mathrm{E}-02$ & $1.47 \mathrm{E}-01$ & $3.02 \mathrm{E}-02$ & $1.19 \mathrm{E}-02$ & $2.07 \mathrm{E}-03$ & $5.55 \mathrm{E}-04$ \\
\hline${ }^{108 m} \mathrm{Ag}$ & -- & -- & -- & -- & -- & -- \\
\hline${ }^{125} \mathrm{Sb}$ & $<2 . \mathrm{E}-03$ & $<2 . \mathrm{E}-03$ & $<5 . \mathrm{E}-04$ & $<4 . \mathrm{E}-04$ & $<2 . \mathrm{E}-04$ & $<1 . \mathrm{E}-04$ \\
\hline${ }^{134} \mathrm{Cs}$ & $<9 . \mathrm{E}-05$ & $<2 . \mathrm{E}-04$ & $<6 . \mathrm{E}-05$ & $<4 . \mathrm{E}-05$ & $<4 . \mathrm{E}-05$ & $<4 . \mathrm{E}-05$ \\
\hline${ }^{137} \mathrm{Cs}$ & $3.46 \mathrm{E}-01$ & $2.45 \mathrm{E}-01$ & $5.04 \mathrm{E}-02$ & $3.12 \mathrm{E}-02$ & $3.45 \mathrm{E}-03$ & $9.25 \mathrm{E}-04$ \\
\hline${ }^{154} \mathrm{Eu}$ & $<3 . \mathrm{E}-04$ & $<6 . \mathrm{E}-04$ & $<2 . \mathrm{E}-04$ & $<2 . \mathrm{E}-04$ & $<1 . \mathrm{E}-04$ & $<8 . \mathrm{E}-05$ \\
\hline${ }^{155} \mathrm{Eu}$ & $<9 . \mathrm{E}-04$ & $<8 . \mathrm{E}-04$ & $<3 . \mathrm{E}-04$ & $<2 . \mathrm{E}-04$ & $<2 . \mathrm{E}-04$ & $<8 . \mathrm{E}-05$ \\
\hline${ }^{241} \mathrm{Am}$ & $<2 . \mathrm{E}-03$ & $<2 . \mathrm{E}-03$ & $<4 . \mathrm{E}-04$ & $<3 . \mathrm{E}-04$ & $<2 . \mathrm{E}-04$ & $<2 . \mathrm{E}-04$ \\
\hline
\end{tabular}

AEA Measured Isotopic Activity $\left(\mu \mathrm{Ci} / 100 \mathrm{~cm}^{2}\right)$

\begin{tabular}{|c|c|c|c|c|c|c|}
\hline$\left.{ }^{239} \mathrm{Pu}\right|^{240} \mathrm{Pu}$ & $<3 . \mathrm{E}-05$ & $\cdots$ & $\cdots$ & $<2 . \mathrm{E}-06$ & $\cdots$ & $\cdots$ \\
\hline${ }^{238} \mathrm{Pu} /{ }^{241} \mathrm{Am}$ & $1.71 \mathrm{E}-04$ & $\cdots$ & $\cdots$ & $2.07 \mathrm{E}-05$ & $\cdots$ & $\cdots$ \\
\hline${ }^{243} \mathrm{Cm} /{ }^{244} \mathrm{Cm}$ & $8.72 \mathrm{E}-05$ & $\cdots--$ & $\cdots$ & $7.22 \mathrm{E}-06$ & $\cdots$ & $\cdots$ \\
\hline${ }^{242} \mathrm{Cm}$ & $<3 . \mathrm{E}-06$ & $\cdots--$ & $\cdots$ & $<6 . \mathrm{E}-07$ & $\cdots$ & $\cdots$ \\
\hline
\end{tabular}

Measured Gross Activity $\left(\mu \mathrm{Ci} / 100 \mathrm{~cm}^{2}\right)$

\begin{tabular}{|c|c|c|c|c|c|c|}
\hline Alpha & $2.58 \mathrm{E}-04$ & $1.48 \mathrm{E}-03$ & $1.01 \mathrm{E}-04$ & $2.79 \mathrm{E}-05$ & $7.60 \mathrm{E}-06$ & $<2 . \mathrm{E}-05$ \\
\hline Beta/Gamma & $4.32 \mathrm{E}-01$ & $3.92 \mathrm{E}-01$ & $8.06 \mathrm{E}-02$ & $4.31 \mathrm{E}-02$ & $5.52 \mathrm{E}-03$ & $1.48 \mathrm{E}-03$ \\
\hline Total & $4.32 \mathrm{E}-01$ & $3.93 \mathrm{E}-01$ & $8.07 \mathrm{E}-02$ & $4.31 \mathrm{E}-02$ & $5.53 \mathrm{E}-03$ & $1.48 \mathrm{E}-03$ \\
\hline
\end{tabular}

\begin{tabular}{|c|c|c|c|c|c|c|}
\hline${ }^{238} \mathrm{Pu}^{(\mathrm{c})}$ & $3.16 E-05$ & -... & --- & $3.83 E-06$ & --- & $\ldots$ \\
\hline${ }^{239} \mathrm{Pu}^{\text {(d) }}$ & --- & $\cdots$ & $-\cdots$ & -- & $\ldots$ & -- \\
\hline${ }^{240} \mathrm{Pu}^{(\mathrm{e})}$ & $\cdots$ & --- & $\cdots$ & $\cdots$ & $-\cdots$ & $\ldots$ \\
\hline${ }^{241} \mathrm{Pu}^{(1)}$ & $\cdots$ & $\cdots$ & -- & --- & $\cdots$ & $\cdots$ \\
\hline${ }^{242} \mathrm{Pu}^{(\mathrm{g})}$ & --- & $-\cdots$ & $\ldots$ & $\ldots$ & --- & $-\cdots$ \\
\hline${ }^{241} \mathrm{Am}^{(\mathrm{a}, \mathrm{h})}$ & 1.39E-04 & $-\cdots$ & - & $1.69 \mathrm{E}-05$ & -- & $\cdots$ \\
\hline${ }^{243} \mathrm{Cm}^{(\mathrm{l})}$ & $1.25 \mathrm{E}-06$ & $-\cdots$ & $\cdots$ & $1.03 E-07$ & $\ldots$ & --- \\
\hline${ }^{244} \mathrm{Cm}^{(\mathrm{k})}$ & $8.60 \mathrm{E}-05$ & -- & $-\cdots$ & $7.12 \mathrm{E}-06$ & -- & $\cdots$ \\
\hline${ }^{79} \mathrm{Se}^{(\mathrm{m})}$ & $3.29 E-08$ & $\cdots$ & $\cdots$ & $3.98 \mathrm{E}-09$ & $-\cdots$ & $-\cdots$ \\
\hline${ }^{99} \mathrm{Tc}^{(\mathrm{n})}$ & $1.09 \mathrm{E}-06$ & - & $-\cdots$ & $1.32 E-07$ & $\overline{---}$ & $-\cdots$ \\
\hline
\end{tabular}

Assumptions: $\quad$ (a) measured activity used, if available

(b) ${ }^{90} \mathrm{Sr}={ }^{137} \mathrm{Cs} \times 6.00 \mathrm{E}-01$

(c) ${ }^{238} \mathrm{Pu}={ }^{238} \mathrm{Pu} /{ }^{241} \mathrm{Am} \times 1.85 \mathrm{E}-01$

(d) ${ }^{239} \mathrm{Pu}={ }^{239 / 240} \mathrm{Pu} \times 5.05 \mathrm{E}-01$

(e) ${ }^{240} \mathrm{Pu}={ }^{239 / 240} \mathrm{Pu} \times 4.95 \mathrm{E}-01$

(f) ${ }^{241} \mathrm{Pu}={ }^{239 / 240} \mathrm{Pu} \times 2.47 \mathrm{E}+01$

(g) ${ }^{242} \mathrm{Pu}={ }^{239 / 240} \mathrm{Pu} \times 8.28 \mathrm{E}-04$ (h) ${ }^{241} \mathrm{Am}={ }^{238} \mathrm{Pu} /{ }^{241} \mathrm{Am} \times 8.15 \mathrm{E}-01$

(j) ${ }^{243} \mathrm{Cm}={ }^{243 / 244} \mathrm{Cm} \times 1.43 \mathrm{E}-02$

(k) ${ }^{244} \mathrm{Cm}={ }^{243 / 244} \mathrm{Cm} \times 9.86 \mathrm{E}-01$

(m) ${ }^{79} \mathrm{Se}={ }^{241} \mathrm{Am} \times 2.36 \mathrm{E}-04$

(n) ${ }^{99} \mathrm{Tc}={ }^{241} \mathrm{Am} \times 7.83 \mathrm{E}-03$

(p) total $=$ removable $\times 3.00 \mathrm{E}+01$ 


\section{REC A-Cell}

\begin{tabular}{|c|c|c|c|c|}
\hline Sample No. & \multicolumn{3}{|c|}{ Estimated Isotopic Activity $\left(\mu \mathrm{Ci} / 100 \mathrm{~cm}^{2}\right)^{(\mathrm{p})}$} \\
\hline \multirow{2}{*}{$\begin{array}{c}\text { Sample } \\
\text { Location }\end{array}$} & \multicolumn{2}{|c|}{ Removable } & \multicolumn{2}{|c|}{ Total } \\
\cline { 2 - 5 } & Average & Max & Average & Max \\
\hline
\end{tabular}

Smear Gross Count (dpm/100 $\left.\mathrm{cm}^{2}\right)$

\begin{tabular}{|c|c|c|c|c|}
\hline Beta/Gamma & NA & NA & $\mathrm{NA}$ & NA \\
\hline Alpha & NA & NA & NA & NA \\
\hline \multicolumn{5}{|c|}{ Gamma Exposure Rate (mR/hr) } \\
\hline $2.5 \mathrm{~cm}$ & NA & NA & NA & NA \\
\hline $30 \mathrm{~cm}$ & NA & $\overline{N A}$ & $\overline{N A}$ & $\overline{N A}$ \\
\hline \multicolumn{5}{|c|}{ Absorbed Dose Rate (mrad/hr) } \\
\hline $2.5 \mathrm{~cm}$ & NA & NA & $\mathrm{NA}$ & NA \\
\hline $30 \mathrm{~cm}$ & NA & NA & $\mathrm{NA}$ & $\mathrm{NA}$ \\
\hline \multicolumn{5}{|c|}{ GEA Measured Isotopic Activity $\left(\mu \mathrm{Ci} / 100 \mathrm{~cm}^{2}\right)$} \\
\hline${ }^{54} \mathrm{Mn}$ & ND & ND & ND & ND \\
\hline${ }^{60} \mathrm{Co}$ & $1.25 E-04$ & $1.25 \mathrm{E}-04$ & 3.75E-03 & $3.75 E-03$ \\
\hline${ }^{90} \mathrm{Sr}^{(\mathrm{a}, \mathrm{b})}$ & $4.62 E-02$ & 1.47E-01 & $1.39 E+00$ & $4.41 E+00$ \\
\hline${ }^{108 \mathrm{~m}} \mathrm{Ag}$ & $\cdots$ & $\cdots$ & $\cdots$ & $\cdots$ \\
\hline${ }^{125} \mathrm{Sb}$ & ND & ND & ND & ND \\
\hline${ }^{134} \mathrm{Cs}$ & ND & ND & $\mathrm{ND}$ & ND \\
\hline${ }^{137} \mathrm{Cs}$ & $1.13 E-01$ & $3.46 \mathrm{E}-01$ & $3.38 \mathrm{E}+00$ & $1.04 \mathrm{E}+01$ \\
\hline${ }^{154} \mathrm{Eu}$ & ND & ND & ND & ND \\
\hline${ }^{155} \mathrm{Eu}$ & ND & ND & $\mathrm{ND}$ & $\overline{N D}$ \\
\hline${ }^{241} \mathrm{Am}$ & ND & ND & ND & ND \\
\hline
\end{tabular}

AEA Measured Isotopic Activity $\left(\mu \mathrm{Ci} / 100 \mathrm{~cm}^{2}\right)$

\begin{tabular}{|c|c|c|c|c|}
\hline${ }^{239} \mathrm{Pu} /{ }^{240} \mathrm{Pu}$ & ND & ND & ND & ND \\
\hline${ }^{238} \mathrm{Pu} /{ }^{241} \mathrm{Am}$ & $9.59 \mathrm{E}-05$ & $1.71 \mathrm{E}-04$ & $2.88 \mathrm{E}-03$ & $5.13 \mathrm{E}-03$ \\
\hline${ }^{243} \mathrm{Cm} /{ }^{244} \mathrm{Cm}$ & $4.72 \mathrm{E}-05$ & $8.72 \mathrm{E}-05$ & $1.42 \mathrm{E}-03$ & $2.62 \mathrm{E}-03$ \\
\hline${ }^{242} \mathrm{Cm}$ & ND & ND & ND & ND \\
\hline \multicolumn{5}{|c|}{ Measured Gross Activitv $\left(\mu \mathrm{Ci} / 100 \mathrm{~cm}^{2}\right)$} \\
\hline Alpha & $3.75 \mathrm{E}-04$ & $1.48 \mathrm{E}-03$ & $1.12 \mathrm{E}-02$ & $4.44 \mathrm{E}-02$ \\
\hline Beta/Gamma & $1.59 \mathrm{E}-01$ & $4.32 \mathrm{E}-01$ & $4.77 \mathrm{E}+00$ & $1.29 \mathrm{E}+01$ \\
\hline Total & $1.59 \mathrm{E}-01$ & $4.32 \mathrm{E}-01$ & $4.78 \mathrm{E}+00$ & $1.30 \mathrm{E}+01$ \\
\hline
\end{tabular}

Calculated Isotopic Activitv $\left(\mu \mathrm{Ci} / 100 \mathrm{~cm}^{2}\right)$

\begin{tabular}{|c|c|c|c|c|}
\hline${ }^{238} \mathrm{Pu}^{(\mathrm{c})}$ & $1.77 \mathrm{E}-05$ & $3.16 \mathrm{E}-05$ & $5.32 \mathrm{E}-04$ & $9.49 \mathrm{E}-04$ \\
\hline${ }^{239} \mathrm{Pu}^{(\mathrm{d})}$ & ND & ND & ND & ND \\
\hline${ }^{240} \mathrm{Pu}^{(\mathrm{e})}$ & ND & ND & ND & ND \\
\hline${ }^{241} \mathrm{Pu}^{(\mathrm{f})}$ & $\mathrm{ND}$ & ND & ND & $\mathrm{ND}$ \\
\hline${ }^{242} \mathrm{Pu}^{(\mathrm{g})}$ & ND & ND & ND & ND \\
\hline${ }^{241} \mathrm{Am}^{(\mathrm{a}, \mathrm{h})}$ & $7.81 \mathrm{E}-05$ & $1.39 \mathrm{E}-04$ & $2.34 \mathrm{E}-03$ & $4.18 \mathrm{E}-03$ \\
\hline${ }^{243} \mathrm{Cm}^{(0)}$ & $6.75 \mathrm{E}-07$ & $1.25 E-06$ & $2.03 E-05$ & 3.74E-05 \\
\hline${ }^{244} \mathrm{Cm}^{(\mathrm{k})}$ & $4.65 \mathrm{E}-05$ & $8.60 \mathrm{E}-05$ & $1.40 \mathrm{E}-03$ & $2.58 \mathrm{E}-03$ \\
\hline${ }^{79} \mathrm{Se}^{(m)}$ & 1.84E-08 & $3.29 E-08$ & $5.53 E-07$ & 9.87E-07 \\
\hline${ }^{99} \mathrm{Tc}^{(\mathrm{n})}$ & $6.12 \mathrm{E}-07$ & $1.09 \mathrm{E}-06$ & 1.83E-05 & $3.27 E-05$ \\
\hline
\end{tabular}

Assumptions: $\quad$ (a) measured activity used, if available

(b) ${ }^{90} \mathrm{Sr}={ }^{137} \mathrm{Cs} \times 6.00 \mathrm{E}-01$

(c) ${ }^{238} \mathrm{Pu}={ }^{238} \mathrm{Pu} /{ }^{241} \mathrm{Am} \times 1.85 \mathrm{E}-01$
(d) ${ }^{239} \mathrm{Pu}={ }^{239 / 240} \mathrm{Pu} \times 5.05 \mathrm{E}-01$
(e) ${ }^{240} \mathrm{Pu}={ }^{239 / 240} \mathrm{Pu} \times 4.95 \mathrm{E}-01$
(f) ${ }^{241} \mathrm{Pu}={ }^{239 / 240} \mathrm{Pu} \times 2.47 \mathrm{E}+01$
(g) ${ }^{242} \mathrm{Pu}={ }^{239 / 240} \mathrm{Pu} \times 8.28 \mathrm{E}-04$

(h) ${ }^{241} \mathrm{Am}={ }^{238} \mathrm{Pu} /{ }^{241} \mathrm{Am} \times 8.15 \mathrm{E}-01$

(j) ${ }^{243} \mathrm{Cm}={ }^{243 / 244} \mathrm{Cm} \times 1.43 \mathrm{E}-02$

(k) ${ }^{244} \mathrm{Cm}={ }^{243 / 244} \mathrm{Cm} \times 9.86 \mathrm{E}-01$

(m) ${ }^{79} \mathrm{Se}={ }^{241} \mathrm{Am} \times 2.36 \mathrm{E}-04$

(n) ${ }^{99} \mathrm{Tc}={ }^{241} \mathrm{Am} \times 7.83 \mathrm{E}-03$

(p) total $=$ removable $\times 3.00 \mathrm{E}+01$ 


\section{REC B-Cell}

\begin{tabular}{|c|c|c|c|c|c|c|c|c|}
\hline Sample No. & $39 a$ & $39 b$ & $40 \mathbf{a}$ & $40 \mathrm{~b}$ & 42a & $42 b$ & $43 \mathbf{a}$ & $43 b$ \\
\hline $\begin{array}{c}\text { Sample } \\
\text { Location }\end{array}$ & $\begin{array}{c}\text { S Wall } n \\
\text { side }\end{array}$ & $\begin{array}{c}\text { S Wall } \mathrm{n} \\
\text { side }\end{array}$ & $\begin{array}{c}\text { N side } \\
\text { fuel }\end{array}$ & $\begin{array}{c}N \text { side } \\
\text { fuel }\end{array}$ & $\begin{array}{l}\text { S Wall } \\
\text { center }\end{array}$ & $\begin{array}{l}\text { S Wall } \\
\text { center }\end{array}$ & $\begin{array}{c}\mathrm{N} \text { fuel } \\
\text { storage }\end{array}$ & $\begin{array}{c}\mathrm{N} \text { fuel } \\
\text { storage }\end{array}$ \\
\hline \multicolumn{9}{|c|}{ Smear Gross Count (dpm/100 cm $\left.{ }^{2}\right)$} \\
\hline Beta/Gamma & \begin{tabular}{|c|}
-- \\
\end{tabular} & $-\cdots$ & --- & --- & --- & -- & $-\ldots$ & --- \\
\hline Alpha & 49,000 & 52,500 & 17,500 & 17,500 & 420,000 & 560,000 & 245,000 & 28,000 \\
\hline \multicolumn{9}{|c|}{ Gamma Exposure Rate (mR/hr) } \\
\hline $2.5 \mathrm{~cm}$ & 300 & 225 & 12 & 14 & 120 & 300 & 12 & 30 \\
\hline $30 \mathrm{~cm}$ & 10 & 8 & 2.5 & 1.5 & 4 & 10 & 3 & 3.5 \\
\hline \multicolumn{9}{|c|}{ Absorbed Dose Rate (mrad/hr) } \\
\hline $2.5 \mathrm{~cm}$ & 23,700 & 19,175 & 738 & 737 & 8,880 & 26,700 & 438 & 3,270 \\
\hline $30 \mathrm{~cm}$ & 580 & 434 & 19 & 21 & 202 & 490 & 18 & 59 \\
\hline \multicolumn{9}{|c|}{ GEA Measured Isotopic Activity $\left(\mu \mathrm{Ci} / 100 \mathrm{~cm}^{2}\right)$} \\
\hline${ }^{54} \mathrm{Mn}$ & $<3 . \mathrm{E}-02$ & $<4 . E-02$ & $<3 . E-03$ & $<3 . E-03$ & $<5 . E-03$ & $<2 . \mathrm{E}-02$ & $<2 . E-03$ & $<4 . \mathrm{E}-03$ \\
\hline${ }^{60} \mathrm{Co}$ & $6.50 \mathrm{E}-02$ & $<2 . E-02$ & $1.02 \mathrm{E}-02$ & $<5 . E-03$ & $<6 . \mathrm{E}-03$ & $<2 . \mathrm{E}-02$ & $<4 . E-03$ & $<5 . \mathrm{E}-03$ \\
\hline${ }^{90} \mathrm{Sr}^{(\mathrm{a}, \mathrm{b})}$ & $1.43 \mathrm{E}+02$ & $7.32 \mathrm{E}+01$ & $4.62 \mathrm{E}+00$ & $3.84 \mathrm{E}+00$ & $4.30 E+01$ & $7.68 \mathrm{E}+01$ & $2.57 E+00$ & 1.37E+01 \\
\hline${ }^{108 m} \mathrm{Ag}$ & --- & $--\cdot$ & --- & -.- & --- & $\cdots$ & -- & $\cdots$ \\
\hline${ }^{125} \mathrm{Sb}$ & $<5 . E-01$ & $<4 . E-01$ & $<7 . E-02$ & $<7 . E-02$ & $<2 . E-01$ & $<4 . E-01$ & $<3 . E-02$ & $<2 . E-01$ \\
\hline${ }^{134} \mathrm{Cs}$ & 1.09E-01 & $<4 . E-02$ & $<4 . \mathrm{E}-03$ & $<4 . E-03$ & $<8 . E-03$ & $<2 . E-02$ & $<2 . \mathrm{E}-03$ & $<6 . E-03$ \\
\hline${ }^{137} \mathrm{Cs}$ & $2.39 E+02$ & $1.68 \mathrm{E}+02$ & $7.70 \mathrm{E}+00$ & $7.16 \mathrm{E}+00$ & $4.30 \mathrm{E}+01$ & $1.28 \mathrm{E}+02$ & $1.62 \mathrm{E}+00$ & $2.29 \mathrm{E}+01$ \\
\hline${ }^{154} \mathrm{Eu}$ & $6.26 \mathrm{E}-02$ & $2.87 \mathrm{E}-01$ & $2.07 \mathrm{E}-02$ & $1.73 \mathrm{E}-02$ & $1.67 E-01$ & $5.25 \mathrm{E}-01$ & $<6 . \mathrm{E}-03$ & $5.33 \mathrm{E}-02$ \\
\hline${ }^{155} \mathrm{Eu}$ & $<3 . E-01$ & $<2 . \mathrm{E}-01$ & $<3 . \mathrm{E}-02$ & $<4 . E-02$ & $<9 . \mathrm{E}-02$ & $<2 . E-01$ & $<2 . \mathrm{E}-02$ & $<6 . E-02$ \\
\hline${ }^{241} \mathrm{Am}$ & $<7 . E-01$ & $<4 . E-01$ & $<4 . \mathrm{E}-02$ & $<6 . E-02$ & $1.86 \mathrm{E}-01$ & $6.49 \mathrm{E}-01$ & $<3 . E-02$ & $<8 . E-02$ \\
\hline \multicolumn{9}{|c|}{ AEA Measured Isotopic Activity $\left(\mu \mathrm{Ci} / 100 \mathrm{~cm}^{2}\right)$} \\
\hline${ }^{239} \mathrm{Pu} /{ }^{240} \mathrm{Pu}$ & $\cdots$ & $1.79 E-02$ & $-\cdots$ & $1.80 \mathrm{E}-03$ & $<2 . E-02$ & $-\cdots$ & $2.15 \mathrm{E}-03$ & -.. \\
\hline${ }^{238} \mathrm{Pu} /{ }^{241} \mathrm{Am}$ & $-\cdots$ & 1.07E-01 & $-\cdots$ & $7.50 \mathrm{E}-03$ & $2.54 \mathrm{E}-01$ & $\cdots$ & $5.64 E-03$ & $\cdots$ \\
\hline${ }^{243} \mathrm{Cm} /{ }^{244} \mathrm{Cm}$ & -- & $5.34 \mathrm{E}-02$ & -- & $2.69 \mathrm{E}-03$ & $8.23 \mathrm{E}-02$ & -- & $2.11 E-03$ & -- \\
\hline${ }^{242} \mathrm{Cm}$ & $\cdots$ & $<9 . \mathrm{E}-05$ & $-\cdots$ & $<2 . E-04$ & $<1 . \mathrm{E}-03$ & $-\cdots$ & $<5 . E-05$ & $\cdots$ \\
\hline \multicolumn{9}{|c|}{ Measured Gross Activity $\left(\mu \mathrm{Ci} / 100 \mathrm{~cm}^{2}\right)$} \\
\hline Alpha & $4.26 \mathrm{E}-01$ & $1.78 \mathrm{E}-01$ & $1.15 \mathrm{E}-02$ & $1.20 \mathrm{E}-02$ & $3.36 \mathrm{E}-01$ & $1.16 \mathrm{E}+00$ & $9.90 \mathrm{E}-03$ & $4.55 \mathrm{E}-02$ \\
\hline Beta/Gamma & $3.83 E+02$ & $2.41 \mathrm{E}+02$ & $1.24 \mathrm{E}+01$ & $1.10 \mathrm{E}+01$ & $8.62 E+01$ & $2.05 \mathrm{E}+02$ & $4.19 \mathrm{E}+00$ & $3.67 E+01$ \\
\hline Total & $3.83 E+02$ & $2.42 E+02$ & $1.24 \mathrm{E}+01$ & $1.10 \mathrm{E}+01$ & $8.65 E+01$ & $2.06 \mathrm{E}+02$ & $4.20 E+00$ & $3.67 E+01$ \\
\hline \multicolumn{9}{|c|}{ Calculated Isotopic Activity $\left(\mu \mathrm{Ci} / 100 \mathrm{~cm}^{2}\right)$} \\
\hline${ }^{238} \mathrm{Pu}^{(\mathrm{c})}$ & -- & $1.98 \mathrm{E}-02$ & -- & $1.39 \mathrm{E}-03$ & $4.70 \mathrm{E}-02$ & --- & $1.04 \mathrm{E}-03$ & $\cdots$ \\
\hline${ }^{239} \mathrm{Pu}^{(\mathrm{d})}$ & $-\cdots$ & $9.04 \mathrm{E}-03$ & $\cdots$ & $9.09 E-04$ & -- & $-\cdots$ & $1.09 \mathrm{E}-03$ & $-\cdots$ \\
\hline${ }^{240} \mathrm{Pu}^{(\mathrm{e})}$ & $-\cdots$ & $8.86 \mathrm{E}-03$ & -- & 8.91E-04 & $\cdots$ & --- & $1.06 \mathrm{E}-03$ & -- \\
\hline${ }^{241} \mathrm{Pu}^{(f)}$ & $-\cdots$ & $4.42 \mathrm{E}-01$ & $\cdots$ & $4.45 \mathrm{E}-02$ & -- & $-\cdots$ & $5.31 \mathrm{E}-02$ & $\cdots$ \\
\hline${ }^{242} \mathrm{Pu}^{(\mathrm{g})}$ & $-\cdots$ & $1.48 \mathrm{E}-05$ & --- & 1.49E-06 & $\cdots$ & $-\cdots$ & $1.78 \mathrm{E}-06$ & -- \\
\hline${ }^{241} \mathrm{Am}^{(\mathrm{a}, \mathrm{h})}$ & $-\cdots$ & $8.72 E-02$ & $\cdots$ & $6.11 \mathrm{E}-03$ & $1.86 \mathrm{E}-01$ & $6.49 E-01$ & $4.60 \mathrm{E}-03$ & $\cdots$ \\
\hline${ }^{243} \mathrm{Cm}^{(j)}$ & $\cdots$ & $7.64 \mathrm{E}-04$ & --- & $3.85 E-05$ & $1.18 \mathrm{E}-03$ & $\cdots$ & $3.02 E-05$ & $-\cdots$ \\
\hline${ }^{244} \mathrm{Cm}^{(\mathrm{k})}$ & -- & $5.26 \mathrm{E}-02$ & $-\cdots$ & 2.65E-03 & $8.11 \mathrm{E}-02$ & -- & $2.08 \mathrm{E}-03$ & $-\cdots$ \\
\hline${ }^{79} \mathrm{Se}^{(\mathrm{m})}$ & $\cdots$ & $2.06 E-05$ & $\cdots$ & $1.44 \mathrm{E}-06$ & 4.39E-05 & $1.53 \mathrm{E}-04$ & $1.08 \mathrm{E}-06$ & $\ldots$ \\
\hline${ }^{99} \mathrm{Tc}^{(\mathrm{n})}$ & -- & $6.83 E-04$ & --- & $4.79 E-05$ & $1.46 \mathrm{E}-03$ & $5.08 \mathrm{E}-03$ & $3.60 \mathrm{E}-05$ & $\cdots$ \\
\hline
\end{tabular}

\section{Assumptions:}

(a) measured activity used, if available

(b) ${ }^{90} \mathrm{Sr}={ }^{137} \mathrm{Cs} \times 6.00 \mathrm{E}-01$

(c) ${ }^{238} \mathrm{Pu}={ }^{238} \mathrm{Pu} /{ }^{241} \mathrm{Am} \times 1.85 \mathrm{E}-01$

(d) ${ }^{239} \mathrm{Pu}={ }^{239 / 240} \mathrm{Pu} \times 5.05 \mathrm{E}-01$

(e) ${ }^{240} \mathrm{Pu}={ }^{239 / 240} \mathrm{Pu} \times 4.95 \mathrm{E}-01$

(f) ${ }^{241} \mathrm{Pu}={ }^{239 / 240} \mathrm{Pu} \times 2.47 \mathrm{E}+01$

(g) ${ }^{242} \mathrm{Pu}={ }^{239 / 240} \mathrm{Pu} \times 8.28 \mathrm{E}-04$ (h) ${ }^{241} \mathrm{Am}={ }^{238} \mathrm{Pu} /{ }^{241} \mathrm{Am} \times 8.15 \mathrm{E}-01$

(j) ${ }^{243} \mathrm{Cm}={ }^{243 / 244} \mathrm{Cm} \times 1.43 \mathrm{E}-02$

(k) ${ }^{244} \mathrm{Cm}={ }^{243 / 244} \mathrm{Cm} \times 9.86 \mathrm{E}-01$

(m) ${ }^{79} \mathrm{Se}={ }^{241} \mathrm{Am} \times 2.36 \mathrm{E}-04$

(n) ${ }^{99} \mathrm{Tc}={ }^{241} \mathrm{Am} \times 7.83 \mathrm{E}-03$

(p) total $=$ removable $\times 3.00 \mathrm{E}+01$ 


\section{REC B-Cell}

\begin{tabular}{|c|c|c|c|c|c|c|c|}
\hline Sample No. & $44 a$ & $44 b$ & $45 a$ & $45 b$ & \multicolumn{2}{|c|}{ Estimated Isotopic Activity $\left(\mu \mathrm{Ci} / 100 \mathrm{~cm}^{2}\right)^{(\mathrm{p})}$} \\
\hline $\begin{array}{c}\text { Sample } \\
\text { Location }\end{array}$ & $\begin{array}{c}\text { S fuel } \\
\text { storage }\end{array}$ & $\begin{array}{c}\text { S fuel } \\
\text { storage }\end{array}$ & $\begin{array}{c}\text { W Wall } \\
\text { center }\end{array}$ & $\begin{array}{c}\text { W Wall } \\
\text { center }\end{array}$ & \multicolumn{2}{|c|}{ Removable } & \multicolumn{2}{|c|}{ Total } \\
\cline { 5 - 7 } & & & & Average & Max & Average & Max \\
\hline
\end{tabular}

\begin{tabular}{|c|c|c|c|c|c|c|c|c|}
\hline \multicolumn{9}{|c|}{ Smear Gross Count (dpm/100 cm $\left.{ }^{2}\right)$} \\
\hline Beta/Gamma & $\cdots$ & --- & $\cdots$ & $-\cdot$ & NA & NA & NA & NA \\
\hline Alpha & 42,000 & 21,000 & 140,000 & 210,000 & NA & NA & NA & NA \\
\hline \multicolumn{9}{|c|}{ Gamma Exposure Rate (mR/hr) } \\
\hline $2.5 \mathrm{~cm}$ & 23 & 10 & 4 & 7 & NA & NA & $\mathrm{NA}$ & $\mathrm{NA}$ \\
\hline $30 \mathrm{~cm}$ & 3.5 & 3 & 6 & 10 & $\mathrm{NA}$ & NA & NA & NA \\
\hline \multicolumn{9}{|c|}{ Absorbed Dose Rate (mrad/hr) } \\
\hline $2.5 \mathrm{~cm}$ & 2,378 & 439 & 58 & 227 & NA & NA & $\mathrm{NA}$ & NA \\
\hline $30 \mathrm{~cm}$ & 38 & 18 & 168 & 670 & NA & NA & $\mathrm{NA}$ & $\overline{N A}$ \\
\hline
\end{tabular}

GEA Measured Isotopic Activity $\left(\mu \mathrm{Ci} / 100 \mathrm{~cm}^{2}\right)$

\begin{tabular}{|c|c|c|c|c|c|c|c|c|}
\hline & & & & & & & & \\
\hline${ }^{54} \mathrm{Mn}$ & $<3 . E-03$ & $<2 . E-03$ & $<6 . E-03$ & $<3 . \mathrm{E}-02$ & ND & ND & ND & ND \\
\hline${ }^{60} \mathrm{Co}$ & $<4 . E-03$ & $<4 . E-03$ & $<1 . E-02$ & $<1 . \mathrm{E}-02$ & $3.76 \mathrm{E}-02$ & $6.50 \mathrm{E}-02$ & $1.13 E+00$ & $1.95 E+00$ \\
\hline${ }^{90} \mathrm{Sr}^{(\mathrm{a}, \mathrm{b})}$ & $1.51 \mathrm{E}+01$ & $1.89 E+00$ & $2.89 E+01$ & $1.97 E+02$ & $5.04 E+01$ & $1.97 E+02$ & $1.51 E+03$ & $5.92 E+03$ \\
\hline${ }^{108 \mathrm{~m}} \mathrm{Aq}$ & -- & --- & $\cdots$ & $\cdots$ & $-\cdots$ & $\ldots$ & -- & $\ldots$ \\
\hline${ }^{125} \mathrm{Sb}$ & $<2 . E-01$ & $<4 . E-02$ & $<3 . E-01$ & $<6$.E-01 & ND & ND & ND & ND \\
\hline${ }^{134} \mathrm{Cs}$ & $<6 . \mathrm{E}-03$ & $<3 . \mathrm{E}-03$ & $<7 . E-03$ & $<3 . E-02$ & $1.09 \mathrm{E}-01$ & $1.09 \mathrm{E}-01$ & $3.27 E+00$ & $3.27 E+00$ \\
\hline${ }^{137} \mathrm{Cs}$ & $2.51 E+01$ & $4.90 E+00$ & $5.34 E+01$ & $3.29 E+02$ & $8.58 E+01$ & $3.29 E+02$ & $2.57 E+03$ & $9.87 E+03$ \\
\hline${ }^{154} \mathrm{Eu}$ & $6.59 \mathrm{E}-02$ & $1.16 \mathrm{E}-02$ & $8.41 \mathrm{E}-02$ & $1.71 \mathrm{E}-01$ & 1.33E-01 & $5.25 \mathrm{E}-01$ & $4.00 \mathrm{E}+00$ & $1.58 \mathrm{E}+01$ \\
\hline${ }^{155} \mathrm{Eu}$ & $<6 . \mathrm{E}-02$ & $<2 . E-02$ & $<9 . \mathrm{E}-02$ & $<3 . E-01$ & ND & ND & ND & ND \\
\hline${ }^{241} \mathrm{Am}$ & $<7 . E-02$ & $<4 . E-02$ & $<2 . E-01$ & 2.77E-01 & $3.71 \mathrm{E}-01$ & $6.49 \mathrm{E}-01$ & $1.11 \mathrm{E}+01$ & $1.95 \mathrm{E}+01$ \\
\hline \multicolumn{9}{|c|}{ AEA Measured Isotopic Activity $\left(\mu \mathrm{Ci} / 100 \mathrm{~cm}^{2}\right)$} \\
\hline${ }^{239} \mathrm{Pu} /{ }^{240} \mathrm{Pu}$ & $\cdots$ & $4.80 \mathrm{E}-03$ & $8.47 \mathrm{E}-03$ & -- & $7.02 E-03$ & $1.79 \mathrm{E}-02$ & $2.11 \mathrm{E}-01$ & $5.37 \mathrm{E}-01$ \\
\hline${ }^{238} \mathrm{Pu} /{ }^{241} \mathrm{Am}$ & $-\cdots$ & $1.46 \mathrm{E}-02$ & $7.64 \mathrm{E}-02$ & $-\cdots$ & $7.75 \mathrm{E}-02$ & $2.54 \mathrm{E}-01$ & $2.33 \mathrm{E}+00$ & $7.62 E+00$ \\
\hline${ }^{243} \mathrm{Cm} /{ }^{244} \mathrm{Cm}$ & $\cdots$ & $3.47 \mathrm{E}-03$ & $2.41 \mathrm{E}-02$ & $\cdots$ & $2.80 \mathrm{E}-02$ & $8.23 \mathrm{E}-02$ & $8.40 \mathrm{E}-01$ & $2.47 E+00$ \\
\hline${ }^{242} \mathrm{Cm}$ & $-\cdots$ & $<1 . \mathrm{E}-04$ & $<5 . \mathrm{E}-04$ & --- & ND & ND & ND & ND \\
\hline \multicolumn{5}{|c|}{ Measured Gross Activity $(\mu \mathrm{Ci} / 100$} & --- & --- & --- & $-\cdots$ \\
\hline Alpha & 4.10E-02 & $2.29 \mathrm{E}-02$ & $1.09 \mathrm{E}-01$ & $3.69 \mathrm{E}-01$ & 2.27E-01 & $1.16 \mathrm{E}+00$ & $6.80 \mathrm{E}+00$ & $3.48 E+01$ \\
\hline Beta/Gamma & $4.02 E+01$ & $6.80 \mathrm{E}+00$ & $8.24 \mathrm{E}+01$ & $5.27 E+02$ & $1.36 \mathrm{E}+02$ & $5.27 E+02$ & $4.09 E+03$ & $1.58 E+04$ \\
\hline Total & $4.03 E+01$ & $6.82 E+00$ & $8.25 E+01$ & $5.27 \mathrm{E}+02$ & $1.37 \mathrm{E}+02$ & $5.27 E+02$ & $4.10 \mathrm{E}+03$ & $1.58 \mathrm{E}+04$ \\
\hline \multicolumn{9}{|c|}{ Calculated Isotopic Activity $\left(\mu \mathrm{Ci} / 100 \mathrm{~cm}^{2}\right)$} \\
\hline${ }^{238} \mathrm{Pu}^{(\mathrm{c})}$ & 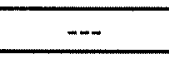 & $2.70 \mathrm{E}-03$ & $1.41 \mathrm{E}-02$ & -- & $1.43 E-02$ & $4.70 \mathrm{E}-02$ & $4.30 \mathrm{E}-01$ & $1.41 \mathrm{E}+00$ \\
\hline${ }^{239} \mathrm{Pu}^{\text {(d) }}$ & -- & $2.42 \mathrm{E}-03$ & $4.28 \mathrm{E}-03$ & $\ldots$ & $3.55 \mathrm{E}-03$ & $9.04 \mathrm{E}-03$ & $1.06 \mathrm{E}-01$ & $2.71 E-01$ \\
\hline${ }^{240} \mathrm{Pu}^{(\mathrm{e})}$ & $\cdots$ & $2.38 \mathrm{E}-03$ & $4.19 \mathrm{E}-03$ & $\cdots$ & $3.48 E-03$ & $8.86 \mathrm{E}-03$ & $1.04 \mathrm{E}-01$ & $2.66 \mathrm{E}-01$ \\
\hline${ }^{241} \mathrm{Pu}^{(f)}$ & -- & 1.19E-01 & $2.09 \mathrm{E}-01$ & $\cdots$ & $1.73 \mathrm{E}-01$ & 4.42E-01 & $5.20 \mathrm{E}+00$ & $1.33 E+01$ \\
\hline${ }^{242} \mathrm{Pu}^{(\mathrm{g})}$ & --- & $3.97 \mathrm{E}-06$ & $7.01 E-06$ & $\cdots$ & $5.82 E-06$ & $1.48 \mathrm{E}-05$ & $1.74 \mathrm{E}-04$ & $4.45 E-04$ \\
\hline${ }^{241} \mathrm{Am}^{(\mathrm{a}, \mathrm{h})}$ & $\cdots$ & $1.19 E-02$ & $6.23 E-02$ & $2.77 \mathrm{E}-01$ & $1.61 \mathrm{E}-01$ & $6.49 \mathrm{E}-01$ & $4.82 E+00$ & $1.95 E+01$ \\
\hline${ }^{243} \mathrm{Cm}^{(0)}$ & --- & $4.96 \mathrm{E}-05$ & $3.45 E-04$ & -- & $4.01 E-04$ & $1.18 E-03$ & $1.20 \mathrm{E}-02$ & $3.53 E-02$ \\
\hline${ }^{244} \mathrm{Cm}^{(\mathrm{k})}$ & $-\cdots$ & $3.42 \mathrm{E}-03$ & $2.38 E-02$ & --- & $2.76 \mathrm{E}-02$ & $8.11 \mathrm{E}-02$ & $8.28 E-01$ & $2.43 E+00$ \\
\hline${ }^{79} \mathrm{Se}^{(\mathrm{m})}$ & $\cdots$ & $2.81 E-06$ & $1.47 \mathrm{E}-05$ & $6.54 \mathrm{E}-05$ & $3.79 E-05$ & $1.53 \mathrm{E}-04$ & $1.14 \mathrm{E}-03$ & $4.59 \mathrm{E}-03$ \\
\hline${ }^{99} \mathrm{Tc}^{(\mathrm{n})}$ & -- & $9.32 E-05$ & $4.88 \mathrm{E}-04$ & $2.17 E-03$ & $1.26 \mathrm{E}-03$ & $5.08 E-03$ & $3.77 \mathrm{E}-02$ & $1.52 \mathrm{E}-01$ \\
\hline
\end{tabular}

\section{Assumptions:}

(a) measured activity used, if available

(b) ${ }^{90} \mathrm{Sr}={ }^{137} \mathrm{Cs} \times 6.00 \mathrm{E}-01$

(c) ${ }^{238} \mathrm{Pu}={ }^{238} \mathrm{Pu} /{ }^{241} \mathrm{Am} \times 1.85 \mathrm{E}-01$

(d) ${ }^{239} \mathrm{Pu}={ }^{239 / 240} \mathrm{Pu} \times 5.05 \mathrm{E}-01$

(e) ${ }^{240} \mathrm{Pu}={ }^{239 / 240} \mathrm{Pu} \times 4.95 \mathrm{E}-01$

(f) ${ }^{241} \mathrm{Pu}={ }^{239 / 240} \mathrm{Pu} \times 2.47 \mathrm{E}+01$

(g) ${ }^{242} \mathrm{Pu}={ }^{239 / 240} \mathrm{Pu} \times 8.28 \mathrm{E}-04$ (h) ${ }^{241} \mathrm{Am}={ }^{238} \mathrm{Pu} /{ }^{241} \mathrm{Am} \times 8.15 \mathrm{E}-01$

(j) ${ }^{243} \mathrm{Cm}={ }^{243 / 244} \mathrm{Cm} \times 1.43 \mathrm{E}-02$

(k) ${ }^{244} \mathrm{Cm}={ }^{243 / 244} \mathrm{Cm} \times 9.86 \mathrm{E}-01$

(m) ${ }^{79} \mathrm{Se}={ }^{241} \mathrm{Am} \times 2.36 \mathrm{E}-04$

(n) ${ }^{99} \mathrm{Tc}={ }^{241} \mathrm{Am} \times 7.83 \mathrm{E}-03$

(p) total $=$ removable $\times 3.00 E+01$ 


\section{REC C-Cell}

\begin{tabular}{|c|c|c|c|c|c|c|c|}
\hline Sample No. & $18 \mathbf{a}$ & $18 b$ & $19 a$ & $19 b$ & $20 \mathrm{a}$ & $20 b$ & $21 \mathbf{a}$ \\
\hline $\begin{array}{c}\text { Sample } \\
\text { Location }\end{array}$ & $\begin{array}{c}\text { Sump } \\
\text { trench S }\end{array}$ & $\begin{array}{c}\text { Sump } \\
\text { trench } \mathrm{S}\end{array}$ & $\begin{array}{c}\mathrm{By} \\
\text { treatability }\end{array}$ & $\begin{array}{c}\mathrm{By} \\
\text { treatability }\end{array}$ & $\begin{array}{c}\text { W wall } n \\
\text { side }\end{array}$ & $\begin{array}{c}\text { W wall } n \\
\text { side }\end{array}$ & $\begin{array}{c}\text { Floor } 3^{\prime} \text { in } \\
\text { front }\end{array}$ \\
\hline \multicolumn{8}{|c|}{ Smear Gross Count (dpm/100 cm $\left.{ }^{2}\right)$} \\
\hline Beta/Gamma & $\cdots$ & -- & -- & $\cdots$ & $-\cdots$ & $\ldots$ & $-\cdots$ \\
\hline Alpha & 28,000 & 14,000 & 14,000 & 7,700 & 8,400 & 140,000 & 77,000 \\
\hline \multicolumn{8}{|c|}{ Gamma Exposure Rate (mR/hr) } \\
\hline $2.5 \mathrm{~cm}$ & 1.8 & 1.9 & 3.1 & 1.6 & $<0.5$ & 1.2 & 1.5 \\
\hline $30 \mathrm{~cm}$ & $<0.5$ & $<0.5$ & $<0.5$ & $<0.5$ & $<0.5$ & $<0.5$ & $<0.5$ \\
\hline \multicolumn{8}{|c|}{ Absorbed Dose Rate (mrad/hr) } \\
\hline $2.5 \mathrm{~cm}$ & 146 & 86 & 174 & 87 & 9 & 28 & 87 \\
\hline $30 \mathrm{~cm}$ & $<0.5$ & $<0.5$ & $<0.5$ & $<0.5$ & $<0.5$ & $<0.5$ & $<0.5$ \\
\hline \multicolumn{8}{|c|}{ GEA Measured Isotopic Activity $\left(\mu \mathrm{Ci} / 100 \mathrm{~cm}^{2}\right)$} \\
\hline${ }^{54} \mathrm{Mn}$ & $<6 . \mathrm{E}-05$ & $<8 . E-05$ & $<9 . \mathrm{E}-05$ & $<9 . \mathrm{E}-05$ & $<4 . E-05$ & $<2 . E-04$ & $<9 . \mathrm{E}-05$ \\
\hline${ }^{60} \mathrm{Co}$ & $<1 . E-04$ & $<2 . E-04$ & $2.93 E-04$ & $<2 . E-04$ & $<5 . E-05$ & $<9 . E-05$ & $4.06 \mathrm{E}-04$ \\
\hline${ }^{90} \mathrm{Sr}^{(\mathrm{a}, \mathrm{b})}$ & $6.60 \mathrm{E}-01$ & $3.71 E-01$ & $3.16 \mathrm{E}-01$ & $3.31 E-01$ & $5.83 \mathrm{E}-03$ & $1.27 E-01$ & $3.34 \mathrm{E}-01$ \\
\hline${ }^{108 \mathrm{~m}} \mathrm{Ag}$ & $-\cdots$ & $-\cdots$ & --- & --- & -- & -- & -- \\
\hline${ }^{125} \mathrm{Sb}$ & $<3 . \mathrm{E}-03$ & $<2 . E-03$ & $<3 . \mathrm{E}-03$ & $<3 . \mathrm{E}-03$ & $<4 . E-04$ & $<2 . \mathrm{E}-03$ & $<2 . E-03$ \\
\hline${ }^{134} \mathrm{Cs}$ & $4.09 E-03$ & $7.14 \mathrm{E}-04$ & $<2 E-04$ & $8.05 E-04$ & $2.69 \mathrm{E}-04$ & $5.55 E-03$ & $2.34 \mathrm{E}-03$ \\
\hline${ }^{137} \mathrm{Cs}$ & $1.10 \mathrm{E}+00$ & $2.38 \mathrm{E}-01$ & $5.26 \mathrm{E}-01$ & 5.77E-01 & $2.03 E-02$ & $2.11 \mathrm{E}-01$ & $7.04 \mathrm{E}-01$ \\
\hline${ }^{154} \mathrm{Eu}$ & $2.25 \mathrm{E}-03$ & 1.33E-03 & $2.25 \mathrm{E}-03$ & $1.60 \mathrm{E}-03$ & $9.19 \mathrm{E}-04$ & $6.98 \mathrm{E}-03$ & $3.96 \mathrm{E}-03$ \\
\hline${ }^{155} \mathrm{Eu}$ & $<1 . E-03$ & $<8 . E-04$ & $1.61 \mathrm{E}-03$ & $<2 . E-03$ & $2.60 \mathrm{E}-04$ & $2.10 \mathrm{E}-03$ & $<1 . \mathrm{E}-03$ \\
\hline${ }^{241} \mathrm{Am}$ & $1.51 \mathrm{E}-03$ & 1.37E-03 & $2.94 \mathrm{E}-03$ & $<2 . E-03$ & $3.53 \mathrm{E}-04$ & $3.67 E-03$ & $1.90 \mathrm{E}-03$ \\
\hline \multicolumn{8}{|c|}{ AEA Measured Isotopic Activity $\left(\mu \mathrm{Ci} / 100 \mathrm{~cm}^{2}\right)$} \\
\hline${ }^{239} \mathrm{Pu} /{ }^{240} \mathrm{Pu}$ & $5.69 \mathrm{E}-04$ & $7.14 \mathrm{E}-04$ & $-\cdots$ & $<1 . E-03$ & $<1 . E-04$ & $\cdots$ & $<1 . \mathrm{E}-03$ \\
\hline$\left.{ }^{238} \mathrm{Pu}\right|^{241} \mathrm{Am}$ & $3.27 E-03$ & $1.79 \mathrm{E}-03$ & -- & $4.18 \mathrm{E}-03$ & $2.03 \mathrm{E}-04$ & $\cdots$ & $9.20 \mathrm{E}-03$ \\
\hline${ }^{243} \mathrm{Cm} /{ }^{244} \mathrm{Cm}$ & $1.15 \mathrm{E}-02$ & $5.05 \mathrm{E}-03$ & $\ldots$ & $1.15 \mathrm{E}-02$ & $1.53 \mathrm{E}-03$ & -- & $2.90 \mathrm{E}-02$ \\
\hline${ }^{242} \mathrm{Cm}$ & $<3 . \mathrm{E}-05$ & $<1 . E-05$ & --- & $<1 . E-04$ & $<1 . E-05$ & -- & $<1$.E-04 \\
\hline \multicolumn{8}{|c|}{ Measured Gross Activitv $\left(\mu \mathrm{Ci} / 100 \mathrm{~cm}^{\frac{1}{2}}\right)$} \\
\hline Alpha & 1.53E-02 & $7.55 \mathrm{E}-03$ & $5.62 \mathrm{E}-03$ & $1.57 E-02$ & 1.73E-02 & $6.53 \mathrm{E}-02$ & $3.82 \mathrm{E}-02$ \\
\hline Beta/Gamma & $1.77 E+00$ & $6.11 \mathrm{E}-01$ & $8.46 E-01$ & $9.10 \mathrm{E}-01$ & $2.76 \mathrm{E}-02$ & $3.52 E-01$ & $1.04 \mathrm{E}+00$ \\
\hline Total & $1.78 \mathrm{E}+00$ & $6.19 \mathrm{E}-01$ & $8.51 \mathrm{E}-01$ & $9.26 \mathrm{E}-01$ & $4.49 \mathrm{E}-02$ & $4.18 \mathrm{E}-01$ & $1.08 \mathrm{E}+00$ \\
\hline \multicolumn{8}{|c|}{ Calculated Isotopic Activity $\left(\mu \mathrm{Ci} / 100 \mathrm{~cm}^{2}\right)$} \\
\hline${ }^{238} \mathrm{Pu}^{(\mathrm{c})}$ & $6.05 \mathrm{E}-04$ & $3.31 \mathrm{E}-04$ & -- & $7.73 \mathrm{E}-04$ & $3.76 \mathrm{E}-05$ & $\cdots$ & 1.70E-03 \\
\hline${ }^{239} \mathrm{Pu}^{(\mathrm{d})}$ & $2.87 E-04$ & $3.61 E-04$ & -- & $\cdots$ & $\cdots$ & $\cdots$ & -- \\
\hline${ }^{240} \mathrm{Pu}^{(\mathrm{e})}$ & $2.82 E-04$ & 3.53E-04 & -- & -- & -- & -- & $\cdots$ \\
\hline${ }^{241} \mathrm{Pu}^{(\mathrm{f})}$ & $1.41 \mathrm{E}-02$ & $1.76 \mathrm{E}-02$ & $\cdots$ & $\cdots$ & $\cdots$ & $\cdots$ & -- \\
\hline${ }^{242} \mathrm{Pu}^{(\mathrm{g})}$ & 4.71E-07 & 5.91E-07 & --- & --- & --- & --- & $\ldots$ \\
\hline${ }^{241} \mathrm{Am}^{(\mathrm{a}, \mathrm{h})}$ & $1.51 \mathrm{E}-03$ & $1.37 \mathrm{E}-03$ & $2.94 \mathrm{E}-03$ & $3.41 \mathrm{E}-03$ & $3.53 E-04$ & $3.67 \mathrm{E}-03$ & $1.90 \mathrm{E}-03$ \\
\hline${ }^{243} \mathrm{Cm}^{(\mathrm{i})}$ & $1.64 E-04$ & $7.22 E-05$ & --- & 1.64E-04 & $2.19 \mathrm{E}-05$ & $\cdots$ & $4.15 \mathrm{E}-04$ \\
\hline${ }^{244} \mathrm{Cm}^{(k)}$ & $1.13 \mathrm{E}-02$ & $4.98 E-03$ & $-\cdots$ & $1.13 E-02$ & $1.51 E-03$ & $\cdots$ & $2.86 \mathrm{E}-02$ \\
\hline${ }^{79} \mathrm{Se}^{(\mathrm{m})}$ & $3.56 \mathrm{E}-07$ & $3.23 \mathrm{E}-07$ & $6.94 \mathrm{E}-07$ & $8.04 \mathrm{E}-07$ & $8.33 E-08$ & $8.66 \mathrm{E}-07$ & $4.48 E-07$ \\
\hline${ }^{99} \mathrm{Tc}^{(\mathrm{n})}$ & $1.18 \mathrm{E}-05$ & $1.07 E-05$ & $2.30 E-05$ & 2.67E-05 & $2.76 \mathrm{E}-06$ & $2.87 \mathrm{E}-05$ & $1.49 \mathrm{E}-05$ \\
\hline
\end{tabular}

Assumptions: (a) measured activity used, if available

(b) ${ }^{90} \mathrm{Sr}={ }^{137} \mathrm{Cs} \times 6.00 \mathrm{E}-01$

(c) ${ }^{238} \mathrm{Pu}={ }^{238} \mathrm{Pu} /{ }^{241} \mathrm{Am} \times 1.85 \mathrm{E}-01$
(d) ${ }^{239} \mathrm{Pu}={ }^{239 / 240} \mathrm{Pu} \times 5.05 \mathrm{E}-01$
(e) ${ }^{240} \mathrm{Pu}={ }^{239 / 240} \mathrm{Pu} \times 4.95 \mathrm{E}-01$
(f) ${ }^{241} \mathrm{Pu}={ }^{239 / 240} \mathrm{Pu} \times 2.47 \mathrm{E}+01$
(g) ${ }^{242} \mathrm{Pu}={ }^{239 / 240} \mathrm{Pu} \times 8.28 \mathrm{E}-04$

(h) ${ }^{241} \mathrm{Am}={ }^{238} \mathrm{Pu} /{ }^{241} \mathrm{Am} \times 8.15 \mathrm{E}-01$

(j) ${ }^{243} \mathrm{Cm}={ }^{243 / 244} \mathrm{Cm} \times 1.43 \mathrm{E}-02$

(k) ${ }^{244} \mathrm{Cm}={ }^{243 / 244} \mathrm{Cm} \times 9.86 \mathrm{E}-01$

(m) ${ }^{79} \mathrm{Se}={ }^{241} \mathrm{Am} \times 2.36 \mathrm{E}-04$

(n) ${ }^{99} \mathrm{Tc}={ }^{241} \mathrm{Am} \times 7.83 \mathrm{E}-03$

(p) total $=$ removable $\times 3.00 \mathrm{E}+01$ 


\section{REC C-Cell}

\begin{tabular}{|c|c|c|c|c|c|c|c|}
\hline \multirow{3}{*}{$\begin{array}{c}\text { Sample No. } \\
\text { Sample } \\
\text { Location }\end{array}$} & \multirow{3}{*}{$\begin{array}{c}21 b \\
\begin{array}{c}\text { Floor } 3^{\prime} \text { in } \\
\text { front }\end{array}\end{array}$} & \multirow{3}{*}{\begin{tabular}{|c|}
$22 a$ \\
$2^{\prime} n$ of filter
\end{tabular}} & \multirow{3}{*}{$\frac{22 b}{22^{\prime} n \text { of filter }}$} & \multicolumn{4}{|c|}{ Estimated Isotopic Activity $\left(\mu \mathrm{Ci} / 100 \mathrm{~cm}^{2}\right)^{(\mathrm{p})}$} \\
\hline & & & & \multicolumn{2}{|c|}{ Removable } & \multicolumn{2}{|c|}{ Total } \\
\hline & & & & Average & $\operatorname{Max}$ & Average & $\operatorname{Max}$ \\
\hline & \multicolumn{7}{|c|}{ Smear Gross Count (dpm/100 $\mathrm{cm}^{2}$ ) } \\
\hline Beta/Gamma & $\cdots$ & -- & --- & $\mathrm{NA}$ & $\mathrm{NA}$ & $\mathrm{NA}$ & $\mathrm{NA}$ \\
\hline Alpha & 140,000 & 98,000 & 56,000 & NA & NA & NA & NA \\
\hline \multicolumn{8}{|c|}{ Gamma Exposure Rate (mR/hr) } \\
\hline $2.5 \mathrm{~cm}$ & 2.5 & $<0.5$ & $<0.5$ & NA & NA & NA & $\mathrm{NA}$ \\
\hline $30 \mathrm{~cm}$ & $<0.5$ & $<0.5$ & $<0.5$ & $\mathrm{NA}$ & NA & NA & NA \\
\hline \multicolumn{8}{|c|}{ Absorbed Dose Rate (mrad/hr) } \\
\hline $2.5 \mathrm{~cm}$ & 88 & 4.5 & 15.0 & $\mathrm{NA}$ & $\mathrm{NA}$ & NA & $\mathrm{NA}$ \\
\hline $30 \mathrm{~cm}$ & $<0.5$ & $<0.5$ & $<0.5$ & $\mathrm{NA}$ & NA & $\mathrm{NA}$ & NA \\
\hline \multicolumn{8}{|c|}{ GEA Measured Isotopic Activity $\left(\mu \mathrm{Ci} / 100 \mathrm{~cm}^{2}\right)$} \\
\hline${ }^{54} \mathrm{Mn}$ & $<2 . \mathrm{E}-04$ & $<7 . E-05$ & $<6 . E-05$ & ND & ND & ND & ND \\
\hline${ }^{60} \mathrm{Co}$ & $8.49 E-04$ & $4.22 E-05$ & $<2 . E-05$ & $3.98 \mathrm{E}-04$ & $8.49 E-04$ & $1.19 \mathrm{E}-02$ & $2.55 E-02$ \\
\hline${ }^{90} \mathrm{Sr}^{(\mathrm{a}, \mathrm{b})}$ & $5.43 \mathrm{E}-01$ & $1.09 E-03$ & $4.75 E-02$ & $2.74 \mathrm{E}-01$ & $6.60 \mathrm{E}-01$ & $8.21 \mathrm{E}+00$ & $1.98 \mathrm{E}+01$ \\
\hline${ }^{108 \mathrm{~m}} \mathrm{Ag}$ & -- & $-\cdots$ & --- & --- & --- & -- & -- \\
\hline${ }^{125} \mathrm{Sb}$ & $<3 . \mathrm{E}-03$ & $<7 . E-04$ & $<7 . E-04$ & ND & ND & ND & ND \\
\hline${ }^{134} \mathrm{Cs}$ & $3.65 \mathrm{E}-03$ & $2.34 \mathrm{E}-03$ & $1.10 \mathrm{E}-03$ & $2.32 \mathrm{E}-03$ & $5.55 \mathrm{E}-03$ & $6.95 \mathrm{E}-02$ & $1.67 \mathrm{E}-01$ \\
\hline${ }^{137} \mathrm{Cs}$ & $9.05 E-01$ & $9.09 E-02$ & $7.91 E-02$ & $4.45 \mathrm{E}-01$ & $1.10 E+00$ & $1.34 E+01$ & $3.30 E+01$ \\
\hline${ }^{154} \mathrm{Eu}$ & $6.06 \mathrm{E}-03$ & $2.77 E-03$ & $1.98 \mathrm{E}-03$ & $3.01 E-03$ & $6.98 \mathrm{E}-03$ & $9.03 E-02$ & $2.09 \mathrm{E}-01$ \\
\hline${ }^{155} \mathrm{Eu}$ & $1.96 E-03$ & $8.66 \mathrm{E}-04$ & $<4 . E-04$ & $1.36 \mathrm{E}-03$ & $2.10 \mathrm{E}-03$ & $4.08 \mathrm{E}-02$ & $6.30 \mathrm{E}-02$ \\
\hline${ }^{241} \mathrm{Am}$ & $2.94 \mathrm{E}-03$ & $4.09 \mathrm{E}-04$ & $1.09 \mathrm{E}-03$ & $1.80 E-03$ & $3.67 \mathrm{E}-03$ & $5.39 \mathrm{E}-02$ & $1.10 \mathrm{E}-01$ \\
\hline \multicolumn{8}{|c|}{ AEA Measured Isotopic Activity $\left(\mu \mathrm{Ci} / 100 \mathrm{~cm}^{2}\right)$} \\
\hline${ }^{239} \mathrm{Pu} /{ }^{240} \mathrm{Pu}$ & -- & $<1 . E-05$ & \begin{tabular}{c|c}
-- \\
\end{tabular} & $6.42 E-04$ & $7.14 \mathrm{E}-04$ & $1.92 E-02$ & $2.14 E-02$ \\
\hline${ }^{238} \mathrm{Pu} /{ }^{241} \mathrm{Am}$ & $\ldots$ & $4.64 \mathrm{E}-05$ & -- & $3.11 E-03$ & $9.20 \mathrm{E}-03$ & $9.34 \mathrm{E}-02$ & $2.76 \mathrm{E}-01$ \\
\hline${ }^{243} \mathrm{Cm} /{ }^{244} \mathrm{Cm}$ & $\cdots$ & 3.97E-04 & $\ldots$ & $9.83 E-03$ & $2.90 \mathrm{E}-02$ & $2.95 \mathrm{E}-01$ & $8.70 \mathrm{E}-01$ \\
\hline${ }^{242} \mathrm{Cm}$ & -- & $\begin{array}{l}\text { - E-05 } \\
\end{array}$ & -- & ND & ND & ND & ND \\
\hline \multicolumn{8}{|c|}{ Measured Gross Activity $\left(\mu \mathrm{Ci} / 100 \mathrm{~cm}^{2}\right)$} \\
\hline Alpha & $7.90 \mathrm{E}-02$ & $4.43 E-04$ & $1.83 \mathrm{E}-02$ & $2.63 \mathrm{E}-02$ & $7.90 \mathrm{E}-02$ & $7.88 \mathrm{E}-01$ & $2.37 \mathrm{E}+00$ \\
\hline Beta/Gamma & $1.46 \mathrm{E}+00$ & $9.80 \mathrm{E}-02$ & $1.30 \mathrm{E}-01$ & $7.25 E-01$ & $1.77 E+00$ & $2.17 E+01$ & $5.30 E+01$ \\
\hline Total & $1.54 \mathrm{E}+00$ & $9.85 \mathrm{E}-02$ & $1.48 \mathrm{E}-01$ & $7.51 E-01$ & $1.78 \mathrm{E}+00$ & $2.25 \mathrm{E}+01$ & $5.34 \mathrm{E}+01$ \\
\hline \multicolumn{8}{|c|}{ Calculated Isotopic Activity $\left(\mu \mathrm{Ci} / 100 \mathrm{~cm}^{2}\right)$} \\
\hline${ }^{238} \mathrm{Pu}^{(\mathrm{c})}$ & -- & $8.58 E-06$ & $-\cdots$ & $5.76 E-04$ & $1.70 \mathrm{E}-03$ & $1.73 \mathrm{E}-02$ & $5.11 \mathrm{E}-02$ \\
\hline${ }^{239} \mathrm{Pu}^{(\mathrm{d})}$ & $\ldots$ & $\ldots$ & -- & $3.24 \mathrm{E}-04$ & $3.61 E-04$ & $9.72 E-03$ & $1.08 \mathrm{E}-02$ \\
\hline${ }^{240} \mathrm{Pu}^{(\mathrm{e})}$ & -- & $\cdots$ & $\cdots$ & $3.18 E-04$ & $3.53 E-04$ & $9.53 E-03$ & $1.06 \mathrm{E}-02$ \\
\hline${ }^{241} \mathrm{Pu}^{(\mathrm{f})}$ & $\cdots$ & -- & -- & $1.58 \mathrm{E}-02$ & $1.76 \mathrm{E}-02$ & $4.75 E-01$ & $5.29 E-01$ \\
\hline${ }^{242} \mathrm{Pu}^{(\mathrm{g})}$ & $\cdots$ & $\cdots$ & $\ldots$ & $5.31 E-07$ & $5.91 \mathrm{E}-07$ & $1.59 \mathrm{E}-05$ & $1.77 \mathrm{E}-05$ \\
\hline${ }^{241} \mathrm{Am}^{(\mathrm{a}, \mathrm{h})}$ & $2.94 E-03$ & $4.09 \mathrm{E}-04$ & $1.09 \mathrm{E}-03$ & $1.96 \mathrm{E}-03$ & $3.67 \mathrm{E}-03$ & $5.88 \mathrm{E}-02$ & $1.10 \mathrm{E}-01$ \\
\hline${ }^{243} \mathrm{Cm}^{(0)}$ & $\cdots$ & $5.68 \mathrm{E}-06$ & --- & $1.41 \mathrm{E}-04$ & $4.15 \mathrm{E}-04$ & $4.22 E-03$ & $1.24 \mathrm{E}-02$ \\
\hline${ }^{244} \mathrm{Cm}^{(\mathrm{k})}$ & $\cdots$ & $3.91 E-04$ & $\cdots$ & $9.69 \mathrm{E}-03$ & $2.86 \mathrm{E}-02$ & $2.91 E-01$ & $8.58 \mathrm{E}-01$ \\
\hline${ }^{79} \mathrm{Se}^{(\mathrm{m})}$ & $6.94 \mathrm{E}-07$ & $9.65 E-08$ & $2.57 E-07$ & $4.62 E-07$ & $8.66 \mathrm{E}-07$ & $1.39 \mathrm{E}-05$ & $2.60 \mathrm{E}-05$ \\
\hline${ }^{99} \mathrm{Tc}^{(\mathrm{n})}$ & $2.30 E-05$ & $3.20 \mathrm{E}-06$ & $8.53 E-06$ & $1.53 \mathrm{E}-05$ & $2.87 \mathrm{E}-05$ & $4.60 \mathrm{E}-04$ & $8.62 E-04$ \\
\hline
\end{tabular}

Assumptions: (a) measured activity used, if available

(b) ${ }^{90} \mathrm{Sr}={ }^{137} \mathrm{Cs} \times 6.00 \mathrm{E}-01$

(c) ${ }^{238} \mathrm{Pu}={ }^{238} \mathrm{Pu} /{ }^{241} \mathrm{Am} \times 1.85 \mathrm{E}-01$

(d) ${ }^{239} \mathrm{Pu}={ }^{239 / 240} \mathrm{Pu} \times 5.05 \mathrm{E}-01$

(e) ${ }^{240} \mathrm{Pu}={ }^{239 / 240} \mathrm{Pu} \times 4.95 \mathrm{E}-01$

(f) ${ }^{241} \mathrm{Pu}={ }^{239 / 240} \mathrm{Pu} \times 2.47 \mathrm{E}+01$

(g) ${ }^{242} \mathrm{Pu}={ }^{239 / 240} \mathrm{Pu} \times 8.28 \mathrm{E}-04$ (h) ${ }^{241} \mathrm{Am}={ }^{238} \mathrm{Pu} /{ }^{241} \mathrm{Am} \times 8.15 \mathrm{E}-01$

(j) ${ }^{243} \mathrm{Cm}={ }^{243 / 244} \mathrm{Cm} \times 1.43 \mathrm{E}-02$

(k) ${ }^{244} \mathrm{Cm}={ }^{243 / 244} \mathrm{Cm} \times 9.86 \mathrm{E}-01$

(m) ${ }^{79} \mathrm{Se}={ }^{241} \mathrm{Am} \times 2.36 \mathrm{E}-04$

(n) ${ }^{99} \mathrm{Tc}={ }^{241} \mathrm{Am} \times 7.83 \mathrm{E}-03$

(p) total $=$ removable $\times 3.00 E+01$ 


\section{REC D-Cell}

\begin{tabular}{|c|c|c|c|c|c|c|c|}
\hline Sample No. & $26 a$ & $26 b$ & $27 a$ & $27 b$ & $28 \mathbf{a}$ & $28 b$ & $30 a$ \\
\hline $\begin{array}{l}\text { Sample } \\
\text { Location }\end{array}$ & $\begin{array}{c}\text { S wall } \\
\text { below } \\
\text { window }\end{array}$ & $\begin{array}{c}\text { S wall } \\
\text { below } \\
\text { window }\end{array}$ & $\begin{array}{c}\text { E wall; } \\
\text { window } \\
\text { height }\end{array}$ & $\begin{array}{l}\text { E wall; } \\
\text { window } \\
\text { height }\end{array}$ & $\begin{array}{l}\text { FIr E side; } \\
2 \text { ' in front } \\
\text { of window }\end{array}$ & $\begin{array}{l}\text { Flr E side; } \\
2 \text { in front } \\
\text { of window }\end{array}$ & $\begin{array}{l}\text { W wall; } 3 \text { ' } \\
\text { above flr; } S \\
\text { of filter }\end{array}$ \\
\hline \multicolumn{8}{|c|}{ Smear Gross Count $\left(\mathrm{dpm} / 100 \mathrm{~cm}^{2}\right)$} \\
\hline Beta/Gamma & --- & -- & --- & $\ldots$ & $-\ldots$ & $\ldots$ & 70,000 \\
\hline Alpha & 315,000 & 490,000 & 350,000 & 70,000 & $>700,000$ & $>700,000$ & 70,000 \\
\hline \multicolumn{8}{|c|}{ Gamma Exposure Rate (mR/hr) } \\
\hline $2.5 \mathrm{~cm}$ & 45 & 60 & 45 & 23 & 540 & 105 & 105 \\
\hline $30 \mathrm{~cm}$ & 9 & 8 & 8 & 10 & 50 & 10 & 10 \\
\hline \multicolumn{8}{|c|}{ Absorbed Dose Rate (mrad/hr) } \\
\hline $2.5 \mathrm{~cm}$ & 2,355 & 2,340 & 2,955 & 1,478 & 74,460 & 6,795 & 5,295 \\
\hline $30 \mathrm{~cm}$ & 72 & 59 & 59 & 25 & 1,700 & 220 & 100 \\
\hline \multicolumn{8}{|c|}{ GEA Measured Isotopic Activity $\left(\mu \mathrm{Ci} / 100 \mathrm{~cm}^{2}\right)$} \\
\hline${ }^{54} \mathrm{Mn}$ & $<3 . E-03$ & $<3 . \mathrm{E}-03$ & $<4 . E-03$ & $<2 . E-03$ & $<2 . \mathrm{E}-01$ & $<9 . \mathrm{E}-03$ & $<7 . \mathrm{E}-03$ \\
\hline${ }^{60} \mathrm{Co}$ & $2.47 \mathrm{E}-02$ & $4.73 \mathrm{E}-02$ & $8.35 E-02$ & $2.81 E-02$ & $1.31 \mathrm{E}+00$ & $9.41 E-02$ & $1.45 E-02$ \\
\hline${ }^{90} \mathrm{Sr}^{(\mathrm{a}, \mathrm{b})}$ & $9.20 E+00$ & $6.04 \mathrm{E}-02$ & $1.33 E+01$ & $2.36 \mathrm{E}-02$ & $2.50 E+02$ & $1.21 \mathrm{E}-01$ & $1.39 E-01$ \\
\hline${ }^{108 m} \mathrm{Ag}$ & --- & $1.59 \mathrm{E}-02$ & $-\cdots$ & $-\cdots$ & $\ldots$ & $\ldots$ & $\ldots$ \\
\hline${ }^{125} \mathrm{Sb}$ & $<5 . E-02$ & $<5 . E-02$ & $<6 . E-02$ & $<4 . E-02$ & $<4 . E+00$ & $<2 . E-01$ & $<2 . E-01$ \\
\hline${ }^{134} \mathrm{Cs}$ & 1.69E-02 & $2.92 \mathrm{E}-02$ & $2.94 \mathrm{E}-02$ & $9.75 E-03$ & $<3 . E-01$ & $4.44 E-02$ & $2.28 E-02$ \\
\hline${ }^{137} \mathrm{Cs}$ & $1.31 \mathrm{E}+01$ & $1.70 \mathrm{E}+01$ & $2.81 E+01$ & $1.26 \mathrm{E}+01$ & $1.25 \mathrm{E}+03$ & $4.92 E+01$ & $4.36 \mathrm{E}+01$ \\
\hline${ }^{154} \mathrm{Eu}$ & $4.20 \mathrm{E}-02$ & $8.43 E-02$ & $6.34 \mathrm{E}-02$ & $1.84 E-02$ & $6.99 E+00$ & $8.24 E-02$ & $1.46 \mathrm{E}-02$ \\
\hline${ }^{155} \mathrm{Eu}$ & $<3 . E-02$ & $<3 . E-02$ & $<4 . \mathrm{E}-02$ & $<3 . E-02$ & $<2 . E+00$ & $<2 . \mathrm{E}-01$ & $<2 . E-01$ \\
\hline${ }^{241} \mathrm{Am}$ & $7.29 E-02$ & $1.51 \mathrm{E}-01$ & 1.13E-01 & $<4 . E-02$ & $<2 . E+00$ & $<2 . E-01$ & $<2 . E-01$ \\
\hline \multicolumn{8}{|c|}{ AEA Measured Isotopic Activity $\left(\mu \mathrm{Ci} / 100 \mathrm{~cm}^{2}\right)$} \\
\hline${ }^{239} \mathrm{Pu} /{ }^{240} \mathrm{Pu}$ & $2.00 \mathrm{E}-02$ & 4.02E-02 & 3.50E-02 & $9.60 \mathrm{E}-03$ & $1.39 \mathrm{E}-01$ & $2.44 \mathrm{E}-02$ & $5.32 \mathrm{E}-03$ \\
\hline${ }^{238} \mathrm{Pu} / /^{241} \mathrm{Am}$ & 1.27E-01 & $2.09 E-01$ & $1.96 \mathrm{E}-01$ & $4.69 \mathrm{E}-02$ & $9.29 \mathrm{E}-01$ & $1.37 \mathrm{E}-01$ & $3.39 \mathrm{E}-02$ \\
\hline${ }^{243} \mathrm{Cm} /{ }^{244} \mathrm{Cm}$ & $1.20 \mathrm{E}-01$ & $2.66 \mathrm{E}-01$ & $1.54 \mathrm{E}-01$ & $5.24 \mathrm{E}-02$ & $1.54 \mathrm{E}+00$ & $2.39 E-01$ & $4.27 \mathrm{E}-02$ \\
\hline${ }^{242} \mathrm{Cm}$ & $5.95 \mathrm{E}-04$ & $8.49 \mathrm{E}-04$ & $5.26 \mathrm{E}-04$ & $1.36 \mathrm{E}-04$ & $5.75 \mathrm{E}-03$ & 4.67E-04 & $2.16 \mathrm{E}-04$ \\
\hline \multicolumn{8}{|c|}{ Measured Gross Activity $\left(\mu \mathrm{Ci} / 100 \mathrm{~cm}^{2}\right)$} \\
\hline Alpha & 2.67E-01 & 5.19E-01 & $3.20 \mathrm{E}-01$ & 1.09E-01 & $2.28 \mathrm{E}+00$ & $4.04 \mathrm{E}-01$ & $8.28 \mathrm{E}-02$ \\
\hline Beta/Gamma & $2.24 \mathrm{E}+01$ & $1.72 E+01$ & $4.16 \mathrm{E}+01$ & $1.27 \mathrm{E}+01$ & $1.51 E+03$ & $4.95 \mathrm{E}+01$ & $4.38 \mathrm{E}+01$ \\
\hline Total & $2.27 \mathrm{E}+01$ & $1.78 \mathrm{E}+01$ & $4.19 \mathrm{E}+01$ & $1.28 \mathrm{E}+01$ & $1.51 E+03$ & $4.99 \mathrm{E}+01$ & $4.39 \mathrm{E}+01$ \\
\hline \multicolumn{8}{|c|}{ Calculated Isotopic Activitv $\left(\mu \mathrm{Ci} / 100 \mathrm{~cm}^{2}\right)$} \\
\hline${ }^{238} \mathrm{Pu}^{(\mathrm{c})}$ & $2.35 \mathrm{E}-02$ & $3.87 E-02$ & $3.63 E-02$ & $8.68 \mathrm{E}-03$ & $1.72 \mathrm{E}-01$ & $2.53 E-02$ & $6.27 \mathrm{E}-03$ \\
\hline${ }^{239} \mathrm{Pu}^{(\mathrm{d})}$ & $1.01 E-02$ & $2.03 E-02$ & $1.77 \mathrm{E}-02$ & $4.85 \mathrm{E}-03$ & $7.02 \mathrm{E}-02$ & $1.23 \mathrm{E}-02$ & $2.69 \mathrm{E}-03$ \\
\hline${ }^{240} \mathrm{Pu}^{(\mathrm{e})}$ & $9.90 \mathrm{E}-03$ & $1.99 \mathrm{E}-02$ & $1.73 \mathrm{E}-02$ & 4.75E-03 & $6.88 \mathrm{E}-02$ & $1.21 \mathrm{E}-02$ & $2.63 \mathrm{E}-03$ \\
\hline${ }^{241} \mathrm{Pu}^{(f)}$ & $4.94 \mathrm{E}-01$ & 9.93E-01 & $8.65 \mathrm{E}-01$ & $2.37 \mathrm{E}-01$ & $3.43 \mathrm{E}+00$ & $6.03 E-01$ & $1.31 \mathrm{E}-01$ \\
\hline${ }^{242} \mathrm{Pu}^{(\mathrm{g})}$ & 1.66E-05 & 3.33E-05 & $2.90 \mathrm{E}-05$ & $7.95 E-06$ & $1.15 \mathrm{E}-04$ & $2.02 E-05$ & $4.40 E-06$ \\
\hline${ }^{241} \mathrm{Am}^{(a, h)}$ & $7.29 \mathrm{E}-02$ & 1.51E-01 & $1.13 \mathrm{E}-01$ & $3.82 E-02$ & $7.57 \mathrm{E}-01$ & $1.12 \mathrm{E}-01$ & $2.76 \mathrm{E}-02$ \\
\hline${ }^{243} \mathrm{Cm}^{(0)}$ & $1.72 E-03$ & $3.80 \mathrm{E}-03$ & $2.20 \mathrm{E}-03$ & $7.49 \mathrm{E}-04$ & $2.20 \mathrm{E}-02$ & $3.42 E-03$ & $6.11 \mathrm{E}-04$ \\
\hline${ }^{244} \mathrm{Cm}^{(\mathrm{k})}$ & $1.18 \mathrm{E}-01$ & $2.62 \mathrm{E}-01$ & $1.52 \mathrm{E}-01$ & $5.17 E-02$ & $1.52 E+00$ & $2.36 \mathrm{E}-01$ & $4.21 \mathrm{E}-02$ \\
\hline${ }^{79} \mathrm{Se}^{(\mathrm{m})}$ & $1.72 E-05$ & $3.56 \mathrm{E}-05$ & $2.67 E-05$ & $9.02 E-06$ & 1.79E-04 & $2.64 \mathrm{E}-05$ & $6.52 E-06$ \\
\hline${ }^{99} \mathrm{Tc}^{(\mathrm{n})}$ & $5.71 E-04$ & $1.18 \mathrm{E}-03$ & $8.85 E-04$ & 2.99E-04 & $5.93 E-03$ & $8.74 \mathrm{E}-04$ & $2.16 \mathrm{E}-04$ \\
\hline
\end{tabular}

Assumptions: (a) measured activity used, if available

(b) ${ }^{90} \mathrm{Sr}={ }^{137} \mathrm{Cs} \times 6.00 \mathrm{E}-01$

(c) ${ }^{238} \mathrm{Pu}={ }^{238} \mathrm{Pu} /{ }^{241} \mathrm{Am} \times 1.85 \mathrm{E}-01$

(d) ${ }^{239} \mathrm{Pu}={ }^{239 / 240} \mathrm{Pu} \times 5.05 \mathrm{E}-01$

(e) ${ }^{240} \mathrm{Pu}={ }^{239 / 240} \mathrm{Pu} \times 4.95 \mathrm{E}-01$

(f) ${ }^{241} \mathrm{Pu}={ }^{239 / 240} \mathrm{Pu} \times 2.47 \mathrm{E}+01$

(g) ${ }^{242} \mathrm{Pu}={ }^{239 / 240} \mathrm{Pu} \times 8.28 \mathrm{E}-04$ (h) ${ }^{241} \mathrm{Am}={ }^{238} \mathrm{Pu} /{ }^{241} \mathrm{Am} \times 8.15 \mathrm{E}-01$

(j) ${ }^{243} \mathrm{Cm}={ }^{243 / 244} \mathrm{Cm} \times 1.43 \mathrm{E}-02$

(k) ${ }^{244} \mathrm{Cm}={ }^{243 / 244} \mathrm{Cm} \times 9.86 \mathrm{E}-01$

$(\mathrm{m}){ }^{79} \mathrm{Se}={ }^{241} \mathrm{Am} \times 2.36 \mathrm{E}-04$

(n) ${ }^{99} \mathrm{Tc}={ }^{241} \mathrm{Am} \times 7.83 \mathrm{E}-03$

$(p)$ total $=$ removable $\times 3.00 E+01$ 
REC D-Cell

\begin{tabular}{|c|c|c|c|c|c|c|c|}
\hline Sample No. & & $31 a$ & 31b & \multicolumn{4}{|c|}{ Estimated Isotopic Activity $\left(\mu \mathrm{Ci} / 100 \mathrm{~cm}^{2}\right)^{(p)}$} \\
\hline \multirow{2}{*}{$\begin{array}{c}\text { Sample } \\
\text { Location }\end{array}$} & \multirow{2}{*}{$\begin{array}{c}\text { W wall; } 3^{1} \\
\text { above flr; S } \\
\text { of filter }\end{array}$} & \multirow{2}{*}{$\begin{array}{c}\text { Lead } \\
\text { Storage }\end{array}$} & \multirow{2}{*}{$\begin{array}{c}\text { Lead } \\
\text { Storage }\end{array}$} & \multicolumn{2}{|c|}{ Removable } & \multicolumn{2}{|c|}{ Total } \\
\hline & & & & Average & Max & Average & $\operatorname{Max}$ \\
\hline \multicolumn{8}{|c|}{ Smear Gross Count (dpm/100 $\left.\mathrm{cm}^{2}\right)$} \\
\hline Beta/Gamma & \begin{tabular}{|c|}
-- \\
\end{tabular} &.-- & --- & NA & NA & NA & NA \\
\hline Alpha & 70,000 & $>700,000$ & 280,000 & $\mathrm{NA}$ & $\mathrm{NA}$ & $\mathrm{NA}$ & $\overline{N A}$ \\
\hline \multicolumn{8}{|c|}{ Gamma Exposure Rate (mR/hr) } \\
\hline $2.5 \mathrm{~cm}$ & 68 & 900 & 330 & NA & NA & NA & NA \\
\hline $30 \mathrm{~cm}$ & 6 & 25 & 12 & $\mathrm{NA}$ & NA & NA & NA \\
\hline \multicolumn{8}{|c|}{ Absorbed Dose Rate (mrad/hr) } \\
\hline $2.5 \mathrm{~cm}$ & 5,033 & 59,100 & 29,670 & NA & NA & NA & NA \\
\hline $30 \mathrm{~cm}$ & 108 & 1,000 & 426 & NA & $\mathrm{NA}$ & $\mathrm{NA}$ & $\mathrm{NA}$ \\
\hline \multicolumn{8}{|c|}{ GEA Measured Isotopic Activity $\left(\mu \mathrm{Ci} / 100 \mathrm{~cm}^{2}\right)$} \\
\hline${ }^{54} \mathrm{Mn}$ & $<2 . E-03$ & $<6 . \mathrm{E}-02$ & $<3 . \mathrm{E}-02$ & ND & ND & ND & ND \\
\hline${ }^{60} \mathrm{Co}$ & $7.28 \mathrm{E}-03$ & 1.37E-01 & $6.44 \mathrm{E}-02$ & $1.81 \mathrm{E}-01$ & $1.31 \mathrm{E}+00$ & $5.43 \mathrm{E}+00$ & $3.93 E+01$ \\
\hline${ }^{90} \mathrm{Sr}^{(\mathrm{a}, \mathrm{b})}$ & $6.62 E+00$ & $1.76 \mathrm{E}+02$ & $6.86 \mathrm{E}+01$ & $5.24 E+01$ & $2.50 E+02$ & $1.57 E+03$ & $7.50 E+03$ \\
\hline${ }^{108 \mathrm{~m}} \mathrm{Ag}$ & -- & $\ldots$ & --- & $1.59 \mathrm{E}-02$ & $1.59 \mathrm{E}-02$ & $4.77 E-01$ & 4.77E-01 \\
\hline${ }^{125} \mathrm{Sb}$ & $<4 . \mathrm{E}-02$ & $<8 . E-01$ & $<6 . E-01$ & ND & ND & ND & ND \\
\hline${ }^{134} \mathrm{Cs}$ & $1.78 \mathrm{E}-03$ & 2.17E-01 & $1.09 \mathrm{E}-01$ & $5.34 E-02$ & 2.17E-01 & $1.60 \mathrm{E}+00$ & $6.51 E+00$ \\
\hline${ }^{137} \mathrm{Cs}$ & $1.21 \mathrm{E}+01$ & $4.57 E+02$ & $2.39 E+02$ & $2.12 E+02$ & $1.25 E+03$ & $6.37 E+03$ & $3.75 E+04$ \\
\hline${ }^{154} \mathrm{Eu}$ & 1.19E-02 & 1.80E-01 & $6.26 \mathrm{E}-02$ & $7.55 \mathrm{E}-01$ & $6.99 E+00$ & $2.26 \mathrm{E}+01$ & $2.10 E+02$ \\
\hline${ }^{155} \mathrm{Eu}$ & $<3 . E-02$ & $<6 . \mathrm{E}-01$ & $<4 . E-01$ & ND & ND & ND & ND \\
\hline${ }^{241} \mathrm{Am}$ & $4.01 \mathrm{E}-02$ & $\cdots$ & --- & $9.43 \mathrm{E}-02$ & $1.51 \mathrm{E}-01$ & $2.83 E+00$ & $4.53 E+00$ \\
\hline \multicolumn{8}{|c|}{ AEA Measured Isotopic Activity $\left(\mu \mathrm{Ci} / 100 \mathrm{~cm}^{2}\right)$} \\
\hline${ }^{239} \mathrm{Pu} /{ }^{240} \mathrm{Pu}$ & $3.57 \mathrm{E}-02$ & $2.18 \mathrm{E}-02$ & $1.36 \mathrm{E}-02$ & $3.45 E-02$ & $1.39 \mathrm{E}-01$ & $1.03 E+00$ & $4.17 E+00$ \\
\hline${ }^{238} \mathrm{Pu} /{ }^{241} \mathrm{Am}$ & $4.60 \mathrm{E}-02$ & 2.53E-01 & $6.84 \mathrm{E}-02$ & 2.05E-01 & $9.29 \mathrm{E}-01$ & $6.14 \mathrm{E}+00$ & $2.79 E+01$ \\
\hline${ }^{243} \mathrm{Cm} /{ }^{244} \mathrm{Cm}$ & $1.11 \mathrm{E}-02$ & $1.92 \mathrm{E}-01$ & $6.84 \mathrm{E}-02$ & $2.69 \mathrm{E}-01$ & $1.54 \mathrm{E}+00$ & $8.06 E+00$ & $4.62 E+01$ \\
\hline${ }^{242} \mathrm{Cm}$ & $5.85 \mathrm{E}-05$ & $1.75 \mathrm{E}-03$ & $4.24 \mathrm{E}-04$ & $1.08 \mathrm{E}-03$ & $5.75 \mathrm{E}-03$ & $3.23 \mathrm{E}-02$ & $1.73 \mathrm{E}-01$ \\
\hline \multicolumn{8}{|c|}{ Measured Gross Activity $\left(\mu \mathrm{Ci} / 100 \mathrm{~cm}^{2}\right)$} \\
\hline Alpha & $7.81 \mathrm{E}-02$ & $4.71 E-01$ & 1.79E-01 & $4.71 \mathrm{E}-01$ & $2.28 \mathrm{E}+00$ & $1.41 \mathrm{E}+01$ & $6.84 \mathrm{E}+01$ \\
\hline Beta/Gamma & 1.87E+01 & $6.34 \mathrm{E}+02$ & $3.08 \mathrm{E}+02$ & $2.66 \mathrm{E}+02$ & $1.51 E+03$ & $7.97 E+03$ & $4.52 E+04$ \\
\hline Total & $1.88 \mathrm{E}+01$ & $6.34 \mathrm{E}+02$ & $3.08 \mathrm{E}+02$ & $2.66 \mathrm{E}+02$ & $1.51 E+03$ & $7.98 \mathrm{E}+03$ & $4.53 E+04$ \\
\hline \multicolumn{8}{|c|}{ Calculated Isotopic Activity $\left(\mu \mathrm{Ci} / 100 \mathrm{~cm}^{2}\right)$} \\
\hline${ }^{238} \mathrm{Pu}^{(\mathrm{c})}$ & $8.51 \mathrm{E}-03$ & $4.68 \mathrm{E}-02$ & $1.27 \mathrm{E}-02$ & $3.79 E-02$ & $1.72 \mathrm{E}-01$ & $1.14 \mathrm{E}+00$ & $5.16 \mathrm{E}+00$ \\
\hline${ }^{239} \mathrm{Pu}^{(\mathrm{d})}$ & $1.80 \mathrm{E}-02$ & $1.10 \mathrm{E}-02$ & $6.87 \mathrm{E}-03$ & $1.74 \mathrm{E}-02$ & $7.02 \mathrm{E}-02$ & $5.22 \mathrm{E}-01$ & $2.11 E+00$ \\
\hline${ }^{240} \mathrm{Pu}^{(\mathrm{e})}$ & 1.77E-02 & $1.08 \mathrm{E}-02$ & $6.73 \mathrm{E}-03$ & $1.71 E-02$ & $6.88 \mathrm{E}-02$ & $5.12 \mathrm{E}-01$ & $2.06 \mathrm{E}+00$ \\
\hline${ }^{241} \mathrm{Pu}^{(f)}$ & 8.82E-01 & $5.38 \mathrm{E}-01$ & $3.36 \mathrm{E}-01$ & $8.51 E-01$ & $3.43 E+00$ & $2.55 E+01$ & $1.03 E+02$ \\
\hline${ }^{242} \mathrm{Pu}^{(\mathrm{g})}$ & $2.96 \mathrm{E}-05$ & 1.81E-05 & $1.13 \mathrm{E}-05$ & $2.85 E-05$ & $1.15 \mathrm{E}-04$ & $8.56 \mathrm{E}-04$ & $3.45 \mathrm{E}-03$ \\
\hline${ }^{241} \mathrm{Am}^{(\mathrm{a}, \mathrm{h})}$ & $4.01 E-02$ & $2.06 E-01$ & $5.57 \mathrm{E}-02$ & 1.57E-01 & $7.57 \mathrm{E}-01$ & $4.72 E+00$ & $2.27 E+01$ \\
\hline${ }^{243} \mathrm{Cm}^{(0)}$ & $1.59 E-04$ & $2.75 E-03$ & $9.78 \mathrm{E}-04$ & $3.84 E-03$ & $2.20 \mathrm{E}-02$ & $1.15 E-01$ & $6.61 \mathrm{E}-01$ \\
\hline${ }^{244} \mathrm{Cm}^{(\mathrm{k})}$ & $1.09 \mathrm{E}-02$ & $1.89 \mathrm{E}-01$ & $6.74 \mathrm{E}-02$ & $2.65 E-01$ & $1.52 E+00$ & $7.94 \mathrm{E}+00$ & $4.55 \mathrm{E}+01$ \\
\hline${ }^{79} \mathrm{Se}^{(\mathrm{m})}$ & 9.46E-06 & 4.87E-05 & 1.32E-05 & $3.71 \mathrm{E}-05$ & $1.79 \mathrm{E}-04$ & $1.11 E-03$ & $5.36 \mathrm{E}-03$ \\
\hline${ }^{99} \mathrm{Tc}^{(\mathrm{n})}$ & $3.14 \mathrm{E}-04$ & $1.61 \mathrm{E}-03$ & $4.36 E-04$ & $1.23 \mathrm{E}-03$ & $5.93 E-03$ & $3.70 \mathrm{E}-02$ & $1.78 \mathrm{E}-01$ \\
\hline
\end{tabular}

Assumptions: (a) measured activity used, if available

(b) ${ }^{90} \mathrm{Sr}={ }^{137} \mathrm{Cs} \times 6.00 \mathrm{E}-01$

(c) ${ }^{238} \mathrm{Pu}={ }^{238} \mathrm{Pu} /{ }^{241} \mathrm{Am} \times 1.85 \mathrm{E}-01$
(d) ${ }^{239} \mathrm{Pu}={ }^{239 / 240} \mathrm{Pu} \times 5.05 \mathrm{E}-01$
(e) ${ }^{240} \mathrm{Pu}={ }^{239 / 240} \mathrm{Pu} \times 4.95 \mathrm{E}-01$
(f) ${ }^{241} \mathrm{Pu}={ }^{239 / 240} \mathrm{Pu} \times 2.47 \mathrm{E}+01$
(g) ${ }^{242} \mathrm{Pu}={ }^{239 / 240} \mathrm{Pu} \times 8.28 \mathrm{E}-04$

(h) ${ }^{241} \mathrm{Am}={ }^{238} \mathrm{Pu} /{ }^{241} \mathrm{Am} \times 8.15 \mathrm{E}-01$

(j) ${ }^{243} \mathrm{Cm}={ }^{243 / 244} \mathrm{Cm} \times 1.43 \mathrm{E}-02$

(k) ${ }^{244} \mathrm{Cm}={ }^{243 / 244} \mathrm{Cm} \times 9.86 \mathrm{E}-01$

(m) ${ }^{79} \mathrm{Se}={ }^{241} \mathrm{Am} \times 2.36 \mathrm{E}-04$

(n) ${ }^{99} \mathrm{Tc}={ }^{241} \mathrm{Am} \times 7.83 \mathrm{E}-03$

(p) total $=$ removable $\times 3.00 \mathrm{E}+01$ 
WCH-412

Rev. 0

\section{APPENDIX B}

\section{RADIOLOGICAL SURVEY REPORTS}


PHMC M2572, Contamination Survey for Facility Characterization, dated 02/03/98 ............. B-1

PHMC M2781, Characterization Survey of C-Cell, dated 02/25/98 .................................... B-5

PHMC M2793, Smears Taken for Characterization of A-Cell; 24-hr Count of C-Cell Smears, dated $02 / 25 / 98$

PHMC M2809, Characterization Smears of D-Cell, SMF Airlock, SMF East Cell, and 24-Hr Count on D-Cell Smear, dated 02/26/98.

PHMC M2818, B-Cell Smears and 24-Hr Count of Smears of D-Cell, SMF Airlock, and SMF East Cell, dated 02/27/98

PHMC M2873, Dose Rate and Contamination Level Survey of Smears from B/D-Cells, dated 03/04/98

PHMC M2896, Contamination Level Survey of C-Cell Smears, dated 03/05/98................. B-17

PHMC M2966, Smears Taken in REC Airlock, dated 03/11/98 ....................................... B-19

PHMC M2982, Contamination Survey of Characterization Samples, dated 03/12/98........... B-21

PHMC M2991, Contamination Level Survey of SMF Characterization Samples, dated

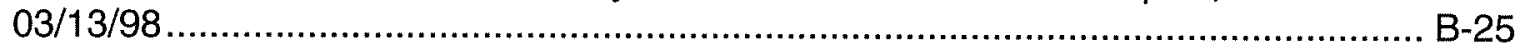

PHMC M3014, 72-Hr Survey of Characterization Smears from SMF South Cell \& Compartment 1 , dated 03/16/98. 


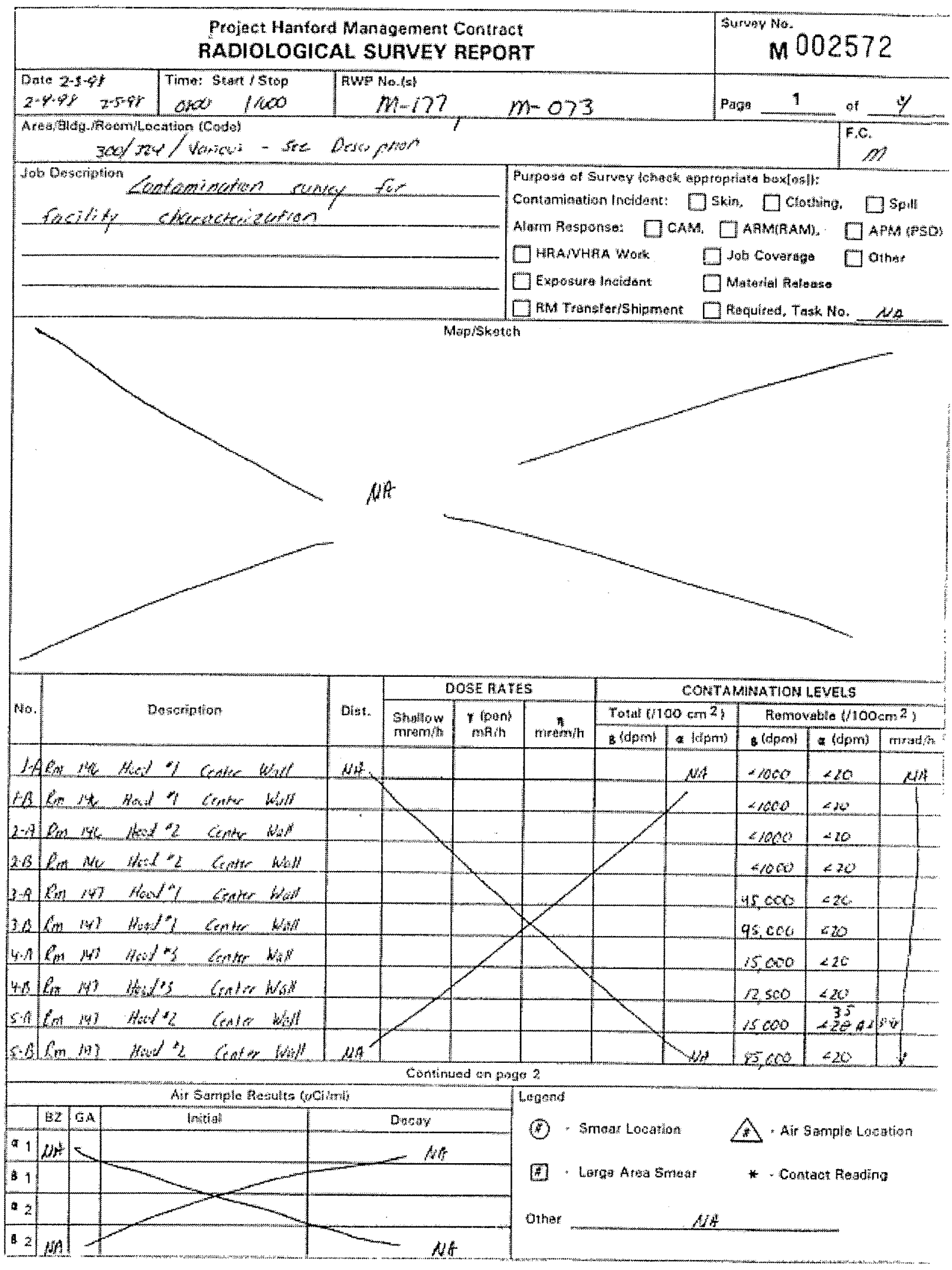

80.00001910201 


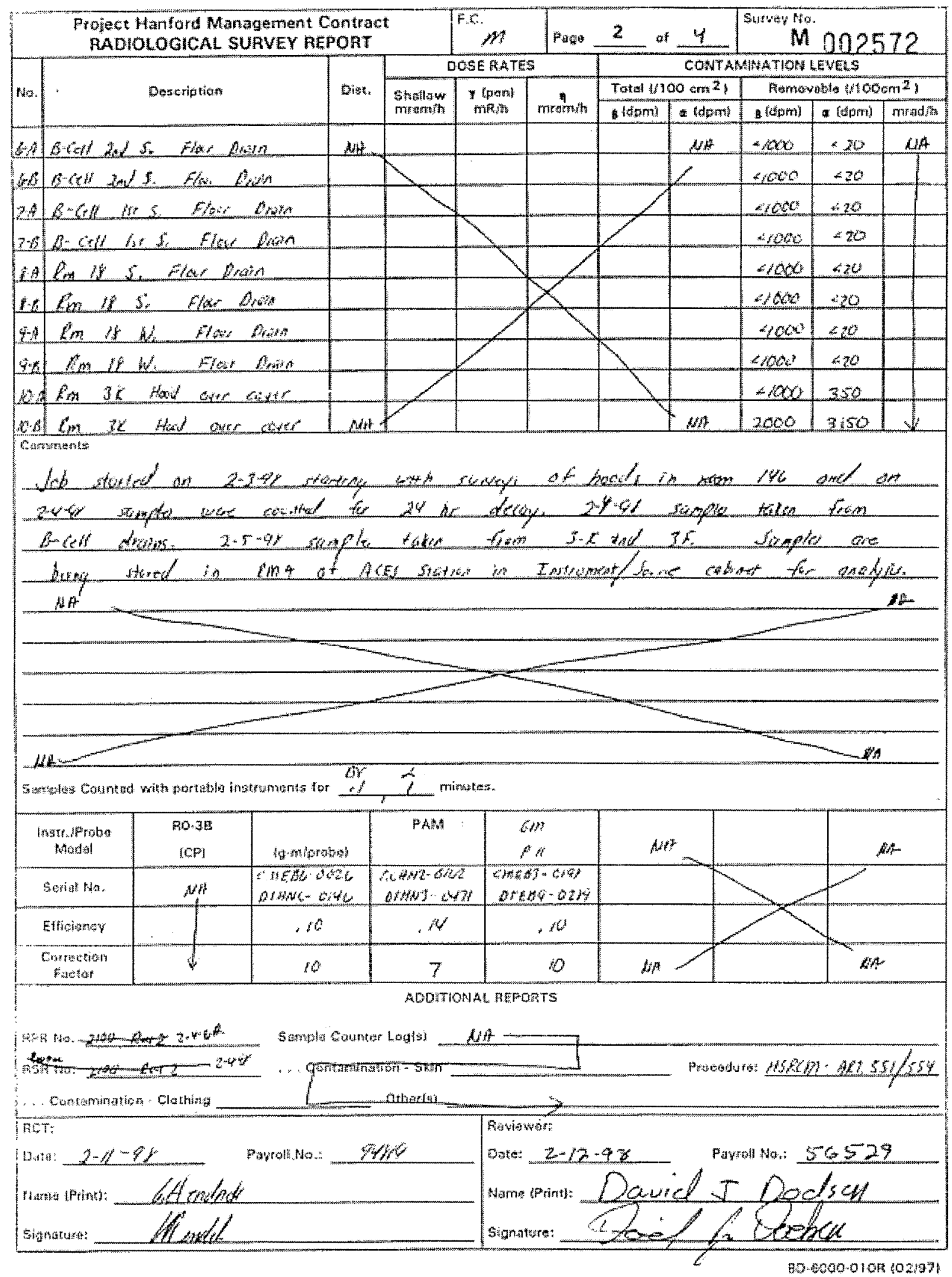




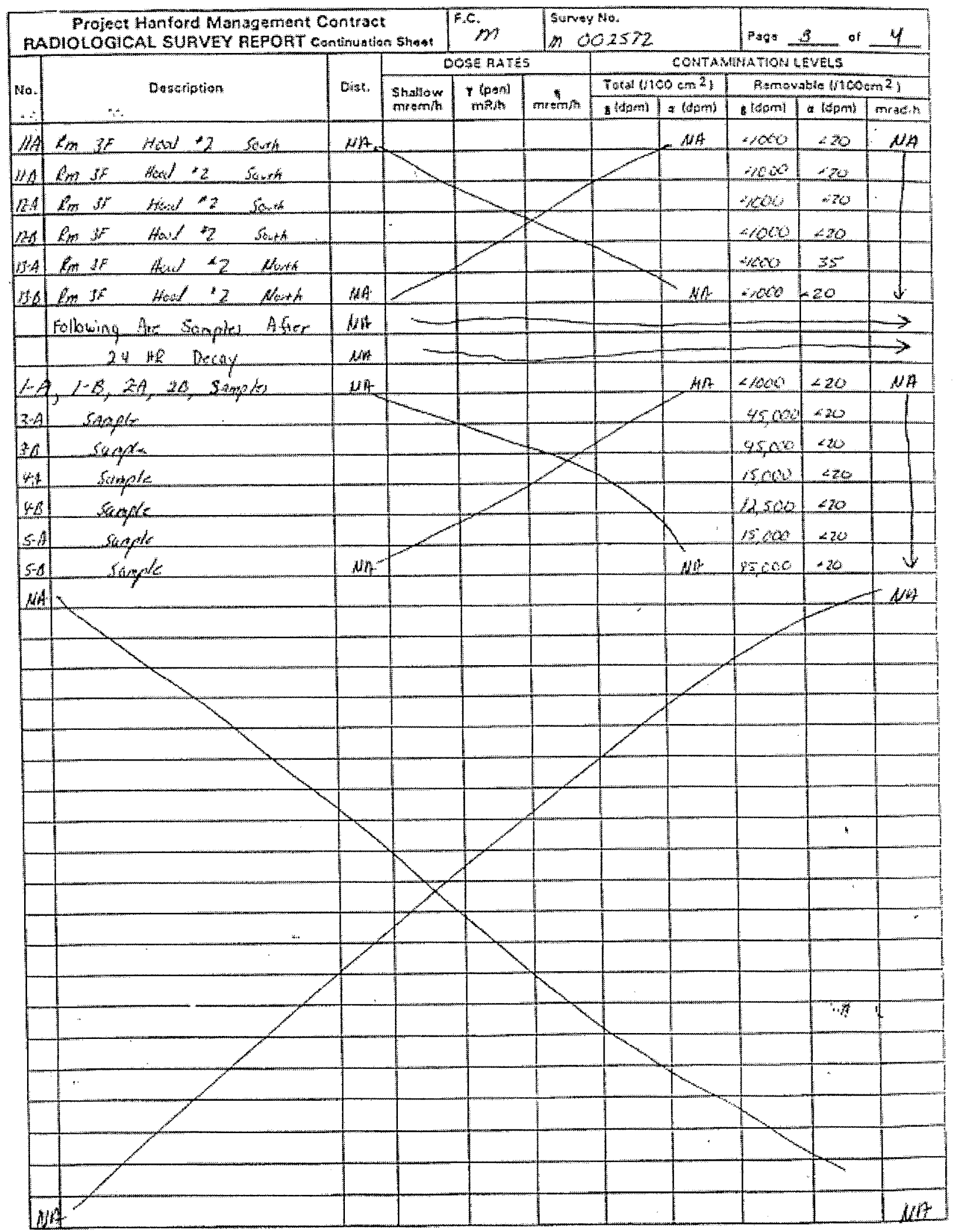

8D.6000.010,1 10297? 


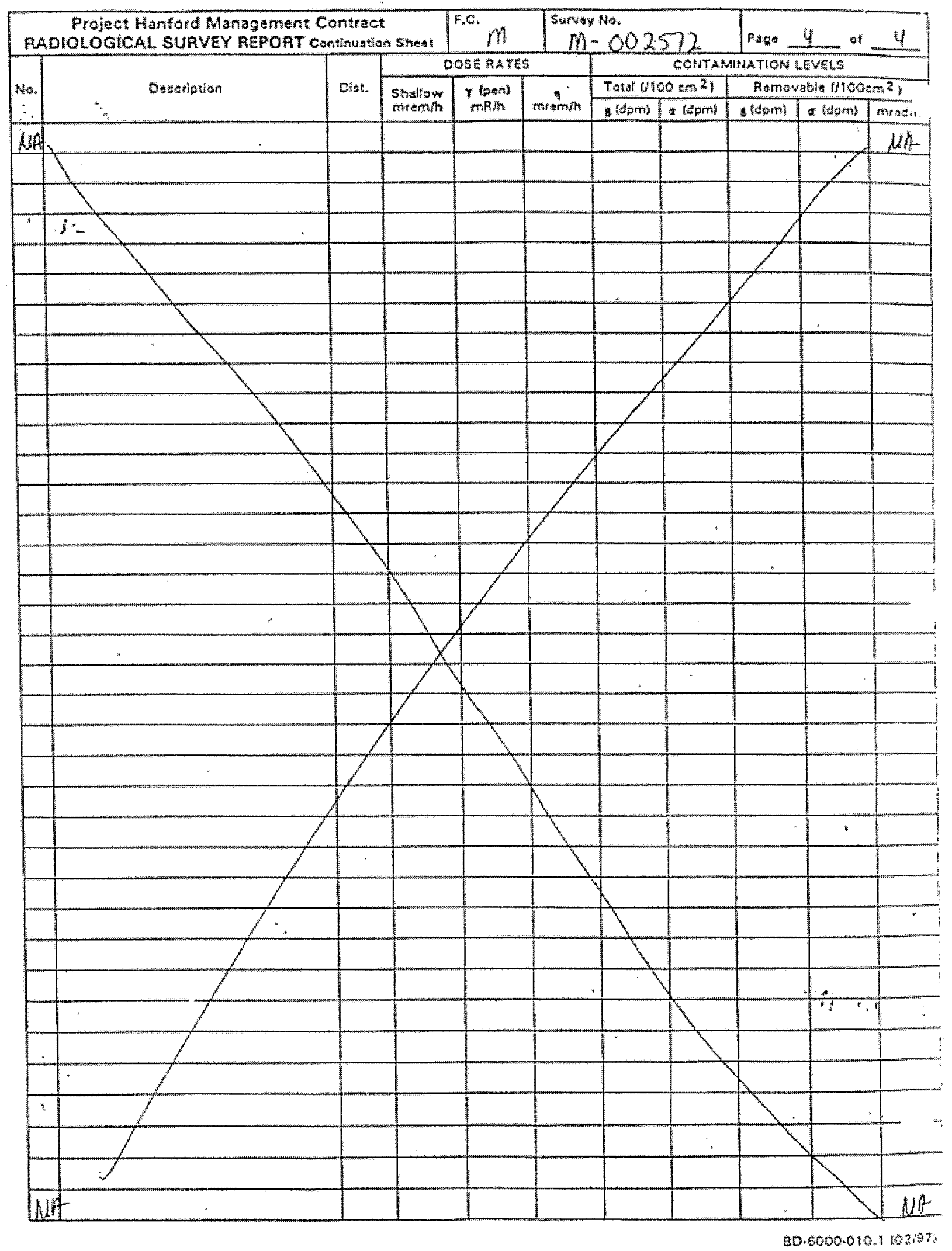




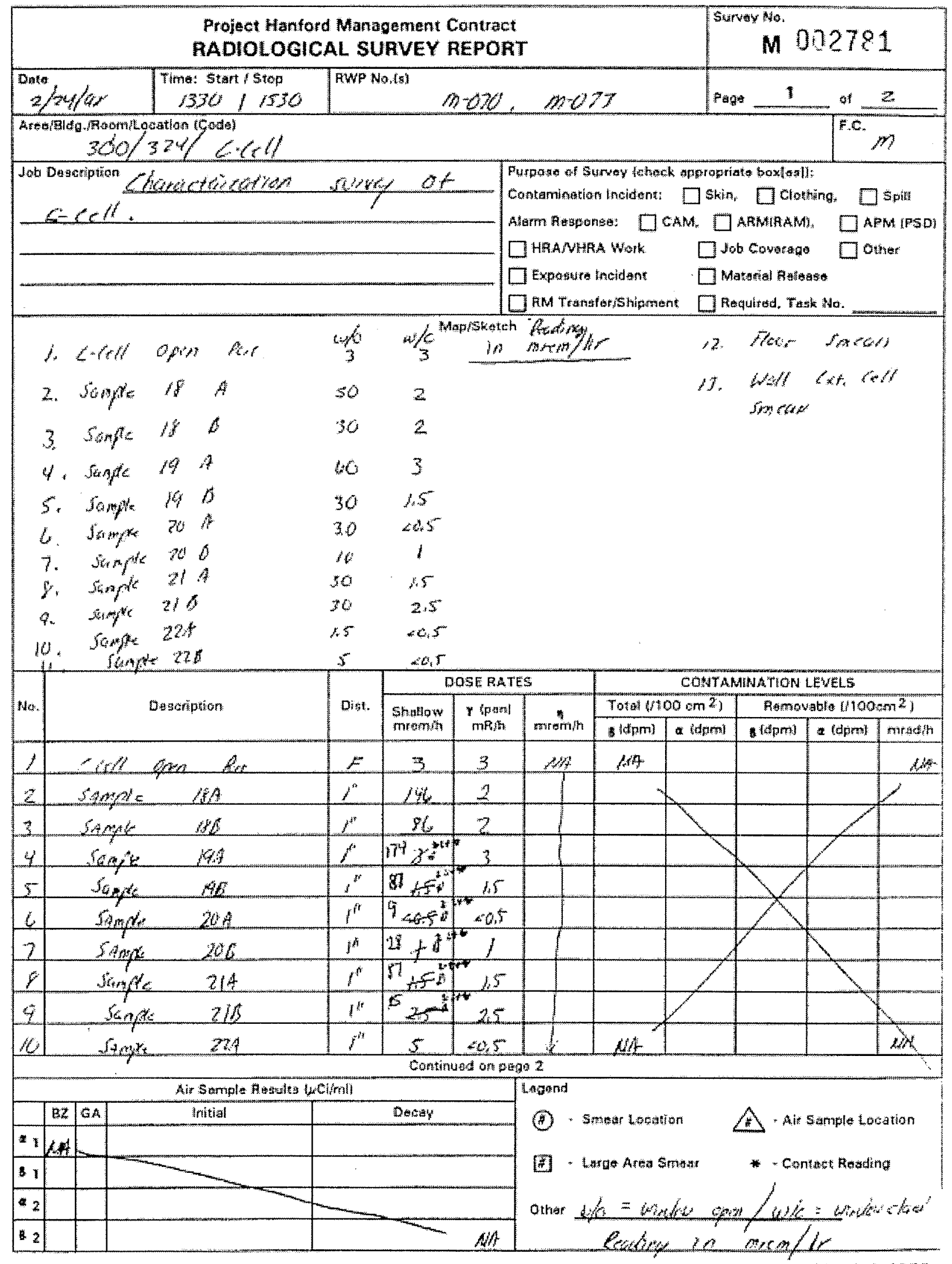




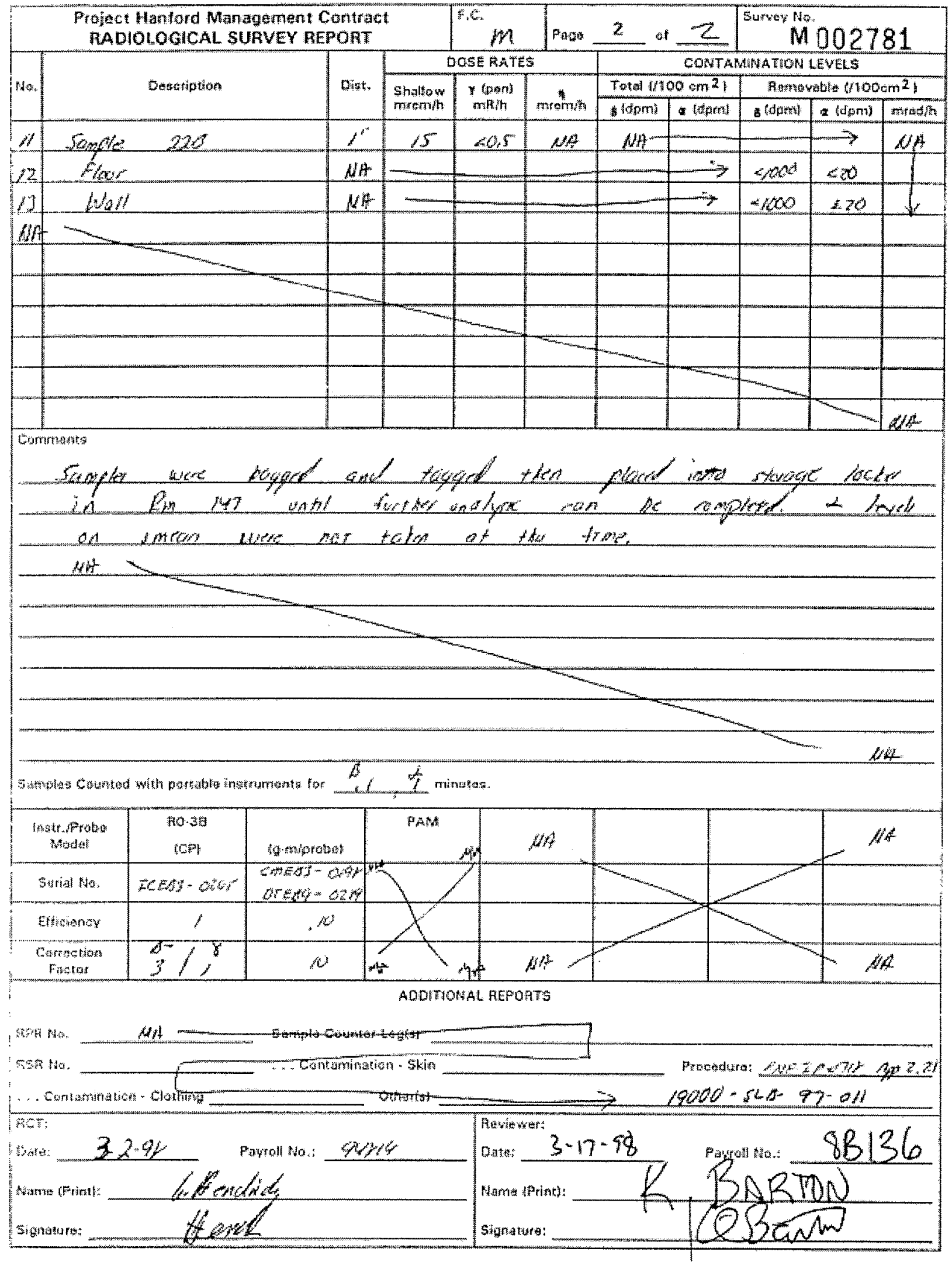

ED-8000010R 602397 


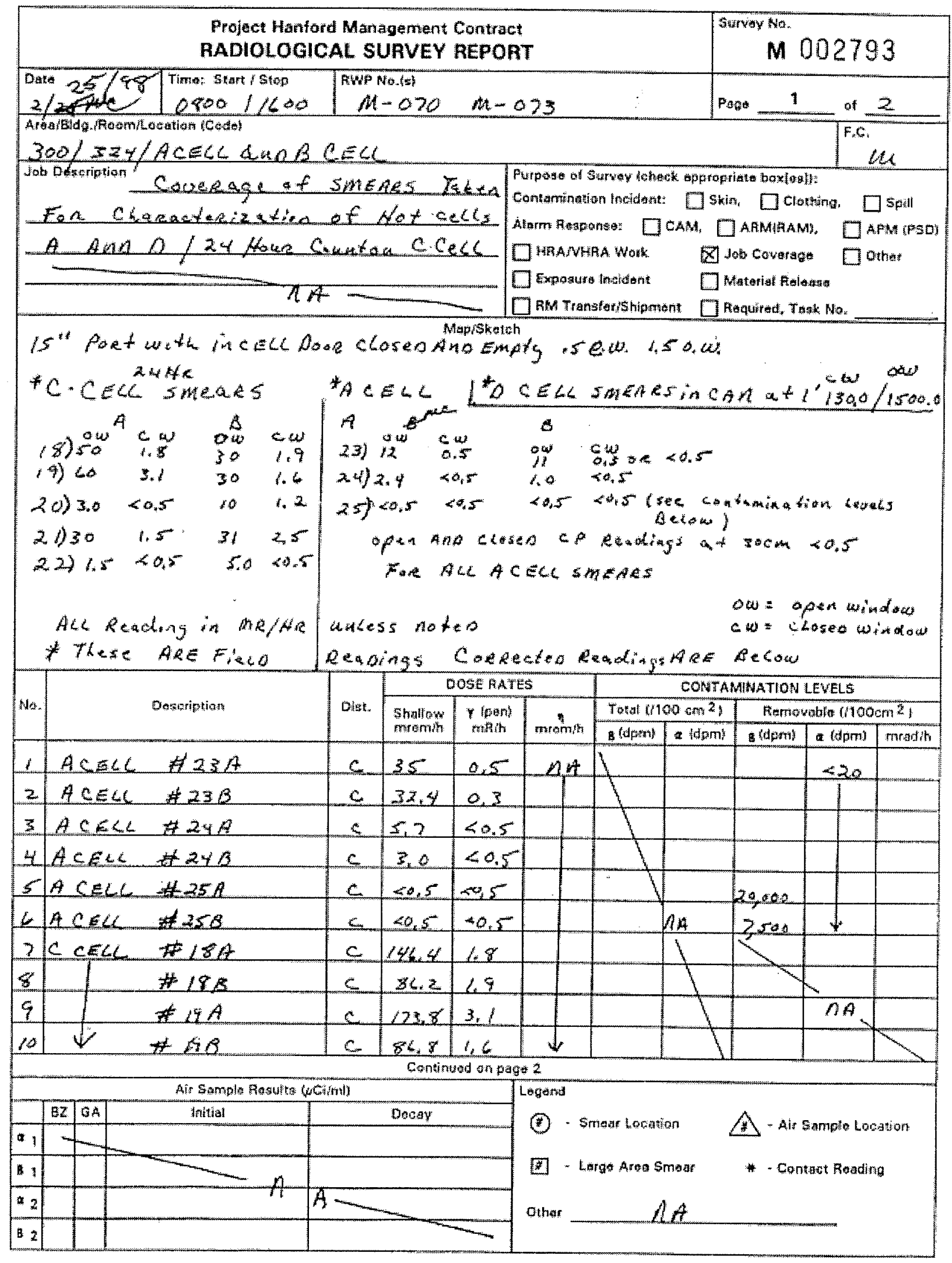

00.6000 .01010297 


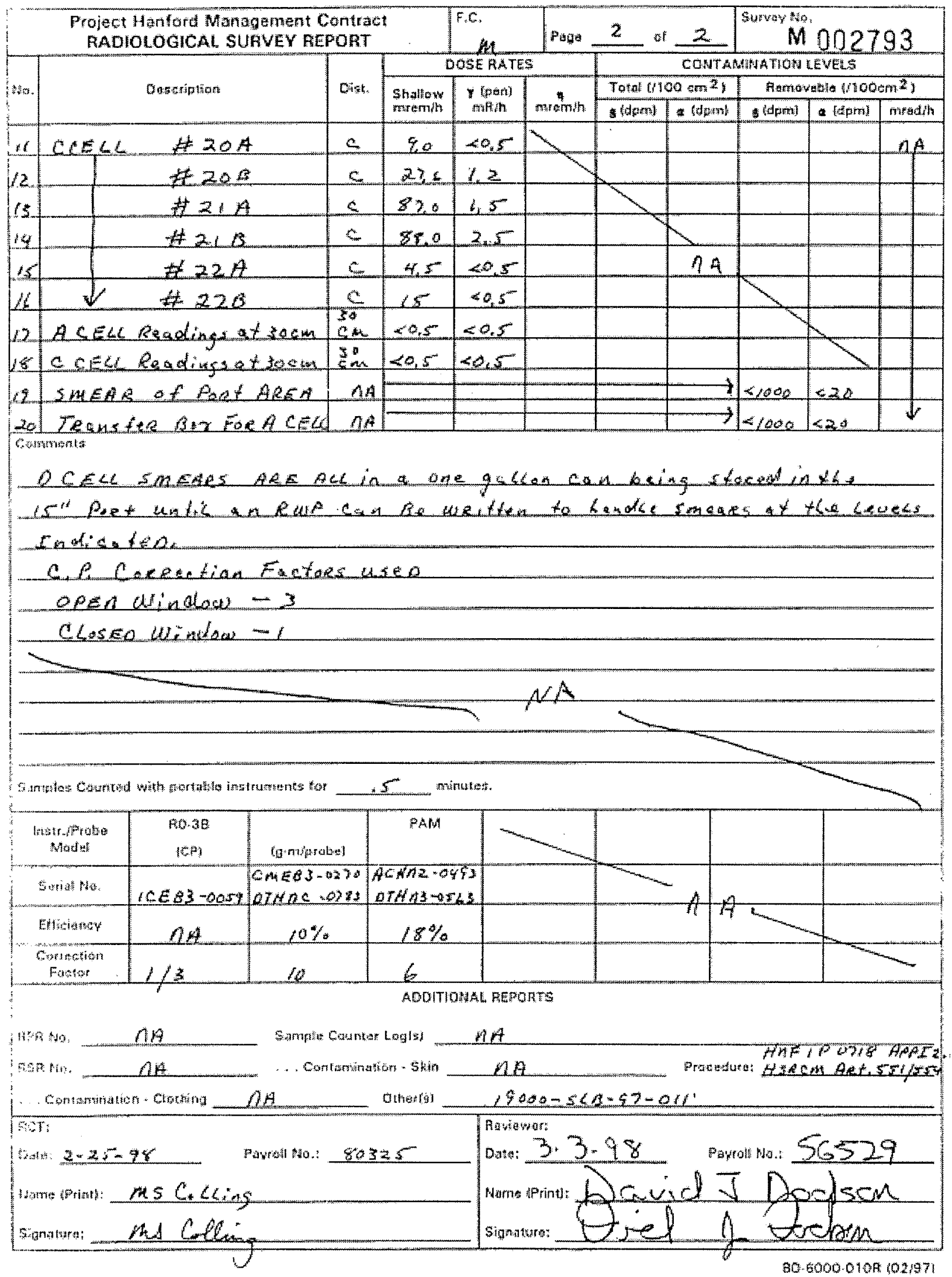




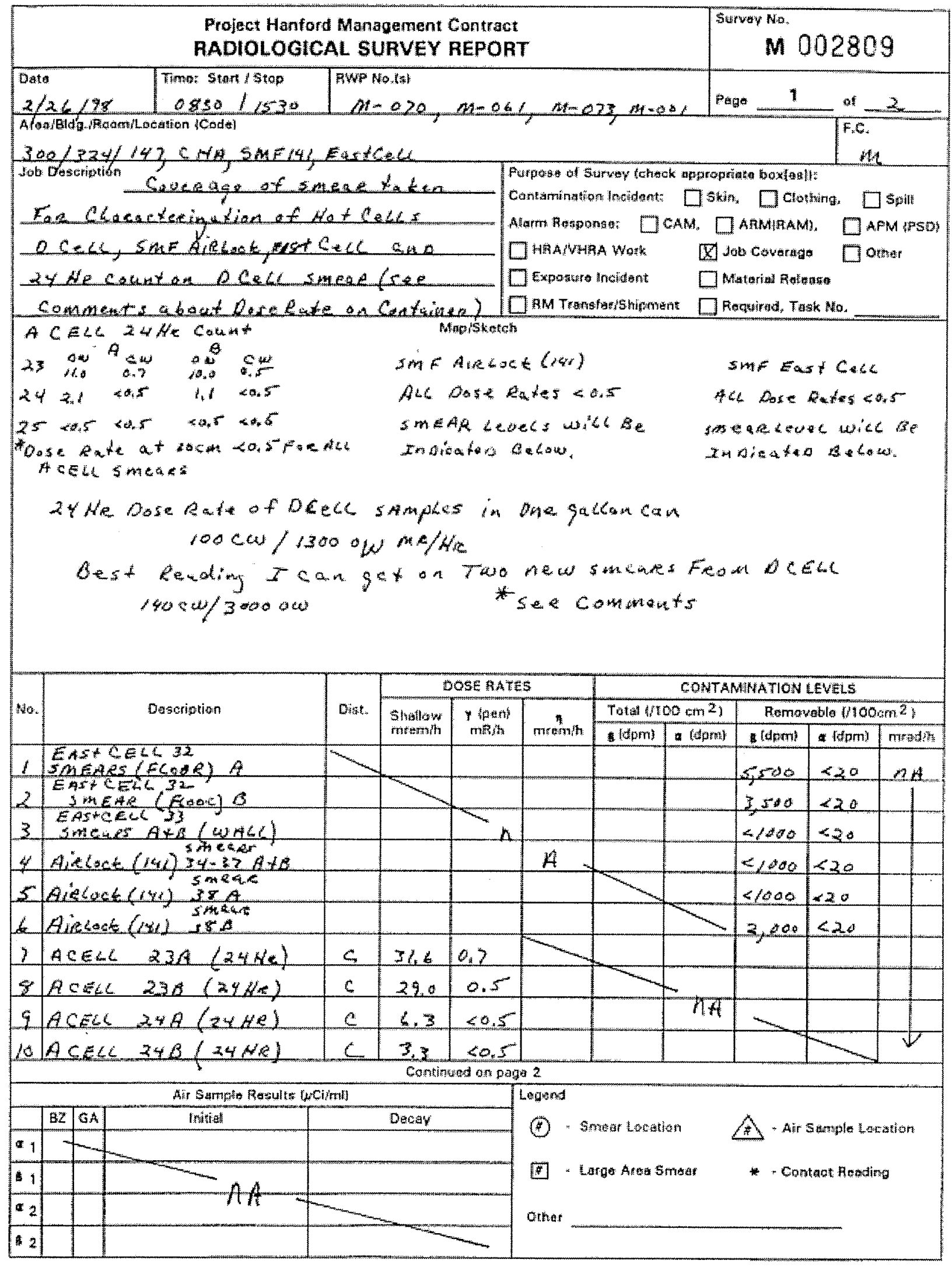

80.6000010102397 


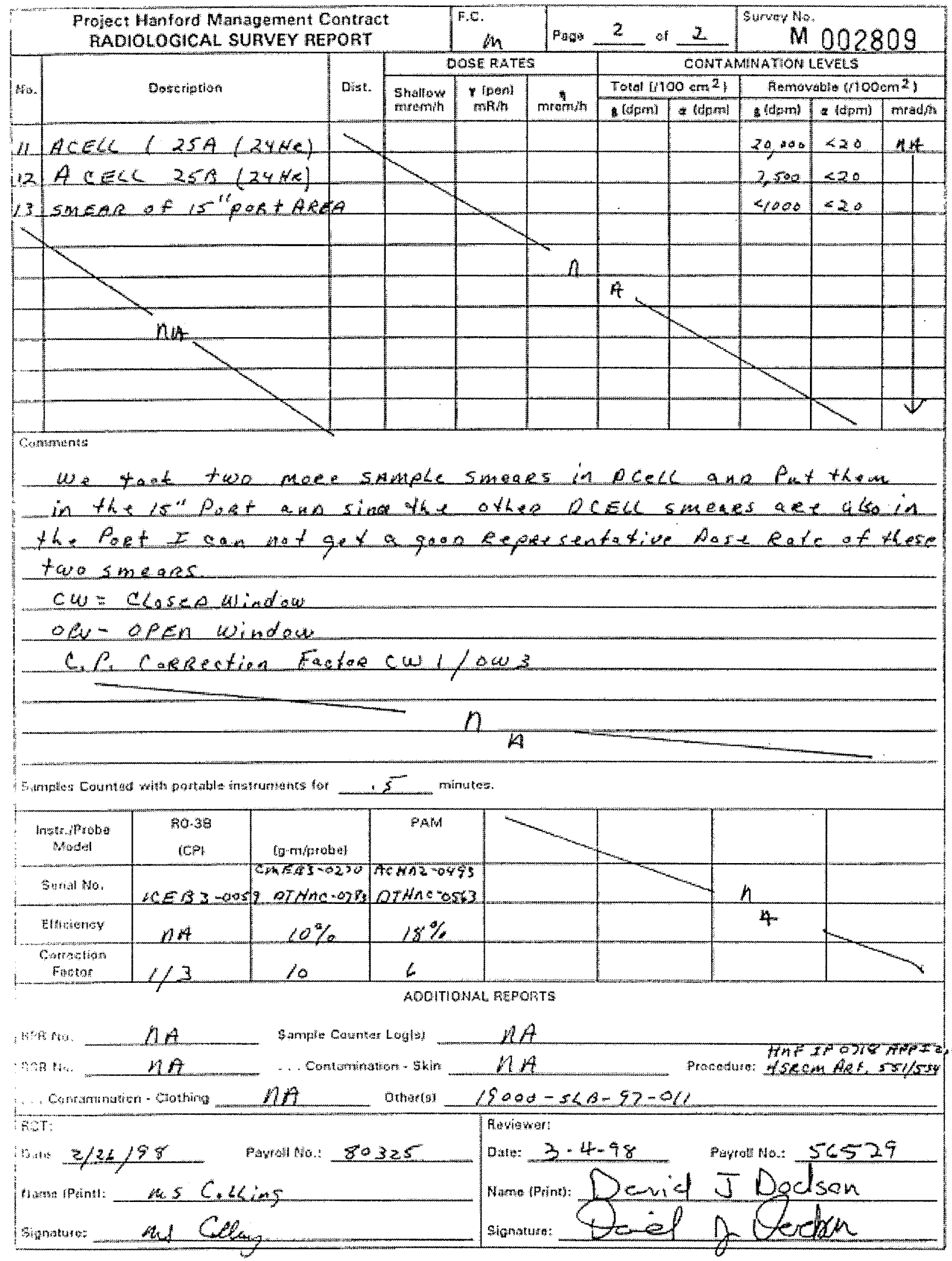

60 $6000.010 \mathrm{H} 10297$ 


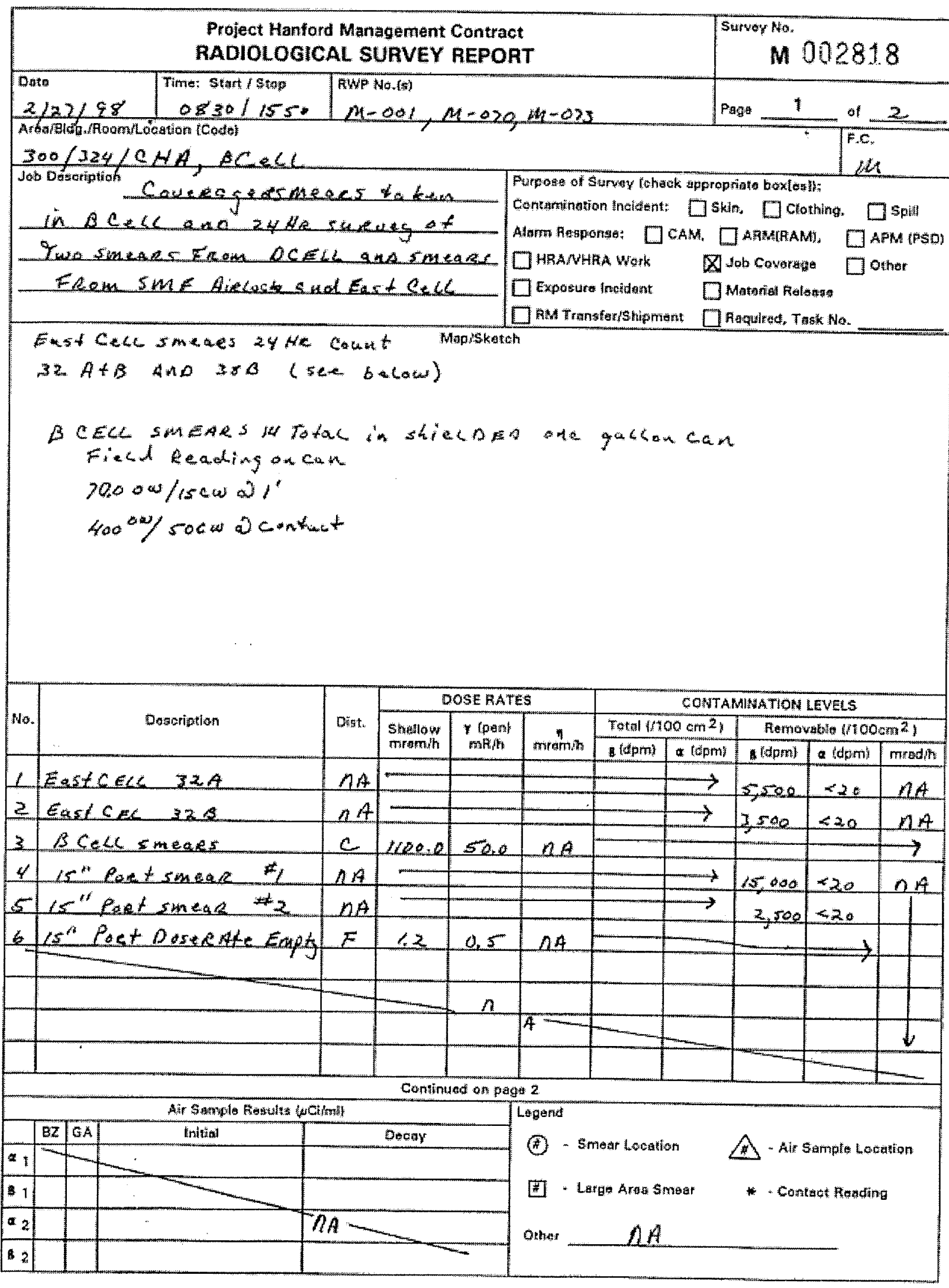




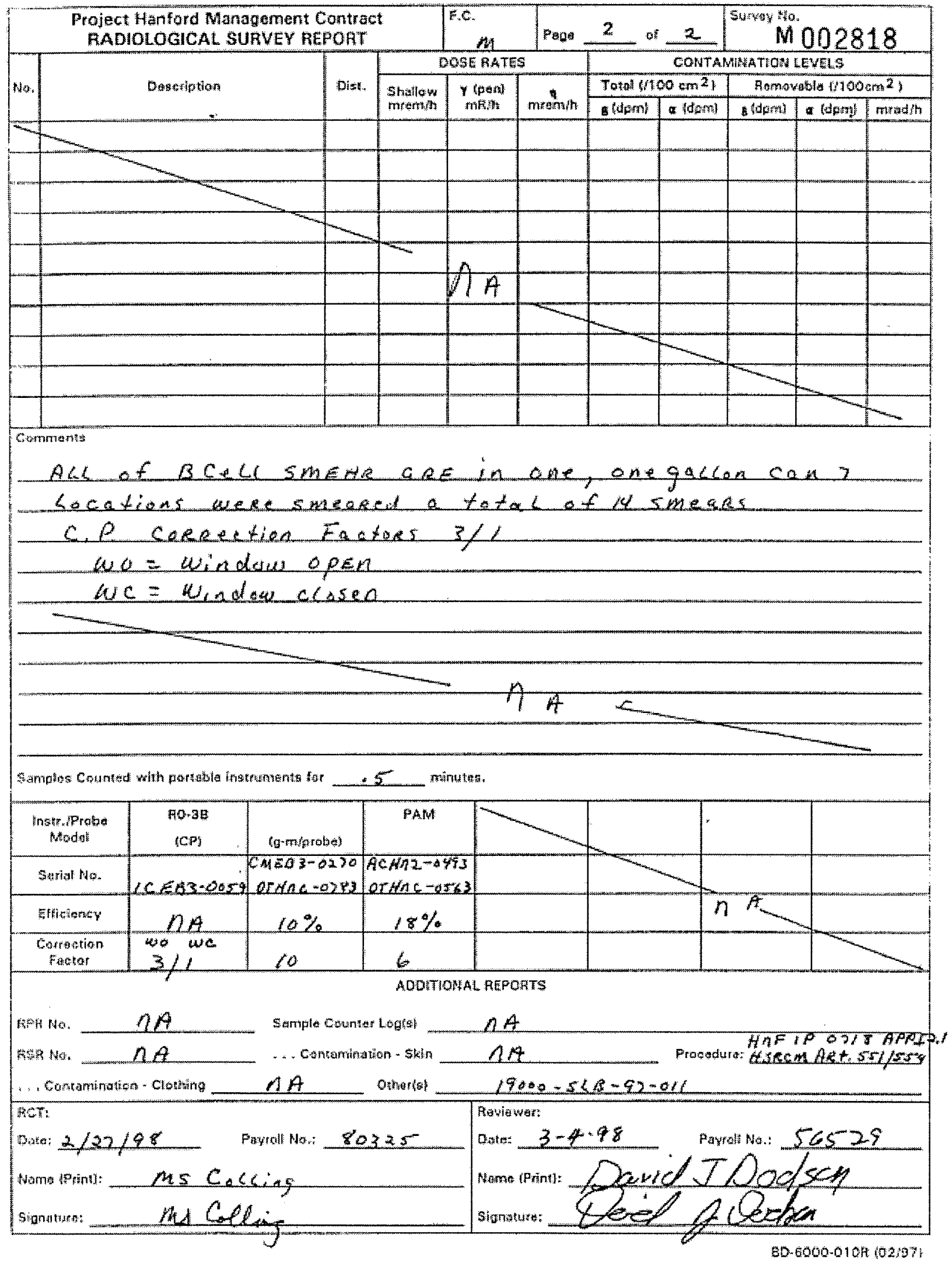




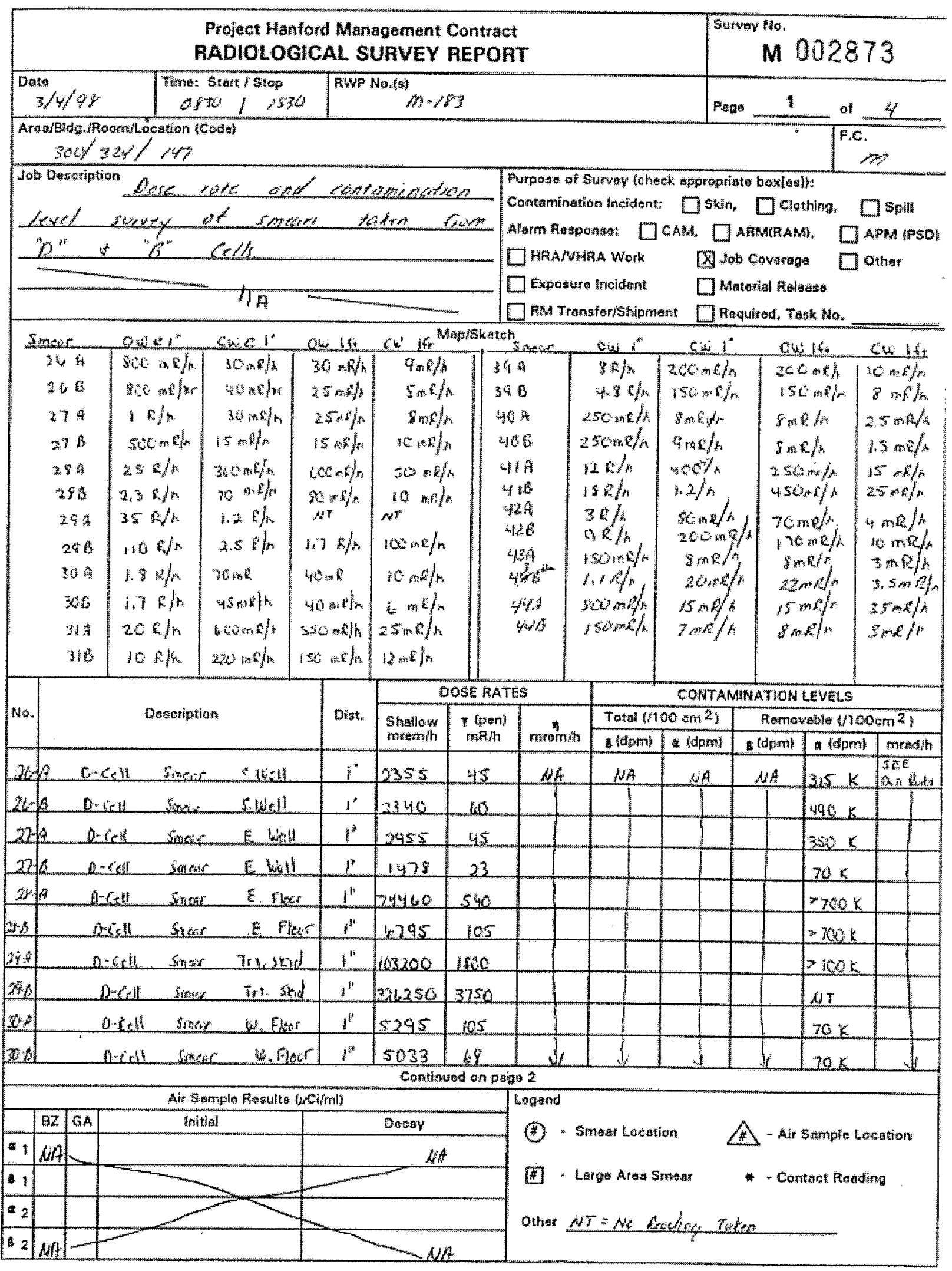

00-6000-010 10297 


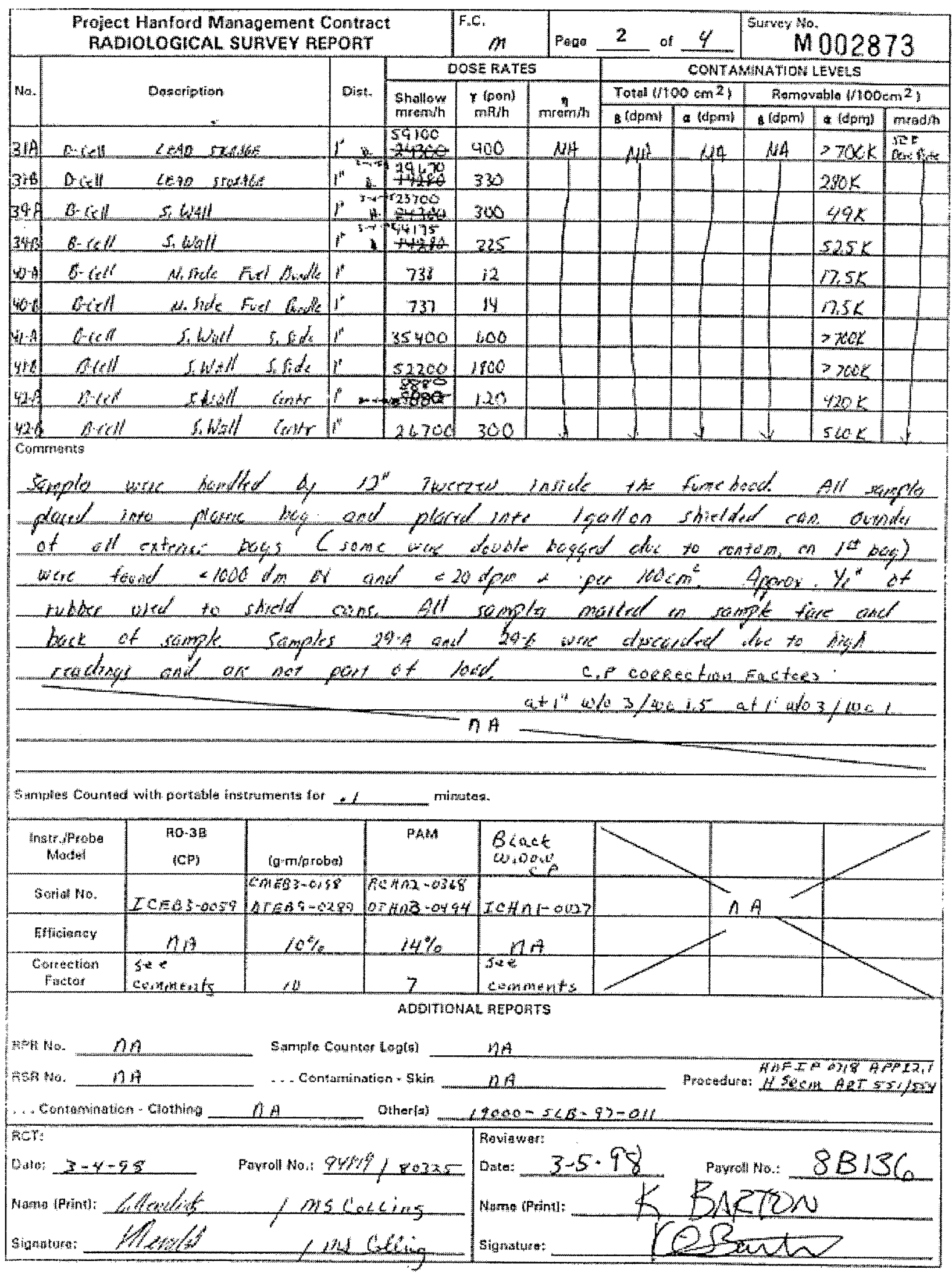

B0.6000010\% 6028\% 


\begin{tabular}{|c|c|c|c|c|c|c|c|c|c|c|}
\hline \multicolumn{4}{|c|}{$\begin{array}{l}\text { Project Hanford Marnagement Contract } \\
\text { RADIOLOGICAL SURVEY REPORT Continuation Sheot }\end{array}$} & $F_{0}$ & \multicolumn{3}{|c|}{ Survay No. } & \multicolumn{3}{|c|}{ Paga 3 of 4} \\
\hline \multirow{3}{*}{ No. } & \multirow{3}{*}{ Description } & \multirow{3}{*}{ bist. } & \multicolumn{3}{|c|}{ DOSE RATES } & \multicolumn{5}{|c|}{ CONTAMINAMTION LEVELS } \\
\hline & & & \multirow{2}{*}{$\begin{array}{l}\text { stallow } \\
\text { miremper }\end{array}$} & \multirow{2}{*}{$\begin{array}{c}x \text { toen } \\
m R / h\end{array}$} & \multirow{2}{*}{ mormo } & \multicolumn{2}{|c|}{ Foral $1100 \operatorname{sen}^{2}$ ? } & \multicolumn{3}{|c|}{ Femovybto (hoogem ${ }^{2}$ ) } \\
\hline & & & & & & Eidpenl & a (dem) & Etdpen & * Adporit & mraadin \\
\hline$\forall x$ & 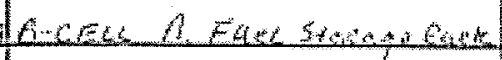 & $f^{n}$ & $4=8$ & 2 & $\mathrm{AA}$ & $\Delta A$ & $\Delta a$ & $A B$ & $24 k$ & $\hat{b e x}^{2}$ \\
\hline 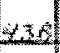 & 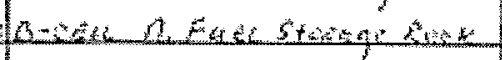 & $i^{*}$ & 230 & $3 s^{4}$ & & 1 & 1 & $\therefore$ & $2 x k$ & x \\
\hline twas & 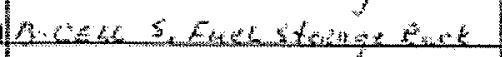 & $t^{\prime \prime}$ & $232 \%$ & 23 & & & & & $41 k$ & \\
\hline 40 & 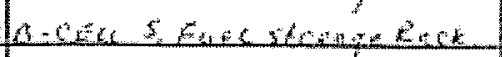 & $t^{*}$ & 푸이 & 3 & & & & & $2, k$ & \\
\hline 20 & 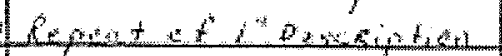 & $y^{\prime \prime}$ & 22 & 9 & & & & & 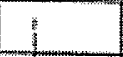 & \\
\hline 20.6 & & $2^{4}$ & 52 & 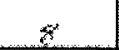 & & & & & & \\
\hline Bin & & $12^{10}$ & 5,3 & 5 & & & & & & \\
\hline 200 & & 2 & 25 & 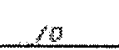 & & & & & & \\
\hline sal & & $12^{*}$ & 20. & 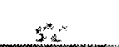 & & & & & & \\
\hline ind & & $x^{x y}$ & $2 \lambda t^{2}$ & $\infty$ & & & & & & \\
\hline ate & & $1 z^{\prime \prime}$ & $8_{3,2}$ & 1200 & & & & & & \\
\hline 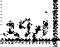 & & $x^{\circ}$ & $a r$ & $2 \pi$ & & & & & & \\
\hline ( & & $12^{\prime \prime}$ & 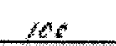 & $s$ & & & & & & \\
\hline $\sin d$ & & 2 & $10 s^{\prime}$ & \pm & & & & & & \\
\hline 204 & & $2 x^{\circ}$ & stes & 25 & & & & & & \\
\hline 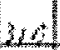 & & $2^{*}$ & +2 & $i^{3}$ & & & & & & \\
\hline 37, & & $x 7^{\circ}$ & sio & $\omega$ & & & & & & \\
\hline 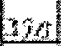 & & $x^{n}$ & 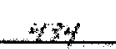 & 8 & & & & & & \\
\hline 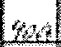 & & $2^{4}$ & 19 & 25 & & & & & & \\
\hline vers & & $+2^{i 4}$ & 26 & 15 & & & & & & \\
\hline ty,al & & $12^{\prime \prime}$ & MAC: & $25^{\circ}$ & & & & & & \\
\hline wino & & $y^{*}$ & 22 & $.5^{\circ}$ & & & & & & \\
\hline sag & & $z^{\prime \prime}$ & $2 g^{2}$ & 4 & & & & & & \\
\hline 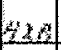 & & $12^{n}$ & $49 \mathrm{c}$ & 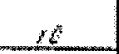 & & & & & ' & \\
\hline 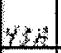 & & $12^{2}$ & $1 s$ & 3 & & & & & & \\
\hline$(x)$ & & $12^{4}$ & $5 \%$ & 3.5 & & & & & & \\
\hline vita & & $1 x^{n}$ & 30 & 35 & & & & & & " \\
\hline $4+1$ & & $12^{\prime \prime}$ & 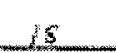 & 3 & & & & & & \\
\hline $4 a$ & & $12^{\prime \prime}$ & 76 & 6 & & & & & & \\
\hline$y_{503}$ & $\downarrow$ & $z^{\prime \prime}$ & 670 & 10 & & & & & & \\
\hline & Max Exteruet Bore Rate & $4^{n}$ & 11260 & 120 & & & & & & \\
\hline & 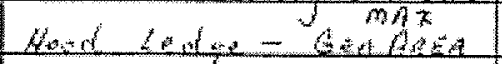 & $F$ & 265 & 15 & & & & 4 & $\sqrt{ }$ & \\
\hline & Hoon Ledee - sencous & $D A$ & & $\longrightarrow$ & & & & rines & $<20^{\circ}$ & \\
\hline & Etone sutentes & $n+4$ & & $m$ & & & & $<\theta^{0}$ & $<20$ & \\
\hline & 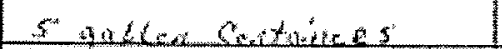 & $6^{.7}$ & 35 & 15 & & & & $<160$ & $=20$ & \\
\hline $\mid 150$ & 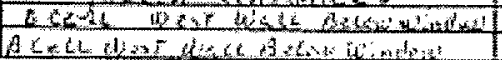 & $r^{\circ}$ & $\frac{7.3}{630}$ & $\frac{-6}{10}$ & $\downarrow$ & $\downarrow$ & T & $\frac{n \theta^{2}}{n t^{2}}$ & $\frac{140 E}{2010}$ & $\downarrow$ \\
\hline
\end{tabular}




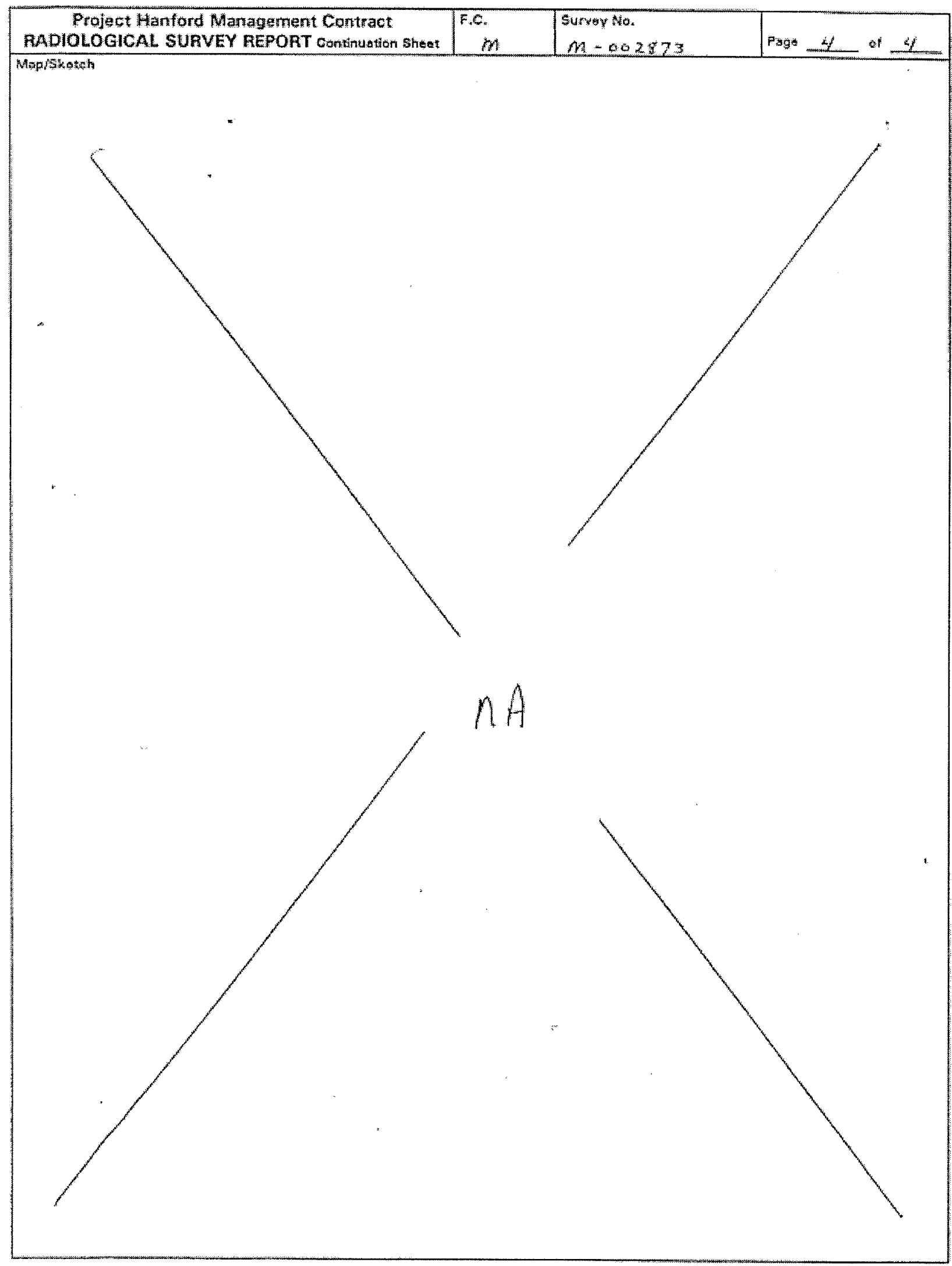

a0. 5000.010.1010207\% 


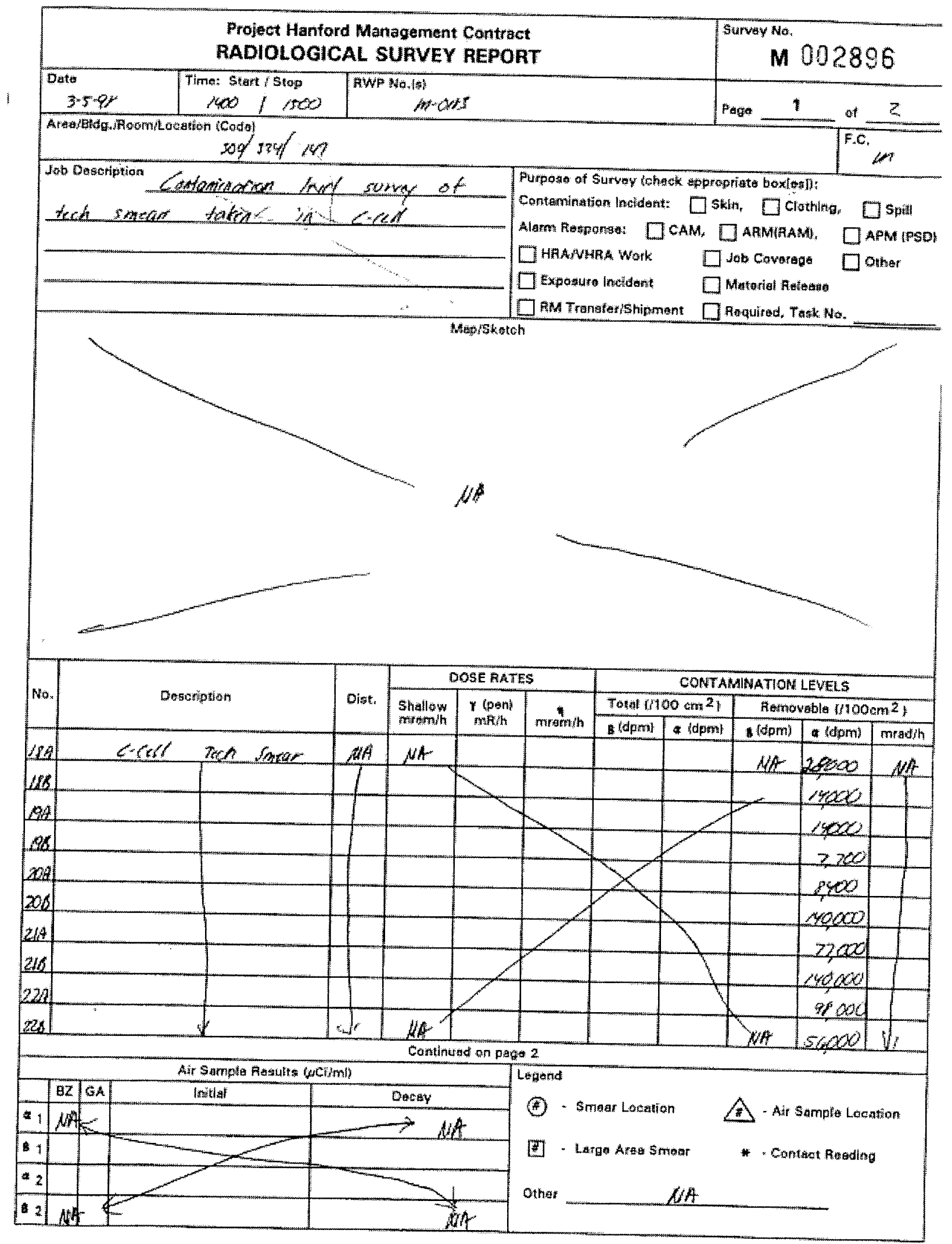

$80 \cdot 6000-010102197)$ 


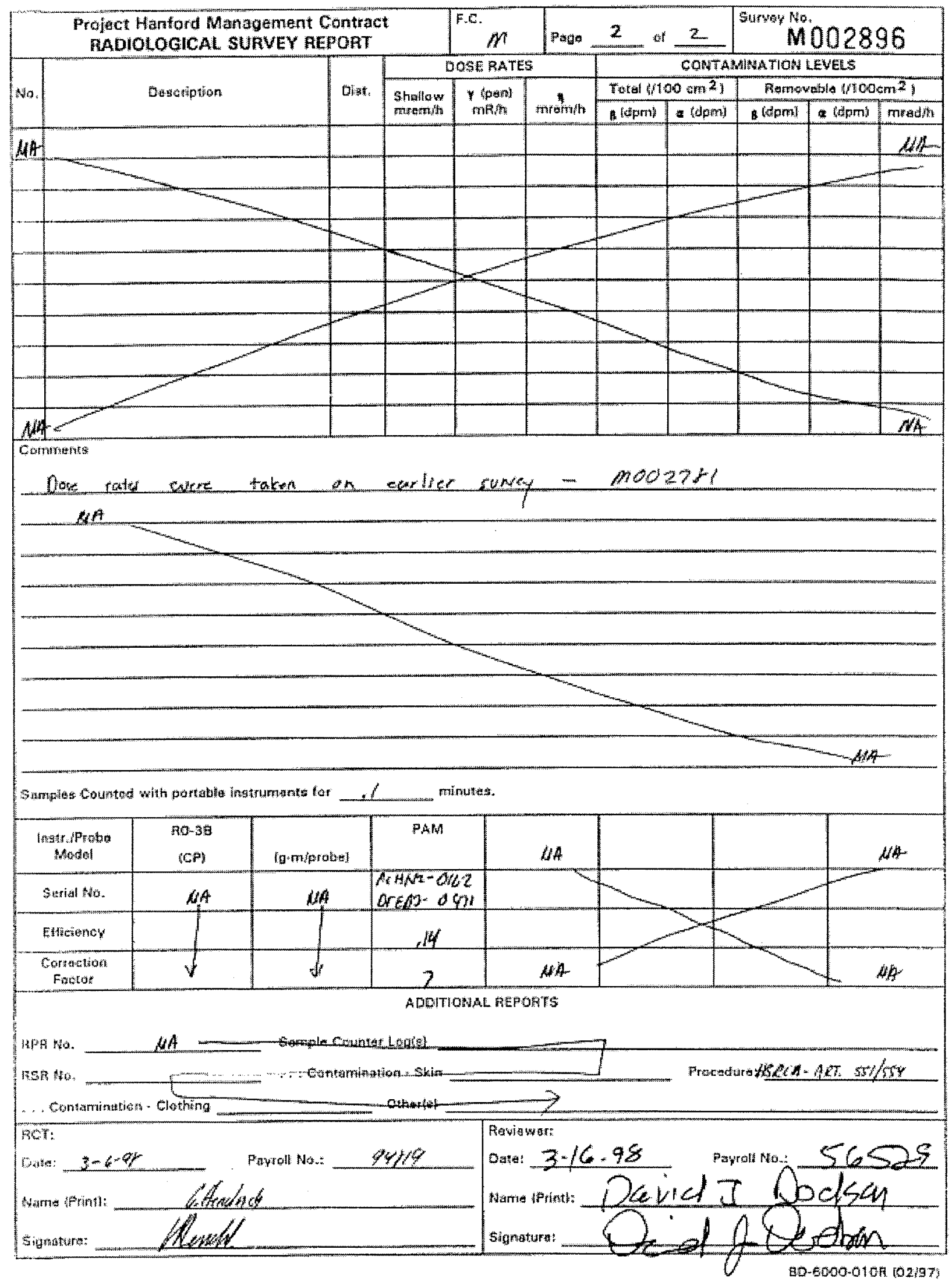




\begin{tabular}{|c|c|c|c|c|c|c|c|c|c|c|c|c|}
\hline \multicolumn{10}{|c|}{$\begin{array}{l}\text { Project Hanford Management Contract } \\
\text { RADIOLOGICAL. SURVEY REPORT }\end{array}$} & \multicolumn{3}{|c|}{ M 002966} \\
\hline \multicolumn{3}{|c|}{$3 / 1468$} & {$\left[\begin{array}{l}\text { Tims; stert / Stap } \\
0 \text { soo } / / 600\end{array}\right.$} & \multicolumn{6}{|c|}{$\begin{array}{r}\text { RWP No.Is) } \\
M-183\end{array}$} & Page 1 & & of \\
\hline \multicolumn{11}{|c|}{ 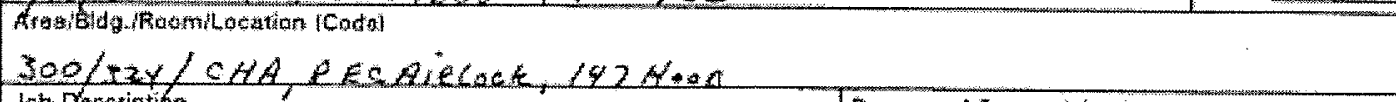 } & \multicolumn{2}{|c|}{ Fic. } \\
\hline \multicolumn{7}{|c|}{ 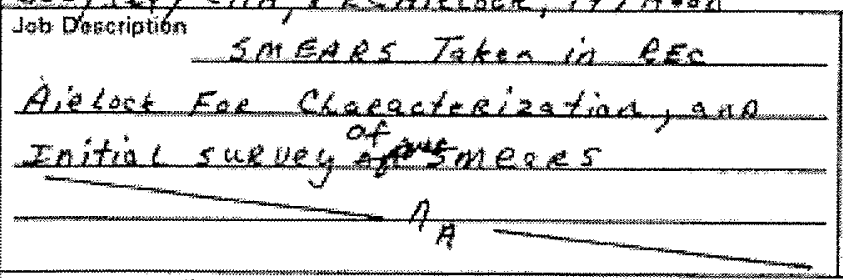 } & \multicolumn{6}{|c|}{ 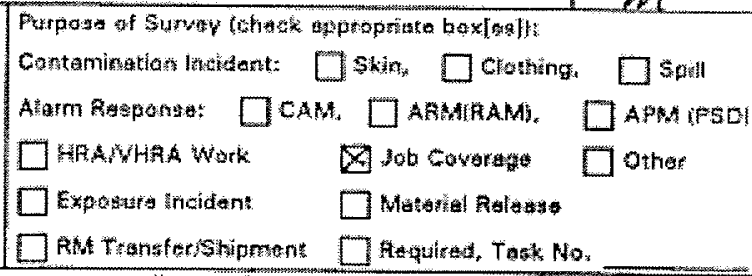 } \\
\hline \multirow{6}{*}{\multicolumn{2}{|c|}{$\begin{array}{l}63 A \\
43 B \\
64 A \\
44 A \\
45 A\end{array}$}} & & Wo 140 & & & aprisketoh & $i^{\prime \prime}$ & $\omega / 0$ & & $12^{7}$ wot & & \\
\hline & & & $17 \quad-12$ & & & 23.4 & & 4 & & .5 & & \\
\hline & & & 1 & & & 636 & & 2 & & .5 & & \\
\hline & & & 3 & & & $\Delta \psi^{2}$ & . & & & -2 & & \\
\hline & & & $-1 s^{m}$ & & & $6+\pi$ & - & 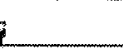 & & 1 & & \\
\hline & & & $2 k$ & & & $45 A$ & 1 & $\hat{z}$ & & 1.2 & & \\
\hline \multirow{2}{*}{\multicolumn{2}{|c|}{656}} & & 960 & & & 250 & 1 & s & & 14 & & \\
\hline & & & 格新 & & & 204 & A & $s$ & & +7 & & \\
\hline \multicolumn{2}{|c|}{$\begin{array}{l}46 \\
474\end{array}$} & & 1.2 & & & 466 & 4 & 7 & & 27 & & \\
\hline \multirow{2}{*}{\multicolumn{2}{|c|}{676}} & & -3 & & & 474 & & 5 & & 2 & & \\
\hline & & & 2 & & & 670 & 2 & 2 & & 2 & & \\
\hline \multirow{3}{*}{ No. } & \multirow{3}{*}{\multicolumn{3}{|c|}{ Descelption }} & \multirow{3}{*}{ Dist. } & \multicolumn{3}{|c|}{ DOSERATES } & \multicolumn{5}{|c|}{ CONTAMMUATIOS LEVELLS } \\
\hline & & & & & \multirow{2}{*}{$\begin{array}{l}\text { Shallow } \\
\text { metsesh }\end{array}$} & \multirow{2}{*}{ 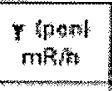 } & \multirow{2}{*}{ matersh } & Total 010 & $80 \cos ^{2}$ & \multicolumn{3}{|c|}{ Removatle $1 / 100$ mon 2} \\
\hline & & & & & & & & adpment & a ldem? & 8 idpmat & e idpmi & merding \\
\hline 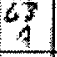 & & Bes & Auscet & $j^{\circ}$ & 482 & 18 & hith. & & & & & $\mu^{4}$ \\
\hline 4 & & & & $y^{\prime \prime}$ & 435 & $\angle 5^{5}$ & & & & & & $\angle$ \\
\hline it & & & & $Y^{\prime \prime}$ & 647 & 12 & & & & & & \\
\hline 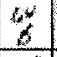 & & & & $1^{*}$ & 1177.5 & 225 & & & & & & \\
\hline 5 & & & & $r$ & $205 y$ & 42 & & & & & & \\
\hline 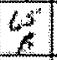 & & & & $t^{\prime \prime}$ & 2058 & 42 & & & & & & \\
\hline$\Delta y$ & & & & $t^{\text {ir }}$ & 345 & $-1,5$ & & & & & & \\
\hline 8 & & & & $f^{i t}$ & 4,2 & 1.5 & & & & & & \\
\hline$\frac{1}{4}$ & & & & $t^{\prime \prime}$ & 235.5 & 4,5 & & & & & & \\
\hline 8 & & & $i$ & $t^{*}$ & 237 & 3 & $\mu A$ & & & & & Dr \\
\hline \multicolumn{13}{|c|}{ Continuted on pager 2} \\
\hline \multicolumn{7}{|c|}{ 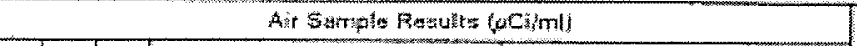 } & loggnt & (n) & & & & \\
\hline & Bz & a & In:tâl & & Decer & & $(3) \cdot 5 n$ & sar Locstio & & $A$. Air & Sampla Lo & ation \\
\hline a 1 & N4 & & & & $4 A$ & & $\operatorname{mon}$ & & & * C & & \\
\hline 8. 1 & & & & 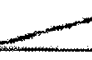 & & & {$[4]=60$} & pastas & & $*-\cos t$ & teet Ragdit & \\
\hline 2 & & & $2>$ & & & & othet,$W$ & Which & & wC: & wolla & $c h x_{2}$ \\
\hline 2 & $M H-$ & & & + & $N H^{2}$ & & & & & & & \\
\hline
\end{tabular}




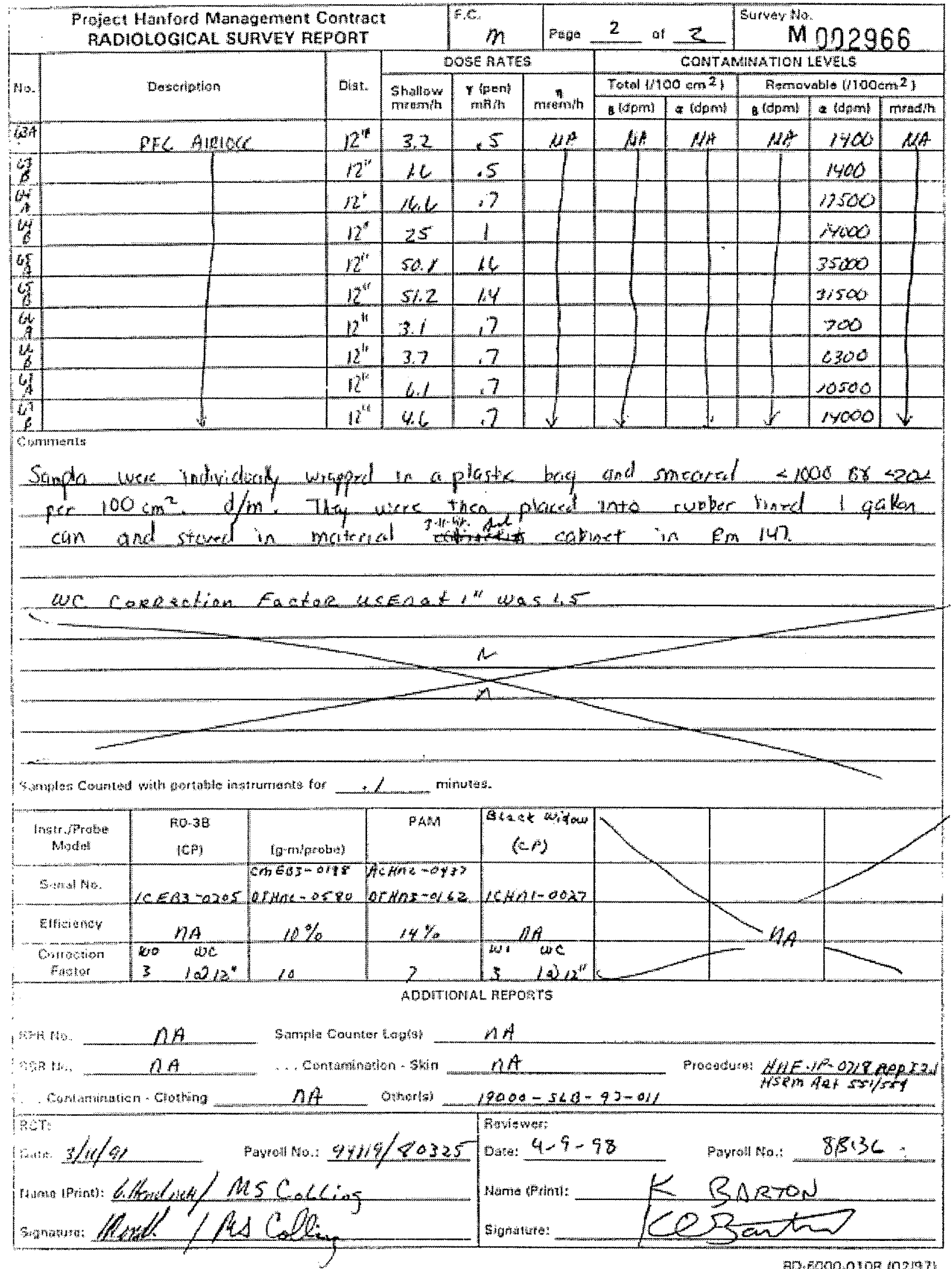

$80.6000 .064029 \%$ 


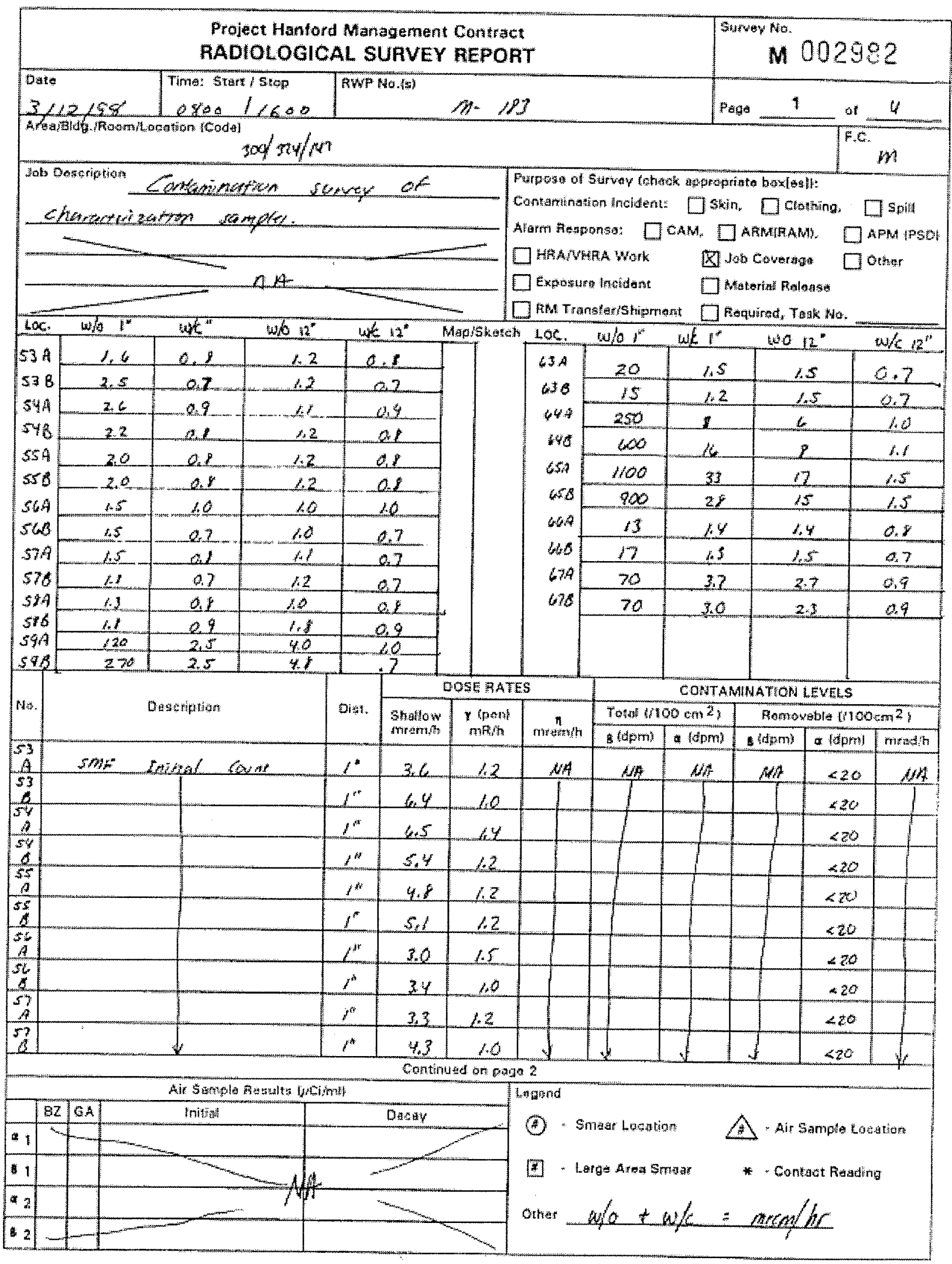

$80.6000-01010797$ 


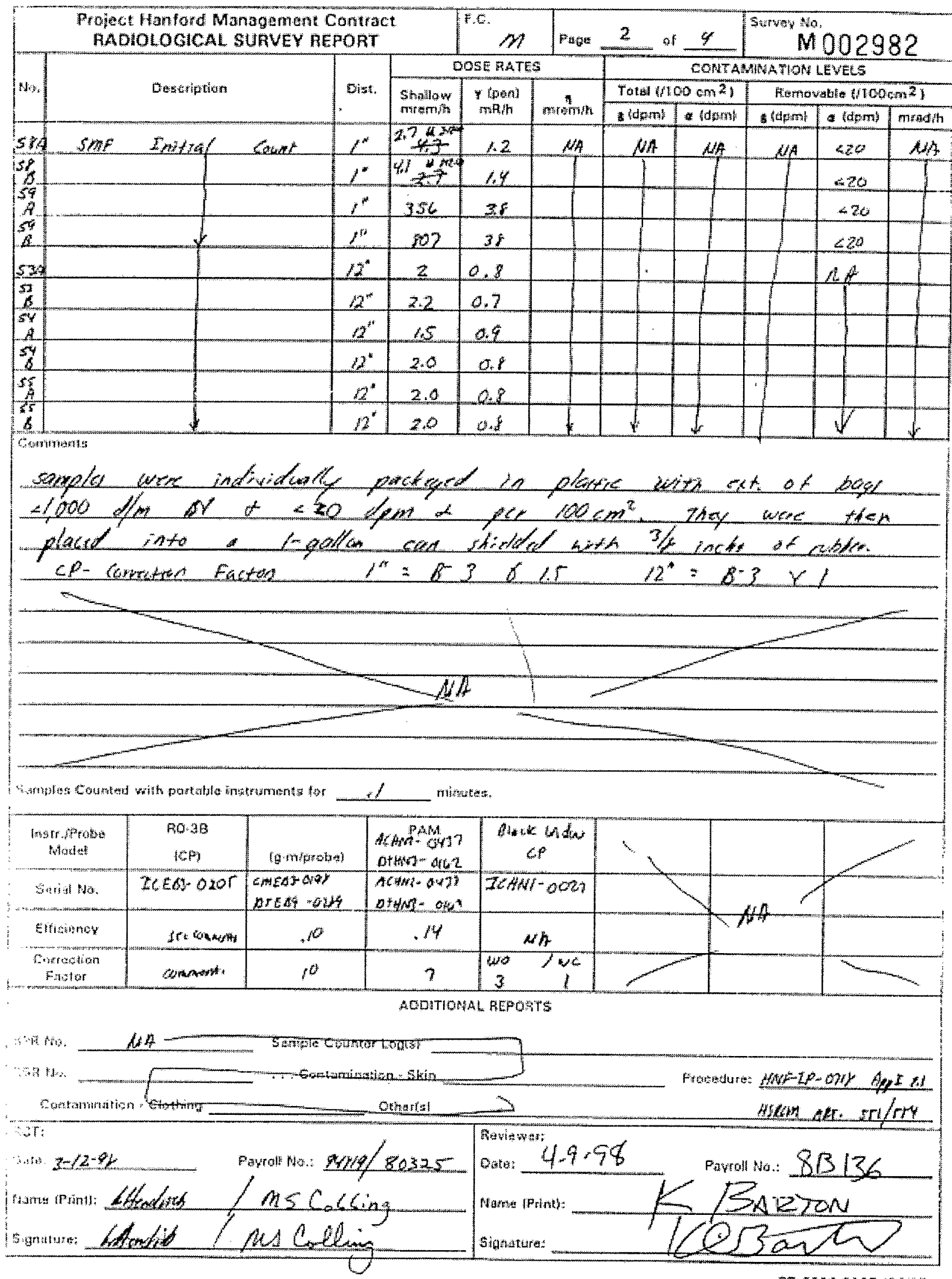




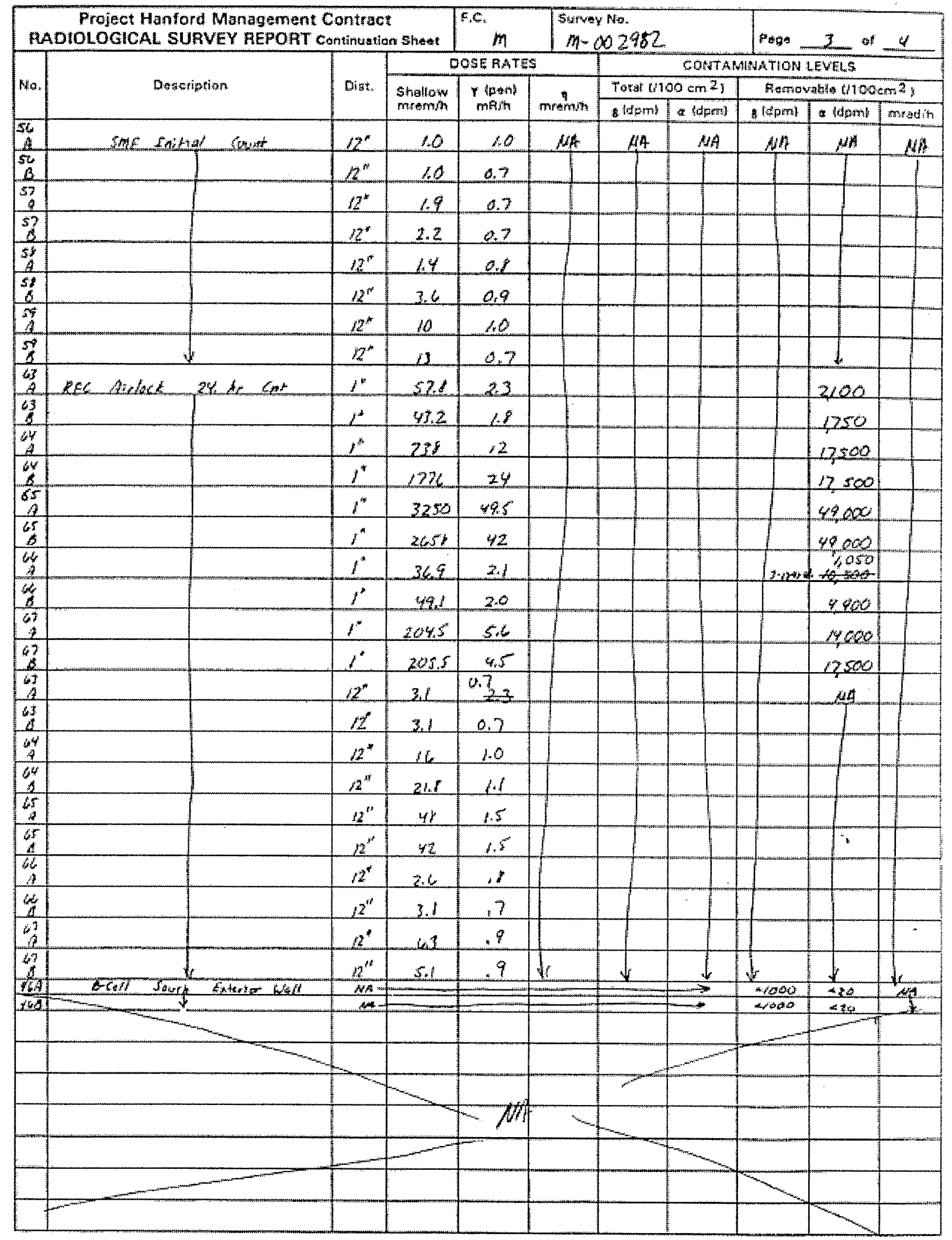

60.5000.010.1 (025) 


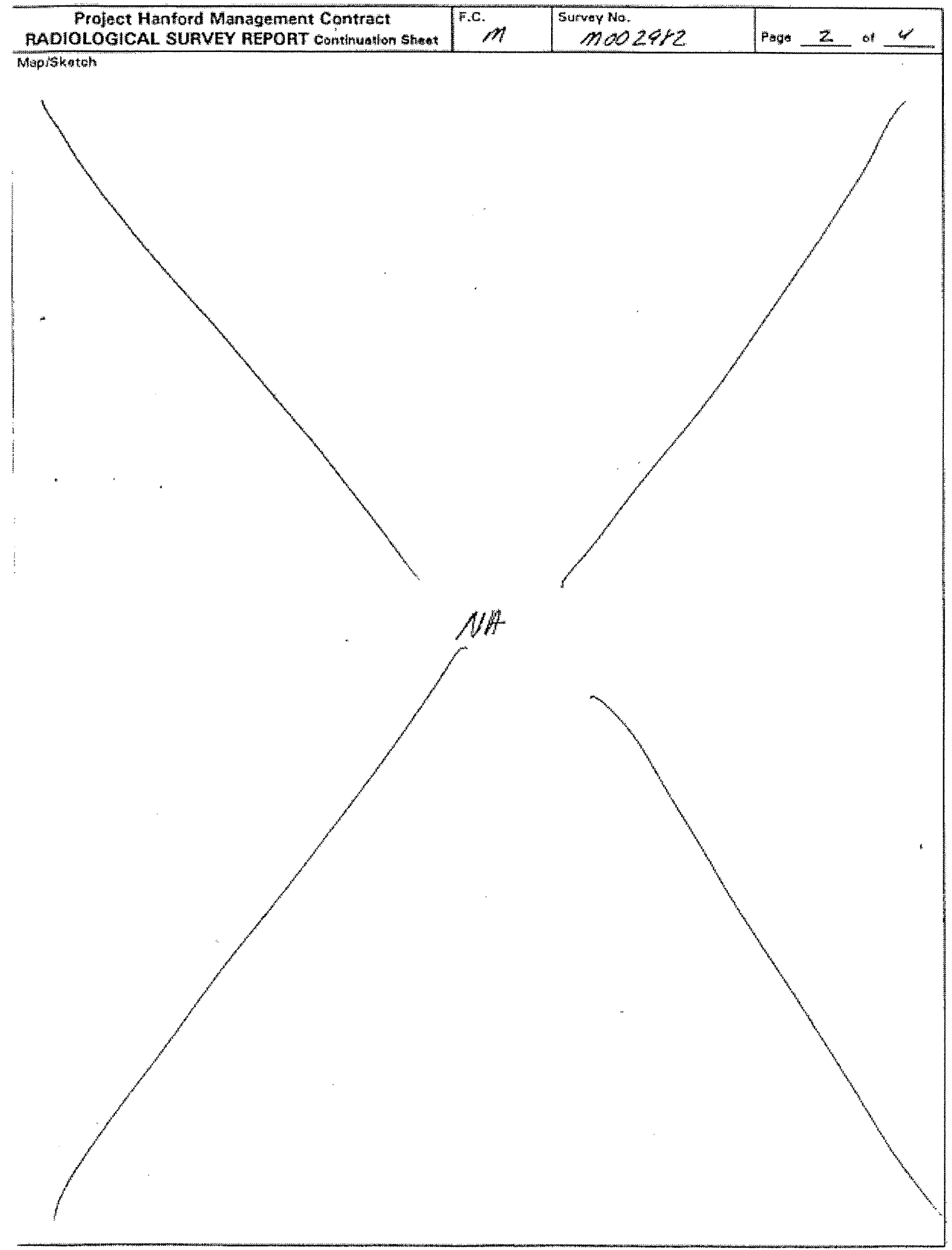

ED.50000:0.17 $10297 \%$ 


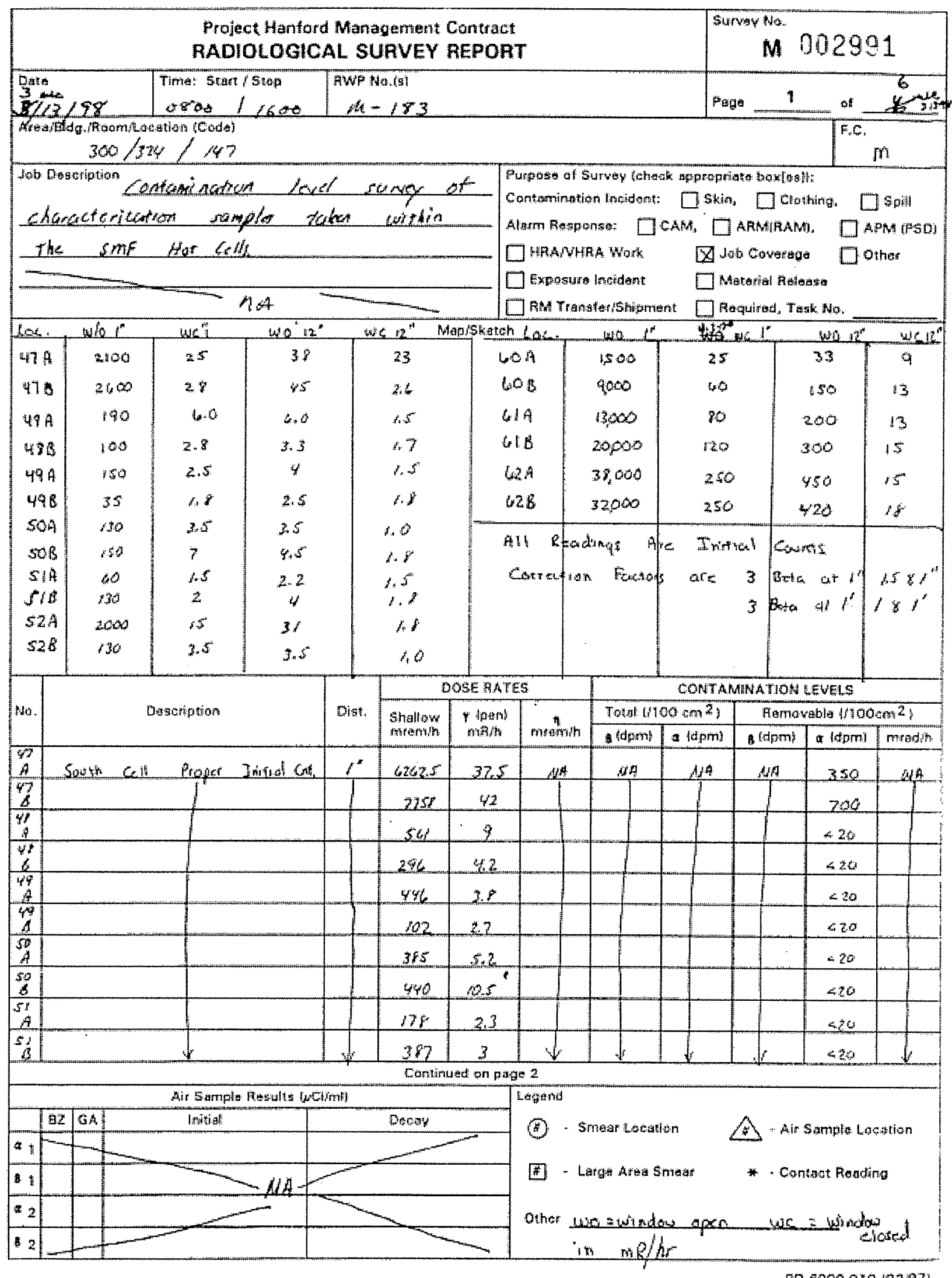




\section{APPENDIX C}

\section{LABORATORY ANALYTICAL REPORTS}


Report 98-2463, Gamma Energy Analytical Results, dated 04/23/98................................. C-1

Report 98-2463, Sr-90 Analytical Results, dated 04/24/98 ................................................. C-4

Report 98-2463, Alpha Energy Analytical Results, dated 07/28/98 .................................... C-6 
Battelle Pacific Northwest Laboratory

Radioatualytical Group - 325 Bldg.

Client: S. Landsman Wpt; K82285

Cognizant Scientist:

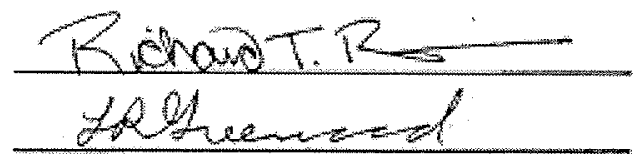

Date: $4 / 239$

Concur:

Date: $4-23-78$

Measured Activities ( $\mu$ Cissample)

\begin{tabular}{|c|c|c|c|c|c|c|c|c|}
\hline & & & & Ganma & Energy & Analys: & & \\
\hline $\begin{array}{l}\text { ALO ID } \\
\text { Client ID }\end{array}$ & $\begin{array}{l}\text { Mn-54 } \\
\text { Error\% }\end{array}$ & $\begin{array}{c}\text { Co } 60 \\
\text { Error\% }\end{array}$ & $\begin{array}{l}\text { \$b* } 125 \\
\text { Error } \%\end{array}$ & $\begin{array}{l}\mathrm{Cs} * 134 \\
\text { Error } \%\end{array}$ & $\begin{array}{l}\text { C5-137 } \\
\text { Errot \% }\end{array}$ & $\begin{array}{l}\text { Eu-154 } \\
\text { Errot\% }\end{array}$ & $\begin{array}{l}\text { Eu-155 } \\
\text { Error } \%\end{array}$ & $\begin{array}{l}\text { Am-241 } \\
\text { Error \% }\end{array}$ \\
\hline $\begin{array}{l}98 \times 2463 \\
03 a\end{array}$ & $<4, E-6$ & $<6 . E-8$ & $<4, E+5$ & $\angle 5 . E-6$ & $\begin{array}{c}4.81 \mathrm{E}-3 \\
6 \%\end{array}$ & $<2, E-5$ & $\angle 3, E-5$ & $6, E-5$ \\
\hline $\begin{array}{l}\$ 8-2464 \\
030\end{array}$ & $<5 . E \cdot 6$ & -7: & $<B, E-5$ & $<6 . E-6$ & $\begin{array}{c}1.41 \mathrm{E}-2 \\
6 \%\end{array}$ & $2 . E-5$ & $<5 . E-5$ & $<2 E 4$ \\
\hline $\begin{array}{l}98-2465 \\
04 a\end{array}$ & $<3 . E-6$ & $\angle 4, E-6$ & $\angle 3, E-5$ & $\angle 4, E-6$ & $\begin{array}{c}3.61 \mathrm{Em}^{3} 3 \\
5 \%\end{array}$ & $<9 . E S$ & $<3 . E-5$ & $<2 E-4$ \\
\hline $\begin{array}{l}98-2466 \\
04 \mathrm{~b}\end{array}$ & $\begin{array}{c}1.05 E-5 \\
32 \%\end{array}$ & $\begin{array}{c}5.97 E-4 \\
5 \%\end{array}$ & $<3 . E-5$ & $47 \equiv-6$ & $\begin{array}{c}2.40 \mathrm{E}-3 \\
5 \%\end{array}$ & $<2, E-5$ & $<3, E-5$ & $<2, E-4$ \\
\hline $\begin{array}{l}98-2467 \\
65 a\end{array}$ & $<1, E-5$ & $\begin{array}{c}9.11 E=4 \\
5 \%\end{array}$ & $4 . E-5$ & $<2=5$ & $\begin{array}{c}2.20 \mathrm{E}-3 \\
5 \%\end{array}$ & $<3 . E-5$ & $<2 . E-5$ & $<2, E-5$ \\
\hline $\begin{array}{l}98 * 2468 \\
05 b\end{array}$ & $<7, E-6$ & $\begin{array}{c}1.61 E_{-4} \\
5 \%\end{array}$ & $<8 . E-5$ & C8.E-6 & $\begin{array}{c}1.93 E-2 \\
5 \%\end{array}$ & $<3, E-5$ & $<3, E-5$ & $2 . E-5$ \\
\hline $\begin{array}{l}98-2469 \\
\text { tob }\end{array}$ & $\angle 4, E-6$ & $<4, E-6$ & $<9 . E-6$ & $\angle 4 . E \leq 5$ & $<4 E-6$ & $<8 E-6$ & $<2, E-5$ & $\begin{array}{c}6.05 E-4 \\
7 \%\end{array}$ \\
\hline $\begin{array}{l}98-2470 \\
18 a\end{array}$ & $<\theta, E-5$ & $<1 . E-4$ & $<3 . E-3$ & $\begin{array}{c}4.09 E-3 \\
5 \%\end{array}$ & $\begin{array}{c}1.10 E \div 0 \\
5 \%\end{array}$ & $\begin{array}{c}2,25 E-3 \\
5 \%\end{array}$ & $<1, E_{-3}$ & $\begin{array}{c}1.51 \mathrm{E}-3 \\
29 \%\end{array}$ \\
\hline $\begin{array}{l}98-2471 \\
18 \mathrm{~b}\end{array}$ & $<8 . E-5$ & $<2 . E-4$ & $<2$ E-3 3 & $\begin{array}{c}7,14 E-4 \\
10 \%\end{array}$ & $\begin{array}{c}2.38 E-1 \\
5 \%\end{array}$ & $\begin{array}{c}1.33 E-3 \\
8 \%\end{array}$ & $<8 . E-4$ & $\begin{array}{c}1.37 \mathrm{E}-3 \\
32 \%\end{array}$ \\
\hline $\begin{array}{l}98 \times 2472 \\
19 a\end{array}$ & $<9, E-5$ & $\begin{array}{c}2.93 E-4 \\
11 \%\end{array}$ & $<3 . E-3$ & $<2 . E-4$ & $\begin{array}{c}5.26 \mathrm{E}-1 \\
5 \%\end{array}$ & $\begin{array}{c}2.25 \mathrm{E}-3 \\
6 \%\end{array}$ & $\begin{array}{c}1.61 E-3 \\
20 \%\end{array}$ & $\begin{array}{c}2.94 E-3 \\
20 \%\end{array}$ \\
\hline $\begin{array}{l}98-2473 \\
196\end{array}$ & $<9 . E-5$ & $<2, E-4$ & $<3, E-3$ & $\begin{array}{c}8.05 E-4 \\
10 \%\end{array}$ & $\begin{array}{c}5.77 \mathrm{E}=1 \\
5 \%\end{array}$ & $\begin{array}{c}1.60 E-3 \\
8 \%\end{array}$ & $<2=-3$ & $<2, E \cdot 3$ \\
\hline $\begin{array}{l}98-2474 \\
20 a\end{array}$ & $<4 . E-5$ & $<5 . E-5$ & $<4 . E-4$ & $\begin{array}{c}2.68 E-4 \\
11 \%\end{array}$ & $\begin{array}{c}2.03 E-2 \\
5 \%\end{array}$ & $\begin{array}{c}9,19 \mathrm{E}-4 \\
5 \%\end{array}$ & $\begin{array}{c}2.60 \mathrm{E}-4 \\
26 \%\end{array}$ & $\begin{array}{c}3.53 E-4 \\
27 \%\end{array}$ \\
\hline $\begin{array}{l}98-2475 \\
20 b\end{array}$ & $<2 . E-4$ & $<9 . E-5$ & $<2 E-3$ & $\begin{array}{c}5.56 E-3 \\
5 \%\end{array}$ & $\begin{array}{c}2.11 \mathrm{E}-1 \\
5 \%\end{array}$ & $\begin{array}{c}6.98 \mathrm{Em} 3 \\
5 \% 0\end{array}$ & $\begin{array}{c}2.01 \mathrm{E}^{3} 3 \\
14^{0 \%}\end{array}$ & $\begin{array}{c}3.67 E-3 \\
13 \%\end{array}$ \\
\hline $\begin{array}{l}9 a-2476 \\
21 a\end{array}$ & $<9, E-5$ & $\begin{array}{c}4,06 E-4 \\
6 \%\end{array}$ & $<2 . E-3$ & $\begin{array}{c}2.34 \mathrm{E}-3 \\
5 \%\end{array}$ & $\begin{array}{c}7.04 E-1 \\
5 \%\end{array}$ & $\begin{array}{c}3.96 \mathrm{E}-3 \\
5 \%\end{array}$ & $<1 . E-3$ & $\begin{array}{c}1,90 E-3 \\
30 \%\end{array}$ \\
\hline $\begin{array}{l}98-2477 \\
21 b\end{array}$ & $<2 . E-4$ & $\begin{array}{c}8.49 \mathrm{E}-4 \\
6 \%\end{array}$ & $<3, E-3$ & $\begin{array}{c}3.65 E-3 \\
5 \%\end{array}$ & $\begin{array}{c}9.05 \mathrm{E}-1 \\
5 \%\end{array}$ & $\begin{array}{c}6.08 E-3 \\
5 \%\end{array}$ & $\begin{array}{c}1.96 E-3 \\
16 \%\end{array}$ & $\begin{array}{c}2.94 \mathrm{E}-3 \\
21 \%\end{array}$ \\
\hline \multicolumn{9}{|c|}{ Page 1 of 3} \\
\hline
\end{tabular}


Measured Activities (pCi/sample)

\begin{tabular}{|c|c|c|c|c|c|c|c|c|}
\hline & & & & Gamma & Energy & Andysis & & \\
\hline $\begin{array}{l}\text { ALO ID } \\
\text { Client ID }\end{array}$ & $\begin{array}{l}\text { Mn-54 } \\
\text { Error \% }\end{array}$ & $\begin{array}{l}\text { Co } 60 \\
\text { Error to }\end{array}$ & $\begin{array}{l}\text { Sb- } 125 \\
\text { Error \% }\end{array}$ & $\begin{array}{l}\text { Cs-134 } \\
\text { Error \% }\end{array}$ & $\begin{array}{l}\text { Csmi37 } \\
\text { Entor\% }\end{array}$ & $\begin{array}{l}\text { Eut-154 } \\
\text { Error } \$ \%\end{array}$ & $\begin{array}{l}\text { Evt-155 } \\
\text { Error \% }\end{array}$ & $\begin{array}{l}\text { Am-2A1 } \\
\text { Exror }\end{array}$ \\
\hline $98-2478$ & 27.55 & $4.22 \mathrm{E} \cdot 5$ & $<7.5-4$ & $2.34 \mathrm{E}_{\mathrm{m}}$ & $9.09 E-2$ & $2.77 E-3$ & $8.85 E 4$ & $4.09 E-4$ \\
\hline $22 a$ & & $45 \%$ & & $5 \%$ & $5 \%$ & $5 \%$ & $17 \%$ & $56 \%$ \\
\hline $\begin{array}{l}98-2479 \\
22 b\end{array}$ & $=0, E-5$ & $2 . E-5$ & $<7 . E<4$ & $\begin{array}{c}1,10 \mathrm{E}-3 \\
5 \%\end{array}$ & $\begin{array}{c}79+E-2 \\
5 \%\end{array}$ & $\begin{array}{l}1.98 E-3 \\
5 \%\end{array}$ & $=4 . E-4$ & $\begin{array}{c}1.09 E=3 \\
23 \%\end{array}$ \\
\hline $\begin{array}{l}9 B-2480 \\
23 a\end{array}$ & $<1.2-4$ & $\begin{array}{c}1.26 E-4 \\
28 \%\end{array}$ & $<2 . E-3$ & $49 . \pm-5$ & $\begin{array}{c}3,46 \mathrm{E}-1 \\
5 \%\end{array}$ & $<3, E-4$ & $<9 . E_{-4}$ & $<2, E-3$ \\
\hline $\begin{array}{l}98 \cdot 2481 \\
23 b\end{array}$ & $<1 . E-4$ & $<2 E-4$ & $<2 E-3$ & $2 . E 4$ & $\begin{array}{c}2.45 \mathrm{E}-1 \\
5 \%\end{array}$ & $<6, E-4$ & $\angle 8, E-4$ & $=2 E-3$ \\
\hline $\begin{array}{l}98-2482 \\
24 a\end{array}$ & $\angle 6, E-6$ & $49 . E-6$ & $<5.44$ & $<6 . E-5$ & $\begin{array}{c}5.04 E-2 \\
5 \%\end{array}$ & $<2, E-4$ & $<3 E-4$ & $8, E-4$ \\
\hline $\begin{array}{l}98-2483 \\
24 b\end{array}$ & $<3 E-5$ & $\angle 4 E-5$ & $<4 E-4$ & $\angle 4, E-5$ & $\begin{array}{c}3.12 E-2 \\
5 \%\end{array}$ & $<2 E-4$ & $2 \mathrm{E}-4$ & $\triangle 3 . E-4$ \\
\hline $\begin{array}{l}98-2484 \\
25 a\end{array}$ & $<4 E-5$ & $<E^{-5}$ & $2 . E-4$ & $<4, E-5$ & $\begin{array}{c}3.45 E-3 \\
5 \%\end{array}$ & $\angle T E 4$ & $2 . E 4$ & $<2, E-4$ \\
\hline $\begin{array}{l}98-2485 \\
25 b\end{array}$ & $44, E-5$ & $\angle B E-5$ & $<1 . E$ & $\angle 4 . E 5$ & $\begin{array}{l}9.25 E-4 \\
5 \%\end{array}$ & $\angle 8 E-5$ & $48 . E-5$ & 2. $E-4$ \\
\hline $\begin{array}{l}98-2486 \text { PB } \\
\text { Process Blank }\end{array}$ & $<4 E-4$ & 26.54 & $* 1, E-3$ & $<3=-4$ & $\begin{array}{c}3.48 E-2 \\
2 \%\end{array}$ & $<7 . E-4$ & <8.E-4 & $<2 . E-3$ \\
\hline $\begin{array}{l}99-2486 \\
26 a\end{array}$ & $<3, E-3$ & $\begin{array}{c}247 \mathrm{E}-2 \\
5 \%\end{array}$ & $\approx 5 . E=2$ & $\begin{array}{c}1.69 E-2 \\
9 \%\end{array}$ & $\begin{array}{c}1.31 E+1 \\
2 \%\end{array}$ & $\begin{array}{c}4.20 E-2 \\
6 \%\end{array}$ & C E-2 & $\begin{array}{c}7.29 \mathrm{E}-2 \\
22 \%\end{array}$ \\
\hline $\begin{array}{l}98-2487 \\
26 \mathrm{~b}\end{array}$ & $<3 . E=3$ & $\begin{array}{c}4.73 E-2 \\
4 \%\end{array}$ & $<5 . E-2$ & $\begin{array}{c}292 E-2 \\
6 \%\end{array}$ & $\begin{array}{c}1.70 E+1 \\
2 \%\end{array}$ & $\begin{array}{c}8.43 E-2 \\
5 \%\end{array}$ & $<3 . E-2$ & $\begin{array}{c}1.51 E-1 \\
14^{\circ} \%\end{array}$ \\
\hline
\end{tabular}

Note: Sample $26 \mathrm{~b}$ also has Ag-108m activity at 1.59E-2 +f-18\% LCirsample.

\begin{tabular}{|c|c|c|c|c|c|c|c|c|}
\hline $\begin{array}{l}98-2488 \\
27 a\end{array}$ & $<4, E-3$ & $\begin{array}{c}6.35 E-2 \\
3 \%\end{array}$ & $<6, E-2$ & $\begin{array}{c}2.94 E \cdot 2 \\
7 \%\end{array}$ & $\begin{array}{c}2.81 E+1 \\
2 \%\end{array}$ & $\begin{array}{c}6.34 \mathrm{E}=2 \\
6 \%\end{array}$ & $\therefore 4.2$ & $\begin{array}{c}1.13 E-1 \\
20 \%\end{array}$ \\
\hline $\begin{array}{l}98-2489 \\
27 b\end{array}$ & $<2 \mathrm{E}-3$ & $\begin{array}{c}2.81 \mathrm{E} \cdot 2 \\
4 \%\end{array}$ & $\angle 4, E-2$ & $\begin{array}{c}9.76 E-3 \\
14 \%\end{array}$ & $\begin{array}{c}1.26 E+1 \\
2 \%\end{array}$ & $\begin{array}{c}1.84 E-2 \\
10 \%\end{array}$ & $\angle 3 E \cdot 2$ & $\angle A, E-2$ \\
\hline $\begin{array}{l}98-2490 \\
28 a\end{array}$ & $\left\langle 2 E_{n 1} 1\right.$ & $\begin{array}{c}1.31 E+0 \\
5 \%\end{array}$ & $<4 . E+0$ & $<3 E-1$ & $\begin{array}{c}1.25 E \div 3 \\
3 \%\end{array}$ & $\begin{array}{c}6.99 E+0 \\
20 \%\end{array}$ & $<2 . E+0$ & $2 E \div 0$ \\
\hline $\begin{array}{l}98-2491 \\
28 b\end{array}$ & $<9 . E-3$ & $\begin{array}{c}9.41 \mathrm{E}-2 \\
5 \%\end{array}$ & $<2, E-1$ & $\begin{array}{c}4.44 E-2 \\
13 \%\end{array}$ & $\begin{array}{c}4.92 E=1 \\
2 \%\end{array}$ & $\begin{array}{c}8.24 \mathrm{E} \cdot 2 \\
10 \%\end{array}$ & $<2, E-1$ & $<2, E-1$ \\
\hline $\begin{array}{l}98-2493 \\
300\end{array}$ & $<7, E-3$ & $\begin{array}{c}1.45 E-2 \\
17 \%\end{array}$ & 2.E-1 & $\begin{array}{c}2,28 \mathrm{E}-2 \\
19 \%\end{array}$ & $\begin{array}{c}4,36 E+1 \\
2 \%\end{array}$ & $\begin{array}{c}1,46 \mathrm{E}-2 \\
31 \%\end{array}$ & $<2 . E=1$ & $=2, E-1$ \\
\hline $\begin{array}{l}98-2494 \\
30 b\end{array}$ & $<2, E * 3$ & $\begin{array}{c}7.28 \mathrm{E}-3 \\
9 \%\end{array}$ & $\angle 4, E-2$ & $\begin{array}{c}1.78 \mathrm{E}-3 \\
47 \%\end{array}$ & $\begin{array}{c}1.21 \mathrm{E} * 1 \\
2 \%\end{array}$ & $\begin{array}{c}1.19 \mathrm{E}-2 \\
14 \%\end{array}$ & <3.Ë-2 & $\begin{array}{c}4,01 E-2 \\
36 \%\end{array}$ \\
\hline
\end{tabular}


Measured Activities (pCirsample)

\begin{tabular}{|c|c|c|c|c|c|c|c|c|}
\hline & & & & Gamma & Energy & Analysis & & \\
\hline ALO ID & Mr-54 & Cosp & $8 \mathrm{~b}-125$ & $\mathrm{Cs}-134$ & $6 s-137$ & E1:-154 & Eu-155 & $\mathrm{Am}-241$ \\
\hline Client DD & error $\%$ & Errar \% & Error 8 & Errot of & Error \% & Error \% & Error \% & Error 6 \\
\hline $96-2495-P B$ & 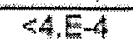 & $1.35 \mathrm{E}-1$ & $\times 9 . E 4$ & $2,17 E-1$ & $4.57 E+2$ & $1.80 \mathrm{E}-1$ & $\times 8 . E_{4}^{4}$ & $<5, E-1$ \\
\hline Process Blank & & $7 x$ & & $13 \%$ & $2 \%$ & $23 \%$ & & \\
\hline $98-2495$ & \&3.E.2 & $6.50 \mathrm{E}-2$ & $65 . E-1$ & $1.0 \mathrm{ge} \times \frac{}{\frac{1}{2}}$ & 2,3 SE 2 & $6.26 \mathrm{E}$ & $<3 . E-1$ & <7.E-1 \\
\hline $39 a$ & & $11 \%$ & & $15 \%$ & $2 \%$ & $36 \%$ & & \\
\hline $98 \times 2496$ & $\therefore 4 E 2$ & $<2 E 2$ & $<A, E-1$ & $<4 .=-2$ & $1.68 E * 2$ & $2.87 E-1$ & $<2=-1$ & 4. E- 1 \\
\hline $39 b$ & & & & & $2 \%$ & $8 \%$ & & \\
\hline $96-2497$ & $<3, E-3$ & $1.02=-2$ & $<\cdot E-2$ & $<4.53$ & $7,705+0$ & $2.07 E-2$ & $<3=2$ & $\angle 4 E-2$ \\
\hline $40 a$ & & $11 \%$ & & & $2 \%$ & $16 \%$ & & \\
\hline $98-2498$ & $-3, E-3$ & $4, E_{-3}$ & $<7, E-2$ & $\angle 4 . E-3$ & $7.16 \mathrm{E}+\mathrm{Q}$ & $1.73 E-2$ & $<4 . E 2$ & <6.E-2 \\
\hline 400 & & & & & $2 \%$ & $20 \%$ & & \\
\hline $98-2501$ & \&5.E-3 & $\angle 6: E-3$ & $<2 E-1$ & $<8, E_{3} 3$ & 4.30E+1 & $1,67 \mathrm{E}-1$ & $<8 . E-2$ & $1.665-1$ \\
\hline $42 a$ & & & & & $2 \%$ & $7 \%$ & & $25 \%$ \\
\hline $98-2502$ & $<2 E-2$ & 2. E.E-2 & $4 . E-1$ & $<2 . E=2$ & $1.28 E+2$ & $5.25 E_{-1}-1$ & $<2 . E-1$ & $6.49 E-1$ \\
\hline $42 \mathrm{~b}$ & & & & & $2 \%$ & $4 \%$ & & $14 \%$ \\
\hline $98-2503$ & $<2, E 3$ & $<4, E-3$ & $<3,8-2$ & $<2 . E \times 3$ & $1.82 E \div 0$ & $<0 . E-3$ & $<2 . E-2$ & $<3 . E-2$ \\
\hline $43 a$ & & & & & $2 \%$ & & & \\
\hline $96-2504$ & $-4 \cdot E+3$ & $<5=-3$ & $<2 E-1$ & $<0 . E-3$ & $2.2 \mathrm{gE} \div 1$ & $5.33=-2$ & $-6 . E-2$ & $<8 . E 2$ \\
\hline 430 & & & & & 28 & $9 \%$ & & \\
\hline $98-2505$ & $<3, E-3$ & $<4, E-3$ & <2.E-1 & $* 6 . E-3$ & $2.51 \mathrm{E}: 1$ & $6.59=2$ & $<6 . E-2$ & $\leq 7 . E-2$ \\
\hline $44 a$ & & & & & $2 \%$ & $7 \%$ & & \\
\hline $98-2506$ & $<2 . E-3$ & 44.5 & $<4 . E-2$ & $<3 . E-3$ & $4.90 E \div 0$ & $1.16 E-2$ & $<2, E-2$ & $<4 . \pm-2$ \\
\hline $44 b$ & & 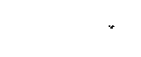 & & & $20 \%$ & $14 \%$ & & \\
\hline $90-2507$ & $<6, E-3$ & $<1 . E-2$ & $<E_{-1}$ & 7.E. & 5.34E & $8.4 E-2$ & $<g-2$ & $<2, E * 1$ \\
\hline $45 a$ & & & & & $2 \%$ & $9 \%$ & & \\
\hline $98-2508$ & $<3 . E_{-2}$ & $<1 . \mathbb{E}=2$ & $\angle 6 . E-1$ & $\alpha_{3} E-2$ & $3.29 \mathrm{E}+2$ & $1.71 E-1$ & $<3 . E-1$ & $2.779-1$ \\
\hline $45 \mathrm{~b}$ & & & & & $2 \%$ & $10 \%$ & & $37 \%$ \\
\hline $90-2539$ & $<5 . E$ & $1.81 \mathrm{E}-5$ & $<3, E-5$ & $\angle 8 . E-6$ & $1.53 E-3$ & $<2 . E-5$ & $<2 E-5$ & $48-6$ \\
\hline $32 a$ & & $11 \%$ & & & $5 \%$ & & & \\
\hline $98-2540$ & $<4.6$ & $6.40 E-6$ & $<3 . E=5$ & $1.53 E-5$ & $132 F-3$ & $-9 f-6$ & $<2=-5$ & $<4 . E-5$ \\
\hline $32 \mathrm{~b}$ & & $17 \%$ & & $20 \%$ & $5 \%$ & & & \\
\hline $\begin{array}{l}98 \cdot 2552 \\
38 b\end{array}$ & $<3 . E-6$ & $\triangle 5 . E-6$ & -3.E-6 & $\angle 4 . E$ & $-4 . \pm 56$ & 49.6 & $\alpha=-5$ & $<6 . E-5$ \\
\hline $98-2304$ & $60 \pm 2$ & $1.38 E * 1$ & $<8 . E-1$ & 2.17E-1 & $4.57 E+2$ & $1.80 E-1$ & $\alpha=$ & $x \mathrm{C}=1$ \\
\hline $31 \mathrm{a}$ & & $7 \%$ & & $13 \%$ & & $\begin{array}{c}1.005=1 \\
23 \% 6\end{array}$ & $0.5=1$ & $-9,5=-1$ \\
\hline $98-2855$ & $<3 . E-2$ & $6.50 E-2$ & $<6 . E-1$ & $1,09 \mathrm{E}-1$ & $2.38=+2$ & 6.2EE-2 & $44, E-1$ & $67 . E-1$ \\
\hline $31 b$ & & $11 \%$ & & $15 \%$ & $2 \%$ & $36 \%$ & & \\
\hline
\end{tabular}

Page 3 or 
Batele Pacilic Northwast Laboratory

$98 \times 2463$

Analyhoal Chemistry Laboratory

$4 / 2498$

Radioanalytical Group - 325 gidg.

Clent: S. Laridsman

Wp\#: K82285

Cognizan Scientist: Concur:

$=\frac{M+2-42 L}{1}$

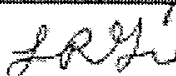

\section{ter}

Measured Activities (pCîlsample)

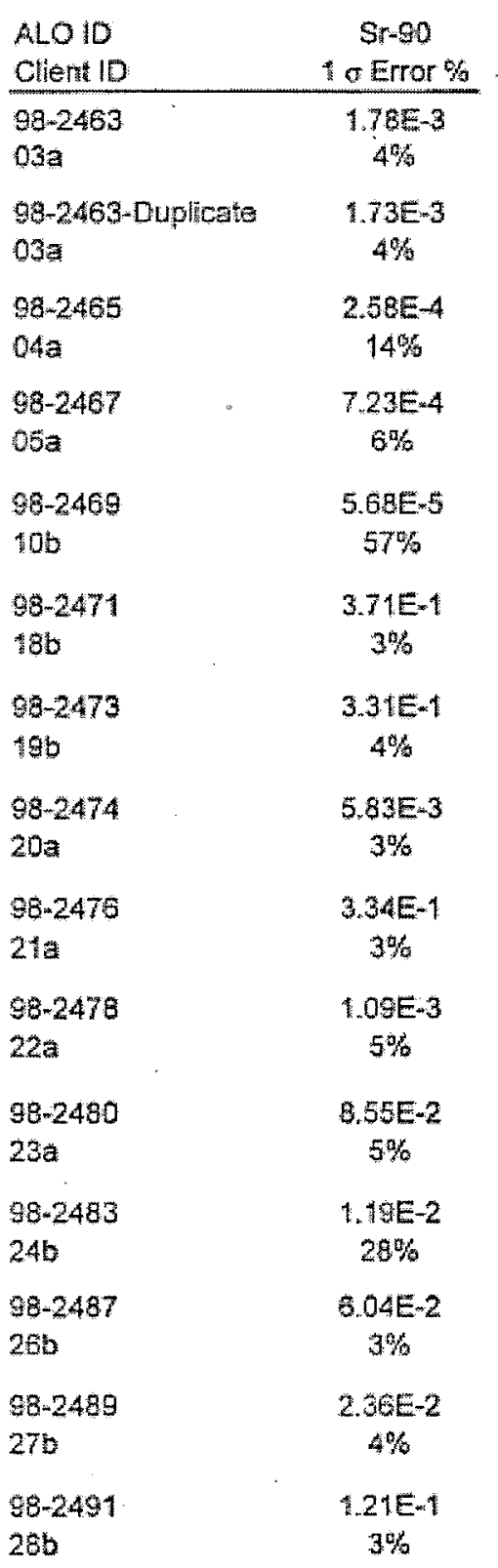

Page 1 of 2 


\begin{tabular}{|c|c|}
\hline & Measured Activities ( $\mu$ Ci/sample) \\
\hline ALO ID & $\$ r-90$ \\
\hline Client id & 10 Error $\%$ \\
\hline $98 \cdot 2493$ & 1,30E-1 \\
\hline $30 a$ & $3 \%$ \\
\hline $98.2495-\mathrm{PB}^{*}$ & $4.59 \mathrm{E}-2$ \\
\hline Process Blank & $18 \%$ \\
\hline $98 \times 2495^{*}$ & $7,32 E+1$ \\
\hline $39 \mathrm{~b}$ & $3 \%$ \\
\hline $98-2498^{*}$ & $3.84 E+0$ \\
\hline $40 \mathrm{~b}$ & $4 \%$ \\
\hline $98-2501 *$ & $4,30 \mathrm{E}+1$ \\
\hline $42 a$ & $4 \%$ \\
\hline $98-2503^{*}$ & $2.57 E+0$ \\
\hline $43 a$ & $5 \%$ \\
\hline $98-2505^{*}$ & $1.89 \mathrm{E}+0$ \\
\hline $44 b$ & $6 \%$ \\
\hline $98-2507^{*}$ & $2.89 E+1$ \\
\hline $46 a$ & $5 \%$ \\
\hline \multirow{2}{*}{\multicolumn{2}{|c|}{$\begin{array}{l}98-2856 \\
31 b\end{array}$}} \\
\hline & \\
\hline \multirow[t]{2}{*}{ Standards } & $96 \%$ \\
\hline & $92 \%$ \\
\hline \multirow[t]{2}{*}{ Sample Splkes } & $93 \%$ \\
\hline & $94 \%$ \\
\hline \multirow[t]{2}{*}{ Blanks } & $<9 . E-5$ \\
\hline & $<a, E-6$ \\
\hline
\end{tabular}

Page 2 of 2 


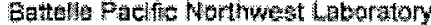

Analyticat Chemiatry Laboraion

Radivanalytical Group * 323 Bidg.

Cliezt: 5. Landsman

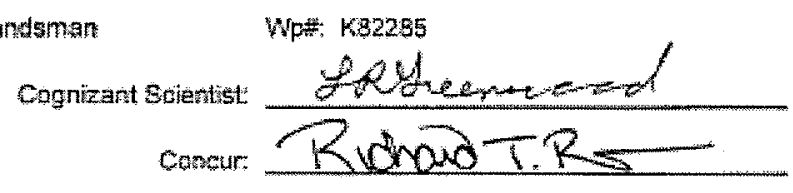

9.

7 rorge

ok to release
Date: $2 / 28 / 98$ tary 7 luga

Date: $2 / 28 / 98$

Mensured Activities fuCilsample

\begin{tabular}{|c|c|c|c|c|c|}
\hline $\begin{array}{l}\text { Alo 10 } \\
\text { clent Io }\end{array}$ & $\begin{array}{r}\text { Total Apha } \\
\text { Error }\end{array}$ & $\begin{array}{l}\text { Pu-239/2,40 } \\
\text { Enror }\end{array}$ & $\begin{array}{c}\text { Pu-23a/Am-241 } \\
\text { Errot } \%\end{array}$ & $\begin{array}{c}\text { Corv243/244 } \\
\text { Error } 8\end{array}$ & $\begin{array}{l}\text { Cor. } 242 \\
\text { Error \% }\end{array}$ \\
\hline $\begin{array}{l}9 e-2463 \\
\text { osta }\end{array}$ & $2 E-5$ & $\alpha 1 . E$ & $41, E \times 5$ & <t. E-5 & 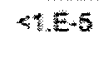 \\
\hline $\begin{array}{l}98-2464 \\
03 b\end{array}$ & $2, E, 5$ & $+E-5$ & $=1, E+5$ & 琒.E-5 & $<$ E E-5 \\
\hline $\begin{array}{l}962465 \\
04 a\end{array}$ & $<2 . E-5$ & 43.5 & $<3 . E-8$ & $B . E-6$ & $=3, E-6$ \\
\hline $\begin{array}{l}98-2466 \\
046\end{array}$ & $=2.5$ & 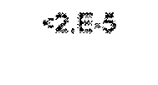 & $2 x^{2}=5$ & $2 . E=5$ & $<2 . E-5$ \\
\hline $\begin{array}{l}98-2467 \\
659\end{array}$ & 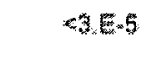 & 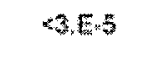 & 53 & $<3 . E-5$ & $<3 . E-5$ \\
\hline $\begin{array}{l}98-2460 \\
056\end{array}$ & $\begin{array}{c}\text { 1.4GE-5 } \\
34 \%\end{array}$ & $<8 . E-6$ & $\begin{array}{c}9.31 \mathrm{E}-\mathrm{s} \\
42 \mathrm{k}\end{array}$ & $\approx \mathrm{E}-\mathrm{E}$ & 2.6 \\
\hline $\begin{array}{l}93-2439 \\
10 b\end{array}$ & $\begin{array}{c}5.92 E=5 \\
15 \% 3\end{array}$ & $\begin{array}{c}344 \mathrm{E}-5 \\
13 \%\end{array}$ & $\begin{array}{c}2.40 \mathrm{E}-5 \\
20 \%\end{array}$ & $<3, E$ & $2 E-6$ \\
\hline $\begin{array}{l}98-2470 \\
1.8 a\end{array}$ & $\begin{array}{c}1.53 \mathrm{E}-2 \\
3 \%\end{array}$ & $\begin{array}{c}5.69 E^{-4} \\
21 \%\end{array}$ & $\begin{array}{c}3.27 E-3 \\
110\end{array}$ & $\begin{array}{c}1.15 E-2 \\
6 \%\end{array}$ & $-3 \mathrm{E}$ \\
\hline $\begin{array}{l}98-2470 \text { Dus } \\
\text { Ba }\end{array}$ & $\begin{array}{c}1.50 \mathrm{E}-2 \\
3 \%\end{array}$ & $\begin{array}{c}6.33 E-4 \\
21 \% 3\end{array}$ & $\begin{array}{c}2.22 E-3 \\
11 \%\end{array}$ & $\begin{array}{c}1.15 E-2 \\
6 \%\end{array}$ & $=3 . E-5$ \\
\hline $\begin{array}{l}98-2,71 \\
18 b\end{array}$ & $\begin{array}{c}7.55 E-3 \\
3 \%\end{array}$ & $\begin{array}{c}7.45-4 \\
26 \%\end{array}$ & $\begin{array}{c}1.79 E-3 \\
15 \%\end{array}$ & $\begin{array}{c}5.0 S E-3 \\
13\end{array}$ & 41.5 \\
\hline $\begin{array}{l}98-2472 \\
19 a\end{array}$ & $\begin{array}{c}5.62 E \cdot 3 \\
4 \%\end{array}$ & & & & \\
\hline $\begin{array}{l}98 * 2473 \\
190\end{array}$ & $\begin{array}{c}1.5 \% E-2 \\
3 \%\end{array}$ & $<1 . E-3$ & $\begin{array}{c}4.465 \% \\
26 \%\end{array}$ & $\begin{array}{c}1.15 E \times 2 \\
11 \%\end{array}$ & $41 . E-4$ \\
\hline $\begin{array}{l}90 \cdot 3474 \\
200\end{array}$ & $\begin{array}{c}1.73 \mathrm{Em} \\
5 \%\end{array}$ & $\approx 1, E-4$ & $\begin{array}{c}2035-4 \\
26 \%\end{array}$ & $\begin{array}{c}1.53 E=3 \\
10 \%\end{array}$ & $\times 1, E \times 5$ \\
\hline $\begin{array}{l}9 \mathrm{~B}=2 \mathrm{k} 75 \\
2 \mathrm{De}\end{array}$ & $\begin{array}{c}6.53 E-2 \\
3 \%\end{array}$ & & & & \\
\hline $\begin{array}{l}98-2+76 \\
213\end{array}$ & $\begin{array}{c}3.82 \mathrm{E}-2 \\
30.6\end{array}$ & स. $E-3$ & $\begin{array}{c}920 E-3 \\
21 \%\end{array}$ & $\begin{array}{c}2.90 E-2 \\
9 \%\end{array}$ & $<1 . E-4$ \\
\hline $\begin{array}{l}98-247 ? \\
216\end{array}$ & $\begin{array}{c}7.20 E-2 \\
3 \%\end{array}$ & & . & & . \\
\hline $\begin{array}{l}98-2478 \\
22 a\end{array}$ & $\begin{array}{c}4.43 E-4 \\
7 x\end{array}$ & $4 . \pm-5$ & $\begin{array}{c}464 E .5 \\
26 \%\end{array}$ & $\begin{array}{c}3.97 E-4 \\
13 \%\end{array}$ & $<1.8-5$ \\
\hline
\end{tabular}

Page 1 of 3 
Measured Actulties (uClisample)

\begin{tabular}{|c|c|c|c|c|c|}
\hline $\begin{array}{l}\text { ALO 10 } \\
\text { Chemt iD }\end{array}$ & $\begin{array}{l}\text { Total Alpha } \\
\text { Error }\end{array}$ & $\begin{array}{c}\text { Pu-z39240 } \\
\text { Error\% }\end{array}$ & $\begin{array}{c}\text { Pu-2sidam-741 } \\
\text { Entror }\end{array}$ & $\begin{array}{c}\mathrm{Cm} \cdot 243244 \\
\text { Erres }\end{array}$ & $\begin{array}{l}\mathrm{Cm}-242 \\
\text { Error }\end{array}$ \\
\hline$\frac{9 \mathrm{~B}-2479}{228}$ & $\begin{array}{c}1.63 E-2 \\
38\end{array}$ & . & & & \\
\hline $\begin{array}{l}98-2480 \\
23 a\end{array}$ & $\underset{5 \%}{2.58 E-4}$ & G.E-5 & $\begin{array}{c}1.71+4=4 \\
11 \%\end{array}$ & $\begin{array}{c}6.72 \mathrm{E}-5 \\
12 \%\end{array}$ & $<. E-6$ \\
\hline $\begin{array}{l}98-2481 \\
236\end{array}$ & $\begin{array}{c}1.48 E-3 \\
3 \%\end{array}$ & & & & \\
\hline $\begin{array}{l}96.2482 \\
249\end{array}$ & $\frac{1.01 \mathrm{e}-4}{15 \%}$ & & & & \\
\hline $\begin{array}{l}920483 \\
24 b\end{array}$ & $\begin{array}{c}2.70 E-5 \\
27 \%\end{array}$ & <2E-6̈ & $\begin{array}{l}207 \mathrm{E} \\
10 \%\end{array}$ & $\begin{array}{c}722 \mathrm{E} .6 \\
24 \%\end{array}$ & $\sigma E=7$ \\
\hline $\begin{array}{l}98.2484 \\
25 a\end{array}$ & $\begin{array}{c}7.606-6 \\
56 \%\end{array}$ & & r & & \\
\hline $\begin{array}{l}5 e_{-2455} \\
250\end{array}$ & $<2 . E-5$ & & & & \\
\hline $\begin{array}{l}\text { g8-24Be-PB } \\
\text { Process Blank }\end{array}$ & $\begin{array}{c}\text { 8.26E- } \\
9 \%\end{array}$ & $\begin{array}{c}3.40 E-4 \\
10 \%\end{array}$ & $\begin{array}{c}4.35 E-4 \\
10 \%\end{array}$ & $\begin{array}{c}5.32 \mathrm{E}-5 \\
15 \%\end{array}$ & 27.7 \\
\hline $\begin{array}{l}582466 \\
26 a\end{array}$ & $\begin{array}{c}2.67 \mathrm{E}-1 \\
3 \%\end{array}$ & & & & \\
\hline $\begin{array}{l}5 \% 2487 \\
260\end{array}$ & $\begin{array}{c}\text { 5. } \\
40 E-1\end{array}$ & $\begin{array}{c}4.02 E-2 \\
4 \%\end{array}$ & $\begin{array}{c}2.09 E-1 \\
4 \%\end{array}$ & $\begin{array}{c}2.65 E_{-1} \\
4 \%\end{array}$ & $\begin{array}{c}8.49 E-4 \\
11 \%\end{array}$ \\
\hline $\begin{array}{l}98-2488 \\
27 a\end{array}$ & $\begin{array}{c}3.20 E-1 \\
4 \%\end{array}$ & & & & \\
\hline $\begin{array}{l}58 \cdot 24 a 9 \\
27 b\end{array}$ & $\begin{array}{c}\text { 1.09E-1 } \\
5 \%\end{array}$ & $\begin{array}{c}\text { 9.EOE } \\
6 \% \\
6 \%\end{array}$ & $\begin{array}{c}4.58 \mathrm{E} / 2 \\
5 \%\end{array}$ & $\begin{array}{c}5.24 \mathrm{E}-2 \\
5 \%\end{array}$ & $\frac{1.36 E-4}{27 \%}$ \\
\hline $\begin{array}{l}96 \cdot 2490 \\
20 \pi\end{array}$ & $\begin{array}{c}2.28 E 0 \\
7 \%\end{array}$ & & & & \\
\hline $\begin{array}{l}96 \cdot 249 ! \\
28 b\end{array}$ & $\begin{array}{c}404 E-1 \\
4 \%\end{array}$ & $\begin{array}{c}2 \times 44 E-2 \\
4 \%\end{array}$ & $\begin{array}{c}1.37 \mathrm{E}-1 \\
45\end{array}$ & $\begin{array}{c}2395 \\
4 \%\end{array}$ & $\begin{array}{c}4.67 w^{4} \\
165\end{array}$ \\
\hline $\begin{array}{l}98-2493 \\
300\end{array}$ & $\begin{array}{c}8,28 E-2 \\
0 \%\end{array}$ & $\begin{array}{c}5.32 E-3 \\
7 \%\end{array}$ & $\begin{array}{c}3.305=2 \\
5 \%\end{array}$ & $\begin{array}{c}4,27 E_{-2} \\
6 \%\end{array}$ & $\begin{array}{l}2.1654 \\
22 \%\end{array}$ \\
\hline $\begin{array}{l}98 \times 2494 \\
306\end{array}$ & $\begin{array}{c}7.81 E-2 \\
6 \%\end{array}$ & & & & \\
\hline $\begin{array}{l}\text { 98-2495-PB } \\
\text { Proess alank }\end{array}$ & $\begin{array}{c}1.20 E-3 \\
12 \%\end{array}$ & $\begin{array}{c}4 \mathrm{OE}-4 \\
18 \%\end{array}$ & $\begin{array}{c}7.00 E-4 \\
16 \%\end{array}$ & $\begin{array}{c}\text { B.0DE-5 } \\
39 \%\end{array}$ & स.E-6 \\
\hline $\begin{array}{l}382495 \\
39 a\end{array}$ & $\begin{array}{c}4.26 E-1 \\
3 \%\end{array}$ & & & & \\
\hline $\begin{array}{l}98.2496 \\
300\end{array}$ & 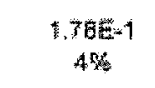 & $\begin{array}{c}1.79 E-2 \\
11 \%\end{array}$ & $\begin{array}{c}107 \mathrm{E}-1 \\
7 \%\end{array}$ & $\begin{array}{c}5.34 \mathrm{E}-2 \\
7 \%\end{array}$ & $<9 . E-5$ \\
\hline $\begin{array}{l}98-2497 \\
40 a\end{array}$ & $\frac{1,15 E-2}{5 x}$ & & & & \\
\hline $\begin{array}{l}88-2498 \\
40 b\end{array}$ & $\begin{array}{c}1.20 E-2 \\
5 \%\end{array}$ & $\begin{array}{c}1.80 E-3 \\
26 \%\end{array}$ & $\begin{array}{c}7.50 E-3 \\
9 \%\end{array}$ & $\begin{array}{c}269 \mathrm{E} \times 3 \\
13 \%\end{array}$ & $2 E-4$ \\
\hline $\begin{array}{l}98-250 \mathrm{~A} \\
42 \mathrm{a}\end{array}$ & $\begin{array}{c}3.36 E-1 \\
3 \%\end{array}$ & <2.E-Z & $\begin{array}{c}254 E-1 \\
5 \%\end{array}$ & $\begin{array}{c}\text { B. } 23 E-2 \\
5 \%\end{array}$ & $<1.2-3$ \\
\hline & & & Page 2 of 3 & & \\
\hline
\end{tabular}




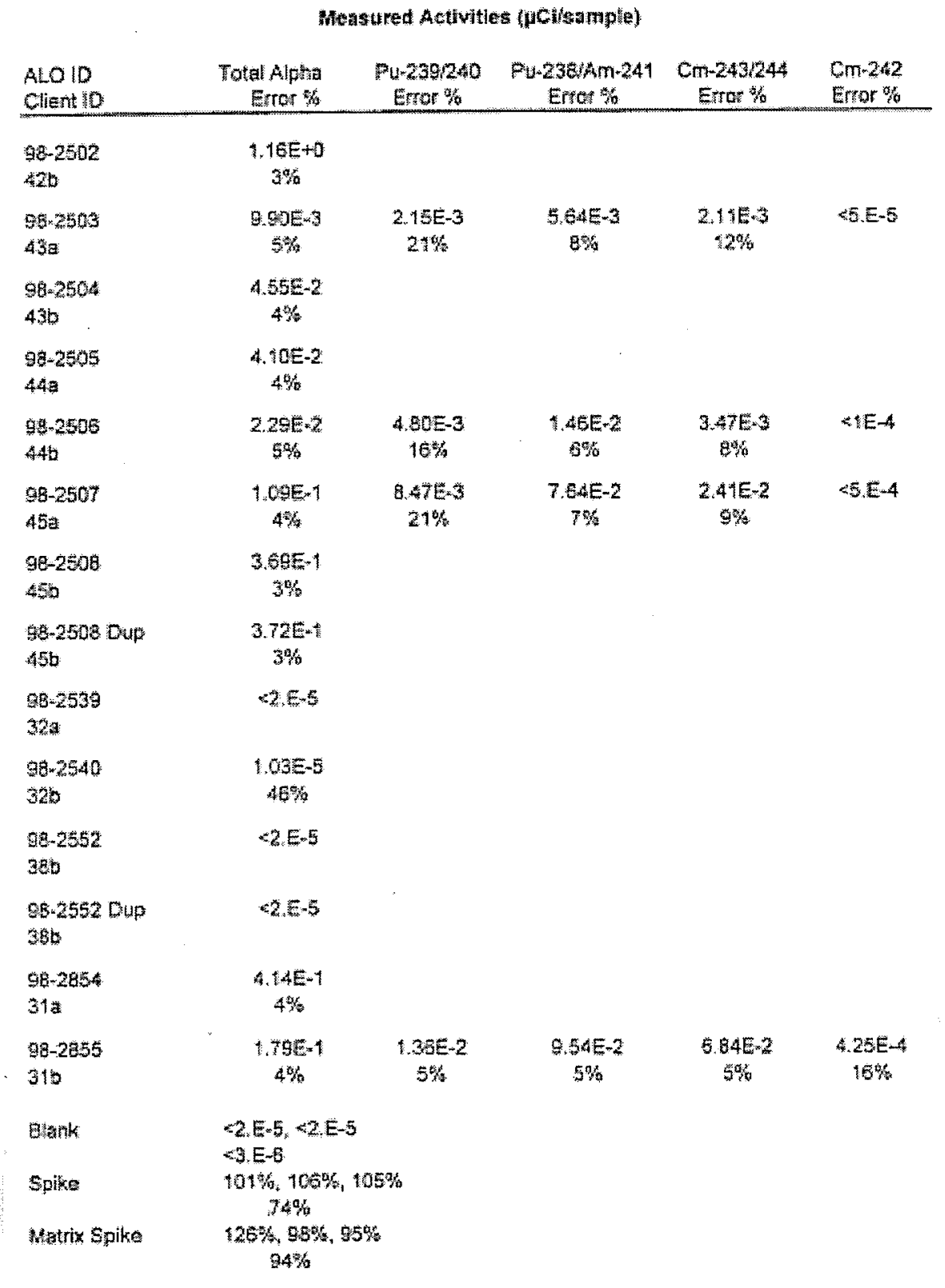

pace 3 af 3 


\section{APPENDIX D}

\section{TOTAL TO REMOVABLE ACTIVITY RATIO CALCULATION}


Total Contamination: Metal Sample Analytical Results (Source: Hill and Hobart 2000, Table 7)

\begin{tabular}{|c|c|c|c|c|c|c|c|c|c|c|c|}
\hline \multirow[b]{2}{*}{ Sample } & \multicolumn{11}{|c|}{$(\mathrm{nCi} / \mathrm{g})$} \\
\hline & ${ }^{60} \mathrm{Co}$ & ${ }^{90} \mathrm{Sr}$ & ${ }^{125} \mathrm{Sb}$ & ${ }^{137} \mathrm{Cs}$ & ${ }^{154} \mathrm{Eu}$ & ${ }^{155} \mathrm{Eu}$ & ${ }^{238} \mathrm{Pu}$ & $\begin{array}{l}{ }^{239} \mathrm{Pu} / \\
{ }^{240} \mathrm{Pu}\end{array}$ & ${ }^{241} \mathrm{Am}$ & ${ }^{243} \mathrm{Cm}$ & ${ }^{244} \mathrm{Cm}$ \\
\hline $1-1 a$ & $<48.4$ & 29,432 & $\cdots$ & 42,847 & 186.7 & $<222.4$ & 6.33 & 3.41 & 253.20 & 1.558 & 107.517 \\
\hline $1-1 b$ & $<25.6$ & 10,960 & $\cdots$ & 12,533 & 83.08 & $<93.5$ & 1.90 & 1.05 & 71.72 & 0.330 & 22.786 \\
\hline $1-2 a$ & $<42.4$ & 25,149 & -- & 30,756 & 127.7 & $<174.8$ & 5.18 & 2.99 & 222.22 & 1.366 & 94.279 \\
\hline $1-2 b$ & $<31.8$ & 14,798 & $-\cdots$ & 23,714 & 114.9 & $<127.8$ & 2.98 & 1.45 & 101.20 & 0.374 & 25.808 \\
\hline $3-1 a$ & $<35.6$ & 27,210 & $-\cdots$ & 17,773 & 123.7 & $<135.6$ & 3.76 & 2.46 & 54.46 & 0.280 & 19.340 \\
\hline $3-1 b$ & $<18.5$ & 8,000 & $-\cdots$ & 15,901 & 62.00 & $<59.8$ & 2.28 & 1.43 & 30.37 & 0.138 & 9.556 \\
\hline $3-2 a$ & $<17.5$ & 25,290 & -- & 16,497 & 61.41 & $<81$ & 3.02 & 1.99 & 57.71 & 0.285 & 19.663 \\
\hline $3-2 b$ & $<0.59$ & 15,290 & $-\cdots$ & 10,704 & 19.31 & $<9.4$ & 2.83 & 1.83 & 33.37 & 0.169 & 11.681 \\
\hline $1 \mathrm{~A}-1 \mathrm{a}$ & $<0.52$ & 5,450 & $\cdots$ & 3,116 & 4.44 & $<5.2$ & 0.47 & 0.22 & 5.72 & 0.026 & 1.784 \\
\hline 1A-1b & $<0.66$ & 9,480 & -- & 3,462 & 9.35 & $<6.4$ & 0.48 & 0.27 & 10.30 & 0.048 & 3.292 \\
\hline 1A-1C & $<0.15$ & 1,589 & $\cdots$ & 1,927 & 7.46 & $<3.9$ & 0.33 & 0.16 & 10.99 & 0.049 & 3.390 \\
\hline 1A-1d & $<0.14$ & 10,500 & $-\cdots$ & 6,669 & 9.86 & $<3.7$ & 0.69 & 0.74 & 15.80 & 0.077 & 5.323 \\
\hline $1 \mathrm{~A}-1 \mathrm{e}$ & $<0.31$ & 4,494 & --- & 1,766 & 2.98 & $<2.5$ & 0.37 & 0.17 & 3.51 & 0.015 & 1.008 \\
\hline 1A-1f & $<0.36$ & 7,615 & -- & 4,967 & 3.36 & $<5.3$ & 0.75 & 0.60 & 3.90 & 0.016 & 1.115 \\
\hline $1 \mathrm{~A}-1 \mathrm{~g}$ & $<0.28$ & 15,082 & $\cdots$ & 14,873 & 7.38 & $<8.9$ & 2.16 & 2.08 & 11.27 & 0.043 & 2.988 \\
\hline $1 \mathrm{~A}-1 \mathrm{~h}$ & $<0.38$ & 1,364 & --- & 1,847 & 1.41 & $<2.8$ & 0.14 & 0.09 & 0.99 & 0.005 & 0.341 \\
\hline $1 A-2$ & $<1.03$ & 1,959 & 81.1 & 2,377 & 3.29 & $<3.3$ & 0.50 & 0.24 & 1.98 & 0.011 & 0.735 \\
\hline $1 \mathrm{~A}-3$ & $<0.05$ & 233 & $\cdots$ & 918 & 0.19 & $<0.8$ & 0.09 & 0.04 & 0.37 & 0.002 & 0.129 \\
\hline $1 \mathrm{~A}-5$ & $<0.13$ & 749 & $<0.29$ & 500 & 1.55 & $<0.9$ & 0.45 & 0.22 & 2.27 & 0.012 & 0.796 \\
\hline 1A-7 & $<0.49$ & 1,616 & $<34.8$ & 3,089 & 9.63 & $<2.5$ & 2.25 & 0.97 & 22.74 & 0.117 & 8.090 \\
\hline $1 \mathrm{~A}-8$ & $<0.58$ & 2,226 & 2.01 & 1,937 & 4.06 & $<2.7$ & 0.39 & 0.15 & 2.75 & 0.011 & 0.748 \\
\hline $1 \mathrm{~A}-9$ & $<0.4$ & 1,640 & $-\cdots$ & 1,595 & 1.30 & $<3$ & 0.62 & 0.30 & 2.96 & 0.015 & 1.005 \\
\hline $1 \mathrm{~A}-10$ & $<0.32$ & 518 & $\cdots$ & 1,510 & 1.36 & $<2.9$ & 0.42 & 0.19 & 1.71 & 0.008 & 0.573 \\
\hline 1A-11 & $<0.54$ & 339 & $\cdots$ & 646 & 1.66 & $<2.7$ & 0.08 & 0.04 & 0.59 & 0.003 & 0.223 \\
\hline 1A-12 & $<0.12$ & 4,220 & $-\cdots$ & 4,051 & 3.27 & $<2.8$ & 0.71 & 0.69 & 5.18 & 0.023 & 1.567 \\
\hline 1A-14 & $<2.3$ & 1,660 & $\cdots$ & 904 & 7.31 & $<4.5$ & 0.93 & 0.53 & 4.65 & 0.030 & 2.070 \\
\hline 2B-1a & $<0.35$ & 184 & $\cdots$ & 424 & 1.16 & $<0.8$ & 1.51 & 1.13 & 1.77 & 0.003 & 0.203 \\
\hline $2 B-1 b$ & $<0.02$ & 139 & --- & 390 & 0.25 & $<0.3$ & 0.66 & 0.49 & 1.00 & 0.002 & 0.155 \\
\hline Average & $\mathrm{ND}$ & 8,114 & 41.56 & 8,132 & 30.72 & ND & 1.51 & 0.93 & 33.38 & 0.179 & 12.363 \\
\hline Max & $-\cdots$ & 29,432 & 81.10 & 42,847 & 186.70 & --- & 6.33 & 3.41 & 253.20 & 1.558 & 107.517 \\
\hline
\end{tabular}

Removable Contamination: Smear Analytical Results (Source: Hill and Hobart 2000, Table 9)

\begin{tabular}{|c|c|c|c|c|c|c|c|c|c|c|c|}
\hline \multirow[b]{2}{*}{ Sample } & \multicolumn{11}{|c|}{ (nCi/smear or $\left.\mathrm{nCi} / 100 \mathrm{~cm}^{2}\right)$} \\
\hline & ${ }^{60} \mathrm{Co}$ & ${ }^{90} \mathrm{Sr}$ & ${ }^{125} \mathrm{Sb}$ & ${ }^{137} \mathrm{Cs}$ & ${ }^{154} \mathrm{Eu}$ & ${ }^{155} \mathrm{Eu}$ & ${ }^{238} \mathrm{Pu}$ & $\begin{array}{l}{ }^{239} \mathrm{Pu} / \\
{ }^{240} \mathrm{Pu}\end{array}$ & $\begin{array}{l}{ }^{238} \mathrm{Pu} / \\
{ }^{241} \mathrm{Am}\end{array}$ & ${ }^{243} \mathrm{Cm}$ & ${ }^{244} \mathrm{Cm}$ \\
\hline $39 b$ & $<18.5$ & 72,428 & $-\cdots$ & 167,582 & 284 & $<195.6$ & $\cdots$ & 17.3 & 106 & 0.761 & 52.5 \\
\hline $40 b$ & $<3.5$ & 3,068 & $\cdots$ & 7,042 & 14.6 & $<35.6$ & -- & 1.16 & 6.63 & 0.037 & 2.54 \\
\hline $42 a$ & $<4.5$ & 42,228 & $-\cdots$ & 42,862 & 164 & $<85.6$ & $\cdots$ & 19.4 & 253 & 1.174 & 81.0 \\
\hline $43 a$ & $<2.5$ & 1,798 & $\cdots$ & 1,502 & 3.30 & $<15.6$ & -- & 1.51 & 4.77 & 0.029 & 1.97 \\
\hline $44 b$ & $<2.5$ & 1,118 & --- & 4,782 & 8.90 & $<15.6$ & $\ldots$ & 4.16 & 13.7 & 0.048 & 3.31 \\
\hline $45 a$ & $<8.5$ & 28,128 & -- & 53,302 & 81.4 & $<85.6$ & $-\cdots$ & 7.83 & 75.5 & 0.343 & 23.7 \\
\hline Average & $\mathrm{ND}$ & 24,795 & & 46,179 & 92.70 & ND & & 8.56 & 76.6 & 0.399 & 27.5 \\
\hline $\operatorname{Max}$ & $-\cdots$ & 72,428 & & 167,582 & 284.00 & --- & & 19.40 & 253.00 & 1.17 & 81.00 \\
\hline
\end{tabular}


Total Contamination: Metal Sample Analytical Results (Source: Hill and Hobart 2000, Table 7)

\begin{tabular}{|c|c|c|c|c|c|c|c|c|c|}
\hline \multirow[b]{2}{*}{ Sample } & \multirow{2}{*}{$\begin{array}{c}\text { Etched } \\
\text { Surface } \\
\text { Area }\left(\mathrm{cm}^{2}\right) \\
\end{array}$} & \multirow{2}{*}{$\begin{array}{c}\text { Etched } \\
\text { Area } \\
\text { Mass (g) } \\
\end{array}$} & \multirow[b]{2}{*}{$\mathrm{g} / \mathrm{cm}^{2}$} & \multicolumn{2}{|c|}{ Total Activity } & \multicolumn{2}{|c|}{ Total Alpha } & \multicolumn{2}{|c|}{ Total Beta-Gamma } \\
\hline & & & & $(\mathrm{nCi} / \mathrm{g})$ & $\begin{array}{c}(\mathrm{nCi} / \\
\left.100 \mathrm{~cm}^{2}\right) \\
\end{array}$ & $(\mathrm{nCi} / \mathrm{g})$ & $\begin{array}{c}(\mathrm{nCi} / \\
\left.100 \mathrm{~cm}^{2}\right)\end{array}$ & $(\mathrm{nCi} / \mathrm{g})$ & $\begin{array}{c}(\mathrm{nCi} / \\
\left.100 \mathrm{~cm}^{2}\right) \\
\end{array}$ \\
\hline $1-1 a$ & 5.30 & 5.39 & 1.02 & 72,838 & $7.41 \mathrm{E}+06$ & 366 & $3.72 E+04$ & 72,472 & $7.37 E+06$ \\
\hline $1-1 b$ & 8.69 & 8.83 & 1.02 & 23,674 & $2.41 \mathrm{E}+06$ & 96 & $9.74 \mathrm{E}+03$ & 23,578 & $2.40 \mathrm{E}+06$ \\
\hline $1-2 a$ & 6.52 & 6.62 & 1.02 & 56,359 & $5.72 E+06$ & 321 & $3.26 \mathrm{E}+04$ & 56,038 & $5.69 \mathrm{E}+06$ \\
\hline $1-2 b$ & 6.54 & 6.64 & 1.02 & 38,759 & $3.94 \mathrm{E}+06$ & 129 & $1.31 E+04$ & 38,630 & $3.92 E+06$ \\
\hline $3-1 a$ & 6.44 & 6.54 & 1.02 & 45,187 & $4.59 \mathrm{E}+06$ & 77 & $7.77 E+03$ & 45,110 & $4.58 E+06$ \\
\hline $3-1 b$ & 7.45 & 7.57 & 1.02 & 24,007 & $2.44 \mathrm{E}+06$ & 41 & $4.22 E+03$ & 23,965 & $2.44 \mathrm{E}+06$ \\
\hline $3-2 a$ & 9.97 & 10.12 & 1.02 & 41,931 & $4.26 \mathrm{E}+06$ & 80 & $8.08 \mathrm{E}+03$ & 41,851 & $4.25 E+06$ \\
\hline $3-2 b$ & 5.25 & 5.33 & 1.02 & 26,063 & $2.65 \mathrm{E}+06$ & 47 & $4.78 \mathrm{E}+03$ & 26,016 & $2.64 E+06$ \\
\hline 1A-1a & 27.90 & 57.65 & 2.07 & 8,579 & $1.77 \mathrm{E}+06$ & 8 & $1.60 \mathrm{E}+03$ & 8,571 & $1.77 E+06$ \\
\hline $1 A-1 b$ & 12.00 & 23 & 1.92 & 12,966 & $2.49 \mathrm{E}+06$ & 14 & $2.67 \mathrm{E}+03$ & 12,952 & $2.48 E+06$ \\
\hline 1A-1C & 5.52 & 9.81 & 1.78 & 3,538 & $6.29 \mathrm{E}+05$ & 15 & $2.59 E+03$ & 3,524 & $6.26 E+05$ \\
\hline $1 A-1 d$ & 12.00 & 22.21 & 1.85 & 17,201 & $3.18 \mathrm{E}+06$ & 22 & $4.06 \mathrm{E}+03$ & 17,180 & $3.18 E+06$ \\
\hline $1 \mathrm{~A}-1 \mathrm{e}$ & 11.19 & 19.29 & 1.72 & 6,268 & $1.08 \mathrm{E}+06$ & 5 & $8.11 E+02$ & 6,263 & $1.08 \mathrm{E}+06$ \\
\hline $1 A-1 f$ & 9.72 & 18.65 & 1.92 & 12,592 & $2.42 E+06$ & 6 & $1.08 E+03$ & 12,586 & $2.41 E+06$ \\
\hline $1 \mathrm{~A}-1 \mathrm{~g}$ & 14.57 & 29.46 & 2.02 & 29,981 & $6.06 \mathrm{E}+06$ & 16 & $3.31 E+03$ & 29,965 & $6.06 \mathrm{E}+06$ \\
\hline 1A-1h & 4.70 & 8.9 & 1.89 & 3,214 & $6.09 \mathrm{E}+05$ & 1 & $2.70 \mathrm{E}+02$ & 3,213 & $6.08 E+05$ \\
\hline $1 A-2$ & 21.80 & 25.26 & 1.16 & 4,424 & $5.13 E+05$ & 3 & $3.44 \mathrm{E}+02$ & 4,421 & $5.12 E+05$ \\
\hline $1 \mathrm{~A}-3$ & 42.90 & 54.83 & 1.28 & 1,152 & $1.47 \mathrm{E}+05$ & 1 & $6.91 E+01$ & 1,151 & $1.47 E+05$ \\
\hline $1 \mathrm{~A}-5$ & 44.50 & 53.86 & 1.21 & 1,254 & $1.52 \mathrm{E}+05$ & 3 & $3.99 E+02$ & 1,251 & $1.51 E+05$ \\
\hline $1 \mathrm{~A}-7$ & 31.80 & 39.41 & 1.24 & 4,749 & $5.89 \mathrm{E}+05$ & 32 & $3.96 \mathrm{E}+03$ & 4,717 & $5.85 E+05$ \\
\hline $1 \mathrm{~A}-8$ & 24.80 & 19.09 & 0.77 & 4,173 & $3.21 E+05$ & 4 & $2.82 E+02$ & 4,169 & $3.21 E+05$ \\
\hline $1 \mathrm{~A}-9$ & 33.20 & 81.11 & 2.44 & 3,241 & $7.92 E+05$ & 4 & $1.05 E+03$ & 3,237 & $7.91 E+05$ \\
\hline $1 \mathrm{~A}-10$ & 22.50 & 39.78 & 1.77 & 2,032 & $3.59 \mathrm{E}+05$ & 2 & $4.39 E+02$ & 2,030 & $3.59 E+05$ \\
\hline $1 \mathrm{~A}-11$ & 26.00 & 50.21 & 1.93 & 988 & $1.91 E+05$ & 1 & $1.65 E+02$ & 987 & $1.91 E+05$ \\
\hline 1A-12 & 18.20 & 43.4 & 2.38 & 8,282 & $1.98 \mathrm{E}+06$ & 7 & $1.78 \mathrm{E}+03$ & 8,275 & $1.97 \mathrm{E}+06$ \\
\hline $1 \mathrm{~A}-14$ & 58.90 & 106.7 & 1.81 & 2,580 & $4.67 \mathrm{E}+05$ & 7 & $1.32 E+03$ & 2,572 & $4.66 \mathrm{E}+05$ \\
\hline 2B-1a & 12.00 & 60.86 & 5.07 & 614 & $3.11 E+05$ & 3 & $1.58 \mathrm{E}+03$ & 611 & $3.10 E+05$ \\
\hline 2B-1b & 16.49 & 88.41 & 5.36 & 532 & $2.85 \mathrm{E}+05$ & 2 & $8.83 E+02$ & 530 & $2.84 E+05$ \\
\hline Average & $\mathrm{NA}$ & NA & 1.78 & 16,328 & $2.06 \mathrm{E}+06$ & 47 & $5.22 \mathrm{E}+03$ & 16,281 & $2.06 \mathrm{E}+06$ \\
\hline Max & NA & $\mathrm{NA}$ & 5.36 & 72,838 & $7.41 \mathrm{E}+06$ & 366 & $3.72 E+04$ & 72,472 & $7.37 E+06$ \\
\hline
\end{tabular}

Removable Contamination: Smear Analytical Results (Source: Hill and Hobart 2000, Table 9)

\begin{tabular}{|c|c|c|c|c|c|c|}
\hline \multirow[b]{2}{*}{ Sample } & \multicolumn{2}{|c|}{ Total Activity } & \multicolumn{2}{|c|}{ Total Alpha } & \multicolumn{2}{|c|}{ Total Beta-Gamma } \\
\hline & $\begin{array}{c}\text { (nCi/ } \\
\text { smear) }\end{array}$ & $\begin{array}{c}(\mathrm{nCi} / \\
\left.100 \mathrm{~cm}^{2}\right)\end{array}$ & $\begin{array}{c}\text { (nCi/ } \\
\text { smear) }\end{array}$ & $\begin{array}{c}(\mathrm{nCi} / \\
\left.100 \mathrm{~cm}^{2}\right)\end{array}$ & $\begin{array}{c}\text { (nCi/ } \\
\text { smear) }\end{array}$ & $\begin{array}{c}(\mathrm{nCi} / \\
\left.100 \mathrm{~cm}^{2}\right)\end{array}$ \\
\hline $39 b$ & 240,471 & $2.40 E+05$ & 177 & $1.77 \mathrm{E}+02$ & 240,294 & $2.40 E+05$ \\
\hline $40 \mathrm{~b}$ & 10,135 & $1.01 E+04$ & 10 & $1.04 \mathrm{E}+01$ & 10,125 & $1.01 E+04$ \\
\hline $42 a$ & 85,609 & $8.56 \mathrm{E}+04$ & 355 & $3.55 \mathrm{E}+02$ & 85,254 & $8.53 E+04$ \\
\hline $43 a$ & 3,312 & $3.31 E+03$ & 8 & $8.28 \mathrm{E}+00$ & 3,303 & $3.30 E+03$ \\
\hline $44 b$ & 5,930 & $5.93 E+03$ & 21 & $2.12 E+01$ & 5,909 & $5.91 E+03$ \\
\hline $45 a$ & 81,619 & $8.16 \mathrm{E}+04$ & 107 & $1.07 E+02$ & 81,511 & $8.15 E+04$ \\
\hline Average & 71,179 & $7.12 E+04$ & 113 & $1.13 E+02$ & 71,066 & $7.11 E+04$ \\
\hline Max & 240,471 & $2.40 \mathrm{E}+05$ & 355 & $3.55 \mathrm{E}+02$ & 240,294 & $2.40 E+05$ \\
\hline
\end{tabular}

$\begin{array}{cccc}\text { Average } & 28.97 & 46.15 & \\ \text { Max } & 30.80 & 104.88 & 28.94 \\ & & & 30.67\end{array}$




\section{DISTRIBUTION}

\section{Washington Closure Hanford}

R. J. Reeder

L4-30

J. C. Cooper

L $1-10$

S. H. Jenkins

L1-10

R. F. Patch

L7-11

G. A. Simiele

H4-25

Document Control

H4-11

DOE-RL Public Reading Room

$\mathrm{H} 2-53$

Hanford Technical Library

P8-55 